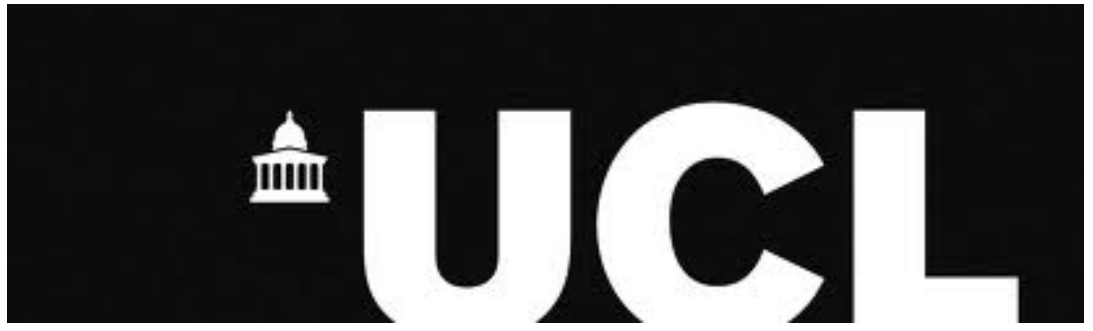

\title{
THE LONG-TERM PROGNOSIS OF EPILEPSY
}

\author{
AIDAN NELIGAN \\ UCL Institute of Neurology \\ Queen Square \\ London WC1N 3BG
}

A thesis submitted to University College London for the degree of Doctor of Philosophy 2011. 


\section{Declaration}

I, Aidan Neligan, confirm that the work presented in this thesis is my own. Dr Gail Bell calculated the standardised mortality ratios (SMRs) and the standardised incidence rates (SIRs) for the NGPSE study and reproduced the Kaplan-Meier graphs in Stata after I produced the original graphs in SPSS. Where information has been derived from other sources, I confirm that this has been indicated in the thesis. 


\section{Abstract}

The five studies presented in this thesis consider different aspects of the long-term prognosis of epilepsy and febrile seizures. The studies were:

1). A systemic review to examine a) how the risk of premature mortality in an individual with epilepsy changes over time and b) whether population mortality rates due to epilepsy have changed over time. (Study 1)

2). An extension of the National General Practice Study of Epilepsy (NGPSE), a prospective community-based incident cohort study to examine a) long-term seizure prognosis and mortality in people with epilepsy and b) seizure prognosis in children with febrile seizures (Study 2)

3). Two retrospective hospital-based cohort studies examining prognosis of chronic epilepsy with regard to a) frequency of different seizure patterns (Study 3) and mediumterm seizure outcome following anti-epileptic drug changes (Study 4).

4). A systematic review examining the impact of aetiology and others factors on outcome in status epilepticus (Study 5).

Amongst the findings were:

1): There is no conclusive evidence that either the overall standardised mortality ratio (SMR) or the mortality rate of people with epilepsy has changed significantly over time. The SMR is highest soon after diagnosis and subsequently decreases with a possible late increase after 10 years. In the NGPSE cohort the SMR remains significantly elevated after 20 years despite over $80 \%$ currently being in terminal remission.

2): $6.7 \%(95 \%$ CI $4,11 \%)$ of children with febrile seizures developed epilepsy after 20 years.

3): Approximately one-third of people with chronic epilepsy have a history of at least one significant period of seizure freedom (two or more years) while a comparable number with apparent drug-resistant epilepsy attain at least one year of seizure freedom after medication change, although approximately half subsequently relapse. 
4). Aetiology and, to a lesser extent, age are the primary determinants of prognosis in status epilepticus. 


\section{TABLE OF CONTENTS}

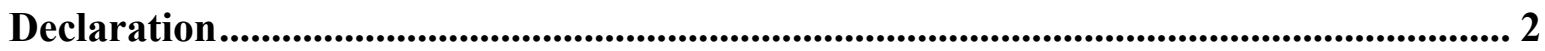

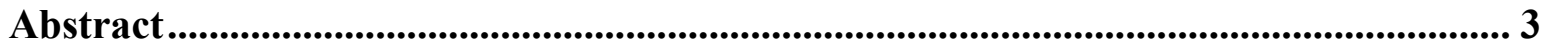

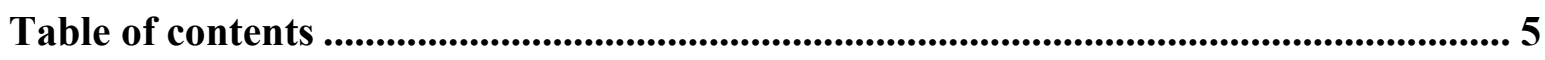

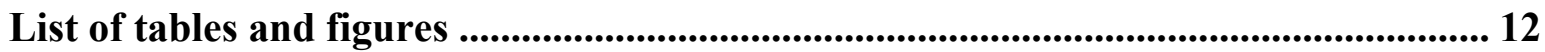

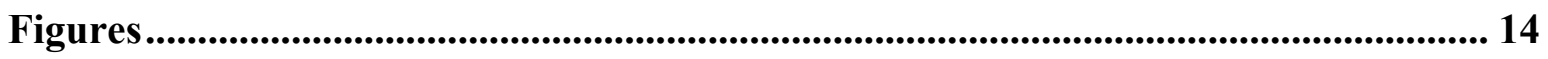

Acknowledgements................................................................................................................. 16

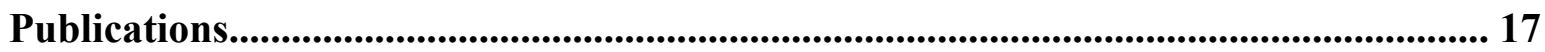

Chapter 1: Introduction and literature review ........................................................ 19

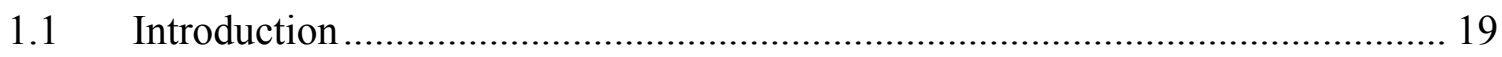

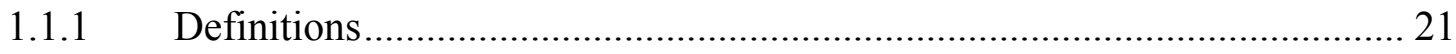

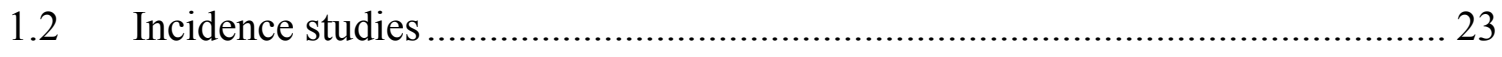

1.2.1 Incidence of epilepsy in more developed countries ............................... 24

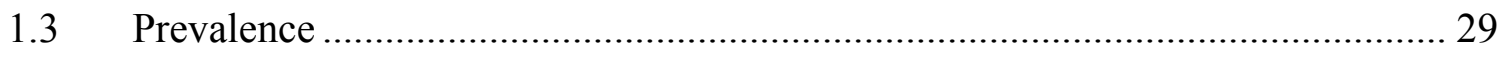

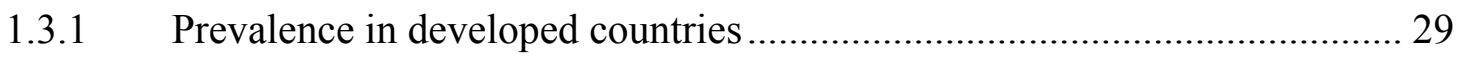

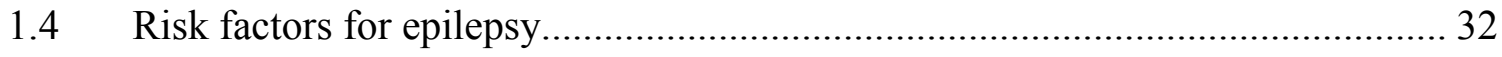

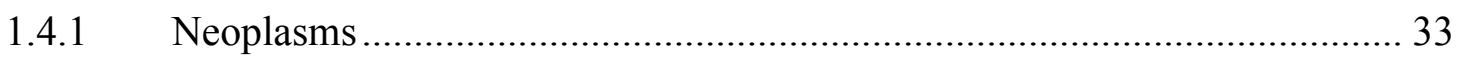

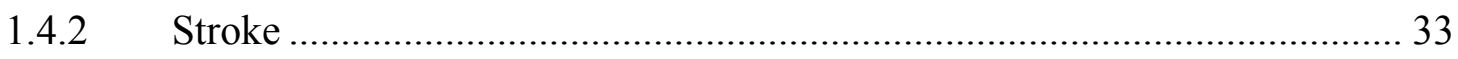

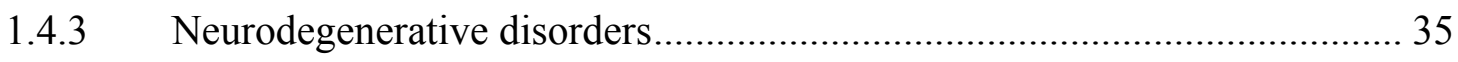

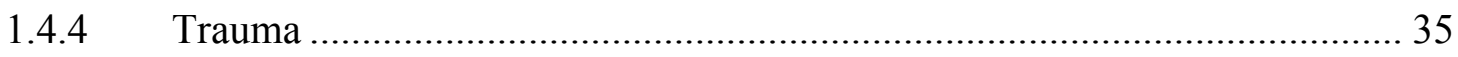

1.4.5 Seizures and bacterial meningitis/encephalitis..................................... 37

1.4.6 Seizures and other CNS infections and infestations ............................. 38

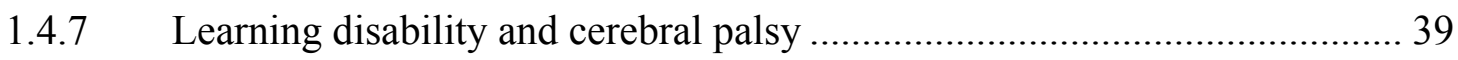


1.5 Prognosis

1.5.1 Recurrence after a single seizure ........................................................ 41

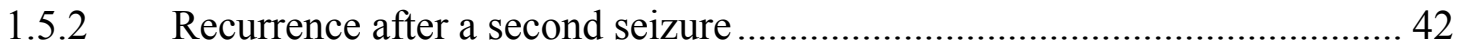

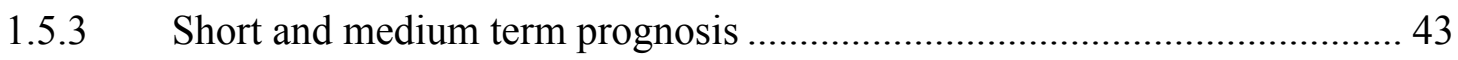

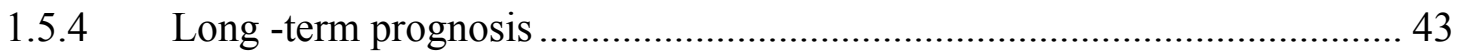

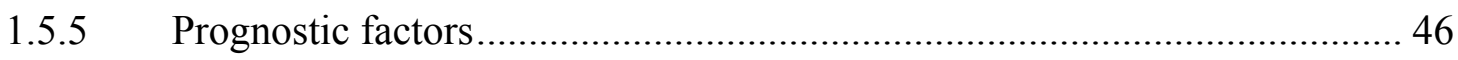

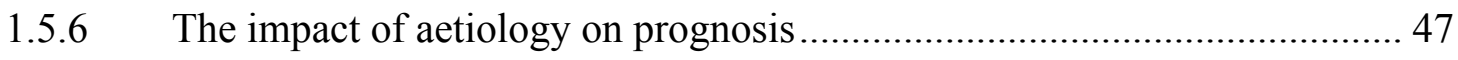

1.5.7 The impact of anti-epileptic drugs on prognosis.................................... 48

1.5.8 Prognosis following AED withdrawal ............................................. 50

1.5.9 Patterns of relapse and remission in epilepsy ....................................... 52

1.5.10 The prognosis for those with intractable epilepsy.................................. 53

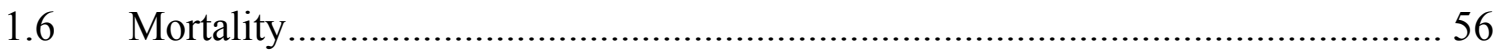

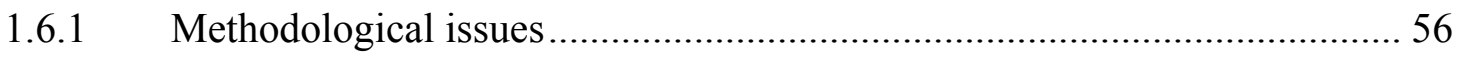

1.6.2 Mortality following a newly diagnosed seizure ................................... 58

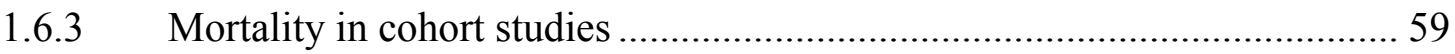

1.6.4 Temporal trends in mortality in people with epilepsy ............................. 59

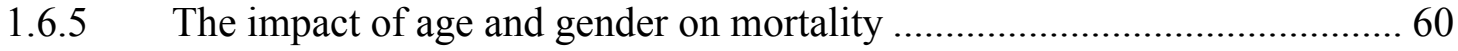

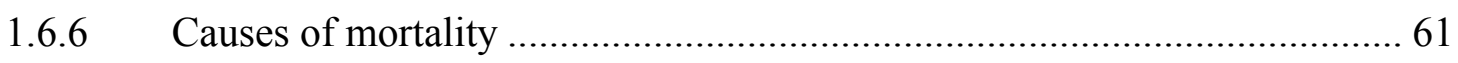

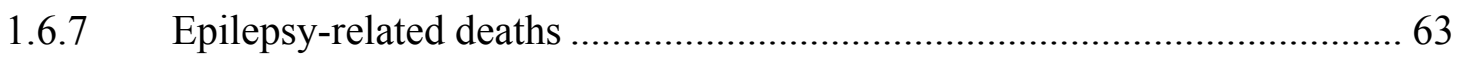

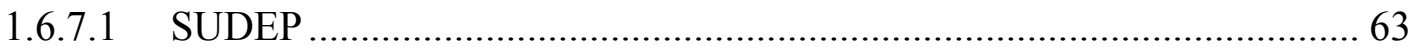

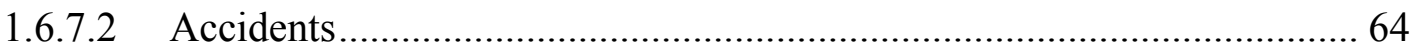

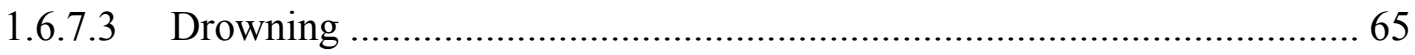

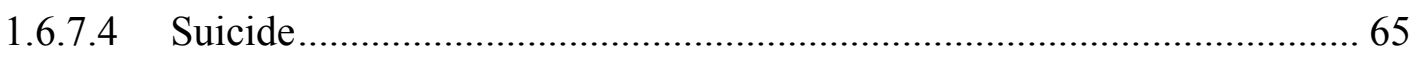

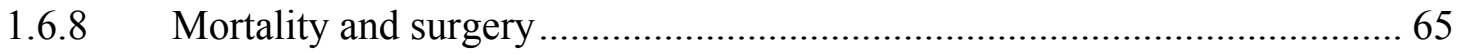

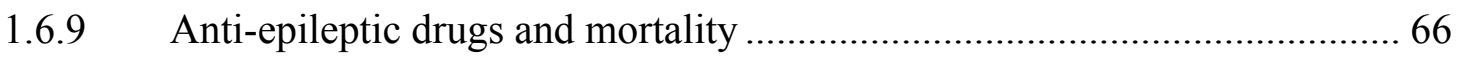




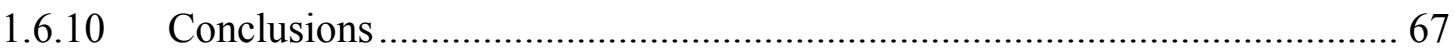

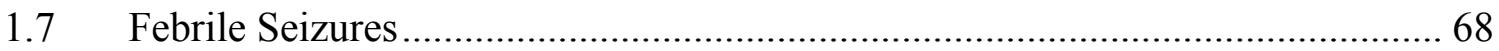

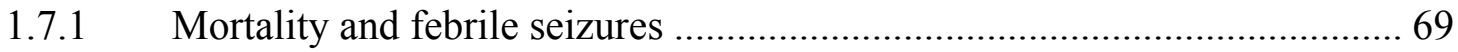

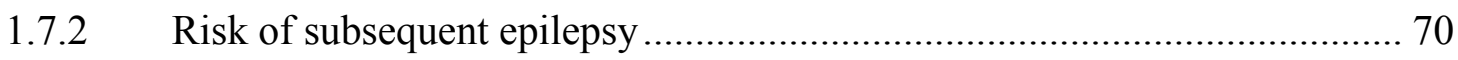

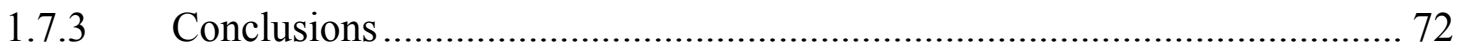

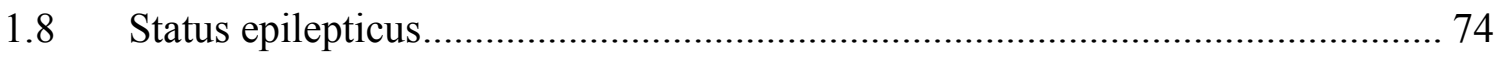

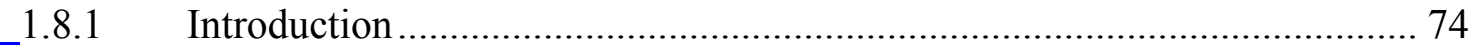

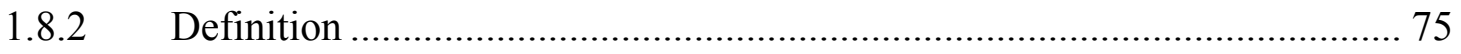

1.8.3 The epidemiology of status epilepticus....................................................... 77

Chapter 2: Aims ...................................................................................................................... 81

2.1 Aims of the Systematic Review of the Temporal Trends in Mortality in People

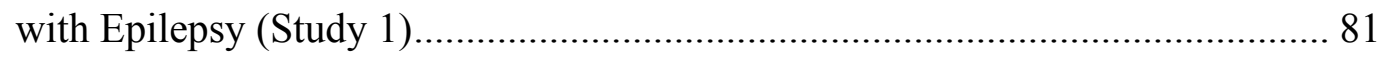

2.2 Aims of this extension to the National General Practice Study of Epilepsy and

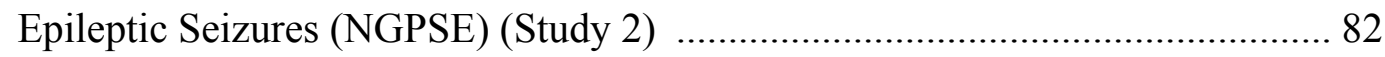

2.3 Aims of the Seizure Patterns in Chronic Epilepsy Study (Study 3).................... 83

2.4 Aims of the Impact of Medication Change in people with Chronic Epilepsy Study

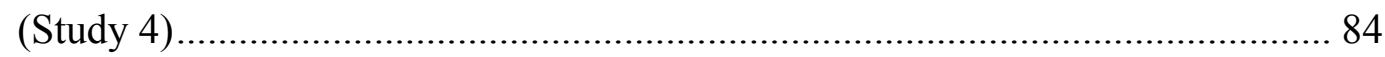

2.5 Aims of the Systematic Review of the Prognosis of Status Epilepticus (Study 5)..

\section{Chapter 3: The Systematic Review of the Temporal Trends in Mortality in people} with epilepsy (Study 1).......................................................................................... 86

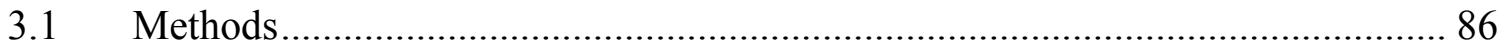

3.2 Changes in SMR in people with epilepsy over the course of the condition ........ 87 


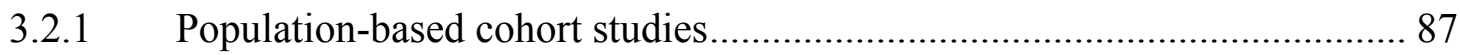

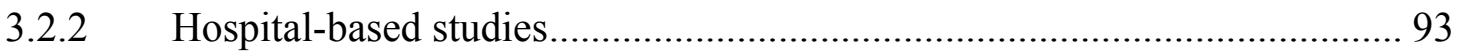

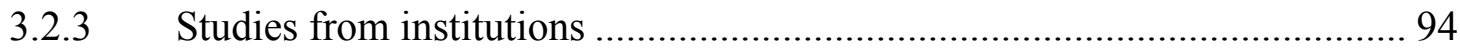

3.3 Population-based mortality trends in people with epilepsy ............................. 96

\section{Chapter 4: Extension of the National General Practice Study of Epilepsy (NGPSE)}

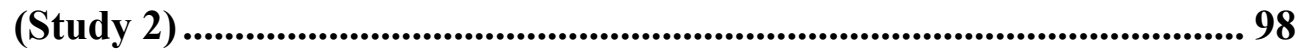

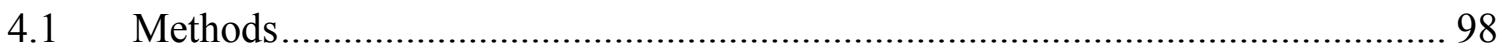

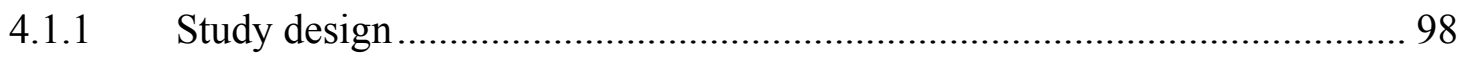

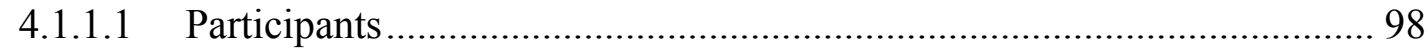

4.1.1.2 Classification............................................................................. 98

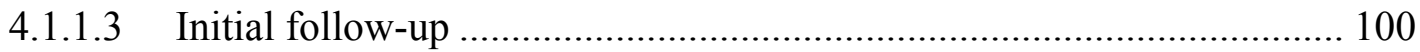

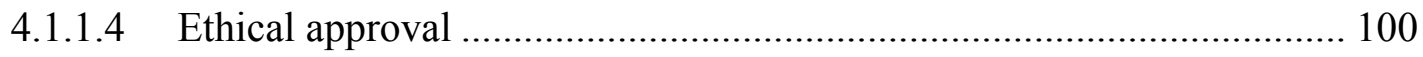

4.1.2 Data protection and the implications for the NGPSE ........................... 101

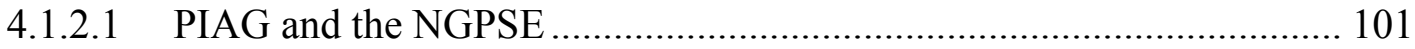

4.1.2.2 The Office of National Statistics (ONS) and the NGPSE.................... 104

4.1.2.3 Research \& Development Departments ........................................... 104

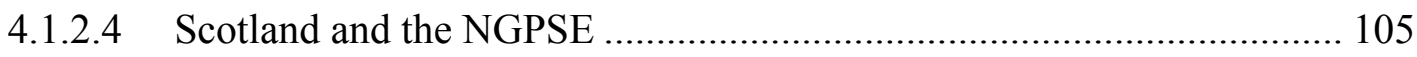

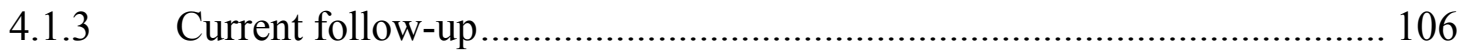

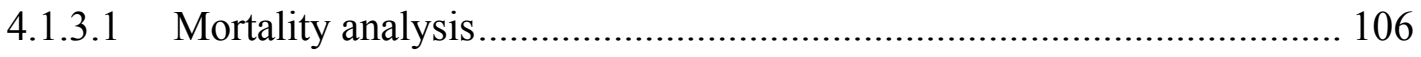

4.1.3.2 Mortality statistical analysis: statistical analysis............................. 107

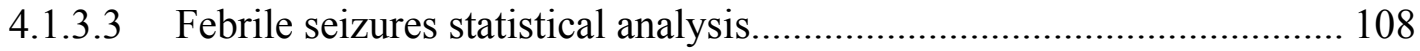

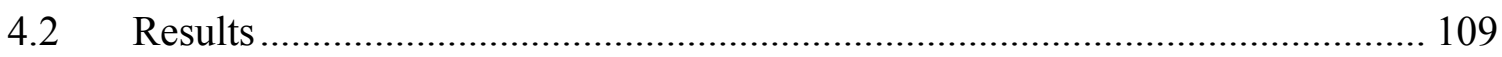

4.2.1 The long-term risk of mortality in people with epilepsy.......................... 109

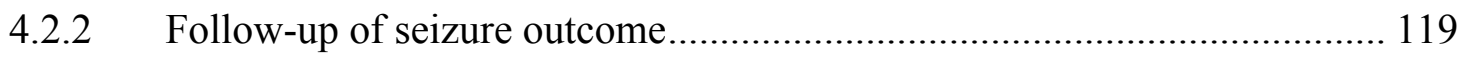


4.2.3 The long-term prognosis of people with febrile seizures.......................... 121

4.2.4 The long-term prognosis of people with epilepsy.................................. 125

4.2.4.1 Survival analysis of time to first seizure and Cox regression model ..... 129

4.2.4.2 The impact of aetiology on prognosis in definite epilepsy .................. 133

Chapter 5: The Study of Seizure Patterns in people with chronic epilepsy (Study 3)

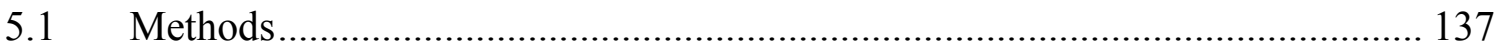

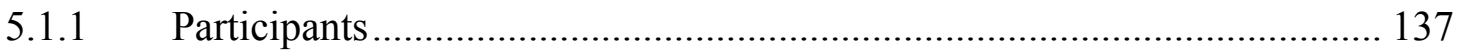

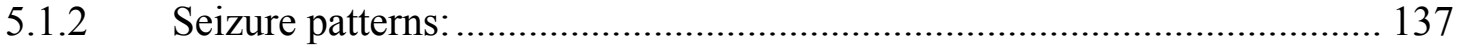

5.1.3 Seizure and syndrome classification: ................................................ 138

5.1.4 Ethical approval: .................................................................... 139

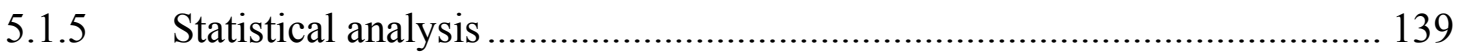

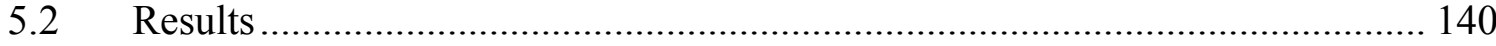

Chapter 6: The Study of the Impact of Medication change in People with Chronic Epilepsy (Study 4) ........................................................................................... 145

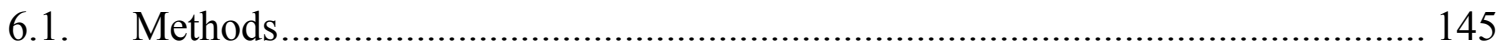

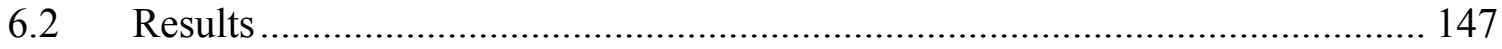

Chapter 7: The Systematic Review of the Prognosis of Convulsive Status

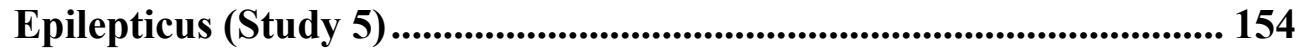

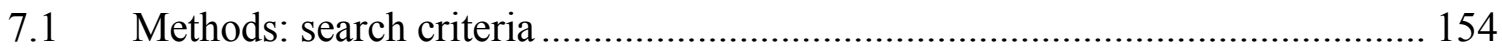

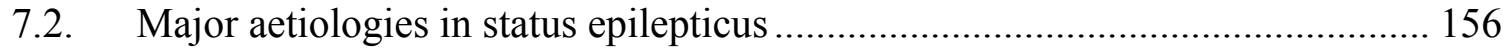

7.3 Frequency and prognosis of different aetiologies ..................................... 161 


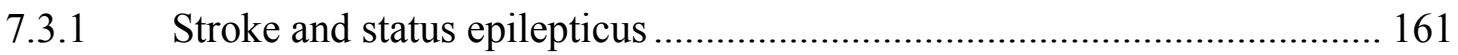

7.3.2 Alcohol, substance abuse and drug-induced status epilepticus.................. 164

7.3.3 Status epilepticus and antiepileptic drug reduction or withdrawal or low antiepileptic drug levels ........................................................... 165

7.3.4 Severe acute cerebral anoxia/hypoxia and status epilepticus................... 167

7.3.5 CNS infections, encephalitis and status epilepticus.............................. 168

7.3.6 Cerebral Tumour and status epilepticus ............................................... 171

7.3.7 Trauma and status epilepticus ......................................................... 171

7.3.8 Metabolic disorders and status epilepticus...................................... 172

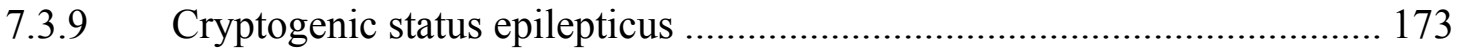

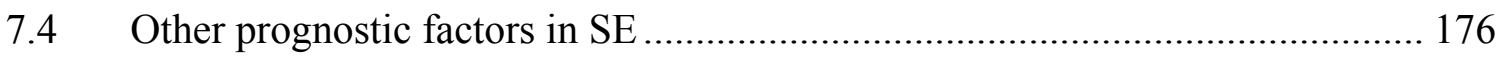

7.4.1. The duration of status epilepticus and its relation to outcome.................. 176

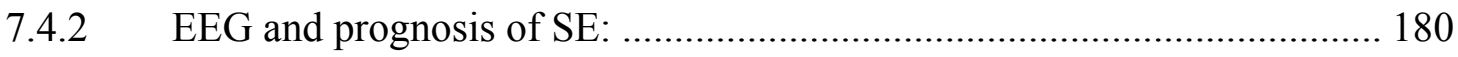

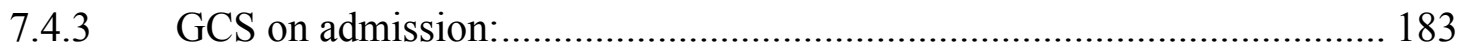

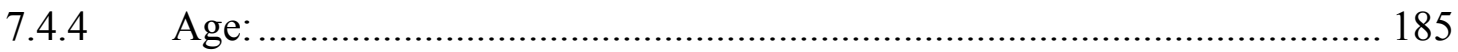

7.4.4.1 Status epilepticus in children: ................................................ 185

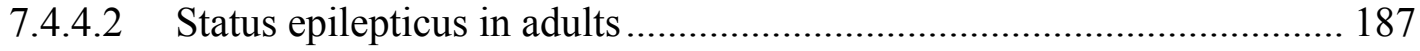

7.5 Outcome measures in status epilepticus.................................................. 191

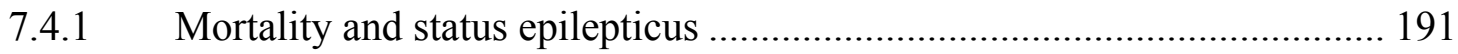

7.4.2 Morbidity - neurological deficit and status epilepticus .......................... 192

7.4.3 Morbidity - neuro-cognitive sequelae of status epilepticus..................... 194

7.4.4 Morbidity - the risk of epilepsy after status epilepticus ........................... 195 
Chapter 8: Discussion ........................................................................................................ 197

8.1 Epilepsy and mortality (Studies 1\&2) ........................................................ 197

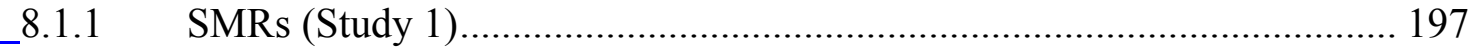

8.1.2 The long-term risk of premature mortality (Studies 1\&2) ......................... 199

8.1.3 Temporal trends in mortality (Study 1).................................................. 205

8.1.4 Changing mortality rates over time (Study 1) ………………………...... 207

8.2 The long-term prognosis of people with epilepsy (Study 2)............................ 209

8.3 The long-term prognosis of people with febrile seizures (Study 2)….............. 215

8.4 The prognosis of chronic epilepsy (Studies 3 \& 4) …………........................ 219

8.4.1 Seizure patterns in chronic epilepsy (Study 3).......................................... 219

8.4.2 The impact of medication change in chronic epilepsy (Study 4).............. 222

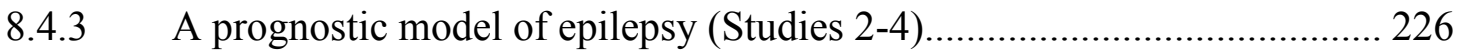

8.5 The prognosis of status epilepticus (Study 5) ………………....................... 230

8.6 Problems facing epidemiological research in the UK: lessons from the NGPSE....

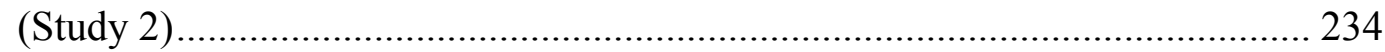

Chapter 9: Conclusions and future directions ................................................................... 240

Reference List ......................................................................................................................... 242

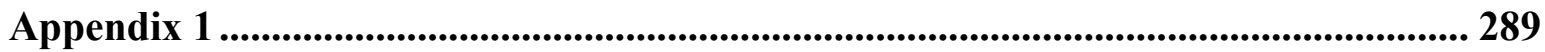

Appendix 2:........................................................................................................................... 292 


\section{List of tables and figures}

\section{Tables}

Table 1: Incidence of first epileptic seizure (per 100,000 people) ................................. 24

Table 2: Prospective population-based incidence studies .......................................... 26

Table 3: Incidence studies in resource poor countries ................................................. 27

Table 4: Incidences of epileptic syndromes (per 100,000 person-years)......................... 28

Table 5: Selected prevalence studies from resource-poor countries ................................. 31

Table 6: Long-Term Follow-up Studies in Epilepsy ................................................ 45

Table 7: Population-based studies of status epilepticus (Study 5) ................................ 80

Table 8: Population studies of mortality in people with epilepsy with SMRs (with 95\% CIs)

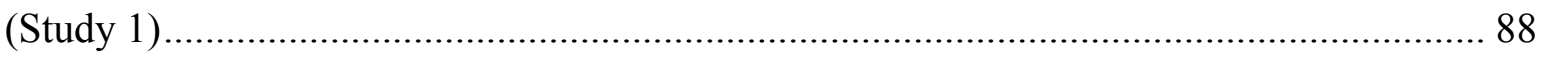

Table 9: Population studies of mortality in people with epilepsy with SMRs (with 95\% CIs)

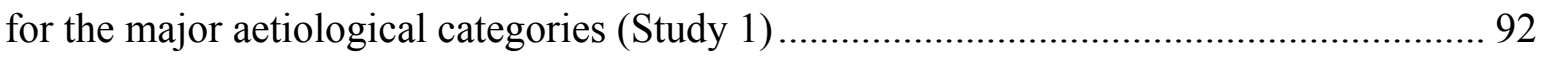

Table 10: All-cause mortality for Whole Cohort and Specific Subgroups (Study 2) ....... 110

Table 11: All-cause mortality in people with definite epilepsy by aetiology (Study 2) .... 112

Table 12: Cause-specific SMRs in different subgroups (Study 2)................................ 116

Table 13: Selected causes of death (with SMRs and 95\% Confidence Intervals) for people

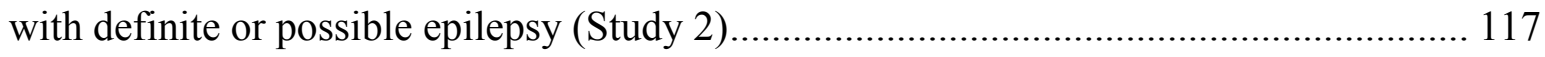

Table 14 Basic follow-up data from the NGPSE cohort 2009-10 (Study 2) ................... 120

Table 15: Outcome of people with febrile seizures who developed epilepsy (Study 2)... 124

Table 16: Seizure outcomes in those with definite and possible epilepsy (Study 2) ........ 127

Table 17: Seizure outcomes in people in whom the index seizure was the first seizure

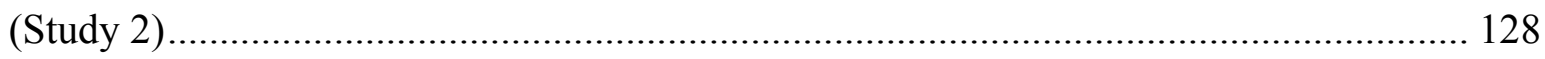


Table 18: Proportion of people with definite and possible epilepsy who attain at least five year seizure freedom in the first ten years of follow-up (Study 2)

Table 19: Seizure outcome in those classified as having definite epilepsy by aetiology (Study 2)

Table 20: Basic demographics and epilepsy features (Study 3)

Table 21: Frequency of the predominant seizure type and epileptic syndromes in seizure

patterns (Study 3)

Table 22: Seizure frequency in the previous 12 months in people with continuous and intermittent seizures patterns (Study 3)

Table 23: List of drug changes in people with extended follow-up (Study 4)..... 148

Table 24: Seizure outcome following individual medication changes in people with prolonged follow-up.(Study 4). 149

Table 25: Seizure outcomes in 2004 and 2009 (Study 4) 150

Table 26: Major Aetiologies of Status Epilepticus (Study 5) 158

Table 27: Studies of Status Epilepticus in the developing world (Study 5)..... 160

Table 28: Duration and Status Epilepticus (Study 5) 178

Table 29: The EEG and the prognosis of Status Epilepticus (Study 5) 182

Table 30: Level of consciousness and Status Epilepticus (Study 5) 184

Table 31: Age and Status Epilepticus (Study 5) 189

Table 32: Approximate frequency and mortality of status epilepticus in different aetiologies in representative Western populations (Study 5) 231 


\section{Figures}

Figure 1: All cause mortality by age-group during follow-up in the combined group with definite and possible epilepsy, people with definite epilepsy, and the subgroup with idiopathic epilepsy (Study 2)

Figure 2: Mortality by years of follow-up from index seizure in the combined group with definite and possible epilepsy, people with definite epilepsy, and the subgroup with idiopathic epilepsy (Study 2)

Figure 3: Cumulative risk of febrile seizure recurrence in relation to the index seizure (Study 2)

Figure 4: Time to develop epilepsy in people with febrile seizures (Study 2)

Figure 5: Kaplan-Meier analysis of probability of remaining seizure free after index seizure for those with definite, possible and febrile seizures (Study 2)

Figure 6: Kaplan-Meier analysis of probability of remaining seizure free after index seizure in those with definite epilepsy stratified by aetiological grouping (Study 2) 135

Figure 7: Kaplan-Meier analysis of the probability of remaining seizure free (Study 4).. 152 
Figure 8: Kaplan-Meier analysis of the probability of remaining seizure free comparing the

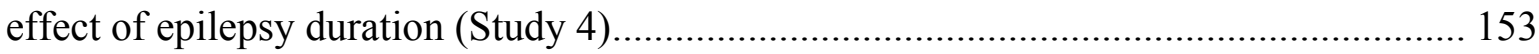

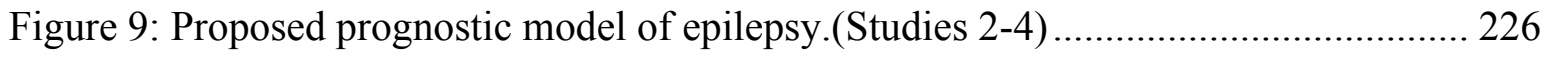




\section{Acknowledgements}

Firstly, I wish to sincerely thank Professors Simon Shorvon and Ley Sander who I was very fortunate to have as my supervisors. It is because of their different but equally inspirational guidance that this thesis has been completed. Thank you also for your forbearance towards my judicious "adherence" to agreed deadlines.

I owe an enormous debt of gratitude to my friend and colleague Dr Gail Bell without whose help this thesis would never have been completed in the current time-scale. Thank you for your patience with my Hiberno-English, your brilliant editing skills, your help with and many discussions on statistics but most especially for your friendship over the past four years.

I am grateful to the other members of the NGPSE study, in particular Dr Christina Gravassi for her help with the data entry; Dr David Goodridge for his help in contacting GP practices and insightful comments and to Dr Tony Johnson for his statistical expertise and input.

I wish to sincerely acknowledge all the local managers of the PCRN who help promote and highlight the NGPSE to the relevant GP practices throughout England. I would particularly like to thank Drs Jenny Baverstock, Claire Pentecost and Miss Becky Dilley among others for their help.

I wish to thank all the GPs who completed the questionnaires (many of whom I had the pleasure of talking to) and the many GP practice managers that I talked to and whom help facilitate the completion of the questionnaires. Thank you to all patients who kindly agreed to take part in the clinical studies.

To all my friends at the Institute particularly Juliet, Jennifer, Patrick and Lisa among others, thank you for putting up with my incessant moaning whilst trying to write up and keeping me saneish.

To my parents Michael and Rose, thank you for your continued love and support and for never questioning the wisdom of my coming to London.

Finally I wish to dedicate this thesis to my daughter Alice and sincerely thank and acknowledge my beautiful wife Oönagh, without whom none of this would have been possible. 


\section{Publications}

Neligan A, Bell GS, Giavasi C et al (2012). The long-term risk of developing epilepsy after febrile seizures: a prospective cohort study. Neurology (IN PRESS)

Neligan A, Bell GS, Sander JW et al (2011). How refractory is refractory epilepsy?

Patterns of relapse and remission in people with refractory epilepsy. Epilepsy Res 96: 225230

Neligan A and Sander JW (2011). Premature mortality in epilepsy: is it preventable? Expert Rev. Theraper 11: 767-770.

Neligan A, Bell GS, Johnson AL et al (2011). The long-term risk of premature mortality in people with epilepsy. Brain 134: 388-395.

Neligan A, Bell GS, Shorvon SD et al (2010). Temporal trends in the mortality of people with epilepsy: A review. Epilepsia 51: 2241-6.

Neligan A and Shorvon SD (2011). Prognostic factors, morbidity and mortality in tonicclonic status epilepticus: a review. Epilepsy Res 93: 1-10.

Neligan A and Shorvon SD (2010). Frequency and prognosis of convulsive status epilepticus of different causes: a systematic review. Arch Neurol 67: 931-940. 


\section{Book chapters}

Neligan A and Sander JW (2011). The Incidence and Prevalence of Epilepsy.

Sander JW, Rugg-Gunn FJ, Smalls JE (Eds) Epilepsy 2011: Epilepsy 2011: From Science to Society, a practical guide to epilepsy. ILAE, pp15-22.

Neligan A and Sander JW (2011). The Prognosis of Epilepsy. Sander JW, Rugg-Gunn FJ, Smalls JE (Eds) Epilepsy 2011: From Science to Society, a practical guide to epilepsy. ILAE, pp357-364.

Neligan A and Bell GS (2011). The Mortality of Epilepsy. Sander JW, Rugg-Gunn FJ, Smalls JE (Eds) Epilepsy 2011: From Science to Society, a practical guide to epilepsy. ILAE, pp365-372.

Neligan A and Shorvon SD (2008). Refractory versus non-refractory status epilepticus: frequency, differentiating clinical features, and outcome. Kahane,P., Berg,A.T., Loscher,W., Perucca,E. (Eds.) Drug-Resistant Epilepsies. John Libbey EUROTEXT, pp. $29-46$. 


\section{Chapter 1: Introduction and literature review}

\subsection{Introduction}

Epilepsy is the most common neurological condition affecting people of all ages, races and social classes. Worldwide it is estimated that over 50 million people have epilepsy of whom up to $75 \%$ live in resource-poor countries with little or no access to medical services and treatment (Meinardi et al., 2001; Ngugi et al., 2010).

Epidemiology is the study of health determinants, and the distribution and natural history of disease in human populations. Epidemiological studies can be descriptive, analytical or experimental. Descriptive epidemiology is concerned with the distribution and vital statistics (incidence and prevalence) of a condition in a population, without regard to causation or other hypothesis and usually involves observational studies. Analytical epidemiology tests hypotheses, for example examining whether a certain exposure is a risk factor for a particular disease. This is normally examined by means of cohort and case control studies. Experimental epidemiology consists of studies in which the investigator is able to control relevant risk factors. Epidemiological studies in epilepsy are mostly descriptive and analytical with few experimental epidemiological studies conducted (Jallon, 2002; Sander, 2003).

Until the 1960s studies in epilepsy were carried out in tertiary referral centres; this favoured the belief that epilepsy was a chronic, progressive, incurable condition with little chance of remission (Sander ,1993; Berg and Shinnar, 1994). This view was endorsed in the $19^{\text {th }}$ century - "The spontaneous cessation of the disease is an event too 
rare to be reasonably anticipated in any given case" (Gowers, 1881) and reiterated years later, with epilepsy portrayed as "a chronic condition prone to relapse" (Rodin, 1968).

Since then many epidemiological studies have been published from both the resourcepoor and the developed world. Methodological differences, lack of standardised classification, problems with case ascertainment and diagnostic accuracy resulted in disparity in study findings as well as reflecting the heterogeneous nature of epilepsy.

Diagnostic accuracy is a particular problem in epilepsy as seizures are a symptom of diverse underlying cerebral aetiologies and may not have any permanent physical manifestations (Sander and Shorvon, 1987). This is further complicated by the lack of validated criteria for the diagnosis of an epileptic seizure. As a result a diagnosis of a seizure disorder is dependent on the chance recording of an event, the availability of a detailed eye-witness account (which may itself be misleading; Rugg-Gunn et al., 2001) and the expertise and experience of the diagnosing specialist, with opinions differing in ambiguous cases (Stroink et al., 2004; van Donselaar et al., 1989). Consequently a definitive diagnosis of epilepsy is often only made after an extended period of follow-up (Hauser and Kurland, 1975; Sander et al., 1990a). Moreover 20-30\% of those attending tertiary referral centres with refractory epilepsy do not in fact have epilepsy (Lesser, 1996; Smith et al., 1999) with the most common differential diagnoses being dissociative seizures and syncope. As expected, neurologists are better at diagnosing epilepsy than non-specialists (mistake rate 5.6\% versus 18.9\%) (Leach et al., 2005) but at least $5 \%$ of diagnoses may be wrong.

Many people with epilepsy may not come to medical attention, either through ignorance or lack of awareness of the symptoms. This is particularly true of absence and minor 
complex partial seizures which may only be recognised in retrospect following presentation with a generalised seizure (Hauser et al., 1990). Indeed, one general practice study found that only $20 \%$ of patients with seizures suspected the diagnosis prior to medical consultation (Hopkins and Scambler, 1977). In a community study in Warsaw where identification of cases was supplemented with a door to door survey, it was found that one quarter of people with seizures had not sought medical attention (Zielinski, 1974a).

People with epilepsy may also try to conceal it for fear of stigmatisation. This may be a particular problem in certain communities as seen in a door to door survey in Sicily where the low prevalence rate was interpreted as being the result of concealment (Rocca et al., 2001). In Australia, 10\% of those with a confirmed diagnosis of epilepsy (defined as having three seizures diagnosed by a doctor) denied having seizures (Beran et al., 1985).

Thus epidemiological studies of epilepsy should ideally be community-based, prospective (to identify risk factors) and independent of existing medical records (or supplemented by use of door to door surveys, questionnaires or interview) to allow as complete as possible case ascertainment (Sander and Shorvon, 1987).

\subsubsection{Definitions}

To allow meaningful comparisons of incidence and prevalence rates from different countries, the same definition of epilepsy and a standardised classification of seizures need to be applied across all studies. The International League Against Epilepsy (ILAE) (Commission on Epidemiology and Prognosis ILAE, 1993) provides guidelines for epidemiological studies on epilepsy. Epilepsy is defined as a condition characterised by recurrent seizures (two or more) which are unprovoked by any identifiable cause. 
Febrile seizures and neonatal seizures (within the first four weeks of life) are excluded. A single or isolated seizure is defined as one seizure or as multiple seizures occurring within a 24 hour period. A prevalent case of active epilepsy is defined as a person with known epilepsy who has had at least one unprovoked seizure in the previous five years irrespective of treatment status while a prevalent case of epilepsy in remission is defined as a person with known epilepsy who has had no seizures in five or more years regardless of treatment. Epidemiological studies in epilepsy are typically divided into studies from resource-rich and -poor countries because of differences in aetiologies and medical services; this division is somewhat arbitrary. 


\subsection{Incidence studies}

The incidence of epilepsy is defined as the number of new cases of epilepsy or seizures in a well-defined population during a specified time period (usually one year) and is normally expressed as the number of cases per 100,000 people in the population per year. Incidence studies are important for identifying risk factors as well as providing information on prognosis. Most incidence studies have been carried out in more developed countries and are retrospective, although some recent studies have been population-based prospective studies as recommended by the ILAE Commission on Epidemiology (1997).

Studies documenting the incidence of a first epileptic seizure (per 100,000) are shown in Table 1. 


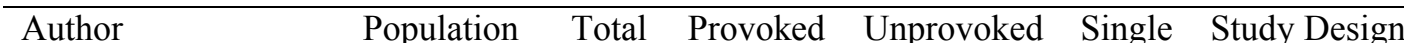

(reference)

\begin{tabular}{lllllll}
\hline France & $1,128,164$ & 71.3 & 29.0 & 42.3 & 18.3 & Prospective
\end{tabular}

(Loiseau et al. 1990)

$\begin{array}{lllllll}\text { USA } & 2,003,357 & 100 & 35.0 & 61.0 & \text { NA } & \text { Retrospective }\end{array}$

(Hauser et al. 1993)

$\begin{array}{lllllll}\text { Sweden } & 101,583 & 76.0 & 20.0 & 56.0 & \text { NA } & \text { Prospective }\end{array}$

(Forsgren et al.1996)

$\begin{array}{lllllll}\text { Switzerland } & 384,657 & 70.8 & 25.2 & 45.6 & \text { NA } & \text { Prospective }\end{array}$

(Jallon et al. 1997)

$\begin{array}{lllllll}\text { Martinique } & 383,596 & 80.5 & 16.4 & 64.1 & \text { NA } & \text { Prospective }\end{array}$

(Jallon et al. 1999)

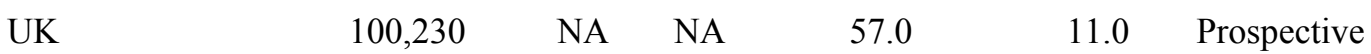

(MacDonald et al.

2000)

Iceland

$272,512 \quad$ NA $\quad$ NA $\quad 56.8$

23.5 Prospective

(Olafsson et al.2005)

USA

270,677 NA NA

40.3

16.4 Prospective

(Benn et al. 2008)

Sweden

$998,500 \quad$ NA NA

33.9

14.4 Prospective

(Adelöw et al. 2009)

Table 1: Incidence of first epileptic seizure (per 100,000 people)

\subsubsection{Incidence of epilepsy in more developed countries}

Incidence studies of epilepsy have been carried in many European countries as well as in the USA with remarkable consistency across different countries. In general, the incidence of epilepsy in the western world is taken to be around 50 (range 40 to 70 ) per 
100,000/year (Sander, 2003). In a systemic review of incidence studies, 40 studies were identified, of which nine were prospective and seven were from resource-poor countries. The median annual incidence of epilepsy was 47.4 per 100,000 and of unprovoked seizures was 56 per 100,000. When the analysis was limited to studies of the highest quality, the median annual incidence of epilepsy decreased to 45 per 100,000 and for unprovoked seizures to 50.8 per 100,000 (Kotsopoulos et al., 2002). In a systemic review of European epidemiological studies, annual incidence rates ranged from 43 to 47 per 100,000 person years (Forsgren et al., 2005a). Two more recent studies provide annual incidences of 33.3 per 100,000 (Iceland; Olafsson et al., 2005) and 68.8 per 100,000 (Denmark; Christensen et al., 2007b).

A summary of the main prospective incidence studies in developed countries are shown in Table 2.

Incidence studies in resource-poor countries are shown in Table 3. The incidence of the main epilepsy syndromes are summarised in Table 4. 


\begin{tabular}{|c|c|c|c|c|}
\hline Authors & Country & $\begin{array}{l}\text { Number } \\
\text { of cases }\end{array}$ & $\begin{array}{l}\text { Age-adjusted } \\
\text { incidence rate per } \\
100,000\end{array}$ & Comments \\
\hline $\begin{array}{l}\text { Forsgren } \\
(1990)\end{array}$ & Sweden & $\begin{array}{l}67 \\
40(\mathrm{SS})\end{array}$ & $\begin{array}{l}33.6^{*} \\
23.2^{* *}\end{array}$ & $\begin{array}{l}\text { Population study (1985-87) } \\
\text { Ages } \geq 17\end{array}$ \\
\hline $\begin{array}{l}\text { Hauser et al. } \\
\text { (1993) }\end{array}$ & US & 880 & 44 & $\begin{array}{l}\text { Comprehensive medical } \\
\text { review (1935-84) }\end{array}$ \\
\hline $\begin{array}{l}\text { Sidenvall et al. } \\
\text { (1993) }\end{array}$ & Sweden & 61 & 73 & $\begin{array}{l}\text { Ages } 0-15 \\
\text { All children with newly } \\
\text { diagnosed within a } 20 \\
\text { month period }\end{array}$ \\
\hline $\begin{array}{l}\text { Forsgren et al. } \\
\text { (1996a) }\end{array}$ & Sweden & 160 & 56 & $\begin{array}{l}\text { Population register } \\
\text { SS Included } \\
\text { Age }>16\end{array}$ \\
\hline $\begin{array}{l}\text { Olafsson et al. } \\
\text { (1996) }\end{array}$ & Iceland & 42 & 47 & $\begin{array}{l}\text { Medical record review } \\
\text { (1993) }\end{array}$ \\
\hline $\begin{array}{l}\text { Zarrelli et al. } \\
\text { (1999) }\end{array}$ & US & 157 & 52.3 & $\begin{array}{l}\text { Comprehensive medical } \\
\text { review }(1980-84)\end{array}$ \\
\hline $\begin{array}{l}\text { Annegers et al. } \\
\text { (1999) }\end{array}$ & US & $\begin{array}{l}197 \\
275(\mathrm{SS})\end{array}$ & $\begin{array}{l}35.5 \\
50.9(\mathrm{SS})\end{array}$ & $\begin{array}{l}\text { HMO population (1988-94) } \\
\text { Ages 0-64 years }\end{array}$ \\
\hline $\begin{array}{l}\text { Beilmann et al. } \\
\text { (1999) }\end{array}$ & Estonia & 216 & 45 & $\begin{array}{l}\text { 1995-1997 } \\
\text { Ages } 1 \text { month }-19 \text { years }\end{array}$ \\
\hline $\begin{array}{l}\text { Oun et al. } \\
(2003)\end{array}$ & Estonia & 81 & 35.4 & $\begin{array}{l}\text { Database review (1994-97) } \\
\text { Ages } \geq 20\end{array}$ \\
\hline $\begin{array}{l}\text { Olafsson et al. } \\
\text { (2005) }\end{array}$ & Iceland & $\begin{array}{l}294 \\
207(\mathrm{SS})\end{array}$ & $\begin{array}{l}32.4 \\
22.8(\mathrm{SS})\end{array}$ & $\begin{array}{l}\text { Nationwide surveillance } \\
\text { system (1995-99) }\end{array}$ \\
\hline $\begin{array}{l}\text { Christensen et al. } \\
\text { (2007) }\end{array}$ & Denmark & 88,616 & 68.8 & $\begin{array}{l}\text { Database review (1977- } \\
\text { 2002) } \\
\text { All ages }\end{array}$ \\
\hline $\begin{array}{l}\text { Adelow et al. } \\
\text { (2009) }\end{array}$ & Sweden & $\begin{array}{l}585 \\
430(\mathrm{SS})\end{array}$ & $33.9^{*}$ & $\begin{array}{l}\text { Incidence Registry (2001- } \\
04) \\
\text { All ages }\end{array}$ \\
\hline
\end{tabular}

Table 2: Prospective population-based incidence studies

$\mathrm{SS}=$ Single Seizure $\mathrm{HMO}=$ Health maintenance organisation

* All first unprovoked seizures, ${ }^{* *}$ Single and acute symptomatic seizures 


\begin{tabular}{|c|c|c|c|c|}
\hline Authors & Country & $\begin{array}{l}\text { Number of } \\
\text { cases }\end{array}$ & $\begin{array}{l}\text { Age-adjusted } \\
\text { incidence rate per } \\
100,000\end{array}$ & Comments \\
\hline $\begin{array}{l}\text { Li et al } \\
(1985)\end{array}$ & China & 16 & 35 & $\begin{array}{l}\text { Small number. Wide } \\
\text { confidence interval }\end{array}$ \\
\hline $\begin{array}{l}\text { Placencia et al. } \\
\text { (1992) }\end{array}$ & Ecuador & 137 & $122-190$ & $\begin{array}{l}\text { Well designed study. } \\
\text { Wide geographical } \\
\text { variation }\end{array}$ \\
\hline $\begin{array}{l}\text { Lavados et al. } \\
\text { (1992) }\end{array}$ & Chile & 102 & 113 & \\
\hline $\begin{array}{l}\text { Rwiza et al. } \\
\text { (1992) }\end{array}$ & Tanzania & 122 & 55 & $\begin{array}{l}\text { Crude incidence } 73.3 \text { per } \\
100,000\end{array}$ \\
\hline $\begin{array}{l}\text { Tekle-Haimanot } \\
\text { et al. (1997) }\end{array}$ & Ethiopia & 139 & 49 & $\begin{array}{l}\text { Crude incidence } 64 \text { per } \\
100,000\end{array}$ \\
\hline $\begin{array}{l}\text { Kaiser et al. } \\
\text { (1998) }\end{array}$ & Uganda & 80 & 156 & $\begin{array}{l}\text { Crude incidence } 215 \text { per } \\
100,000\end{array}$ \\
\hline $\begin{array}{l}\text { Mani et al. } \\
\text { (1998) }\end{array}$ & India & 32 & 49.3 & \\
\hline $\begin{array}{l}\text { Sawhney et al. } \\
\text { (1999) }\end{array}$ & India & 34 & 60.0 & \\
\hline $\begin{array}{l}\text { Wang et al. } \\
(2002)\end{array}$ & China & 16 & 28.8 & $\begin{array}{l}\text { Door to door survey in } \\
\text { rural areas of } 5 \text { provinces }\end{array}$ \\
\hline $\begin{array}{l}\text { Dogui et al. } \\
\text { (2003) }\end{array}$ & Tunisia & 157 & 102.1 & Paediatric study \\
\hline $\begin{array}{l}\text { Idro et al. } \\
\text { (2008) }\end{array}$ & Kenya & 900 & 425 & $\begin{array}{l}\text { Ages } 0-13 \text { years. } \\
\text { Incidence } 875 \text { per } \\
100,000 \text { in children }<5\end{array}$ \\
\hline $\begin{array}{l}\text { Mung'ala-Odera } \\
\text { et al. (2008) }\end{array}$ & Kenya & 39 & 187 & $\begin{array}{l}\text { Incidence calculated for } \\
\text { children aged 6-12 years }\end{array}$ \\
\hline $\begin{array}{l}\text { Mignard et al. } \\
\text { (2009) }\end{array}$ & La Réunion & 766 & 115.4 & $\begin{array}{l}\text { Newly diagnosed } \\
\text { epileptic seizures }(17.6 \% \\
\text { provoked) }\end{array}$ \\
\hline
\end{tabular}

Table 3: Incidence studies in resource poor countries 


\begin{tabular}{|c|c|c|c|c|}
\hline & & $\begin{array}{l}\text { South-West France } \\
\text { (Loiseau et al., } \\
\text { 1990) }\end{array}$ & $\begin{array}{l}\text { Rochester, USA } \\
\text { (Zarrelli et al., } \\
\text { 1999) }\end{array}$ & $\begin{array}{l}\text { Iceland } \\
\text { (Olafsson et al., } \\
2005)\end{array}$ \\
\hline \multirow{4}{*}{$\begin{array}{l}\text { Localisation- } \\
\text { related } \\
\text { epilepsies }\end{array}$} & Total & 15.3 & 34.9 & 18.6 \\
\hline & $\begin{array}{l}\text { Idiopathic } \\
\text { partial epilepsies }\end{array}$ & 1.7 & 0.2 & 1.6 \\
\hline & $\begin{array}{l}\text { Symptomatic } \\
\text { partial epilepsies }\end{array}$ & 13.6 & 17.2 & 8.4 \\
\hline & $\begin{array}{l}\text { Cryptogenic } \\
\text { partial epilepsies }\end{array}$ & - & 17.5 & 8.6 \\
\hline \multirow[t]{4}{*}{$\begin{array}{l}\text { Generalised } \\
\text { epilepsies }\end{array}$} & Total & 7.2 & 7.7 & 3.9 \\
\hline & Idiopathic & 6.1 & 3.7 & 3.1 \\
\hline & $\begin{array}{l}\text { Cryptogenic or } \\
\text { symptomatic }\end{array}$ & 1.1 & 1.7 & 0.7 \\
\hline & Symptomatic & - & 2.3 & 0.1 \\
\hline $\begin{array}{l}\text { Epilepsies with } \\
\text { both generalised } \\
\text { and focal } \\
\text { features }\end{array}$ & & - & 1.7 & 0.8 \\
\hline $\begin{array}{l}\text { Epilepsies } \\
\text { without } \\
\text { unequivocal } \\
\text { focal or } \\
\text { generalised } \\
\text { features }\end{array}$ & & 1.9 & 8.0 & 8.5 \\
\hline $\begin{array}{l}\text { Isolated } \\
\text { unprovoked } \\
\text { seizures }\end{array}$ & & 18.3 & - & 22.8 \\
\hline
\end{tabular}

Table 4: Incidences of epileptic syndromes (per 100,000 person-years) 


\subsection{Prevalence}

Prevalence is defined as the number of people with a disease in a defined population as a proportion of the total population and is usually expressed as the number of cases per 1,000 people in the population. Point prevalence is the number of existing cases (usually active epilepsy) in the population at a particular point in time while period prevalence is the prevalence over a particular period (usually one year). Information about a disease's prevalence is important for service provision and planning. As prevalence studies are performed by means of cross-sectional studies they are fairly quick and easy to perform and much less expensive than cohort studies. Consequently a far greater number of prevalence studies than incidence studies have been performed. Most studies are carried out using a door to door survey using a standardised validated screening questionnaire as this is considered the gold standard methodology.

\subsubsection{Prevalence in developed countries}

Prevalence studies of epilepsy have been performed in many European countries, Japan, Australia and USA. Studies have shown a prevalence of active epilepsy of between 4 and 10 per 1,000 (Sander and Shorvon, 1996) although most studies give a prevalence rate of active epilepsy of 4 to 7 per 1,000 (5.7 per 1,000 (Forsgren, 1992), 4.2 per 1,000 (Sidenvall et al., 1996), 4.8 per 1,000 (Olafsson and Hauser, 1999)). The lowest prevalence rate reported in an industrialized country was in Japan at 1.5 per 1,000 although more recent studies from Japan have estimated prevalence rates of active epilepsy more in concordance with other western countries (5.3 per 1,000 (Oka et al., 2006)). A systematic review (Forsgren et al., 2005a) found that the range of prevalence rates in Europe was 3.3 to 7.8 
per 1,000 with a median prevalence of active epilepsy 5.2 per 1,000. European studies with the lowest reported prevalence rates were from Italy; 3.3 per 1,000 in Sicily (Rocca et al., 2001) and 3.01 per 1,000 in the Aeolian Islands (Gallitto et al., 2005) although the authors of both studies suggested that the low reported prevalence rates may have resulted from people's desire to conceal the diagnosis to avoid perceived social disadvantages as a result of prejudice towards people with epilepsy. In the Rochester study the prevalence of active epilepsy, calculated at 10 year intervals over 50 years ranged from 2.7 per 1,000 in 1940 to 6.8 per 1,000 in 1980 (Hauser et al., 1991).

More recent studies using patient reports from Norway (crude prevalence rate 11.7 per 1000; active epilepsy 6.7 per 1,000; Brodtkorb and Sjaastad, 2008) and Ireland (life prevalence 10 per 1,000; treated epilepsy 8.3 to 9 per 1,000; Linehan et al., 2010) suggest higher prevalence rates in some western countries.

The median lifetime prevalence in developed countries has been estimated to be 5.8 per 1,000 (range 2.7 to 12.4 ) with a median prevalence of active epilepsy of 4.9 per 1,000 (range 2.3 to 10.3; Ngugi et al., 2010). 
Studies of the prevalence of epilepsy in resource-poor countries are shown in Table 5.

\begin{tabular}{|c|c|c|c|}
\hline Author & Country & $\begin{array}{l}\text { Prevalence per 1,000 } \\
\text { people (range) }\end{array}$ & Number of cases \\
\hline Placencia et al (1992) & Ecuador & $6.7-8.0$ & 575 \\
\hline Aziz et al (1999) & $\begin{array}{l}\text { Pakistan and } \\
\text { Turkey }\end{array}$ & $\begin{array}{l}9.98 \text { (Pakistan) } \\
7.0 \text { (Turkey) }\end{array}$ & $\begin{array}{l}\text { 24,130 (Pakistan) } \\
\text { 11,497 (Turkey) }\end{array}$ \\
\hline Nicoletti et al (1999) & Bolivia & $\begin{array}{l}12.3 \\
11.1 \text { active epilepsy }\end{array}$ & 124 \\
\hline $\begin{array}{l}\text { Sridharan and } \\
\text { Murthy (1999) }\end{array}$ & India & 5.35 & 3,27 \\
\hline Wang et al (2003) & China & $\begin{array}{l}7.0 \\
4.6 \text { active epilepsy }\end{array}$ & 387 \\
\hline Burneo et al (2005) & $\begin{array}{l}\text { Latin America } \\
(1974-2002)\end{array}$ & $17.8(6-43.2)$ & $\begin{array}{l}\text { Pooled analysis of } 32 \\
\text { prevalence studies }\end{array}$ \\
\hline Medina et al (2005) & $\begin{array}{l}\text { Rural } \\
\text { Honduras }\end{array}$ & 23.3 & 151 \\
\hline Preux et al (2005) & $\begin{array}{l}\text { Sub-Saharan } \\
\text { Africa } \\
(1986-2004)\end{array}$ & $15.0(5.2-70.0)$ & $\begin{array}{l}\text { Pooled analysis of } 26 \text { door } \\
\text { to door surveys in } 15 \\
\text { countries. All but } 3 \text { rural } \\
\text { populations }\end{array}$ \\
\hline Ndoye et al (2005) & Senegal & 14.2 & 64 \\
\hline Dent et al (2005) & Tanzania & 7.4 active epilepsy & 42 \\
\hline Almu et al (2006) & Ethiopia & 29.5 & 82 \\
\hline $\begin{array}{l}\text { Velez \& Cobos } \\
(2006)\end{array}$ & Columbia & $\begin{array}{l}11.3(9.2-13.8) \\
10.1(8.1-12.4)\end{array}$ & 92 \\
\hline Mac et al (2007) & $\begin{array}{l}\text { Asia } \\
(1985-2006)\end{array}$ & $6.0(1.5-14.0)$ & $\begin{array}{l}\text { Pooled analysis of } 20 \\
\text { studies from } 12 \text { countries }\end{array}$ \\
\hline Edwards et al (2008) & Kenya & 4.5 active epilepsy & 466 \\
\hline (Tuan et al., 2008) & Vietnam & 4.4 active epilepsy & 206 \\
\hline
\end{tabular}

Table 5: Selected prevalence studies from resource-poor countries 


\subsection{Risk factors for epilepsy}

The relative frequencies of specific aetiologies of any chronic condition are best determined by use of population-based studies of newly diagnosed cases, with incidence studies preferable to prevalence studies. Prevalence studies of active epilepsy will miss many cases at both ends of the severity spectrum (the severe cases with causes associated with increased mortality and the more benign cases with causes leading to early remission). Unprovoked seizures or epilepsy are divided into different categories by presumed causation; remote symptomatic, progressive symptomatic, idiopathic (presumed genetic) and cryptogenic or unknown. Some studies, particularly from resource-poor countries, also include people with provoked seizures (acute symptomatic seizures) in studies of epilepsy thereby artificially increasing incidence and prevalence figures.

In population-based studies the epilepsy is most frequently cryptogenic or idiopathic, ranging from $44.5 \%$ (Oun et al., 2003) to $67 \%$ (Olafsson et al., 2005), with the proportion of other causes determined increasing with age. The number of cases classified as cryptogenic has remained broadly similar over the past twenty years despite significant improvements in neuro-imaging. In a recent study in New York (Benn et al., 2008) 55\% of people were defined as having idiopathic/cryptogenic epilepsy, similar to the $61 \%$ in the NGPSE (Sander et al., 1990) almost twenty years previously. Risk factors or associated factors linked to the subsequent development of epilepsy, such as cerebro-vascular disease, head trauma, neurodegenerative disease, CNS infections, neoplasms and learning disability, predominate in identified aetiologies in population-based incidence studies. Aetiologies tend to be age-specific with cerebral palsy, congenital brain damage and learning disability 
predominating in the young while tumours, neurodegenerative disorders and especially cerebrovascular disease dominate in the elderly.

\subsubsection{Neoplasms}

Neoplasms are found to be the cause of seizures in four percent of people with epilepsy (Olafsson et al., 2005). At least thirty percent of people with brain tumours will develop epilepsy, which for many is the first clinical presentation of the underlying malignancy (Hauser et al., 1993). Slow growing tumours, such as low-grade astrocytomas, appear to be more epileptogenic than high grade tumours although this may be related to their increased survival times (Herman, 2002). Seizures occur in 20 to $35 \%$ of those with cerebral metastasis (Herman, 2002).

\subsubsection{Stroke}

Stroke is the most commonly identified antecedent factor in population-based studies, accounting for up to $30 \%$ of cases overall (Forsgren et al., 1996a), with a higher rate in the elderly (44\%; Ruggles et al., 2001).

The overall incidence of seizures is two to three percent within the first 24 hours following an acute stoke (Burn et al., 1997; Szaflarski et al., 2008), 4 to 6\% in the first week (Labovitz et al., 2001; So et al., 1996) and 5\% in the first two weeks (Bladin et al., 2000). Risk factors for the development of early seizures (within two weeks) are anterior hemisphere location (Burn et al., 1997; So et al., 1996), cortical or lobar location of infarction (Bladin et al., 2000; Labovitz et al., 2001), stroke severity (Bladin et al., 2000; Burn et al., 1997), cardio-embolic versus small or large vessel aetiology, younger age and pre-existing disability (Szaflarski et al., 2008). 
Patients with haemorrhagic stroke (intra-cranial haemorrhage (ICH)/Sub-arachnoid haemorrhage $(\mathrm{SAH}))$ have an almost 2-fold increase in risk of seizure compared with the general population (HR1.85; 95\% CI 1.26, 2.73) (Bladin et al., 2000). One study found that the risk of seizure following $\mathrm{ICH} / \mathrm{SAH}$ was $8.4 \%$ compared with $2.9 \%$ for those with infarction (Szaflarski et al., 2008) while another found that the risk of seizures in those with $\mathrm{ICH} / \mathrm{SAH}$ was increased compared with infarction (hazard ratio for $\mathrm{ICH}$ vs cerebral infarction 10.2; 95\% CI 3.7,27.9) (Burn et al., 1997).

The risks of early seizures following acute stroke differ by location and stroke subtype and in one study were $1 \%$ for deep infarction, $4 \%$ for deep ICH, $6 \%$ for lobar infarction, $8 \%$ for SAH and $14 \%$ for lobar ICH. One quarter of those with early seizures had status epilepticus (Labovitz et al., 2001). In a recent review of seizures and ischaemic stroke (Menon and Shorvon, 2009), the most consistent risk factors identified for the development of post-ischaemic stroke epilepsy were cortical involvement, stroke severity (or disability) and male gender.

The cumulative risk of late seizures after stroke is $3 \%$ at one year, $4.7 \%$ at two years, $7.4 \%$ at five years and $8.9 \%$ by ten years (So et al., 1996). Risk factors for the subsequent development of epilepsy are early seizures and stroke recurrence, (So et al., 1996) and late onset (after two weeks) of seizures was found to be an independent risk for the development of epilepsy after ischaemic but not haemorrhagic stroke (Bladin et al., 2000). Overall $10-12 \%$ of stroke survivors will have had a seizure by five years of follow-up, with $36 \%$ of those with early seizures going on to develop epilepsy. Only $3 \%$ of those seizurefree at one month had a seizure between one month and five years (Burn et al., 1997). For those with SAH 7\% had developed epilepsy at 12 months follow-up (4\% had a single seizure). Independent factors associated with the development of epilepsy following SAH 
were presence of subdural hematoma (OR 9.9, 95\% CI 1.9, 52.8) or cerebral infarction (OR 3.9, 95\% CI 1.4, 11.3) (Claassen et al., 2003). After 11 years of follow-up, 25\% of those with SAH had developed epilepsy, all within the first four years. $70 \%$ of those with early seizures developed epilepsy which was more frequent in those with severe neurological deficits than in those without (48\% vs 20\%) (Olafsson et al., 2000).

\subsubsection{Neurodegenerative disorders}

Degenerative disease, most notably Alzheimer's disease (AD), is a commonly identified risk factor for seizures in population-based studies, typically responsible for 5-6\% of cases overall but for a much higher proportion of those over 65 (Olafsson et al., 2005). A diagnosis of $\mathrm{AD}$ or other dementia increases the risk of unprovoked seizures six-fold with no difference in the relative frequencies of complex partial or generalised seizures (Hesdorffer et al., 1996). In a study following people with AD the cumulative incidence of unprovoked seizures at seven years was almost $8 \%$, with an 87 -fold increase in risk of unprovoked seizures in those aged 50-59 and a 3-fold increase in those aged 85+. Independent risk factors for seizures identified on multivariate analysis were younger age, more severe dementia, African-American ethnicity and focal epileptiform features on EEG (Amatniek et al., 2006). Overall 3.6\% of people on an AD register had seizures, the majority of whom (72\%) had complex partial seizures. Most (79\%) had an excellent response to anti-epileptic medications (Rao et al., 2009).

\subsubsection{Trauma}

Head trauma is a significant risk factor for the development of seizures; $5.5 \%$ of incident seizures in the Rochester study were caused by trauma (Hauser et al., 1993). The risk of 
posttraumatic seizures is related to the severity of the injury; the risk of seizures following severe head injury (loss of consciousness and/or post-traumatic amnesia greater than 24 hours) was $7.1 \%$ at one year and $11.5 \%$ at five years. (The risk of post-traumatic epilepsy is significantly higher in open head injuries as a result of missiles). The risk was $0.7 \%$ at one year and $1.6 \%$ at five years after moderate head injury (skull fracture or loss of consciousness and/or post-traumatic amnesia between 30 minutes and 24 hours) (Annegers et al., 1980). A further study from the Mayo clinic looked at the risk of the development of seizures following traumatic brain injury over ten years. The risk of seizures was related to the severity of head injury and decreased over time. Overall, head injury confers a threefold increased risk of seizures compared with the general population. Multivariate analysis showed that early seizures (occurring in the first week after injury) were not predictive of further seizures (Annegers et al., 1988). Significant risk factors for the subsequent development of post-traumatic seizures found on multivariate analysis were the presence of subdural haematoma, skull fracture, loss of consciousness and/or post-traumatic amnesia for greater than 24 hours and age over 65 years. The majority (77\%) of those who develop seizures after a head injury have recurrent seizures. The risk of post-traumatic seizures was highest in the early years following the trauma with the five-year cumulative risk for seizures following mild (loss of consciousness and/or post-traumatic amnesia less than 30 minutes: $0.7 \%)$, moderate $(1.2 \%)$ and severe $(10 \%)$ head injuries. The risk of developing seizures returned to that of the general population five years after mild injury and ten years following moderate injuries while remaining higher after ten years for those with severe injuries (Annegers et al., 1998).

A recent study from Denmark (Christensen et al., 2009) found that the risk of seizures following a head injury persisted beyond ten years with a RR of 7.4 (95\% CI: $6.2,8.9)$ following a severe head injury and $2.2(95 \% \mathrm{CI}: 2.1,2.4)$ following a mild head injury. The 
risk of post-traumatic seizures was higher in females and increased with age following mild and severe injuries. People with a family history of epilepsy were at significantly higher risk of developing seizures following mild $(\mathrm{RR}=5.8,95 \% \mathrm{CI}: 4.6,7.3)$ and severe $(\mathrm{RR}=10.1,95 \% \mathrm{CI}: 4.2,24.3)$ injuries, emphasising the fact that the causation of epilepsy is often multi-factorial.

\subsubsection{Seizures and bacterial meningitis/encephalitis}

Seizures are common in the setting of acute infection accounting for $15 \%$ of those with acute symptomatic seizures in Rochester (Annegers et al., 1995). While infections are the identified aetiology in only a small number of cases $(2.5 \%$ in Rochester; Hauser et al., 1993 ) in the developed world, their importance as an aetiological factor is far greater in resource-poor countries.

In a population-based study, the 20 -year risk of unprovoked seizures following encephalitis or meningitis was $6.8 \%$ overall. The risk was highest in the first five years (Standardised Morbidity Rate (SMR) 10.3) but remained elevated throughout follow-up. The cumulative risk of unprovoked seizures varied with the type of CNS infection and the presence or absence of seizures in the acute setting of the infection. The 20-year risk of developing unprovoked seizures was highest in those with viral encephalitis, $22 \%$ in people with early seizures and $10 \%$ in those without early seizures. In people with bacterial meningitis who survived, the risk of unprovoked seizures was $13 \%$ with and $2.4 \%$ without early seizures. The 20-year cumulative risk of seizures following aseptic meningitis was not significantly increased (2.4\%) over the general population rate (Annegers et al., 1988). 


\subsubsection{Seizures and other CNS infections and infestations}

Malaria is a major problem worldwide affecting millions annually, particularly in resourcepoor countries. In a matched case-control study of 296 patients with cerebral malaria (CM) in Gabon (Ngoungou et al., 2006b), the adjusted odds ratio for developing epilepsy after CM was $3.9(95 \%$ CI $1.7,8.9)$ with additional risk factors identified as positive family history of epilepsy and antecedent history of febrile convulsions. In a similar study from Mali of 110 people with a history of CM and 222 controls (Ngoungou et al., 2006a), the incidence of epilepsy was 1,700 per 100,000 person-years for those who had CM compared with 180 per 100,000 person-years for those with no history of CM. The age-adjusted relative risk for developing epilepsy was $14.3(95 \% \mathrm{CI}, 1.6,13.2 ; \mathrm{p}=0.01)$ underlying the fact that CM-related epilepsy may be a significant problem in malaria endemic areas. The impact of other infestations such as onchocerciasis (caused by the filarial helminth Onchocerca volvulus) has also been noted in areas such as West Uganda where there was a marked difference in the incidence of epilepsy in high endemic compared with low endemic areas (232 compared with 77 per 100,000) (Kaiser et al., 1998).

Neurocysticercosis (NCC), caused by the larvae of the pork tapeworm Taenia solium, while uncommon in the western world, has a very high prevalence in the developing world where it is considered one of the major risk factors for the development of epilepsy (Commission on Tropical Diseases of the ILAE, 1994). There was an association between seropositivity (antibodies against T. Solium) and epilepsy with an odds ratio for epilepsy of 1.85 (95\% $\mathrm{CI}, 0.99,3.4)$ for all cases, with a stronger association in those with partial seizures (OR $2.75,95 \%$ CI $1.44,6.61)$ and late onset seizures (15 years and older, OR 3.66, 95\% CI 1.1, 12.1) in a study in rural Bolivia (Nicoletti et al., 2002). In rural Peru, seropositivity for 
NCC was associated with seizures (OR 2.14, 95\% CI 1.08, 4.2) (Montano et al., 2005) while in India NCC was found to be the cause of active epilepsy in almost one third of people with seizures with no significant differences between rural and urban areas in the prevalence of NCC causing active epilepsy (Rajshekhar et al., 2006).

A study of the association between toxocariasis (caused by the roundworm Toxocara canis in dogs) and epilepsy found a positive association between seropositivity for T. Canis and epilepsy for all cases (OR 2.7, 95\% CI 1.41, 5.19) with a much stronger association for those with late onset partial seizures (OR 18.22, 95\% CI 2.1, 158.1) (Nicoletti et al., 2008).

\subsubsection{Learning disability and cerebral palsy}

Epilepsy is a common problem in those with learning disability (LD) with the risk and severity of epilepsy increasing with the degree of the cognitive deficit. Prevalence ranges from six percent amongst children with mild LD (IQ 50-70) to $24 \%$ in severe LD (IQ < 50) and $50 \%$ in those with profound LD (IQ <20) (Lhatoo and Sander, 2001). In eight percent of cases identified in the Rochester study, the seizures were attributed to LD or cerebral palsy (CP). The cumulative risk of epilepsy in children with LD was $21 \%$ by the age of 22 with the risk five-fold greater in those with severe LD than in those with mild LD (Airaksinen et al., 2000).

In a prospective study of children with LD followed from birth in Scotland (Goulden et al., 1991), the cumulative risk of epilepsy by age 22 was $15 \%$ overall. In children with LD without associated disabilities the cumulative risk was 5.2\% while for children with LD and associated CP the cumulative risk was 38\%. The highest risk of epilepsy was for children with LD associated with a postnatal injury, at 66\% (10 and also 15 years after injury). 
In a study examining the prevalence of epilepsy in adults with and without LD in two primary care practices in the US (McDermott et al., 2005), the prevalence of epilepsy was one percent for the general population, $13 \%$ for those with $\mathrm{CP}, 14 \%$ for those with Down Syndrome, $25.4 \%$ for those with autism, $25.5 \%$ in those with LD alone, and $40 \%$ for those with both LD and CP. The prevalence of epilepsy decreased with increasing age in patients with CP and LD while increasing in people with Down syndrome and the general population.

The risk of epilepsy varies with different subtypes of CP. A study using the CP registry in Norway found that $28 \%$ of children with CP had active epilepsy and $31 \%$ had LD. $19 \%$ of children with unilateral CP had active epilepsy compared with $30 \%$ of children with spastic bilateral CP $(p=0.05)$ and $42 \%$ of children with dyskinetic $C P(p=0.07)$ (Andersen et al., 2008). 


\subsection{Prognosis}

For people with seizures, prognosis is the risk of further seizures after a single unprovoked seizure or the likelihood of achieving seizure freedom or terminal remission after a pattern of recurring seizures has been established (Sander, 1993). In discussing the prognosis of epilepsy, various aspects need to be considered: the likelihood of recurrence following a single seizure, the impact of early versus late treatment, the probability of relapse after prolonged remission, following epilepsy surgery or following anti-epileptic medication withdrawal and the associated morbidity and mortality associated with epilepsy.

\subsubsection{Recurrence after a single seizure}

Prospective population studies of single seizures are difficult as many seizures go unrecognised or are un-witnessed and in some instances individuals do not present to medical attention unless the seizure is convulsive. The overall risk of recurrence following a single seizure has been reported as 27 to $71 \%$. A meta-analysis of 13 studies found that the average risk of seizure recurrence was $40 \%$ in prospective studies and $52 \%$ in retrospective studies (Berg and Shinnar, 1991). The time interval between the seizure and inclusion in a follow-up study of people with single seizures influences the recurrence rate, as many people have a recurrence within weeks of the index seizure, and are therefore classified as having epilepsy, thus artificially lowering the estimated recurrence rate following a single seizure (Hopkins et al., 1988). The risk of subsequent seizures decreases with time, with up to $80 \%$ of recurrences occurring within two years of the initial seizure; recurrence rates are $36 \%$ in prospective studies and $47 \%$ in retrospective studies at two years (Berg and Shinnar, 1991). In the NGPSE, 67\% of those with a single seizure had a recurrence within 12 months and 78\% within 36 months (Hart et al., 1990). 
In a prospective study of 407 children with a first unprovoked seizure, $182(45 \%)$ had a second seizure with a median time to recurrence of 6.2 months. The cumulative risk of a second seizure was 22\% (6 months), 29\% (one year), 37\% (two years), $43 \%$ (five years) and $46 \%$ (ten years) (Shinnar et al., 2000). In the Dutch study of epilepsy in childhood (Stroink et al., 1998) 156 children, none of whom received treatment, were followed up from the time of a first unprovoked seizure; $54 \%$ had had a recurrence by two years.

Similarly another study (Lindsten et al., 2001b) found a recurrence rate of $58 \%$ at 750 days in 107 people aged 17 or older. No person who was free of seizures in the first 750 days after the index seizure had a recurrence thereafter during a median follow-up of 10.3 years, underlying the impression that the risk of seizure recurrence is highest in the first one to two years following the seizure.

\subsubsection{Recurrence after a second seizure}

The risk of recurrent seizures following a second seizure was investigated in a predominantly adult population (Hauser et al., 1998). In those patients with a second seizure, the risk of a further seizure was $32 \%$ at three months, $41 \%$ at six months, $57 \%$ at one year and $74 \%$ at four years. Of those who did not have a recurrence after the second seizure within the first four years of follow-up, none had a relapse in the subsequent three years. The majority $(63 \%)$ of those with a third seizure had a further seizure with the risk of a fourth seizure being $31 \%$ at three months, $48 \%$ at six months, $61 \%$ at one year and $78 \%$ at three years. As with single seizures, the risk of further seizures is highest immediately after the last one. Similarly for children, the risk of a third seizure was $57 \%$ at one year, $63 \%$ at two years and $72 \%$ at five years after having a second seizure (Shinnar et al., 2000). 


\subsubsection{Short and medium term prognosis}

It is usually accepted that the prognosis of epilepsy is generally favourable with $60-70 \%$ achieving remission (Sander, 1993). In a prospective study of children with newly diagnosed epilepsy followed for a median of five years from the time of diagnosis, $74 \%$ had achieved a period of remission ( $\geq$ two years seizure freedom), following which $24 \%$ had a further seizure. In those who had a relapse, approximately $50 \%$ occurred when antiepileptic medication was being withdrawn or had been stopped (Berg et al., 2001). In the NGPSE after nine years, $86 \%$ of those with definite epilepsy had achieved a remission of three years and $68 \%$ a remission of five years. Sixty eight percent were in three year terminal remission and 54\% in five year terminal remission by nine years (Cockerell et al., 1997). In a study of 107 patients aged $\geq 17$ with newly diagnosed epilepsy, at ten years follow-up the cumulative remission rates were $68 \%$ (one year), $64 \%$ (three years) and $58 \%$ (five years) (Lindsten et al., 2001a).

\subsubsection{Long -term prognosis}

Few studies have looked at the long-term prognosis of people with epilepsy and most are retrospective.

In the Rochester study (Annegers et al., 1979a), 65\% had achieved a five year period of remission at ten year follow-up and $76 \%$ at twenty years. At ten years after diagnosis $61 \%$ were in terminal remission, with $70 \%$ in terminal remission at 20 years. Of people in remission, $20 \%$ remained on anti-epileptic medication while $50 \%$ had successfully discontinued medication and remained seizure-free for five or more years. A cohort of children with active epilepsy was followed for 12 years at which stage $64 \%$ were in 
terminal remission (defined as three or more years seizure free) (Brorson and Wranne, 1987).

Of 144 children with epilepsy followed for an average of 37 years, $67 \%$ were in terminal remission, on or off medication at end of follow-up. Early remission, occurring within the first year of treatment was achieved by 45 (31\%), and the remission continued to terminal remission in 23 (51\%) of these. Remission without relapse occurred in another 72 (50\%) with a mean delay of nine years. Twenty (14\%) entered remission but subsequently relapsed with further remission indicating a relapse-remitting pattern while 27 (19\%) continued with seizures from the onset (Sillanpaa and Schmidt, 2006a).

Of 102 children from the same cohort followed up for a median of 40 years, $95(93 \%)$ had one or more periods of remission (one year) (Sillanpaa and Schmidt, 2009), emphasising the overall excellent prognosis of childhood epilepsy. One of the major aims of this thesis is to examine the long-term prognosis after 25 years in a prospective incident cohort with regard to premature mortality and seizure outcomes. Outcomes in long-term studies are summarised in Table 6. 


\begin{tabular}{|c|c|c|c|c|c|}
\hline $\begin{array}{l}\text { Author } \\
\text { (publication year) }\end{array}$ & $\begin{array}{l}\text { Number } \\
\text { in cohort }\end{array}$ & $\begin{array}{l}\text { Follow-up } \\
\text { (years) }\end{array}$ & $\begin{array}{l}\text { Proportion } \\
\text { 5YR TR } \\
(\%)\end{array}$ & $\begin{array}{l}\text { Proportion } \\
\text { 5YR TR off } \\
\text { AEDs }(\%)\end{array}$ & $\begin{array}{l}\text { Study } \\
\text { Design }\end{array}$ \\
\hline $\begin{array}{l}\text { Annegers et al } \\
(1979)\end{array}$ & 457 & 20 & $70 \%$ & $50 \%$ & $\begin{array}{l}\text { Historic Incident } \\
\text { Cohort }\end{array}$ \\
\hline $\begin{array}{l}\text { Okuma et al } \\
\text { (1981) }\end{array}$ & 1,868 & 10 & $58.3 \%$ & $\mathrm{~N} / \mathrm{A}$ & $\begin{array}{l}\text { Retrospective } \\
\text { Multi-institutional } \\
\text { study (P) }\end{array}$ \\
\hline $\begin{array}{l}\text { Brorson and } \\
\text { Wranne (1987) }\end{array}$ & 194 & 12 & $64 \%$ & $40 \%$ & Retrospective (P) \\
\hline Oka et al (1989) & 730 & $10-15$ & $79.1 \%$ & $\mathrm{~N} / \mathrm{A}$ & Retrospective (P) \\
\hline $\begin{array}{l}\text { Wakamoto et al } \\
(2000)\end{array}$ & 143 & 18.9 & $62.8 \%$ & $54.7 \%$ & Retrospective (P) \\
\hline $\begin{array}{l}\text { Lindsten et al } \\
(2001)\end{array}$ & 107 & 10 & $64 \%$ & $17.5 \%$ & $\begin{array}{l}\text { Prospective }(\text { All }> \\
17 \text { years })\end{array}$ \\
\hline $\begin{array}{l}\text { Sillanpaa and } \\
\text { Schmidt (2006) }\end{array}$ & 144 & 40 & $67 \%$ & $58 \%$ & Prospective $(\mathrm{P})$ \\
\hline $\begin{array}{l}\text { Geerts et al } \\
(2010)\end{array}$ & 413 & 14.8 & $70.9 \%$ & $61.9 \%$ & Prospective $(\mathrm{P})$ \\
\hline
\end{tabular}

$\mathrm{P}=$ Paediatric Study; 5YR TR=5 year terminal remission rate

Table 6: Long-Term Follow-up Studies in Epilepsy 


\subsubsection{Prognostic factors}

Many studies have looked at possible predictors of seizure prognosis, including age of onset, gender, aetiology, seizure type, EEG patterns, number of seizures prior to treatment and early response to treatment (Sander, 2003). In people presenting with a first-ever seizure, the presence of multiple discrete seizures within 24 hours is not associated with a worse prognosis compared with those with a single seizure (Kho et al., 2006). Remote symptomatic epilepsy, the presence of a neurological birth deficit (which was associated with a $100 \%$ relapse rate in the NGPSE (Hart et al., 1990)) and learning disability have been consistently shown to be associated with a poorer prognosis. In one study (Brorson and Wranne, 1987) the three year remission rate was $89 \%$ for those with idiopathic epilepsy and normal examination compared with only $49 \%$ in those with a neurological deficit or learning disability. The number of seizures in the first six months after onset has been found to be a strong determinant of the probability of subsequent remission with $95 \%$ of those with two seizures in the first six months achieving a five year remission compared with only $24 \%$ of those with more than ten seizures (MacDonald et al., 2000). A high initial seizure frequency, focal EEG slowing and acute symptomatic seizures or neonatal status epilepticus have all been shown to be significantly associated with an increased risk of developing refractory or intractable epilepsy in children (Berg et al 2001). Seizure type has been an inconsistent prognostic factor with some studies indicating that those with partial seizures have a poorer prognosis (Annegers et al., 1979a) while other studies have demonstrated a poorer prognosis for people with generalised onset seizures (Shafer et al., 1988). Individuals with multiple seizure types, as is typical in the childhood encephalopathies, appear to have a poorer prognosis (Collaborative Group for the Study of Epilepsy, 1992). 
A significant reduction or complete cessation of seizures within three months of initiating treatment has been shown to be a strong predictor of subsequent remission (Sillanpaa et al., 1998). The probability of seizure remission decreases significantly with each successive treatment failure (Kwan and Brodie, 2000). Only 11\% of patients who discontinued the first appropriate anti-epileptic drug (AED) due to lack of efficacy became seizure free on a second AED and only $4 \%$ on a third medication or on polypharmacy. This figure is, however, contentious and one of the major themes of this thesis is the examination of the impact of medication changes in a cohort of people with apparent pharmaco-resistant epilepsy followed over several years.

Children who experience clusters of seizures during treatment were much more likely to have refractory epilepsy than children without clusters $(42 \%$ vs $13 \%, \mathrm{p}=0.01)$ and less likely to achieve five year terminal remission $(p=0.004)$ (Sillanpaa and Schmidt, 2008). Children who continued to have weekly seizures during the first year of treatment had an eight-fold increase in the risk of developing intractable epilepsy and a two-fold increase in risk of never achieving one year terminal remission. (Sillanpaa and Schmidt, 2009)

\subsubsection{The impact of aetiology on prognosis}

When comparing the prognosis of people with epilepsy by aetiology (epilepsy syndrome), people with idiopathic generalized epilepsy appear to have a better prognosis than those with symptomatic or cryptogenic partial epilepsy. Most (82\%) people with idiopathic generalised seizures achieved one year seizure freedom compared with only $35 \%$ with symptomatic partial epilepsy and $45 \%$ with cryptogenic partial epilepsy (Semah et al., 1998). Temporal lobe epilepsy (TLE) was associated with a poorer prognosis than extratemporal lobe epilepsy (36\% vs 20\% seizure free) (Semah et al., 1998). In people with a 
single identified lesion, TLE with hippocampal sclerosis (HS) had a particularly poor prognosis $(11 \%$ seizure free $)$ compared with other aetiologies $(24 \%$ with cortical dysplasia (CD) seizure free). Individuals with HS and another identified pathology (dual pathology) had the worst prognosis (3\% seizure free) (Semah et al., 1998). In contrast, others found no difference in prognosis between those with symptomatic and cryptogenic partial epilepsy ( $58 \%$ vs $56 \%$ at least 1 year seizure free). Comparing patients by aetiology, they found that mesial TLE had the worst prognosis ( $42 \%$ seizure free) with seizure freedom rates of 54\% (CD), 55\% (cerebral atrophy), 57\% (cortical gliosis), 63\% (cerebral infarctions) and 78\% (arterio-venous malformations) (Stephen et al., 2001).

Children with remote symptomatic epilepsy or abnormal neurological development have a poorer prognosis and are at high risk of developing intractable epilepsy (Berg et al., 1996; Sillanpaa, 1993).

\subsubsection{The impact of anti-epileptic drugs on prognosis}

In the western world most patients are commenced on anti-epileptic drugs (AEDs) after two unprovoked seizures implying that prognostic studies from western countries are essentially those of treated epilepsy. Evidence from studies from resource-poor countries where a significant treatment gap exists, suggests that many people enter spontaneous remission with no anti-epileptic medication (Sander, 1993; Placencia et al., 1994; Nicoletti et al., 2009), and moreover that the response to AEDs in patients with chronic longstanding epilepsy is comparable to that of patients with new-onset seizures (Feksi et al., 1991; Watts, 1992; Placencia et al., 1994). Such evidence implies that the view that epilepsy is a chronic progressive condition (Gowers, 1881) is over-pessimistic. It has been suggested that people with epilepsy can be sub-divided into prognostic groups based on their aetiology and 
epileptic syndrome, which in turn determines their need and response to anti-epileptic treatment (Sander, 1993).

Two studies have assessed the impact of medication on the risk of recurrence after a single seizure. In the FIRST study, people with a first unprovoked generalised seizure were randomised to either immediate treatment (treated group) or to treatment only after a further seizure (untreated group). While immediate treatment reduced the risk of early relapse, it did not affect the long-term prognosis. The two-year remission rate was $72 \%$ in the treated group and $57 \%$ in the untreated group, with rates of $84 \%$ vs $79 \%$ at three years and $85 \%$ vs $86 \%$ at ten years. The comparable probabilities of attaining five-year remission were $47 \%$ vs $40 \%$, at three months, $58 \%$ vs $58 \%$ at two years and 64 vs $64 \%$ at five years (Leone et al., 2006).

In the MESS study (Marson et al., 2005) people with a single seizure or early epilepsy (all types) were randomised to receive immediate or deferred treatment. People in the immediate treatment group had increased time from the index seizure to a second seizure or a first generalised seizure, in addition to having a reduced time interval to two-year remission. At five-year follow-up, however, $76 \%$ in the immediate group and $77 \%$ in the deferred group had achieved three to five years seizure freedom. (Marson et al., 2005) On the basis of the MESS study data, possible predictors of seizure recurrence were identified. The number of seizures (all types), an abnormal EEG and the presence of a neurological deficit were all significant risk factors for subsequent seizures with the risk increasing when any of these factors occurred in combination (Kim et al., 2006). In summary, these studies have concluded that immediate treatment delays the early recurrence of seizures but does not affect the medium or long term prognosis. 


\subsubsection{Prognosis following AED withdrawal}

In the largest randomised control trial of continued treatment versus drug withdrawal in 1,013 patients in remission (two or more years seizure free), at two years postrandomization $41 \%$ of those who had discontinued medication had had a recurrence of seizures compared with $22 \%$ of those who stayed on medication. The difference in relapse rates between the two groups was maximal at nine months with the rate of relapse being higher in the discontinuation group in the first two years of follow-up, but by two-four years the risk of relapse was higher in those continuing treatment (MRC AED Withdrawal Study Group, 1991). Those who experienced a relapse were followed up and by three years 95\% who experienced a relapse had a further one year remission and by five years, $90 \%$ had had a further two year remission period. This indicates that the long-term prognosis is similar in those who relapsed and in those who did not have a relapse within two years following AED withdrawal. .

A further analysis of the data from the MRC AED withdrawal study using regression modelling has recently been reported (Bonnett et al., 2011). The recurrence risk within the first 12 months following AED withdrawal was 30\% (95\% CI 25, 35) while the risk of recurrence within the next 12 months for those remaining seizure-free at three months after AED withdrawal was $15 \%(95 \%$ CI 10,19$)$ and for those still seizure-free at six months was nine percent $(95 \%$ CI 5,13$)$. For those who had had a seizure recurrence and who then restarted AED treatment, after three months of seizure freedom after recommencing treatment the risk of seizure recurrence within the next 12 months was $26 \%$ (95\% CI 17 , 35) while at 6 months seizure freedom after treatment reinstatement, the risk of recurrence in the next 12 months was 18\% (95\% CI 10, 27) (Bonnett et al., 2011). 
An analysis of 14 AED withdrawal studies (Schmidt and Loscher, 2005) found that the recurrence rate following AED discontinuation ranged from 12 to $66 \%$ (mean $34 \%$; 95\% CI: 27, 43) and reinstatement of treatment was successful in obtaining further remission in, on average, $80 \%(95 \% \mathrm{CI}: 75,85)$ with no significant differences between age groups. A second remission may, however, take many years to achieve while in an average of $19 \%$ (95\% CI: $15,24 \%)$ the reintroduction of the medication did not control the seizures as before. Up to $23 \%$ of those discontinuing treatment go on to develop intractable epilepsy. Risk factors for subsequent poor treatment outcome were symptomatic focal epilepsy and cognitive deficits (Schmidt and Loscher, 2005).

Ninety children who discontinued treatment following remission were followed for an average of 32 years from seizure onset (Sillanpaa and Schmidt, 2006b). Seizure relapse occurred in $37 \%$ (33) with $36 \%$ of relapses occurring within the first year, $46 \%$ within the second year and $67 \%$ within the third year. The last relapse occurred after 28 years followup. Eight opted to restart treatment with two achieving subsequent five-year terminal remission with a delay of 10-19 years after restarting treatment. The other six individuals did not achieve five-year terminal remission, with two considered to have drug-resistant epilepsy. Factors associated with failure to achieve five-year terminal remission following reinstatement of treatment were symptomatic and localisation related aetiology (Sillanpaa and Schmidt, 2006b).

Despite the risk of seizure recurrence, people with epilepsy may choose to discontinue treatment because of the impact of continuing anti-epileptic medication on quality of life. In one study (the Akershus study), the effect of AED withdrawal on quality of life was assessed. At one-year seizure recurrence had occurred in $15 \%$ of the withdrawal group compared with $7 \%$ in the non-withdrawal group (RR 2.46; 95\% CI: 0.85, 7.08). The proportion of people having completely normal neuropsychological findings increased from 
$11 \%$ to $28 \%$ in the withdrawal group - while decreasing from $11 \%$ to $9 \%$ in the nonwithdrawal group. (Fifteen neuropsychological tests were applied examining immediate and delayed verbal and visual memory; attention and psychomotor speed, executive functions such as verbal fluency, response inhibition and divided attention; simple reaction time with both hands and choice reaction time with lexical and figurative stimuli; motor speed and complex coordination with both hands). No differences in quality of life were observed between the two groups. At 41 months follow-up, predictors of continued seizure freedom following treatment withdrawal were prior use of carbamazepine (approximately three-fold increase in likelihood of remaining seizure-free compared with people on any other drug (OR 2.86; 95\% CI 1.3, 6.3)) and a normal neurological examination (Lossius et al., 2008).

\subsubsection{Patterns of relapse and remission in epilepsy}

Seizures continue in up to a third of people despite treatment. For the majority of these, new AEDs offer the only hope of seizure freedom as only a few are candidates for epilepsy surgery (Lhatoo et al., 2003). In high-income countries most people are treated after presentation of the first or second seizure and so any assessment of the natural history of epilepsy is that of treated epilepsy (Goodridge and Shorvon 1983; Sander and Shorvon 1987; Sillanpaa and Schmidt 2006; Berg et al., 2009; Geerts et al., 2010). Studies from resource-poor countries where access to treatment is often limited allow a limited examination of untreated epilepsy or the "natural history of epilepsy". These studies suggest that at least a third of people with epilepsy spontaneously enter remission without treatment (Watts 1992; Sander 1993, Placencia et al., 1994; Kwan and Sander 2004; Nicoletti et al., 2009). 
It has been suggested that refractory or pharmaco-resistant epilepsy is progressive and therefore pre-determined, with early identification of such cases possible (Kwan and Brodie 2000; Kwan and Brodie 2002). Others have argued that pharmaco-resistance may not always manifest early in the course of the epilepsy but can sometimes develop later (Berg et al., 2006; Sillanpaa and Schmidt 2006). It has also been suggested that in a minority of people the course of epilepsy may alternate between periods of relapse and remission (Goodridge and Shorvon1983; Shorvon and Sander, 1986; Berg et al., 2004; Sillanpaa and Schmidt 2006), but it has not been clearly established whether, once a particular seizure pattern has been established, it will persist. Indeed the relative frequencies of different seizure patterns in people with chronic epilepsy have not been clearly established. Early recognition of factors that may predispose people to a seizure pattern of recurrent remissions and relapses is important; as such people could be counselled against future trials of AED withdrawals following a period of prolonged seizure freedom. Identification of such factors if possible, is one of the aims of this thesis.

\subsubsection{The prognosis for those with intractable epilepsy}

It has been suggested that failure to control seizures with the first or second AED implies that the probability of subsequent seizure control with further AEDs is slim (Kwan and Brodie, 2000). This can inevitably lend to clinical nihilism when dealing with such people in clinic. A recent series of papers suggests that such a view is overly pessimistic. In a retrospective study of the effect of 265 medication changes in 155 people with uncontrolled epilepsy of at least five years duration, $16 \%$ of all people were rendered seizure free (12 months or more) following a drug introduction while a further $21 \%$ had a reduction of 
seizure frequency of $50-99 \%$. Overall $28 \%$ of the cohort was rendered seizure free by medical changes (Luciano and Shorvon, 2007).

It had previously been shown that the likelihood of seizure remission in children with LD and intractable seizures is less $(1.5 \%$ per year) than in children of normal intelligence and intractable seizures (4\% per year) (Huttenlocher and Hapke, 1990).

A group of 246 people with refractory epilepsy was followed for three years. Excluding 11 $(4.5 \%)$ who became seizure free because of surgery, $26(11 \%)$ became seizure free (sixmonths terminal remission) as a result of medication change (addition of a new AED or dose change). No single AED was associated with a statistically significant probability of inducing seizure freedom. People with LD were statistically less likely to achieve a remission (7.2\% vs $18.1 \%)$. Overall approximately five percent of people became seizure free per year, highlighting the fact that irrespective of the number of AED previously tried, there is still a possibility of inducing meaningful seizure remission in this population (Callaghan et al., 2007).

The probability of seizure relapse following remission was retrospectively studied in a cohort of 186 people with intractable epilepsy who were followed for a median of 3.8 years. Overall 20 people achieved a remission of $\geq 12$ months with four percent becoming seizure-free per year. Of these, five subsequently suffered a relapse with the estimated cumulative probability of relapse $33 \%$ at two years and $44 \%$ at three years. No clear predictors of remission or subsequent relapse were identified (Choi et al., 2008). In summary approximately four to five percent of people with refractory epilepsy will achieve a remission of twelve months per year following AED change, many of whom will subsequently relapse. It is certainly possible that new therapies (over 13 AEDs in the past 20 years) have improved the prognosis of epilepsy and that therefore the often cited figure 
that approximately one third of people will have pharmaco-resistant epilepsy may be overly pessimistic, not to mention outdated. More medium- to long-term follow-up of people with apparent pharmaco-resistant epilepsy are needed to confirm or refute such a hypothesis. 


\subsection{Mortality}

People who have been diagnosed with epilepsy need to know whether this will have any impact on their life expectancy. It is now accepted that people with epilepsy have a two to three-fold risk of dying earlier compared with the general population. This increased risk of premature death is stratified by aetiology and it has been consistently shown that children/adults with neurological deficits and symptomatic (acute and remote) seizures have a significantly increased risk of premature death while the risk for those with idiopathic or cryptogenic epilepsy appears to be broadly similar to that of the general population.

\subsubsection{Methodological issues}

Mortality studies in epilepsy should be community-based studies of people with incident epilepsy. Studies of people with prevalent epilepsy may under-estimate the short-term mortality (as the mortality in people with epilepsy has consistently been shown to be highest in the early years following diagnosis) while simultaneously overestimating the long-term mortality (as those who have gone into remission may not be included in the cohort). The risk of premature death in people with epilepsy has been studied using death certificates, hospital or institutional records and through follow-up of community cohorts. Death certificates have been shown to be an unreliable source, with epilepsy being recorded on the death certificate in only seven percent of people known to have epilepsy (Bell et al., 2004). This figure increased to $17 \%$ in people with frequent seizures. In a community based study of mortality in children with epilepsy, epilepsy was recorded on the death certificate in $55 \%$ of deaths directly attributable to epilepsy (Harvey et al., 1993). 
Other studies have used hospital or institution registries with the inherent risk of selection bias of more severe cases and therefore an overestimation of the mortality risk.

The most commonly reported measure of mortality is the SMR which is defined as the ratio of the observed deaths in the cohort divided by the number of expected deaths if the agesex specific rates were the same as those of the standard population. This is an external comparison in cohort design studies and measures how much more (or less) likely a person in the study population is to die during a certain period of time than someone of the same age and sex in the standard population.

The direct comparison of SMRs in different studies is problematic as the SMR is calculated by indirect standardisation using the age and gender distribution of the group with epilepsy, which may differ among studies. It has been argued that these potential differences may invalidate direct comparison of SMRs derived from distinct populations (Logroscino and Hesdorffer, 2005). Even taking this in account, some insight may be derived by comparing SMRs between studies of similar populations as a crude measure of changes of mortality between different time periods particularly as this is the only method of comparison available.

The proportionate mortality rate (PMR) is not a direct measure of mortality but rather gives the proportion of deaths due to one specific cause. This is the measure of mortality used in case controlled studies and is influenced by both an increase of one cause of death but also by decreases of other causes.

Other measures used are the mortality rate (MR) which is the incidence rate of mortality in the cohort and the case fatality $(\mathrm{CF})$ which is the proportion of the cohort dying during a specific time period (Logroscino and Hesdorffer, 2005). 


\subsubsection{Mortality following a newly diagnosed seizure}

A review of mortality following a single seizure estimated that the SMR in people with a newly diagnosed unprovoked seizure ranged from 2.5 to 4.1 with the highest SMRs in young children and in those with symptomatic seizures (Hauser and Beghi, 2008). In the

Gironde study the SMR at one year following a single seizure was 4.1 (95\% CI 2.5, 6.2) (Loiseau et al., 1999). Mortality risk was significantly increased in people with acute symptomatic seizures (SMR 10.3; 95\% CI 8.3, 12.7), remote symptomatic seizures (SMR 6.4; 95\% CI 3.6, 10.3) and progressive symptomatic seizures (SMR 19.8; 95\% CI 14.0, 27.3) but not in people with idiopathic or cryptogenic epilepsy.

In a Swedish population-based cohort study of adults the SMR was 2.5 (95\% CI 1.2, 3.2) in people with a newly diagnosed unprovoked seizure and was markedly elevated in the first two years after diagnosis (SMR 7.3; 95\% CI 4.4, 12.1) with a second peak at 9 to 11 years (SMR 5.4; 95\% CI 2.7, 11.2) (Lindsten et al., 2000). The SMR was significantly elevated in people with remote symptomatic epilepsy (SMR 3.3; 95\% CI 2.4, 4.5) but not in people with idiopathic epilepsy. The increased risk of mortality was most pronounced in those aged less than 60 .

In a prospective study of 407 children with a single unprovoked seizure followed for a mean of 14.2 years, it was found that treatment following a single seizure did not alter the mortality rate (Shinnar et al., 2005).

In the NGPSE cohort, $15 \%(83 / 564)$ of people with definite seizures were classified as having acute symptomatic seizures with an SMR of 3.0 (95\% CI 2.0, 4.3) at 11-14 years following diagnosis (Lhatoo et al., 2001). The 30 day CF in the Rochester study after an acute symptomatic seizure was $19.2 \%$ with an SMR of $149.1(95 \%$ CI $119.9,184.7)$ (Hesdorffer and D'Amelio, 2005). 


\subsubsection{Mortality in cohort studies}

The mortality of people with epilepsy has been studied in hospital based cohorts (Mohanraj et al., 2006; Nilsson et al., 1997; Shackleton et al., 1999), institutionalised cohorts (Klenerman et al., 1993; White et al., 1979) and population-based cohorts (Camfield et al., 2002; Ding et al., 2006; Hauser et al., 1980; Lhatoo et al., 2001; Lindsten et al., 2000; Olafsson et al., 1998). Reported SMRs in mortality studies from developed countries range from 1.6 to 4.1 (Forsgren et al., 2005).

Much of the variation in reported SMRs in people with epilepsy is dependent on the source population. In general, studies from institutions report the highest SMRs, with the lowest coming from general population-based studies. In 21 identified studies, studies from institutions showed wider variation in mortality rates (SMR 1.9 to 5.1) than populationbased studies (SMR 1.3 to 3.1) (Shackleton et al., 2002).

\subsubsection{Temporal trends in mortality in people with epilepsy}

It is generally accepted that people with epilepsy are at increased risk of premature death compared with the general population (Cockerell et al., 1994; Hauser et al., 1980; Tomson, 2000; Gaitatzis et al., 2004), but it has not been clearly established how this risk changes over the course of the condition. It is assumed that most early deaths in epilepsy are due to the underlying cause of the epilepsy, whereas any later increase in mortality rate is primarily due to the epilepsy itself (Forsgren et al., 2005; Hauser et al., 1980), despite the fact that the majority of people become seizure free. If this is the case, one would expect to see evidence of decreasing mortality rates attributable to epilepsy in general population studies over the past 50 years and in particular in the past 20 years when more treatment options for epilepsy have become available. One of the aims of this thesis is to examine 
how the risk of premature mortality changes over the course of epilepsy with particular reference to the long-term mortality in people in the NGPSE cohort.

\subsubsection{The impact of age and gender on mortality}

Most studies have shown that males have a higher premature mortality risk than females although no clear explanation for this has been demonstrated. Age also influences the risk with reported SMRs demonstrating an inverse relationship with age. The SMR tends to be high in children but this relates principally to the underlying cause of the epilepsy (remote symptomatic, peri-natal insults) rather than to the epilepsy itself.

In a Finnish cohort of 245 children with epilepsy identified between 1961 and 1964 and followed up prospectively (Sillanpaa et al., 1998), 44 had died by the end of follow-up in 1992 of whom $75 \%$ of had remote symptomatic epilepsy with a similarly high representation of remote symptomatic epilepsy in childhood mortality studies from Australia (Harvey et al 1993) and Nova Scotia. (Camfield et al., 2002). Of those who died, $89 \%$ were not in remission at the time of death, with a relative risk of death in those with active epilepsy compared with those in remission of 9.26 (95\% CI 3.8, 22.7). The cause of death was definitely or proabably related to a seizure in $45 \%$ (20) of cases. There were three cases of sudden unexplained death in epilepsy (SUDEP) in people with idiopathic epilepsy, none of whom was in remission at the time of death (Sillanpaa et al., 1998). In the extended follow-up of the cohort up to 2002, 60 (24\%) had died, of whom 51 (85\%) were not in terminal remission ( $\geq$ five years seizure free) at the time of death. Those with a remote symptomatic aetiology were three times as likely to die as those with idiopathic/cryptogenic aetiologies (37\% vs $12 \%, \mathrm{P}<0.001)$. Of the 60 deaths, 33 (55\%) 
were felt to be epilepsy-related including 18 deaths from SUDEP, giving a cumulative risk of SUDEP of seven percent at 40 years $(12 \%$ for those not in terminal remission (Sillanpaa and Shinnar, 2010).

Decreasing SMRs are found with increasing age groups. The lowest SMRs are reported in the $75+$ age group; this relates in part to the fact that this age group has a high mortality rate (Hauser et al., 1993).

Epilepsy is a very important non-obstetric cause of mortality in pregnant women with epilepsy although the number certainly represents only a small proportion of deaths in women with epilepsy. Epilepsy-related mortality in pregnancy was recently highlighted at the $29^{\text {th }}$ International Epilepsy Congress in Rome in September 2011 as an area in which further research is needed.

\subsubsection{Causes of mortality}

Causes of death in people with epilepsy can be divided into epilepsy related and nonepilepsy related deaths. For people with symptomatic epilepsy (both remote and progressive) the excess mortality risk relates primarily to the underlying cause of the epilepsy rather than to the epilepsy itself. In a study of 692 children with epilepsy followed up over an average of 13 years, the SMR was 5.3 with functional neurological deficit being the only independent predictor of mortality (occurring in $85 \%$ of cases) (Camfield et al., 2002). In a Dutch study of mortality in people with epilepsy followed up over 40 years the SMR was 16 in the first two years decreasing to 2.8 thereafter (Shackleton et al., 1999). After two years, approximately one third of deaths were directly or indirectly attributable to epilepsy. 
The importance of the underlying cause as a contributor to the mortality rate in people with epilepsy, particularly in the early years following diagnosis, is demonstrated in the Gironde study where the causes of death after one year were the underlying pathology in $64 \%$, an unrelated cause in 20\%, unknown in $9 \%$ and seizure-related in $6 \%$. There were no reported deaths in people with idiopathic generalised epilepsy. (Loiseau et al., 1999)

Common non-epileptic causes of mortality cited in mortality studies include pneumonia, cerebrovascular disease, malignancy and heart disease. SMRs and PMRs are consistently elevated for these causes in the population-based studies and often markedly so in the first few years of follow-up. In a Swedish study looking at cause specific mortality in over 9,000 adults with epilepsy, the overall SMR was $3.6(95 \%$ CI 3.5, 3.7) with SMRs being increased for specific causes such as cancer (SMR 2.6; 95\% CI 2.4, 2.8), respiratory disease (SMR 4.0; 95\% CI 3.6, 4.5), heart and cerebrovascular disease (SMR 3.1; 95\% CI 3.0, 3.3) and accidents and poisoning (SMR 5.6; 95\% CI 5.0, 6.3) (Nilsson et al 1997). The risk of premature death with heart disease was found to be elevated in those aged 25 to 64 but not for those aged 65 years and over in the Rochester cohort (Annegers et al., 1984). Bronchopneumonia is an important cause of mortality in people with epilepsy of all ages, not just the elderly and was associated with the highest SMR (7.2) in the NGPSE (Lhatoo et al., 2001). This may be related to aspiration during seizures but this is unproven.

The influence of LD and epilepsy was investigated in a Swedish study. The SMR was 1.6 $(95 \%$ CI $1.3,2.0)$ in people with LD only but this increased to $5.0(95 \%$ CI $3.3,7.5)$ for those with LD and epilepsy, with the increase in mortality associated with seizure type and frequency. (Forsgren et al., 1996c) 
In studies from institutions and hospitals where people have presumably more severe epilepsy, epilepsy-related deaths are more common. In one study, PMRs were cancer (26\%), bronchopneumonia (25\%), circulatory diseases $(24 \%)$, seizure-related deaths (other than SUDEP) (12\%) and SUDEP (6\%) (Klenerman et al., 1993).

SMRs and PMRs for cancer have been consistently elevated in people with epilepsy even after excluding CNS neoplasms. Cancer mortality was compared between two cohorts with epilepsy, one from an institution with more severe epilepsy and the other a community based population with milder epilepsy. The SMRs for all cancers was elevated in the cohort with severe epilepsy (SMR 1.42; 95\% CI 1.18, 1.69) but not in the milder group (SMR 0.93; 95\% CI 0.84, 1.03). The SMR for brain and CNS neoplasms was significantly elevated in the group with milder epilepsy (Singh et al., 2009).

\subsubsection{Epilepsy-related deaths}

Deaths directly related to epilepsy include SUDEP, status epilepticus, accidents as a consequence of a seizure (including drowning and aspiration pneumonia), iatrogenic (drug toxicity and idiosyncratic) and suicide.

\subsubsection{SUDEP}

SUDEP is defined as a sudden, unexpected death in an individual with or without evidence of a seizure where post-mortem does not reveal a specific cause of death (Lhatoo and Sander, 2005). Estimates of SUDEP rates are heavily influenced by the population under study with much higher rates in those with severe or refractory epilepsy. Identified risk factors for SUDEP include younger age of onset, long duration of epilepsy and refractory epilepsy (Tomson et al., 2005). In a prospective cohort study, the incidence of SUDEP was 
$1.21 / 1000$ patient years and accounted for $18 \%$ of all deaths. Independent risk factors identified for SUDEP were the occurrence of tonic-clonic seizures, LD (IQ < 70) and treatment with greater than two AEDs, after adjustment for seizure frequency (Walczak et al., 2001; Langan et al., 2005). The incidence of SUDEP was 0.35 per 1000 person years in the Rochester cohort (Ficker et al., 1998) while an incidence of 1:295/year was found in children with more severe epilepsy and LD (Nashef et al., 1995).

\subsubsection{Accidents}

People with epilepsy may die as a result of an accident during a seizure. Based on attendance records of four Accident \& Emergency (A\&E) Departments, the risk of injury as a result of a seizure was estimated to be 29.5 per 100,000 population per year (Kirby and Sadler, 1995). Many seizure-related injuries tend to be minor with an increased risk related to background seizure frequency (Lawn et al., 2004), but some injuries can be fatal. In a one year population-based study (using inpatient records, doctors' claims and A\&E visits) the annual incidence of injuries was higher in people with epilepsy after adjusting for age, gender and co-morbidities, with $20.6 \%$ of people with epilepsy having at least one injury compared with $16.1 \%$ among people without epilepsy $(\mathrm{p}<0.001)$. In particular people with epilepsy were more likely to have fractures, crushing injuries, intracranial and other head injuries (Kwon et al., 2010).

Similarly over a two-year period, people with epilepsy were more likely to have injuries inflicted on them by others (OR 1.46; 95\% CI 1.04, 2.03) after adjustment for comorbidities but were not more likely to have motor vehicle accidents or completed or attempted suicide (Kwon et al., 2011). 


\subsubsection{Drowning}

People with epilepsy have an increased risk of drowning (15 to 19 fold) compared with the general population. In a meta-analysis of the risk of drowning, the total SMR was 18.7. The SMR varied depending on the population under study, with an SMR of 5.4 in communitybased incident cohorts, 18 in people with prevalent epilepsy, 25.7 in people with epilepsy and learning disability and 96.9 for people in institutional care (Bell et al., 2008).

\subsubsection{Suicide}

People with epilepsy have been shown to be at increased risk of suicide in some studies (Nilsson et al., 1997; Zielinski, 1974b) but not in others (Hauser et al., 1980; Lhatoo et al., 2001). In a meta-analysis, the SMRs for people with epilepsy and suicide were markedly elevated, particularly for those with temporal lobe epilepsy (Harris and Barraclough, 1997). In a population-based controlled study from Denmark, $2.3 \%$ of people with epilepsy, committed suicide compared with $0.74 \%$ in the general population corresponding to a 3fold increased risk (Rate Ratio 3.17; 95\% CI 2.88, 3.50). This risk was particularly high in people with co-morbid psychiatric illness and in the first six months following diagnosis (Christensen et al., 2007a). A recent meta-analysis found that the overall SMR for suicide in people with epilepsy was 3.3 (95\% CI 2.8, 3.7), with the highest rates being in those post temporal lobe excision (SMR 13.9), following other forms of epilepsy surgery (SMR 6.4) and in people with temporal lobe epilepsy (SMR 6.4) (Bell et al., 2009a).

\subsubsection{Mortality and surgery}

A cohort of 299 people was retrospectively followed after temporal lobe surgery to establish early and late mortality (Hennessy et al., 1999). Overall 20 people died, providing 
a mortality rate of 1 per 136 person-years and an SMR of 4.5 (95\% CI 3.2, 6.6). Three deaths were the result of surgical complications; if these are excluded the mortality rate is 1 per 161 persons-years and the SMR 3.7 (95\% CI 2.5, 5.3). The mortality associated with right-sided MTS was markedly elevated compared with left sided MTS (SMR 32.0 vs 3.3). This suggests that the mortality rate associated with epilepsy surgery is in the order of one percent and that epilepsy surgery reduces but does not eliminate the excess mortality associated with epilepsy.

In a prospective study of people with refractory partial epilepsy evaluated for epilepsy surgery at a single centre during a 19 year period, non-surgical patients were twice as likely to die during the follow-up period (HR $2.5 ; 95 \%$ CI $1.5,4.4$ ) and more likely to die from a probable epilepsy-related cause (SUDEP, drowning, status epilepticus; $n=24$ ) than the surgery group $(\mathrm{n}=7)(\mathrm{HR} 4.6 ; 95 \%$ CI 1.9, 11.0) (Bell et al., 2010). This supports the view that surgery reduces the risk of premature death in people with refractory partial epilepsy, although it may be that some people who are intrinsically unsuitable for epilepsy surgery may be at the same time at higher risk of epilepsy-related death.

\subsubsection{Anti-epileptic drugs and mortality}

It has been suggested that anti-epileptic treatment with more than two AEDs increases the risk of premature death and in particular of SUDEP (Walczak et al., 2001), though other studies have not shown an increased risk of SUDEP with any AED in monotherapy or in combination (Opeskin and Berkovic, 2003). There has been much debate on the association between AEDs and the risk of suicide since the U.S. Food and Drug Administration (FDA) issued an alert in 2008 as the result of a meta-analysis which found that there was a 1.8-fold increased risk of suicidality associated with all AEDs (Hesdorffer and Kanner, 2009). It 
does however seem that the risk of suicide in people taking AEDs for epilepsy without comorbid depression, although slightly increased, appears to be very low (Bell et al., 2009b; Andersohn et al., 2010). It has been reported that long-term use of AEDs is associated with an increased risk of fractures particularly in women, with the risk increasing with the duration of treatment (Souverein et al., 2006).

Non-adherence to AEDs has been shown to be associated with an over threefold increased risk of death (hazard ratio $3.32 ; 95 \%$ CI $3.11,3.54$ ) after controlling for possible confounding factors. Non-adherence was also associated with $86 \%$ increased of hospital admission and a 50\% increased risk of Emergency Department attendance (Faught et al., 2008).

\subsubsection{Conclusions}

It is clear that a diagnosis of epilepsy is associated with an increased risk of premature death, particularly in the early years following diagnosis. Up to one third of such deaths can be directly or indirectly attributable to epilepsy. This risk is decreased but possibly not eliminated by rendering the person completely seizure free by treatment or surgery. Overall people with epilepsy have been found to have a reduction in life expectancy which is highest at the time of diagnosis and reduces with time. This reduction can be up to two years in people with idiopathic/cryptogenic epilepsy and up to ten years in people with symptomatic epilepsy (Gaitatzis et al., 2004). 


\subsection{Febrile Seizures}

The ILAE defines a febrile seizure as "a seizure occurring in childhood after age one month associated with a febrile illness not caused by an infection of the CNS, without previous neonatal seizures or a previous unprovoked seizure and not meeting criteria for other acute symptomatic seizures" (Commission on Epidemiology and Prognosis ILAE, 1993). This definition is notable for the fact that there is no specified upper age limit, while also differing in the specified lower age limit from the definition of the American Academy of Paediatrics which broadly defines a febrile seizure as "a seizure accompanied by fever without central nervous system infection, occurring in infants and children between six months and five years of age" (Provisional Committee on Quality Improvements, 1996). The definition of febrile seizures adopted throughout this thesis is that of the ILAE. Most studies from Western countries show that febrile seizures occur in two to four percent of children with higher rates reported in studies from Japan and Guam (Hauser, 1994). In the Oakland study, the first large population study of febrile seizures in 18,500 children, two percent had a febrile seizure by the age of five (Van der Berg and Yerushalmy, 1969). A slightly higher rate of febrile seizures was reported in the National Collaborative Perinatal Project (NCPP), where 3.5\% (4.2\% of African-American) of 1,706 children experienced at least one febrile seizure between the ages of one month and seven years (Nelson and Ellenberg, 1978). Similar figures have been reported in children from Rochester (2.3\% by the age of five) (Annegers et al., 1979b), the UK (2.7\%) (Verity and Golding, 1991) and Holland (2.7\% by the age of six) (Verburgh et al., 1992). In contrast a prevalence rate of $8.3 \%$ for three year old children has been reported from Japan (Tsuboi, 1984), which while not immediately explainable, may reflect different cultural (communal) sleeping practices. 
A recent prospective population-based study of the incidence of febrile seizures in Finland found that $6.9 \%$ of children had at least one febrile seizure by the age of five with the authors arguing that the prospective nature of the study may give a more accurate (and higher) rate of febrile seizures in the population than the lower reported rates in retrospective studies in other populations (Sillanpaa et al., 2008).

In the NCCP (and subsequent studies), febrile seizures were subdivided into simple (SFS), and complex types (CFS) (with febrile status epilepticus being a subtype of CFS). A SFS is a generalised seizure without focal features, lasting less than 15 minutes, with no recurrence within 24 hours and resolves spontaneously. CFSs are either longer (greater than 15 minutes), have focal features or recur within 24 hours of the first episodes. SFS represent the majority of febrile seizures in the reported data (70-80\%) (Annegers et al., 1987;Berg et al., 1997; Nelson and Ellenberg, 1978; Verity and Golding, 1991). Approximately one third of children with a febrile seizure have a recurrence and about nine percent have three or more seizures (Annegers et al., 1987;Berg et al., 1992; Nelson and Ellenberg, 1978). Factors such as young age of onset, a history of febrile seizures in a first degree relative, a low recorded temperature in the A\&E department and a short time interval between the onset of the fever and the seizure are all predictors of a higher risk of febrile seizure recurrence (Berg et al., 1997).

\subsubsection{Mortality and febrile seizures}

It is generally accepted that the mortality associated with febrile seizures is very low, with no reported deaths in the 1,706 children with febrile seizures in the NCCP study (Nelson and Ellenberg, 1978) and four deaths in the Oakland study in the 246 children with febrile 
seizures, two of whom died as a result of the infection causing the febrile convulsion and the other two from unrelated causes (Van der Berg and Yerushalmy, 1969). In the NGPSE there were no reported deaths in the cohort of 220 children with febrile seizures followed up for a median of 11.2 years (MacDonald et al., 1999). Similarly none of the 398 children in the UK National Cohort Study who developed febrile seizures died (Verity and Golding, 1991). Overall there appears to be no associated risk of mortality with SFSs or short CFSs. In a recent review on the morbidity and mortality associated with febrile seizures the authors identified four studies where death as an outcome of febrile seizures was reported, ranging from $3.5 \%$ to $12.8 \%$. Moreover in 28 studies with 2,471 children with febrile seizures (SFS and CFSs) there were 21 deaths giving an overall mortality rate of $0.85 \%$. In a sub-analysis of 876 children with febrile status epilepticus (FSE) in 14 studies, there were 18 deaths giving a mortality rate from FSE of $1.6 \%$.(Chungath and Shorvon, 2008). In a population-based cohort study of $1,675,643$ children born in Denmark between $1^{\text {st }}$ January 1977 and $31^{\text {st }}$ December 2004, there were 232 deaths in 55,215 children with a history of febrile seizures. While the long-term mortality was not increased in children with febrile seizures, children with CFSs (but not SFSs) had a $80 \%$ increased risk of mortality in the first year (mortality rate ratio (MMR) $1.80(95 \%$ CI 1.31, 2.4) and a 90\% increased in the second year following a first febrile seizure (MMR 1.89 (95\% CI 1.27, 2.7)) with the rate returning to that of the background population thereafter (Vestergaard et al., 2008).

\subsubsection{Risk of subsequent epilepsy}

There is much debate about the risk of the development of subsequent epilepsy in children with febrile seizures, particularly in light of the supposed risk of hippocampal sclerosis and temporal lobe epilepsy following a febrile seizure. In the NCCP one percent of children 
with SFSs had developed epilepsy by the age of seven. In contrast, two percent of children with CFS, a family history of epilepsy or a pre-existing neurological deficit developed epilepsy, increasing to $10 \%$ if two or more risk factors were present (Nelson and Ellenberg, 1978). In the Oakland study, about three percent of children with febrile seizures subsequently developed afebrile seizures (Van der Berg and Yerushalmy, 1969). In the UK National Cohort Study, nine of the 398 (2.26\%) children with febrile seizures developed epilepsy, six of whom had CFSs $(6.3 \%$ of those with CFSs) compared with only three children with SFSs (1\% of total) (Verity and Golding, 1991).

In the NGPSE, 12 of $208(6 \%)$ had developed epilepsy after a median follow-up of 11.2 years, with cumulative percentages of $1.4 \%(95 \%$ CI $0,2.9)$ by two years, $3.3 \%(95 \%$ CI 0.9. 5.7) by five years and $5.2 \%(95 \%$ CI $2.2,8.3)$ by ten years. Having four or more febrile seizures was associated with a significantly increased risk of the development of subsequent epilepsy (OR $9.4(95 \%$ CI 1.5, 5.7), $\mathrm{p}<0.015)$ compared with the general population (MacDonald et al., 1999). In this thesis, we will examine the long-term risk of developing epilepsy in people with febrile seizures in the NGPSE cohort after 25 years follow-up.

In a prospective population-based cohort study of 1.54 million children born in Denmark between 1978 and 2002, 49,857 children were diagnosed with febrile seizures and 16,481 people were diagnosed with epilepsy, of whom 2,149 (13\%) had a history of febrile seizures. After 23 years follow-up, the cumulative incidence of epilepsy was $6.9 \%$ (95\% CI $6.5,7.3)$ in those with a history of febrile seizures compared with $1.8 \%(95 \%$ CI $1.8,1.9)$ in those without a history of febrile seizures. Overall children with febrile seizures had a six fold increased risk of developing epilepsy (rate ratio 5.98, 95\% CI 5.71, 6.26) (Vestergaard et al., 2007). 
In a recent review on the morbidity and mortality of febrile seizures (Chungath and Shorvon, 2008) 16 studies were identified which looked at the risk of afebrile seizures following a febrile seizure with incidence ranging from $0.25 \%$ to $33 \%$. Of the 4,160 children with febrile seizures, 241 developed afebrile seizures giving a summated rate of 5.8\%. Twenty three studies were identified describing the risk of epilepsy after febrile seizures with the incidence ranging from 0.1 to $32 \%$ in hospital-based studies to a rate of two to seven percent in population-based studies. In total, $544(2.5 \%)$ out of 21,901 children with febrile seizures, developed epilepsy during the follow-up period. Not surprisingly the reported rate of subsequent epilepsy was higher the longer the period of follow-up, with studies with more than ten years follow-up reporting higher rates of epilepsy ( 4.5 to $7.5 \%$ ) than the risk of 2 to $4 \%$ in studies of less than ten years follow-up (Chungath and Shorvon, 2008).

Six studies were identified which examined the risk of developing epilepsy in people with a history of CFSs or FSE. Overall 73 out of 427 people (17.1\%) developed epilepsy with reported incidences ranging from $2.1 \%$ to $32.1 \%$ (Chungath and Shorvon, 2008).

\subsubsection{Conclusions}

The risk of developing subsequent epilepsy is increased in children compared with the general population, particularly in the first few months following the febrile seizure. Overall the risk of epilepsy is approximately $1-2 \%$ for those with SFSs, 4 to $8 \%$ in those with CFSs and probably greater than ten percent in those with SFE.

In contrast, the associated mortality of febrile seizures is very low. Indeed there is no evidence of an increased mortality risk following a SFS or short CFS. The mortality rate in children with a prolonged CFS or SFE is probably less than $1.6 \%$ although the risk of 
premature deaths appears to be slightly elevated in the first two years following the episode. 


\section{$1.8 \quad$ Status epilepticus}

\subsubsection{Introduction}

Status epilepticus (SE) has been famously described as the "maximum development of epilepsy" (Clark and Prout, 1903/4) and is one of the most feared manifestations of epilepsy.

The generally held contention that the underlying aetiology is the major determinant of outcome in SE is supported by a small study in which the effect of treatment on prognosis in 47 people with SE was examined. They found that the outcome was independent of the anaesthetic agent used or the extent of the EEG burst suppression achieved (Rossetti et al., 2005). Similarly in a retrospective study from Serbia of 750 people with SE of whom 120 $(13 \%)$ died, the death was judged to be due to the underlying (or co-morbid) cause in $65.8 \%$ and to a combination of the underlying disease and other aetiologies in a further $22.5 \%$ (Sokic et al., 2009).

Despite these studies, the role of aetiology and other factors such as seizure duration, level of consciousness, age and EEG on the prognosis of status epilepticus in terms of mortality and morbidity has not been examined in a systematic review. It is a contention of this thesis that only pooling the data from all the major studies of status epilepticus (both populationand hospital-based) will allow an accurate appraisal of the relative contributions of the underlying cause and other factors independent of aetiology to outcomes measures in status epilepticus to be obtained. 


\subsubsection{Definition}

Any consideration of the prognosis of status epilepticus requires an examination of the definition. The current definition of status epilepticus derives from the proposals made at the first major meeting devoted to the subject of SE, the $\mathrm{X}^{\text {th }}$ Marseilles Colloquium, held in 1962 and led by Henri Gastaut (Gastaut et al., 1967). At the meeting, a new and influential definition of SE was proposed - a condition characterised by an epileptic seizure, which is so frequently repeated or so prolonged as to create a fixed and lasting epileptic condition. A classification of status epilepticus was subsequently adopted which paralleled the newly formed International Classification of Seizure Type. The ILAE in its revision of the seizure type classification in 1981, revised the definition (in an addendum): The term status epilepticus" is used whenever a seizure persists for a sufficient length of time or is repeated frequently enough that recovery between attacks does not occur. These definitions of SE, excellent as they are, beg the question "how long should a seizure last before it is categorised as SE?", and this lack of definition of a minimum time period has proved problematic from the point of view of clinical research (Shorvon, 1994) and epidemiology, and is the subject of continued debate. The definition of the minimum period of seizure activity before an episode is deemed to be considered status epilepticus clearly impacts on the overall prognosis. Gastaut himself suggested that 60 minutes duration was a reasonable minimum period to consider a seizure 'enduring' and this became the ILAE standard at the time. The suggested minimum time shortened to 30 minutes (Shorvon, 1994), then 20 minutes (Bleck, 1991), to ten minutes (Treiman et al., 1998) and then to five minutes (Lowenstein, 1999). The five minute definition was based on the assumption that convulsive seizures lasting longer than five minutes were seldom self-terminating. Indeed based on their observations of 120 generalised tonic-clonic seizures (GTCSs) in 47 people, 
one group advocated the use of intravenous AEDs if a GTCS persisted longer than two minutes (Theodore et al., 1994). Such a proposition does not seem to be in accord with common clinical experience, where many seizures lasting longer than five minutes are selfterminating without therapy. Indeed this impression is supported by a study in which the time period used in the definition of SE was 30 minutes or more (for continuous seizures or intermittent seizures during which the person did not regain consciousness in between) was compared with prolonged seizure episodes lasting 10 to 29 minutes (DeLorenzo et al., 1999). Almost all (93\%) those with SE required anticonvulsant treatment to control and stop the seizures compared with 57\% of the prolonged seizure group. Moreover the mortality for people with SE was $19 \%$ compared with $2.6 \%$ for the prolonged seizure group $(p<0.001)$ and $0 \%$ for those whose seizures stopped spontaneously. The definitions with 30-60 minute minimum durations are based on physiological criteria and the definitions with 5-10 minute minimum durations have been devised for an essentially operational reason - the highly important goal of encouraging the early application of therapy.

The issue of the minimum duration of status epilepticus was discussed at the London Colloquium of Status Epilepticus (April 2007) (Shorvon et al., 2007) where most, but not all, participants still favoured the 30 minute period.

Another term frequently encountered in the literature is 'refractory SE'. There is no universally agreed definition, and different studies have used different definitions. Three factors are commonly used to define 'refractoriness' in convulsive SE - the duration of the status, the number of ineffective therapies and the need for anaesthesia. All are arbitrary. Some authorities have defined SE to be refractory if it is resistant to sub-anaesthetic drug 
therapy. The lack of agreement reflects the inherently unsatisfactory nature of the term, which is probably best avoided (Neligan and Shorvon, 2008).

\subsubsection{The epidemiology of status epilepticus}

To date there have been seven population-based studies of status epilepticus published from Richmond, Virginia (DeLorenzo et al., 1996), Rochester, Minnesota (Hesdorffer et al., 1998a), Switzerland (Coeytaux et al., 2000; Jallon et al., 1999a), Hessen, Germany (Knake et al., 2001), California (Wu et al., 2002), Bologna, Italy (Vignatelli et al., 2003) and London (Chin et al., 2006). All but the Rochester study were prospective and conformed to the ILAE recommendations for epidemiological studies in epilepsy (1993) (ILAE Commission on Epidemiology and Prognosis, 1993). These studies give a minimum incidence of SE of 10-20/100,000. Under-ascertainment of cases is a major problem for all epidemiological studies of epilepsy and SE. The Richmond study gave a much higher estimated incidence of SE of 41-61/100,000. Evidence that the incidence of GCSE requiring hospitalisation may be decreasing is provided by the Californian study (Wu et al., 2002). This study was carried out using a state-wide hospital database to identify all hospitalisations for generalised convulsive status epilepticus in California between 1991 and 1998. Overall the incidence rate for GCSE was 6.2/100,000 population but this figure fell by $42 \%$ between the years 1991 and 1998 from 8.5 to $4.9 / 100,000$. The incidence of SE was slightly higher in males in all studies except the study from Italy (Vignatelli et al., 2003), which showed a slight female preponderance.

Race and ethnicity have been recognised as important factors; the Richmond study found that the incidence of SE was three times higher in the African-American population than in the Caucasian population (DeLorenzo et al., 1996), a finding that was replicated in the 
Californian study (Wu et al., 2002). Age is a dominant factor, with SE incidence forming a U-shaped graph with peaks in the very young and the elderly. 


\begin{tabular}{|c|c|c|c|c|c|c|c|}
\hline & $\begin{array}{l}\text { Richmond } \\
\text { Virginia USA }\end{array}$ & $\begin{array}{l}\text { Rochester, } \\
\text { Minnesota, USA }\end{array}$ & $\begin{array}{l}\text { French speaking } \\
\text { Switzerland }\end{array}$ & Hessen, Germany & Bologna, Italy & $\begin{array}{l}\text { California } \\
\text { USA }\end{array}$ & $\begin{array}{l}\text { London } \\
\text { UK }\end{array}$ \\
\hline Year & 1989-1991 & $1965-1984$ & $1997-1998$ & 1997-1999 & $1999-2000$ & 1991-1998 & $2002-4$ \\
\hline Population & 202,774 & $1,090,055$ & $1,735,420$ & 743,285 & 336,876 & N/A & 605230 \\
\hline Number of cases & 166 & 199 & 172 & 150 & 44 & 19,491 & $\begin{array}{l}226 \text { total } \\
176 \text { first-ever } \\
\text { episode of SE }\end{array}$ \\
\hline $\begin{array}{l}\text { Incidence of SE } \\
\text { (per 100,000 per } \\
\text { year) }\end{array}$ & $\begin{array}{l}41 \text { (raw) } \\
61 \text { (adjusted) }\end{array}$ & 18.3 (adjusted) & $\begin{array}{l}9.9 \text { (raw) } \\
10.3 \text { (adjusted) }\end{array}$ & 15.0 & 13.1 & $6.2(4.9-8.5)$ & $\begin{array}{l}\text { 17-23 (adjusted) } \\
\text { 12.5-14 (adjusted; } \\
\text { first-ever episode) }\end{array}$ \\
\hline $\begin{array}{l}\text { Female: male } \\
\text { ratio }\end{array}$ & $1: 1.2^{1}$ & $1: 1.9^{2}$ & $1: 1.7^{2}$ & $1: 1.9^{3}$ & $1: 0.74^{2}$ & $1: 1.1$ & $1: 1.12$ \\
\hline $\begin{array}{l}\text { History of prior } \\
\text { epilepsy }\end{array}$ & $42 \%$ & $46 \%$ & $42.4 \%$ & $33 \%$ & $39 \%$ & N/A & $7 \%^{4}$ \\
\hline Case fatality & $22 \%(3 \%)^{5}$ & $19 \%$ & $7.6 \%$ & $9.3 \%$ & $39 \%$ & $10.7 \%$ & $3 \%$ \\
\hline Inclusions/Exclu & People aged $\leq$ one & - & Excluding people & Only people aged & Only people aged & Only people with & Only convulsive \\
\hline
\end{tabular}




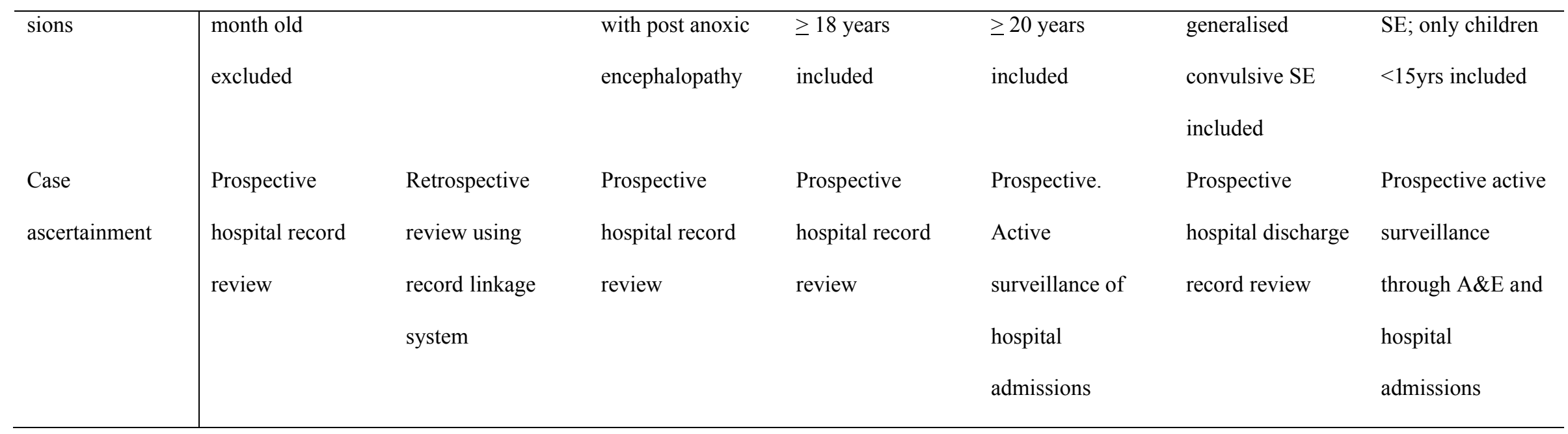

Table 7: Population-based studies of status epilepticus

(Data derived from: DeLorenzo RJ et al 1996; Hesdorffer et al 1998; Coeytaux et al 2000; Knake et al 2001; Wu et al 2002; Vignatelli et al 2003; Chin et al 2006)

FOOTNOTES

$1=$ Raw data

$2=$ Adjusted ratio

$3^{3}=$ Adjusted figures, from the regions with the best case ascertainment (and least likely to selection bias

${ }^{4}=$ Excluding febrile seizures

$5=22 \%$ overall mortality, $3 \%$ mortality in children with SE 


\section{Chapter 2: Aims}

\subsection{The aims of the Systematic Review of the Temporal Trends in Mortality in People with Epilepsy (Study 1) are:}

1. To determine the pattern of change of mortality risk, relative to the general population, in people with epilepsy (as measured by Standardised Mortality Ratios (SMRs)) in the course of their epilepsy by means of longitudinal prospective cohort studies.

2. To determine whether there have been any changes in reported mortality rates for epilepsy over time

The analyses was subdivided according to source populations (population-based, hospitalbased and studies from institutions. 


\subsection{The aims of this extension to the National General Practice Study of} Epilepsy and Epileptic Seizures (NGPSE), a prospective community-based cohort study (Study 2) are:

1. To determine the long-term mortality rates for people with epilepsy and febrile seizures in this cohort, with particular reference to previously reported rates and epilepsy classification at initial assessment

2. To determine the long-term risk $(22+$ years $)$ of the development of non-febrile seizures/epilepsy in people in the cohort with a history of childhood febrile seizures

3. To determine the long-term $(22+$ years $)$ remission and terminal remission rates for people with epilepsy in the cohort 


\subsection{The aims of the Seizure Patterns in Chronic Epilepsy Study (Study} 3) are:

1. To determine the pattern of remission and relapse of epilepsy in a cohort of people with refractory epilepsy, attending a tertiary referral centre

2. To determine whether any clinical or demographic features are predictive of seizure patterns

3. To use the data from this and other studies to develop a remission and relapse model of prognosis of epilepsy 


\subsection{The aims of the Impact of Medication Change in people with}

\section{Chronic Epilepsy Study are:}

1. To determine the seizure outcome at six to seven years follow-up in a cohort of people with drug-resistant epilepsy with AED treatment changes and to compare them with baseline seizure frequency (as measured by the average seizure frequency in the three months prior to AED initiation). 


\subsection{The aims of the Systematic Review of the Prognosis of Status Epilepticus are:}

1. To determine the impact of aetiology on the prognosis of status epilepticus with particular reference to morbidity and mortality

2. To determine the impact of other factors (age, duration of SE, level of consciousness and EEG findings) on the prognosis of status epilepticus with particular reference to morbidity and mortality 


\section{Chapter 3: $\quad$ The Systematic Review of the Temporal Trends in Mortality in people with epilepsy (Study 1)}

\subsection{Methods: Research strategy}

A search of PubMed and Medline for suitable articles was carried out. Initially the terms “epilepsy” AND (“mortality" OR "premature death" OR “death” OR "fatality"), excluding animal studies, were searched and all papers written in English and French were reviewed. This identified 2505 articles and this was reduced by excluding papers whose primary focus was status epilepticus or sudden unexpected death in epilepsy (SUDEP). Titles and abstracts of all relevant articles were reviewed and the bibliographies of key papers were perused to identify other relevant studies. A further search of the literature was performed using the search terms "epilepsy" AND "mortality" AND "trends". 


\subsection{Changes in SMR in people with epilepsy over the course of the condition}

\subsubsection{Population-based cohort studies}

Eight population-based studies of mortality amongst people with epilepsy have been reported (Table 7). Three of these studies (Ding et al., 2006; Loiseau et al., 1999; Zielinski, 1974) have only short follow-up periods, so no conclusions about changes in mortality rates with duration of epilepsy can be drawn from these. The oldest study, a prevalent cohort from Poland, (Zielinski, 1974) had a follow-up of three years, the Gironde study looked at the SMR in people with epilepsy one year after diagnosis (Loiseau et al., 1999), and in the other, a prospective study of a predominantly prevalent cohort in rural China, the median follow-up was 25 months (Ding et al., 2006). 


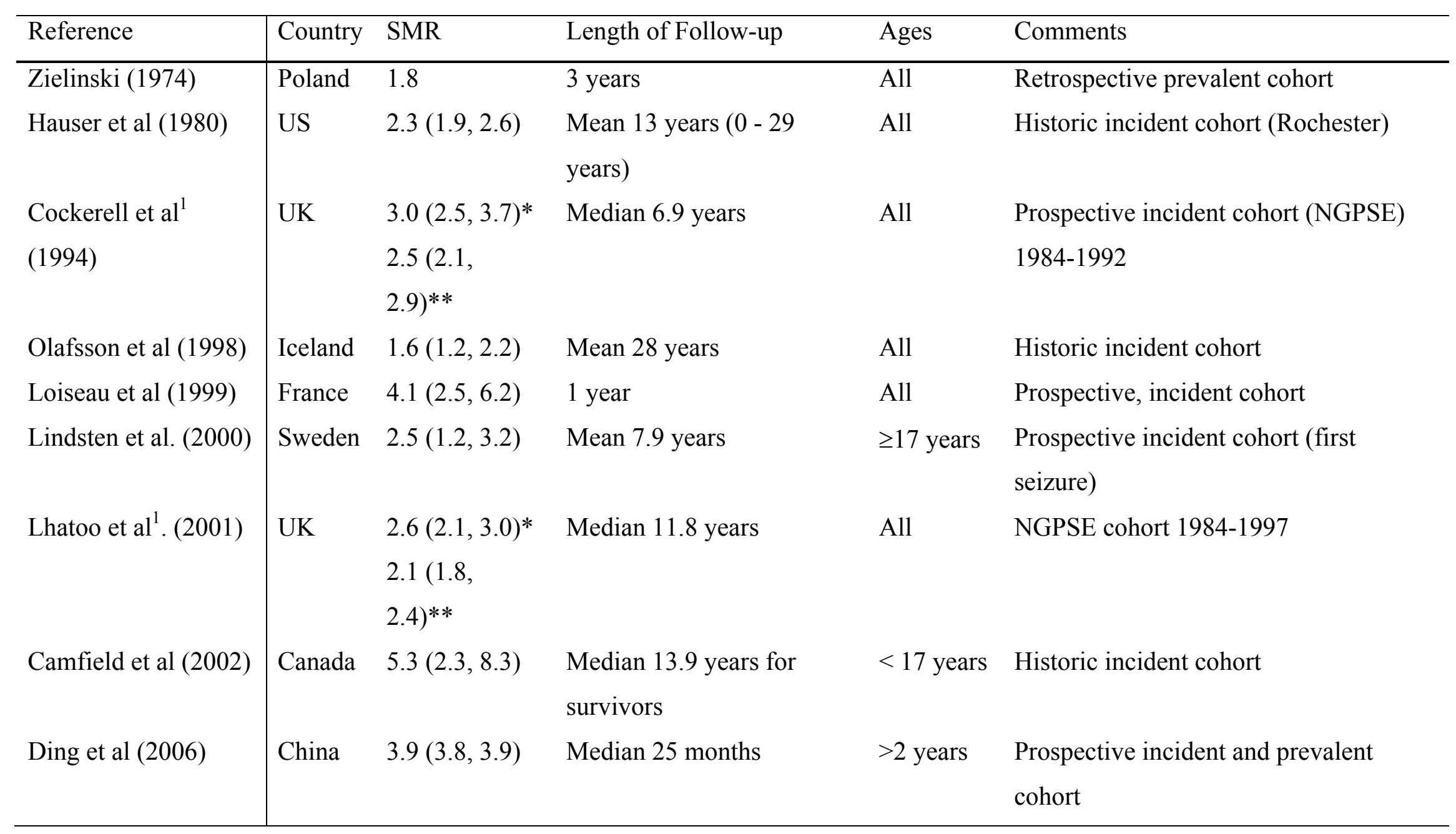

Table 8: Population studies of mortality in people with epilepsy with Standardised Mortality Ratios (with 95\% confidence intervals)

* Definite epilepsy, ** definite and possible epilepsy.

${ }^{1}$ Two papers reported mortality rates for the NGPSE cohort, after a median follow-up of 6.9 and 11.8 years respectively. 
A Swedish study identified 107 people, newly diagnosed with unprovoked epileptic seizures between 1985 and 1987, and followed them until death or until 1996 (Lindsten et al., 2000). The overall SMR was significantly increased $(2.5 ; 95 \%$ CI $1.2,3.2)$. In the first year the SMR was $7.3(95 \% \mathrm{CI} 4.4,12.1)$ and in the second year it was $3.6(95 \% \mathrm{CI} 1.6,8.1)$, following which the SMR decreased to a plateau level of between 1 and 2 for years 4-9 of follow-up (apart from year 4 at which the SMR rose slightly above 2 and year 6 at which it dropped below 1). In years 9 to 11 , however, the SMR was 5.4 (95\% CI 2.7, 11.2).

In the NGPSE the incident cohort of 792 people with definite or possible epilepsy, identified between 1984 and 1987, was followed for 14 years. The initial mortality data was reported at a median follow-up of 6.9 years, when the SMR for people with definite epilepsy was $3.0(95 \%$ CI $2.5,3.7)$ and for those with definite or possible epilepsy was 2.5 $(95 \%$ CI $2.1,2.9)$. The SMR for those with definite or possible epilepsy combined was maximum at one year since diagnosis at 5.1 (95\% CI 3.8, 6.5), decreasing to 2.5 (95\% CI $1.5,3.9)$ at four years and $1.3(95 \%$ CI $0.7,2.0)$ at greater than five years (Cockerell et al., 1994).

At a median follow-up of 11.8 years the overall SMR (definite and possible epilepsy) was $2.1(95 \%$ CI $1.8,2.4)$. The SMR decreased to $1.3(95 \%$ CI $0.9,1.7)$ at years 4 to 9 and was slightly higher at $1.6(95 \%$ CI $1.1,2.2)$ at years 9 to 14 years after diagnosis. This suggestion of a trend was more marked for those with definite epilepsy $(n=564)$, for whom the SMR was significantly elevated at all points of follow-up (Lhatoo et al., 2001). It had previously been shown in this cohort that people with epilepsy have a decreased life expectancy dependant on the severity of the epilepsy (Gaitatzis et al., 2004). 
A Canadian study followed 692 children (up to 16 years old) who developed epilepsy between 1977 and 1985, for a median of 13.9 years (range $0-22.5$ years) from the time of diagnosis (Camfield et al., 2002). The SMR from 1980 to 1989 (corresponding to 0 to 12 years since diagnosis) was 5.3 (95\% CI 2.3, 8.3) and for 1990-1999 (5 to 22 years since diagnosis) was $8.8(95 \%$ CI $4.2,13.4)$. Twenty years after the onset of seizures $6.1 \%(95 \%$ CI 3.0, 9.2) of the cohort had died.

In a retrospective Icelandic population-based study of 224 children and adults, the overall SMR after 30 years was 1.6 (95\% CI 1.2, 2.2) (Olafsson et al., 1998). This risk was most marked in those with remote symptomatic epilepsy (SMR 2.3; 95\% CI 1.4, 3.5) while there was no significantly increased risk in any age group for those with idiopathic epilepsy at any point during follow-up (overall SMR $1.3 ; 95 \%$ CI $0.8,1.9$ ). The overall risk of mortality was only elevated for the first 14 years of follow-up, returning to baseline risk thereafter. The SMR was $3.0(95 \%$ CI $1.5,5.4)$ at 0 to 4 years follow-up, 2.1 (95\% CI 1.0, $3.9)$ at 5 to 9 years, $2.0(95 \%$ CI $1.0,3.8)$ at 10 to 14 years follow-up and between 0.7 and 1.2 thereafter.

In the Mayo Clinic Linkage study, a historical cohort of all residents in Rochester, Minnesota who had a first diagnosis of epilepsy between 1935 and 1974, the SMR for the total group after 29 years follow-up was 2.3 (95\% CI 1.9, 2.6) (Hauser et al., 1980). The SMR was initially high at $3.8(95 \%$ CI $2.8,5.0)$ at 0 to 1 years follow-up, subsequently decreasing to $2.4(95 \%$ CI $1.7,3.3)$ at 2 to 4 years and SMR $2.0(95 \%$ CI $1.4,2.7)$ at 5 to 9 years. The SMR was not significantly elevated at 10 to 14 years follow-up (SMR 1.4; 95\% CI 0.8, 2.2), 15 to 19 years (SMR 1.4; 95\% CI 0.7, 2.5) and 20 to 24 years (SMR 1.8; 95\% 
CI $0.7,3.0$ ) but was again significantly raised at 25 to 29 years follow-up. (SMR 3.9; 95\% CI 1.8, 7.6)

The SMRs by broad aetiological category for these studies are shown in Table 8. The SMR is either not increased, or is slightly increased in people with idiopathic/cryptogenic epilepsy, is between 2.2 and 3.7 for those with symptomatic epilepsy, but is much higher in people with a congenital neurological deficit. Most studies did not have comparable data for specific causes of death. 


\begin{tabular}{|c|c|c|c|c|}
\hline Reference & Idiopathic/Cryptogenic & Acute Symptomatic & Remote Symptomatic & Congenital Deficit \\
\hline & $\operatorname{SMR}(95 \% \mathrm{CI})$ & SMR $(95 \%$ CI $)$. & $\operatorname{SMR}(95 \% \mathrm{CI})$. & $\operatorname{SMR}(95 \% \mathrm{CI})$. \\
\hline Hauser et al (1980)* & $1.8(1.4,2.3)$ & \multicolumn{2}{|c|}{$2.2(1.8,2.7)$} & $11(6.9,16.4)$ \\
\hline Olaffson et al (1998)** & $1.3(0.8,1.9)$ & N/A & $2.3(1.4,2.5)$ & N/A \\
\hline Lhatoo et al (2001) & $1.3(0.9,1.9)$ & $3.0(2.0,4.3)$ & $3.7(2.9,4.6)$ & $25(5.1,73.1)$ \\
\hline Lindsten et al (2000) & $1.1(0.5,2.4)$ & N/A & $3.3(2.4,4.5)$ & N/A \\
\hline Camfield et al (2002)*** & \multicolumn{3}{|c|}{$1.51(0.19,2.83)$} & $\mathrm{N} / \mathrm{A}$ \\
\hline
\end{tabular}

Table 9: Population studies of mortality in people with epilepsy with Standardised mortality ratios (with 95\% confidence intervals) for the major aetiological categories

N/A Not available

*Acute and remote symptomatic causes could not be differentiated

**14 of 42 with remote symptomatic seizures had learning disability or cerebral palsy

***Only SMRs for the whole cohort and for those without a severe neurological deficit 


\subsubsection{Hospital-based studies}

The largest hospital-based study was a Swedish study which identified 9,061 people aged 15 to 97 years who were admitted for inpatient care with a diagnosis of epilepsy between 1980 and 1989 (people with both incident and prevalent epilepsy) (Nilsson et al., 1997). Four thousand and one people in the cohort died. The overall SMR was 3.6 (95\% CI 3.5, 3.7), highest in young people but significantly elevated in all age groups. The overall SMR was compared with that in people who died more than four years after entering the cohort and thus at least four years after onset of epilepsy. The SMR in the group who died later was $3.1(95 \%$ CI 3.0, 3.3), lower than the SMR of the whole cohort but within the same range. No comparison was made of the SMRs for the group who died less than or equal to four years after entering the cohort and those who died more than four years after entering the cohort.

In a retrospective Scottish study of people attending epilepsy services between 1981 and 2001, those with newly diagnosed epilepsy $(\mathrm{N}=890)$ had a $42 \%$ increased risk of premature mortality compared with the general population (SMR 1.42; 95\% C.I 1.16, 1.72) (Mohanraj et al., 2006). People who did not respond to treatment had a greater increase in mortality (SMR 2.54; 95\% CI 1.83, 3.44) while people in remission had no increased risk (SMR $0.95 ; 95 \%$ CI $0.68,1.29)$.

In a Danish study (Henriksson et al., 1967) cited elsewhere, of 2,450 people with prevalent epilepsy aged 15 to 89 attending four epilepsy outpatient clinics from 1950 to 1963, and excluding those with acute symptomatic causes, the overall SMR for the cohort was 2.8, with males having a higher SMR (3.5) than females (2.0) (Shackleton et al., 2002). 


\subsubsection{Studies from institutions}

In a retrospective Dutch study, the mortality of all people attending an institution (both inpatients and outpatients) between 1953 and 1967 was investigated with a mean follow-up of 28 years (range 6 months to 41 years) (Shackleton et al., 1999). The overall SMR for the cohort with incident epilepsy was 3.2 (95\% C.I 2.9, 3.5). The SMR was significantly elevated soon after diagnosis (SMR 16; 95\% CI 11, 20 in the first two years, decreasing thereafter to an SMR of approximately 7.0 for the next eight years. The SMR decreased further to 3.9 and subsequently plateaued at between 2.0 and 2.4 between 15 and 34 years. The SMR was no longer significantly elevated in the last years of follow-up (years 35-41: SMR 0.9; 95\% CI 0.4, 1.4).

Two studies carried out in institutions for people with mental disorders in the 1930s and 40s were identified, one from the United States and the other from Sweden (Alstrom, 1942; Malzberg, 1934). Both these studies comprised people with LD and prevalent epilepsy, (Shackleton et al., 2002) and both reported high SMRs; 8.0 (United States) and 8.7 (Sweden).

In a study from the Chalfont Centre for Epilepsy in the UK, deaths in 2,099 people with prevalent epilepsy who were admitted between 1931 and 1971 were investigated. Between 1951 and 1977636 people died (both while at the centre and after discharge), giving an SMR of 3.0 (95\% CI 2.8, 3.3) (White et al., 1979). In another study from the Chalfont Centre, the death certificates of all patients who died between January 1980 and December 1990 were examined; there were 113 deaths giving an SMR of $1.9(95 \%$ CI 1.6, 2.3) (Klenerman et al., 1993). The deaths occurring in people still resident in the Chalfont 
Centre in five year periods from 1896-1965 were also examined (O'Donoghue and Sander, 1997). During this period, there were 416 deaths, with an overall SMR of 2.34 (95\% CI 2.12, 2.56). The SMR remained relatively stable at between 1 and 3 throughout most of the period of follow-up, with the lowest reported SMR (0.91) occurring during the earliest period of observation (1896-1900) and the highest occurring between 1916-1920 (4.41), which may in part be explained by the Spanish Flu pandemic preferentially affecting death rates in institutions. 


\subsection{Population-based mortality trends in people with epilepsy}

Mortality rates (age-adjusted mortality rates per million) per year from all causes in ten developed countries (Australia, Canada, England \& Wales, France, Germany, Italy, Japan, The Netherlands, Spain and the United States) were studied between 1979 and 1997 to determine if there were any changing patterns in neurological deaths (Pritchard et al., 2004). There were significant differences in the annual mortality rates between different countries, ranging from 6 per million in Japan to 24 per million in France but there was little variation in mortality rates year by year in each country. Males had a consistently higher mortality rate than females in all countries.

The trends in annual mortality rates (and hospital admissions) in England and Wales between 1993 and 2000 were examined using ICD-9 coding for death certificates (Bruce et al., 2004). Epilepsy was mentioned on the death certificate in about 2,000 cases and listed as the underlying cause of death in approximately 800 cases each year. Overall there were 15,990 death certificates where epilepsy was mentioned and 6,497 (40.6\%) in which it was listed as the underlying cause of death. Age-standardised annual death rates for epilepsy varied little between 1993 and 2000. Deaths were higher in males for each year with a mean of 484 deaths attributable to epilepsy each year (compared with 332 in females). Males had higher annual mortality rates than females for all age groups. In both males and females there was a sharp increase in mentions of epilepsy in the elderly.

The mortality rates (per million person-years) of people with epilepsy between 1950 and 1994 in England \& Wales and the United States were analysed (O'Callaghan et al., 2000). Trends were broadly similar for both countries with mortality consistently higher in males, although mortality rates were higher in England and Wales than in the United States. The 
mortality rate declined in both countries between birth and the age of ten, increasing sharply during teenage years and early adulthood. Rates fell gradually thereafter until the age of 70 , when they increased, with the increase in mortality in those aged greater than 70 years being more pronounced in England and Wales. There was evidence of significantly decreased mortality rates in successive birth cohorts born after 1950; this decrease occurred earlier among women (after 1905) but was more marked after 1950. Similarly the age distribution of those who died also changed between 1950 and 1994 with a two- to threefold decrease in mortality in young people, a decrease in young and middle-aged adults (with some differences between the countries) and a steep decrease in the elderly between 1950 and 1970 but an increase thereafter (O'Callaghan et al., 2000). There was little evidence of period effects. Mortality was highest during the 1950 s, decreased slightly until the 1970s and remained relatively stable thereafter in the United States, while increasing slightly in England/Wales after 1980 (O'Callaghan et al., 2000). An earlier study (Massey and Schoenberg, 1985), which compared mortality rates in 33 countries between 1967 and 1973 with the mortality rates for the same countries in the 1950s (Goldberg and Kurland, 1962), found a significant decrease in mortality rates for most countries.

A summary of these findings is discussed on Page 207. 


\section{Chapter 4: $\quad$ National General Practice Study of epilepsy (NGPSE) (Study 2)}

\subsection{Methods}

\subsubsection{Study design}

\subsubsection{Participants}

The National General Practice Study of Epilepsy (NGPSE) is a prospective cohort study, the design and methodology of which has been previously reported (Hart et al., 1989). In summary, people with newly diagnosed seizures (or possible seizures) were registered with the study between June 1984 and October 1987 by 275 general practitioners (GPs) across the UK, who were asked to notify to the study all people seen with newly suspected seizures apart from neonatal seizures.

\subsubsection{Classification}

At six months, a diagnostic review panel (consisting of two neurologists and one paediatric neurologist) reviewed all 1,195 cases registered using all contemporaneous available information, including detailed eye-witness accounts of the episodes, clinical course and investigations (EEG, Neuro-imaging and the clinical assessment of their attending hospital physician). The panel members designated people as having possible/probable epilepsy where they were undecided whether the episodes represented epileptic seizures or not, or in people in whom epilepsy was felt to be the likely diagnosis but that further clinical information was needed to confirm the diagnosis. The panel classified $564(47.2 \%)$ people 
as having definite epilepsy, $228(19.1 \%)$ as having possible epilepsy and $220(18.4 \%)$ as having febrile seizures; the remainder $(183,15.3 \%)$ were either excluded (104 with a prior diagnosis of epilepsy or neonatal seizures), or classified as not having epilepsy (79). (Those classified as not having epilepsy included those with a final diagnosis of syncope, nonepileptic attacks, vascular events, night terrors, breath-holding attacks, vertigo and migraine.)

Those classified as having definite epilepsy were subdivided broadly by aetiology into idiopathic/cryptogenic (no obvious cause identified [346]), acute symptomatic (seizures within three months of a defined risk factor [83]), remote symptomatic (seizures more than three months after a risk factor [119]), and seizures occurring in association with a neurological deficit presumed present at birth (16) (Annegers et al., 1979). Those in whom no obvious cause was identified included people with idiopathic epilepsy (IGE) and cryptogenic epilepsy (people with focal onset epilepsy in whom no structural abnormality was identified). As the NGPSE is an observational study no additional investigations were carried out beyond that which was indicated clinically. No further sub-analysis of those groups has been carried out in this thesis, an approach consistent with previous reports (Hart et al., 1989; Sander et al., 1990).

A new definition of acute symptomatic seizures has recently been proposed by the ILAE (Beghi et al., 2010) of a seizure occurring usually within seven days of an acute CNS insult. Consequently those originally classified as having acute symptomatic seizures $(\mathrm{N}=83)$ have been reclassified in line with this definition and the SMRs for the acute and remote symptomatic groups recalculated. Analyses using both the original and the new classifications are presented in the mortality section (4.2.1). 
The original classifications of definite epilepsy, possible/probable epilepsy and febrile seizures were maintained throughout the analysis in the thesis.

\subsubsection{Initial follow-up}

People with definite and possible epilepsy were initially followed up at 6 months after registration and then yearly thereafter. Follow-up was by means of postal questionnaires completed by the person's GP, detailing seizure frequency, seizure recurrence, seizure semiology, hospital follow-up and AED treatment. Follow-up for the cohort was yearly up to 1997. A further follow-up was performed in 2001 (unpublished) but was not completed due to changes in Data Protection Regulations. Children with febrile seizures were initially followed for two years after the close of recruitment with one further follow-up in 1998 (MacDonald et al., 1999).

The results presented in the thesis represent the most complete attempted follow-up of the NGPSE cohort since the early years of the study.

\subsubsection{Ethical approval}

The NGPSE was originally approved by the local ethics committee of the National Hospital of Neurology and Neurosurgery and the Royal College of General Practitioners. In light of the changes to the data protection legislation in 2001, the NGPSE research group were informed that we would no longer be able to follow the cohort up without consent. When the decision to attempt a further follow-up of the NGPSE cohort was reached, it was therefore necessary made a new application to the National Research Ethics Committee. Ethics approval was obtained for the study in November 2007 (REC Reference: 07/H0720/160), the application being subject to four further (one substantive) amendments with final ethical approval being granted in January 2009. 


\subsubsection{Data protection and the implications for the NGPSE}

When the NGPSE was set up, like all other observational epidemiological studies with no direct patient contact, individual patient consent was not necessary for registration with the study, with the issue of obtaining individual patient consent left to the discretion of the notifying general practitioners. Following the enactment of the Data Protection Act 1998 and the Health \& Social Care Act 2001 (www.legislation.gov.uk/ukpga/1998/29/contents; www.legislation.gov.uk/ukpga/2001/15/content), GPs would no longer be able to release identifiable patient information (necessary for the study) without direct patient consent to do so without being in breach of confidentiality and GMC guidance. There is, however, a provision (Section 60 exemption of the Health \& Social Care Act 2001) which allows for the disclosure of patient information without explicit consent, when it is impractical to obtain patient consent or when an important public interest is at stake, (as in the case of cancer registries which were one of the primary reasons for the provision of such an exemption). We believed that the necessity of obtaining patient consent for the release of information would inevitably result in a significant reduction in case ascertainment, and also that it was impractical to ask GPs to obtain patient consent; thus the only practical way for the study to continue was with Section 60 support. It was, however, recognised that such a request, if successful, would likely only be granted on a one-off basis and would therefore preclude any further follow-up of the cohort and the inevitable end of the study.

\subsubsection{PIAG and the NGPSE}

Following the granting of ethical approval for the study, an application for Section 60 exemption for the study was made to the Department of Health Patient Information Advisory Group (PIAG) in December 2007 with the application being considered at the 
PIAG meeting in February 2008. This application was accompanied by a strong letter of support for the study by the UCLH Caldicott Guardian. Prior to the PIAG meeting (which occur every two months) there was extensive communication (both via email and telephone) with the PIAG policy support management clarifying diverse aspects of the study and the necessity for such an application. The application for Section 60 exemption was considered at the PIAG meeting of the $5^{\text {th }}$ February 2008 and was not successful. The primary reasons, as stated in the outcome letter, which the Group gave for not granting Section 60 exemption for the study were expressed as follows:

It is a principle of the Group that long term retention of identifiable information is only done with consent. Whereas members recognise that obtaining patient consent for historical studies can be difficult, the Group noted that you are intending to contact patient's GPs and this is an ideal opportunity to ask them to publicise the study and to attempt to obtain consent. As this appears to be a reasonable alternative to Section 60 support, members felt that it would be inappropriate to offer Section 60 support in this instance. However, if it can be shown that seeking consent via GPs is impracticable then PIAG would welcome evidence of this.

Upon receipt of the decision letter, we informed the PIAG support management of our intention to appeal against this decision. There followed further intense communication between the NGPSE Research team and the PIAG Support Management culminating in a meeting with the head of the PIAG secretariat (with Professor Sander and Aidan Neligan) and a formal request to attend the next PIAG meeting on $14^{\text {th }}$ April 2008. This was granted and was attended by Professor Shorvon and Aidan Neligan. At the meeting the Group initially argued that permission to ask GPs whether the patient still had epilepsy or not 
would be sufficient for the study. We argued that such a provision would both be inappropriate (in the cases of febrile seizures and possible epilepsy) and meaningless (as a diagnosis of epilepsy is subject to different interpretations by different clinicians).

Ultimately we were granted leave to resubmit the application for consideration at the next meeting following clarification of several points of contention. It was agreed that the application would be for a one-time approval and that the study would end thereafter. The application was reconsidered at the PIAG meeting of the $25^{\text {th }}$ June and the study was granted one-off Section 251 support (Section 60 of the Health and Social Care Act 2001 was re-enacted under Section 251 of the NHS Act 2006) under the following conditions (with the questionnaire being amended accordingly [see Appendix]).

The first two questions in the questionnaire were changed to the following:

1) Has the patient had any seizures in the past five years? (this is the point at which patients are believed to be in long-term remission)

2) Has the patient taken any medication for epilepsy in the past five years?

If the answer to both of these questions was no then the GPs had permission to complete the questionnaire without patient consent on this single occasion. If, however, the answers to either of these questions was yes then patient consent would be required to complete the questionnaire. (The GPs could, however, answer the first two questions without patient consent and therefore return the questionnaire partially completed). The rationale for such a decision was that if a patient had had seizures or was on medication for seizures in the previous five years, then the patient would clearly be aware of a diagnosis of epilepsy or seizures and would therefore be in a position to grant consent for inclusion in an epilepsy study. These conditions were accepted and formal notification of the awarding of Section 
251 support for the study was received at the end of August 2008 with an initial duration of one year. This approval was formally extended by another year in September 2009.

\subsubsection{The Office of National Statistics (ONS) and the NGPSE}

The whereabouts of all people in the NGPSE cohort was tracked by the Information Centre NHS (formerly the ONS and previously the NHS Central Registry). Given that the last attempted follow-up was over eight years previously, it was believed that a significant number of people may have moved. A request was made to be notified of the location of the current GP of each person in the study (Members and Posting application). Such a request required approvals from the ethics committee of the Central Register as well the Ethics and Confidentiality Committee of the National Information Governance Board for Health and Social Care (NIGB; formerly PIAG) which was formerly granted in March 2009.

The locations of GPs for all people in the study were given in April 2009, listed by area with the contact details for the Patient Data Management team for each area, to whom a request for the details of the GP practices could then be made.

\subsubsection{Research \& Development Departments}

In order to carry out research in an individual Primary Care Trust (PCT) (i.e. sending the follow-up questionnaire to a GP practice in that area), approval from the PCT Research \& Development Department (R\&D department) is required. Applications to individual (\& consortiums of) R\&D departments started in late February 2009 continuing up to September 2009 with further applications made as required when the questionnaires were being sent out. There was initial confusion as to the exact number of PCTs for which 
approval would be required as the list of areas supplied by the Information Centre NHS did not exactly correspond to the geographical areas of the PCTs (for example the area designated "East Riding” contained practices in East Riding PCT and Hull City PCT), necessitating more PCT approvals than originally anticipated based on the list of "PCTs" provided by the Central Register. In total approvals were required from 120 PCTs (including seven in Scotland and three Health Authorities in Wales). In addition to obtaining approvals from several PCTs which, in the end, did not contain any people in the NGPSE cohort we were also required to obtain individual PCT approvals for one person in the study in several instances. One PCT initially did not grant approval for the study but did so upon appeal.

This process of applications to PCT R\&D departments for approval was greatly facilitated by the fact that we were successful in getting the study adopted onto the National Institute for Health Research (NIHR) Comprehensive Research Network (CRN) and the Primary Care Research Network (PCRN) portfolios. This had the added bonus that the study was now eligible for Service Support Costs and therefore GP practices (in England) could be paid for participating in the study.

\subsubsection{Scotland and the NGPSE}

In contrast to England \& Wales, in Scotland there is no provision comparable to Section 251 Support to allow long-term epidemiological cohort studies such as the NGPSE to proceed without consent. It was therefore anticipated that consent would be required from all people in the NGPSE cohort in Scotland. (The issue was discussed at length with the Privacy Advisory Committee (PAC) which confirmed this view). Accordingly separate 
research documents were drawn up for Scotland to reflect the different ethical situation. Given this requirement for consent from all people in the study in Scotland, it was our original intention to phone all practices to discuss and stress the importance of the study with each GP (in Scotland) prior to sending out the questionnaire. This was not possible as it was the contention of the PAC and the General Register Office that the study did not have the authorisation to have GP details. Therefore the questionnaires would need to be forwarded to GPs through the intermediary of the NHS Central Register (NHSCR) Scotland (who in turn sent the envelopes containing the questionnaires to the appropriate Practitioner Services Divisions Office who then forwarded the information to the relevant GPs).

\subsubsection{Current follow-up}

The current follow-up questionnaires were sent out between October 2009 and April 2010. In some cases the questionnaire was sent out a second time when the original correspondence had been misplaced. Where possible, all practices from whom a response had not been received after three months, were contacted by telephone (initially via the practice manager) and the questionnaire completed over the phone with the GP in several instances. Such an approach was not possible in Scotland.

\subsubsection{Mortality analysis}

All people in the study were flagged at the UK NHS Information Centre who notified the study group of all deaths of people in the study and the death certificates were obtained where available, ensuring complete case ascertainment in the UK. This was made possible because the study had previously obtained Section 60 Support for the study in relation to 
notification of deaths in the study as well as approval from the PAC, National Services Scotland and the General Register Office Scotland.

The principal cause of death was obtained from the death certificates (which were available in all but three cases) and allocated ICD codes following deliberation by the study review panel. Mortality data from the NGPSE cohort has previously been reported at a median of 6.9 years from registration with a total of 161 observed deaths and 7,500 person-years of observation (Cockerell et al., 1994), and at a median of 11.8 years with 214 deaths and 11,400 person-years (Lhatoo et al., 2001).

\subsubsection{Mortality statistical analysis: statistical analysis}

Expected numbers of deaths were estimated using age ( 5 or 10 year bands), sex and calendar year-specific death rates in England and Wales. Data were obtained from the Registrar General's mortality statistics (up to 1997), the CD $20^{\text {th }}$ century mortality (1998) and the website of the Information Centre NHS (1999 onwards) (www.statistics.gov.uk/STATBASE; Office of National Statistics, 2003 Twentieth century mortality [CD-ROM]). SMRs with 95\% CIs were calculated (for the whole cohort and for those with definite epilepsy, possible epilepsy, febrile seizures and for subgroups of those with definite epilepsy) using the person-years method with SMRs and 95\% CIs based on the Poisson distribution, and two-tailed significance tests. SMRs for specific causes were also calculated for those with definite and possible epilepsy.

SMRs were calculated at various follow-up intervals and for different age groups in the group with definite and possible epilepsy. Analysis was carried out in Stata (version 10; Statacorp LP, Texas) with participants followed up from registration in the study until death or, if alive, to $30^{\text {th }}$ April 2009. 
4.1.3.3 Febrile seizures statistical analysis

The age-specific incidence rates reported in the British National Child Development Study cohort (Kurtz et al., 1998) were used to calculate the number of children with febrile seizures expected to develop epilepsy (with ages 24 to 30 years extrapolated from age 23 years). Standardised incidence rates (SIRs) with 95\% CIs were calculated in five-year age bands using the person-years method with the SIRs and 95\% CIs based on the Poisson distribution and two-tailed significance tests. 


\subsection{Results}

\subsubsection{The long-term risk of mortality in people with epilepsy}

Median follow-up was 22.8 years, and 19,144 person-years of follow-up in those with definite or probable epilepsy or febrile seizures was achieved. There were 301 deaths in the cohort. Thorough re-examination of all the source data files held by the NGPSE research group (all previous completed follow-up questionnaires, death certificates) resulted in some minor changes in demographic data and allocation of cause of death.

The overall SMR was increased for those with definite epilepsy (SMR 2.55) and those with possible epilepsy (SMR 1.57) (Table 10). 


\begin{tabular}{|c|c|c|c|c|c|c|c|c|c|c|}
\hline \multirow[t]{3}{*}{ Group } & \multirow{3}{*}{$\begin{array}{l}\text { Number } \\
\text { at risk }\end{array}$} & \multicolumn{9}{|c|}{ Number of deaths } \\
\hline & & \multicolumn{2}{|c|}{ Males } & \multicolumn{2}{|c|}{ Females } & \multicolumn{2}{|l|}{ Total } & \multirow[b]{2}{*}{ SMR } & \multirow[b]{2}{*}{$95 \% \mathrm{CI}$} & \multirow[b]{2}{*}{$\mathrm{p}$} \\
\hline & & Obs & Exp & Obs & Exp & Obs & Exp & & & \\
\hline $\begin{array}{l}\text { Definite } \\
\text { epilepsy }\end{array}$ & 564 & 122 & 50.7 & 103 & 37.6 & 225 & 88.2 & 2.55 & 2.24 to 2.91 & $<0.001$ \\
\hline $\begin{array}{l}\text { Possible } \\
\text { epilepsy }\end{array}$ & 228 & 33 & 17.9 & 42 & 29.9 & 75 & 47.8 & 1.57 & 1.25 to 1.97 & $<0.001$ \\
\hline Subtotal & 792 & 155 & 68.5 & 145 & 67.5 & 300 & 136.0 & 2.21 & 1.97 to 2.47 & $<0.001$ \\
\hline $\begin{array}{l}\text { Not } \\
\text { epilepsy }\end{array}$ & 79 & 10 & 5.5 & 15 & 12.6 & 25 & 18.0 & 1.39 & 0.93 to 2.05 & 0.072 \\
\hline $\begin{array}{l}\text { Febrile } \\
\text { seizures }\end{array}$ & 220 & 1 & 2.1 & 0 & 0.9 & 1 & 3.0 & 0.34 & 0.05 to 2.39 & 0.950 \\
\hline Total & 1091 & 166 & 76.1 & 160 & 80.9 & 326 & 157.0 & 2.08 & 1.86 to 2.31 & $<0.001$ \\
\hline
\end{tabular}

Table 10: All-cause mortality for Whole Cohort and Specific Subgroups

Obs $=$ observed, Exp $=$ expected 
One person with a history of febrile seizures (three febrile seizures as a child with no further seizures) died by suicide.

Mortality within different age groups is summarised in Figure 1.

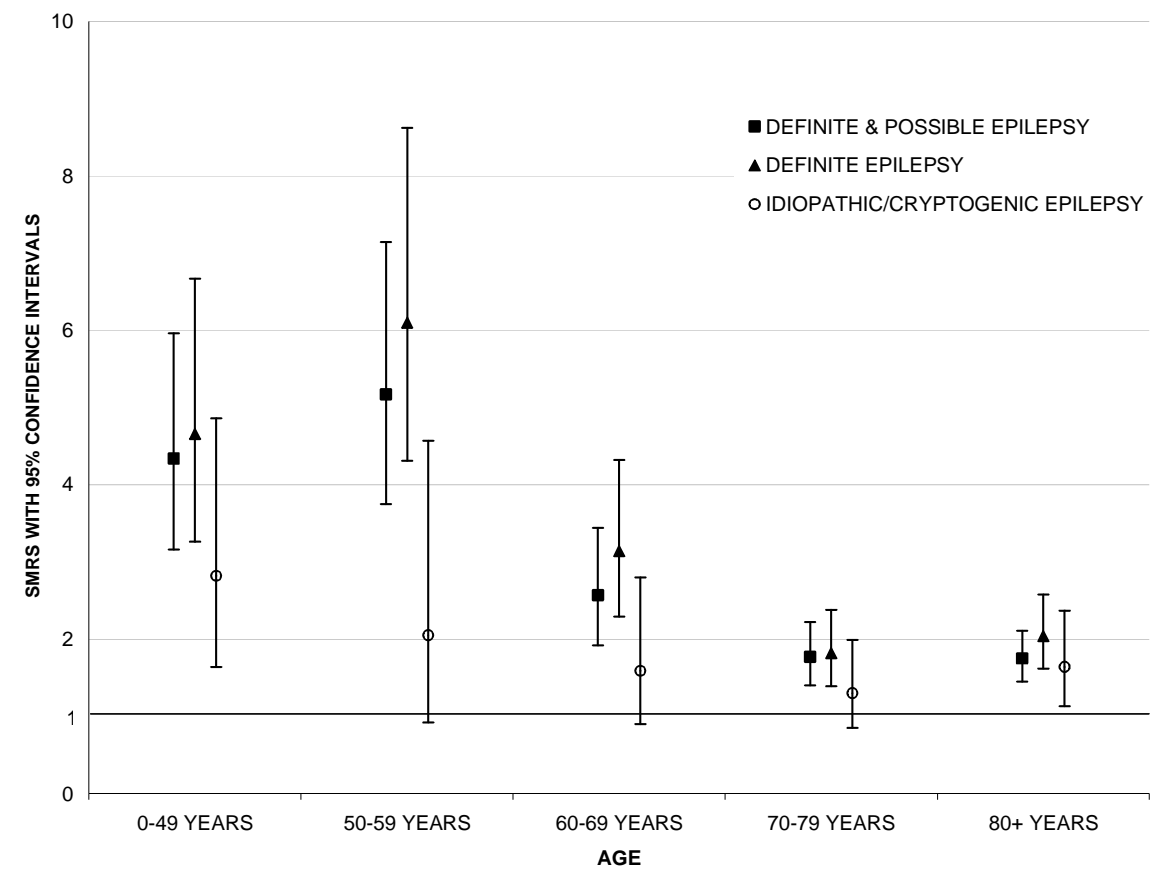

Figure 1: All cause mortality by age-group during follow-up in the combined group with definite and possible epilepsy, people with definite epilepsy, and the subgroup with idiopathic epilepsy

Mortality ratios were significantly raised for all age groups in those with definite epilepsy and the combined groups of definite and possible epilepsy. For people with definite epilepsy, the overall SMR for the different aetiologies (idiopathic/cryptogenic, acute and remote symptomatic and congenital deficit) were significantly elevated in all four groups (Table 11). 


\begin{tabular}{llllll}
\hline \multicolumn{1}{c}{ Cause } & Number & \multicolumn{3}{c}{ Number of deaths } \\
& at risk & Obs & Exp & SMR & 95\% CI \\
& & & & & \\
\hline Idiopathic/cryptogenic & 346 & 80 & 48.4 & 1.66 & 1.33 to 2.06 \\
Remote symptomatic & 119 & 98 & 26.2 & 3.73 & 3.06 to 4.55 \\
Remote symptomatic & 142 & 112 & 30.5 & 3.68 & 3.05 to 4.42 \\
(Revised 2010) & & & & & \\
Acute symptomatic & 83 & 43 & 13.4 & 3.20 & 2.38 to 4.32 \\
Acute symptomatic & 60 & 29 & 9.19 & 3.16 & 2.19 to 4.54 \\
(Revised 2010) & & & & & \\
Congenital deficit & 16 & 4 & 0.2 & 18.64 & 6.99 to 49.65 \\
\hline
\end{tabular}

Table 11: All-cause mortality in people with definite epilepsy according to aetiology

Obs. $=$ observed, Exp. $=$ expected

The SMR remained elevated for people with acute symptomatic seizures who had survived the first year (SMR 2.5, 95\% CI 1.7 to 3.5). Mortality over time for people with definite epilepsy, definite and possible epilepsy and people with idiopathic epilepsy is summarised in Figure 2. 


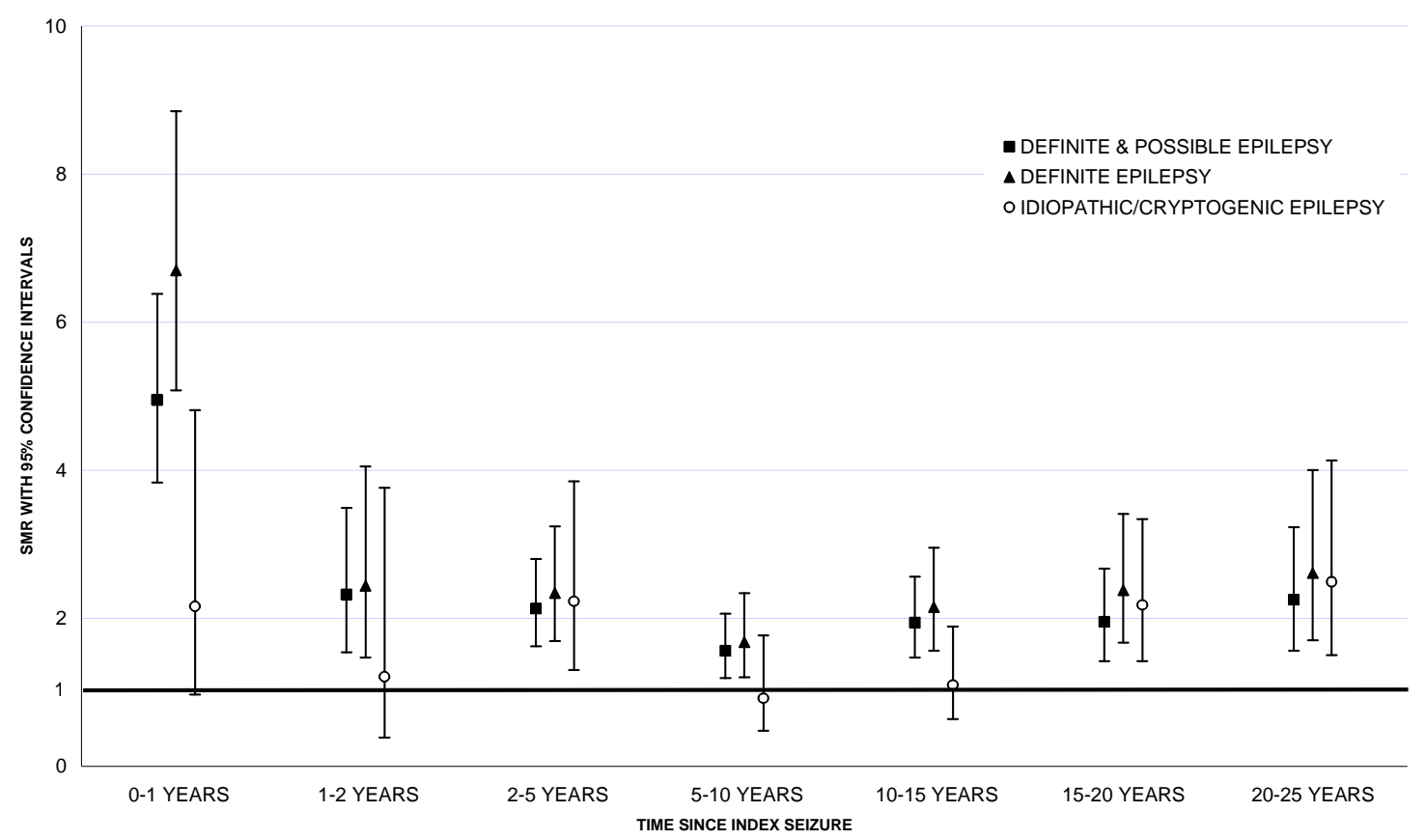

Figure 2: Mortality by years of follow-up from index seizure in the combined group with definite and possible epilepsy, people with definite epilepsy, and the subgroup with idiopathic epilepsy

Current cause-specific SMRs and those previously reported for the first seven and fourteen years are listed in Table 12. 


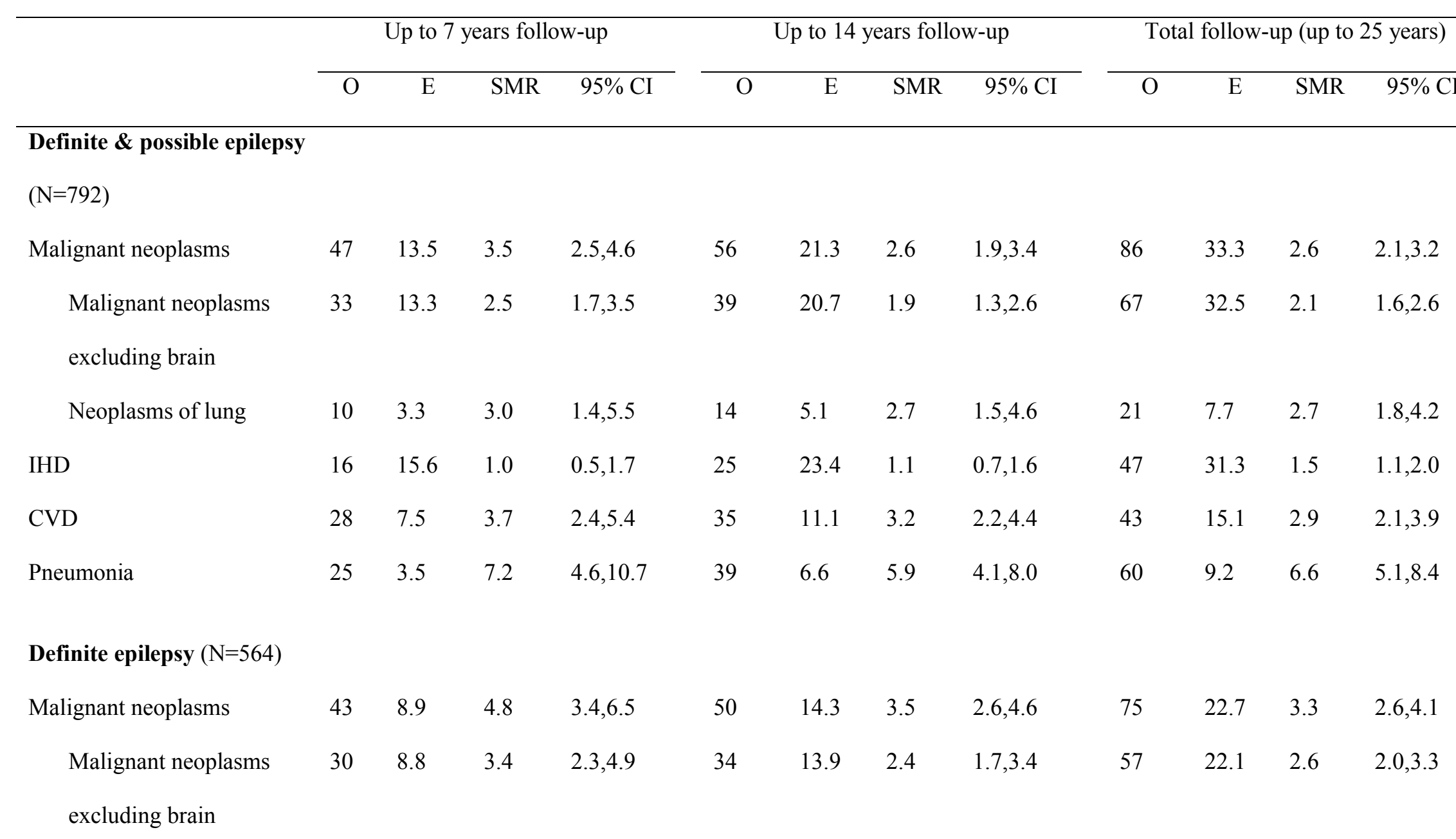




\begin{tabular}{|c|c|c|c|c|c|c|c|c|c|c|c|c|}
\hline Neoplasms of lung & 10 & 2.3 & 4.3 & $2.0,7.9$ & 12 & 3.6 & 3.3 & $1.7,5.8$ & 18 & 5.5 & 3.3 & $2.1,5.2$ \\
\hline IHD & 10 & 9.9 & 1.0 & $0.4,1.9$ & 16 & 15.1 & 1.1 & $0.6,1.7$ & 30 & 20.9 & 1.4 & $1.0,2.1$ \\
\hline CVD & 19 & 5.0 & 4.4 & $2.6,6.8$ & 24 & 6.5 & 3.7 & $2.3,5.5$ & 29 & 9.3 & 3.1 & $2.2,4.5$ \\
\hline Pneumonia & 18 & 1.9 & 9.6 & $5.6,15.1$ & 27 & 3.7 & 7.3 & $4.8,10.6$ & 44 & 5.6 & 7.9 & $5.8,10.6$ \\
\hline
\end{tabular}

\section{Idiopathic/cryptogenic}

epilepsy $(\mathrm{N}=346)$

Malignant neoplasm

$\begin{array}{llll}8 & 3.9 & 2.0 & 0.8,4.0\end{array}$

7.0

$\begin{array}{ll}7.0 & 1.3 \\ 6.8 & 0.9\end{array}$

$0.6,2.5$

23

$\begin{array}{lll}13.0 & 1.8 & 1.2,2.7\end{array}$

Malignant neoplasms

$\begin{array}{ll}5 & 3.8\end{array}$

$0.4,3.0$

6

6.8

$0.3,1.9$

18

$12.7 \quad 1.4 \quad 0.9,2.3$

excluding brain

\begin{tabular}{|c|c|c|c|c|c|c|c|c|c|c|c|c|}
\hline Neoplasms of lung & 0 & 1.0 & 0 & $0.0,3.6$ & 1 & 1.8 & 0.6 & $0.0,3.2$ & 4 & 3.1 & 1.3 & $0.5,3.5$ \\
\hline IHD & 1 & 3.9 & 0.3 & $0.0,1.4$ & 4 & 6.7 & 0.6 & $0.1,1.5$ & 15 & 11.0 & 1.4 & $0.8,2.3$ \\
\hline VD & 1 & 1.5 & 0.6 & $0.0,3.6$ & 3 & 2.7 & 1.1 & $0.2,3.3$ & 6 & 4.8 & 1.2 & $0.6,2.8$ \\
\hline eumonia & 6 & 0.6 & 10.1 & $3.6,21.8$ & 8 & 1.5 & 5.4 & $2.3,10.7$ & 20 & 3.0 & 6.7 & $4.3,10.4$ \\
\hline
\end{tabular}


Table 12: Cause-specific SMRs in the combined group with definite and possible epilepsy, people with definite epilepsy, and the subgroup with idiopathic epilepsy. Not all causes of death are included in the table.

*Up to 7 years follow-up and up to 14 years follow-up taken from Cockerell et al., 1994 and Lhatoo et al., 2001.

IHD; ischaemic heart disease: CVD cerebrovascular disease 
The 5 year SMRs for the major causes of mortality for those with definite or possible epilepsy are listed in Table 13.

\begin{tabular}{|c|c|c|c|c|c|c|}
\hline & & $\mathrm{SM}$ & with $95 \% \mathrm{Cc}$ & nfidence Inte & rvals & \\
\hline & $\begin{array}{l}\text { Malignant } \\
\text { neoplasms }\end{array}$ & $\begin{array}{l}\text { Malignant } \\
\text { neoplasms } \\
\text { excluding } \\
\text { brain }\end{array}$ & $\begin{array}{l}\text { Neoplasms } \\
\text { of lung }\end{array}$ & IHD & CVD & Pneumonia \\
\hline Years & $4.44(3.3$ & $3.11(2.2$ & 4.69 (2.7, & $1.35(0.8$ & 4.21 & $10.23(6.9$ \\
\hline $0-5$ & $6.0)$ & 4.4) & 8.3) & 2.2) & $(2.8,6.3)$ & 15.1) \\
\hline Years & $1.43(0.8$ & $1.35(0.7$ & $1.11(0.3$ & $1.13(0.6$ & 2.50 & $3.22(1.6$ \\
\hline $5-10$ & 2.6) & 2.5) & 4.4) & 2.2) & $(1.3,4.8)$ & 6.4) \\
\hline Years & 1.90 & $1.46(0.8$ & $2.14(0.7$ & 1.79 & 2.28 & $4.51(2.5$ \\
\hline $10-15$ & 3.4) & 2.8) & $6.6)$ & $(0.96,3.3)$ & $(1.0,5.1)$ & $8.1)$ \\
\hline Years & $1.81(0.98$ & $1.67(0.9$, & $1.67(0.4$ & $1.35(0.6$ & 1.04 & $8.32(4.3$ \\
\hline $15-20$ & 3.4) & 3.2) & 6.7) & $3.2)$ & $(0.3,4.1)$ & $16.0)$ \\
\hline Years & 2.19 & 2.25 & $2.55(0.6$ & $3.34(1.6$ & 1.67 & 9.88 (4.7, \\
\hline $20-25$ & 4.4) & 4.5) & 10.2) & $7.0)$ & $(0.4,6.7)$ & 20.7) \\
\hline
\end{tabular}

Table 13: Selected causes of death (with SMRs and 95\% Confidence Intervals) for people with definite or possible epilepsy

There were only seven epilepsy-related deaths in the cohort, three from status epilepticus, three from accidents and drowning, and one from possible SUDEP (Lhatoo 
et al., 1999). Epilepsy was listed as a contributory factor in 11 deaths. One further person in the cohort died in March 2009 (apparently as a result of murder), but as this death is still being officially investigated and has therefore not been formally notified to the study group by means of the death certificate, it is not included in this analysis. 


\subsubsection{Follow-up of seizure outcome}

Follow-up was commenced in October 2009 and was completed at the end of July 2010. As of October 2009, of the 1,012 people registered in the initial cohort, 304 were known to have died. An additional two people had previously indicated that they did not wish to participate further in the study and so further follow-up information was not attempted.

Consequently information was sought from the ONS on the GP details of 704 people in the study, which subsequently supplied us with the details of the local health authority of the GPs whereabouts for 659 people in the study. Of the 45 people on whom no further information was available, it was known that nine had previously emigrated.

The local health authorities provided us with the current GP details for 612 people, with a further nine people not having a registered GP. In Scotland, we did not have the authority, because of issues with consent and confidentiality, to access the GP details for the 38 people in the study resident there. Follow-up questionnaires were therefore sent out directly to GP practices on 612 people in England (607) and Wales (5). In Scotland, questionnaires on 38 people were forwarded to GPs through the intermediary of the NHS Central Register (NHSCR) Scotland (who in turn sent the envelopes containing the questionnaires to the appropriate Practitioner Services Divisions Office who in turn forwarded the information to the relevant GPs). We were subsequently informed that a further 21 people were no longer registered with the indicated GP practice and no further information on their current whereabouts was available.

In total follow-up information was potentially available on 629 people in the cohort. Two people refused consent to participate further in the study while seven GPs (11 
people) refused outright to help with the study, returning the questionnaires

unanswered. One person died after signing the consent form while one further person immigrated (to Sweden) during the follow-up period. In addition, we received two signed consent forms from two people (one in England, one in Scotland) without receiving the completed questionnaire from their GP.

By the end of July 2010, 552 responses (partial or complete) had been received, of which 540 (91.4\% of the potential number) concerned people in England, 10 (26.3\%) people in Scotland and $3(60 \%)$ people in Wales. The basic data is summarised in Table 14.

Thorough re-examination of all the source data files held by the NGPSE research group (all previous completed follow-up questionnaires) resulted in some minor changes in demographic data and seizure frequency.

\begin{tabular}{lccc}
\hline & Febrile seizures & Definite & Possible \\
& & epilepsy & epilepsy \\
\hline Total & 220 & 564 & 228 \\
Still alive & 219 & 334 & 152 \\
Follow-up information & $181(82.6 \%)$ & $253(75.4 \%)$ & $119(78.3 \%)$ \\
Seizure free in the last 5 years & $175(96.7 \%)$ & $201(80.6 \%)$ & $103(86.6 \%)$ \\
Seizure free and off AEDs & $172(95.0 \%)$ & $145(58.3 \%)$ & $90(75.6 \%)$ \\
\hline
\end{tabular}

Table 14 Basic follow-up data from the NGPSE cohort 2009-10 


\subsubsection{The long-term prognosis of people with febrile seizures}

Of the original 220 children $(60.5 \%$ male $)$ with febrile seizures originally registered with the study, 219 were still alive in October 2009. Of these 219, follow-up information was obtained in 2009-10 on 181 (82.6\%), with a mean follow-up for the whole cohort of 21.6 (SD 6.0) years (median follow-up 24 years, range 0.6 to 25.9 years). In $189(86 \%)$, the index febrile seizure was the first seizure while $26(11.8 \%)$ had a previous single febrile seizure and five $(2.3 \%)$ had two prior febrile seizures. Over two thirds $(148 ; 67.3 \%)$ had no further seizures after the index febrile seizure while 57 (25.9\%) had 1-5 further seizures during follow-up, four had 6-10 further seizures, one had 11-15 further seizures and nine (4.1\%) had 20 or more seizures and in one the number of seizures was not known.

Five years after the index febrile seizure 212 people (96.4\%) were still being followed. Of these, $144(67.9 \%)$ had had no further seizures during those five years while 58 (27.4\%) had 1-5 seizures in that time. Three had 6-10 seizures, one had 11-15 seizures and six had more than 20 seizures.

After ten years, follow-up was achieved in 211 (95.9\%). Of these $201(95.3 \%)$ had had no seizures in the preceding five years while four had had 1-5 epileptic seizures and the remaining six had many $(>20)$ seizures.

Of the $181(82.6 \%)$ with follow-up after more than 20 years, $175(96.7 \%)$ were seizure free in the preceding five years while $171(94.5 \%)$ were seizure free and off AEDs. In those who had any seizure recurrence, the mean time to recurrence from index seizure was 1.1 years (SD 2.1 years, median 0.6 years, range one day to 16 years).

For children in whom the index febrile seizure was not the first seizure, the risk of recurrence of febrile seizures was slightly higher (HR 1.76, 95\% CI 0.98, 3.2) (Figure 3) 


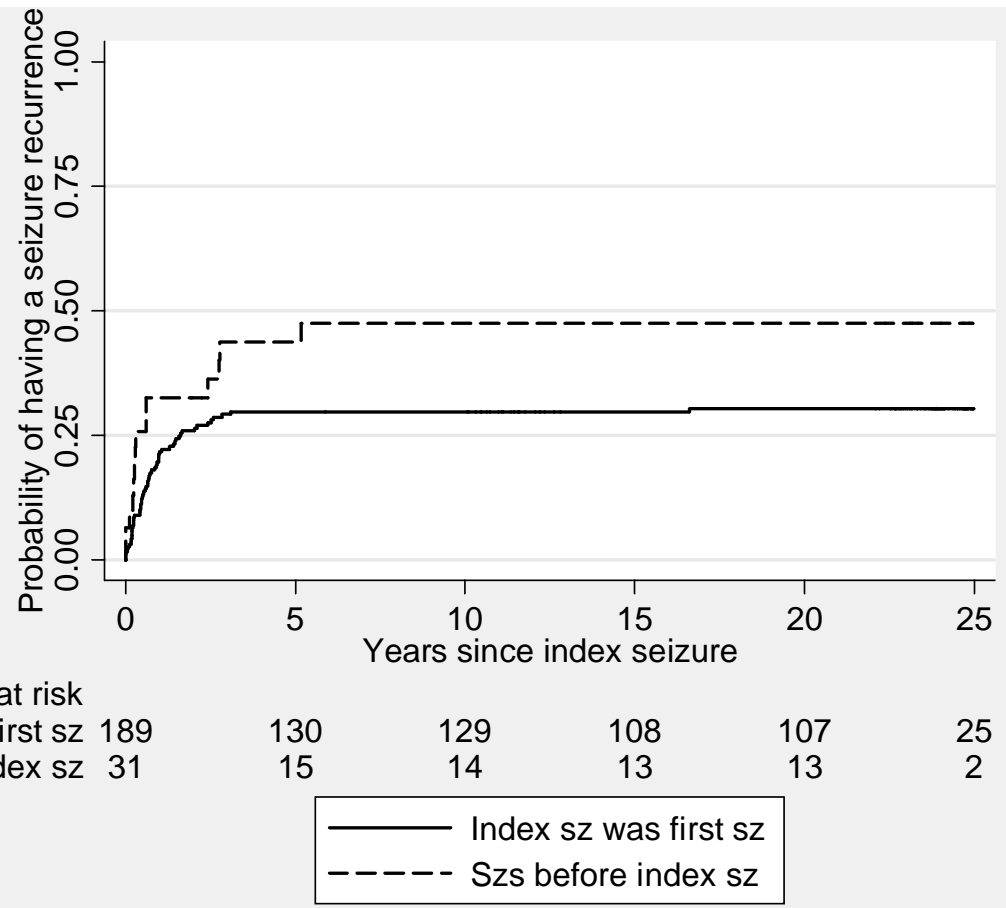

Figure 3: Cumulative risk of febrile seizure recurrence in relation to the index seizure 


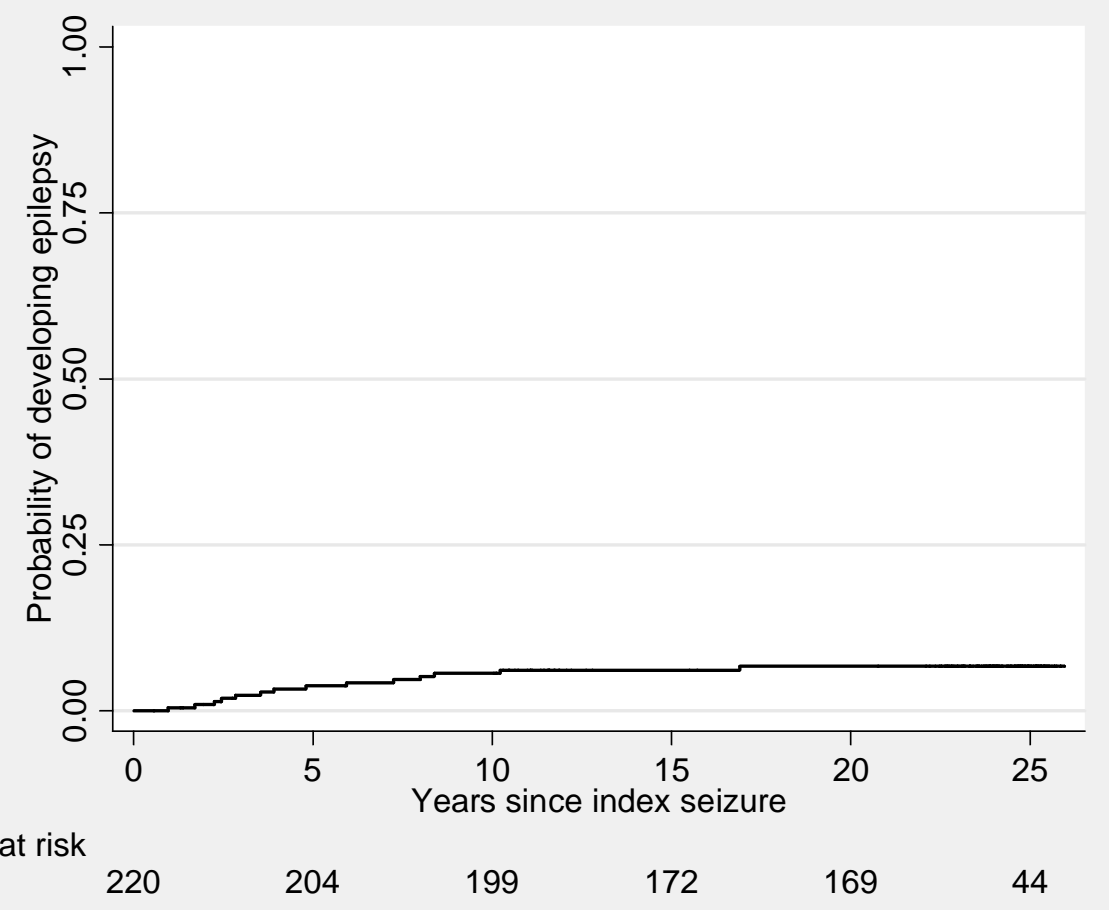

Figure 4: Time to develop epilepsy in people with febrile seizures

In total $17(7.7 \%)$ had afebrile seizures of whom $14(6.4 \%)$ had two or more afebrile seizures (epilepsy) (summarised in Table 15). The mean time to second afebrile seizure was 5.7 (SD 4.3) years (median 4.4 years; range 1.0-16.9 years) (figure 4). In the 14 people who developed epilepsy, long-term follow-up was achieved in 13 (92.9\%). At 20 years after the index febrile seizure, the estimated percentage who had developed epilepsy was $6.7 \%(95 \%$ CI $4,11 \%)$. 


\begin{tabular}{lll}
\hline Follow-up 2009-2010 & N & \multicolumn{1}{c}{ Epilepsy syndromes } \\
Seizure-free, 5 years off AEDs & 5 & CPE (3), probable CPE (1), SPE (1). \\
Seizure-free for 5 years, taking AEDs & 2 & IGE (1), CPE (1) \\
Seizure free less than 5 years, off AEDs & 1 & Probable CPE (1) \\
Ongoing seizures, taking AEDs & 5 & Nocturnal generalised seizures* (1), \\
Last follow-up 1997 & & IGE (2 - one JME), SPE (2) \\
Active epilepsy, off AEDs & & \\
& 1 & IGE (JAE)
\end{tabular}

Table 15: Outcome of people with febrile seizures who developed epilepsy $(\mathrm{N}=14)$

Information on epilepsy syndrome based on limited information in people with few seizures.

*insufficient information to allow syndromic classification Legend: $\mathrm{CPE}=$ cryptogenic partial epilepsy, $\mathrm{SPE}=$ symptomatic partial epilepsy, $\mathrm{IGE}=$ idiopathic generalised epilepsy, JME=juvenile myoclonic epilepsy, JAE=juvenile absence epilepsy

Overall the risk of developing epilepsy in the cohort was almost ten times that of the general population (Standardised Incidence Rate [SIR] 9.7, 95\% CI 5.7, 16.4). The SIR for epilepsy in the cohort in the 0-5 year age group was $14.2(95 \% \mathrm{CI} 5.9,34.2)$ and $16.3(95 \%$ CI $7.3,36.2)$ in the $5-10$ year age group. The SIR was $7.2(95 \%$ CI $1.8,28.7)$ in the 10-15 year age group and was no longer significant in the 15-20 year age group (SIR 4.5, 95\% CI 0.6, 32.0). 


\subsubsection{The long-term prognosis of people with epilepsy}

In the original cohort 792 were classified as having definite $(n=564)$ or possible $(n=228)$ epileptic seizures, of whom 486 (334 (59.2\%) with definite epilepsy) were still alive in October 2009 when the current follow-up was commenced. In 263 (46.6\%) of those with definite epilepsy and $113(49.6 \%)$ of those with possible epilepsy, the index seizure was the first seizure. In total, long-term follow-up information was available in $372(76.3 \%) ; 253(75.4 \%)$ of those with definite epilepsy who were still alive and 119 (78.3\%) of those with possible epilepsy. The mean duration of follow-up was 15.9 years (SD 8.9; median 16.6 years, range 0 to 25.9 years) for those with definite epilepsy and 16.9 years (SD 8.6; median 22.5 years, range 11 days to $25.8 \mathrm{yrs}$ ) for those with possible epilepsy. At last follow-up, 201 (80.6\%) of those with definite epilepsy and 103 (86.6\%) of those with possible had been seizure free in the previous five years with 145 (72.4\% of those with definite epilepsy) and 90 (87.4\% of those with possible epilepsy) off AEDs.

Follow-up information is summarised in Table 16. 


\begin{tabular}{|c|c|c|}
\hline & Definite Epilepsy $(\mathrm{N}=564)$ & Possible Epilepsy $(\mathrm{N}=\mathbf{2 2 8})$ \\
\hline Male & $293(52.0 \%)$ & $95(41.7 \%)$ \\
\hline No seizures before Index & $263(46.6 \%)$ & $113(49.6 \%)$ \\
\hline \multicolumn{3}{|l|}{ Seizure (IS) } \\
\hline 1-5 seizures before IS & $233(41.3 \%)$ & $78(34.2 \%)$ \\
\hline 6-10 seizures before IS & $43(7.6 \%)$ & $26(11.4 \%)$ \\
\hline$>10$ seizures before IS & $24(4.4 \%) 1$ unknown & $11(4.8 \%)$ \\
\hline \multicolumn{3}{|c|}{ Number of seizures during total follow-up } \\
\hline No further seizures after IS & $205(36.3 \%)$ & $115(50.4 \%)$ \\
\hline 1-5 further seizures & $171(30.3 \%)$ & $54(23.7 \%)$ \\
\hline 6-10 further seizures & $39(6.9 \%)$ & $12(5.3 \%)$ \\
\hline 11-15 further seizures & $14(2.5 \%)$ & $8(3.5 \%)$ \\
\hline$>15$ further seizures & $132(23.4 \%) .3$ unknown & $39(17.1 \%)$ \\
\hline Follow-up at 5 years ${ }^{1}$ & $\mathrm{~N}=459$ & $\mathrm{~N}=193$ \\
\hline No seizures in past 5 years & $176(38.3 \%)$ & $104(53.9 \%)$ \\
\hline $1-5$ seizures & $145(31.6 \%)$ & $46(23.8 \%)$ \\
\hline 6-10 seizures & $34(7.4 \%)$ & $13(6.7 \%)$ \\
\hline $11-15$ seizures & $11(2.4 \%)$ & $5(2.6 \%)$ \\
\hline$>15$ seizures & $91(19.8 \%)$ & $25(13.0 \%)$ \\
\hline Follow-up at 10 years ${ }^{2}$ & $\mathrm{~N}=414$ & $N=175$ \\
\hline No seizures in past 5 years & $275(66.4 \%)$ & $128(73.1 \%)$ \\
\hline $1-5$ seizures & $67(16.2 \%)$ & $26(14.9 \%)$ \\
\hline 6-10 seizures & $18(4.3 \%)$ & $4(2.3 \%)$ \\
\hline $11-15$ seizures & $3(0.7 \%)$ & $1(0.6 \%)$ \\
\hline$>15$ seizures & $50(12.1 \%)$ & $15(8.6 \%)$ \\
\hline
\end{tabular}


Table 16: Seizure outcomes in those with definite and possible epilepsy

${ }^{1}$ Seizure outcome unknown in two people with definite epilepsy

${ }^{2}$ Seizure outcome unknown in one person with definite and one person with possible epilepsy

Estimation of seizure frequency in the first ten years was possible because GPs were sent yearly follow-up questionnaires up to 1997 allowing accurate estimation of seizure frequency during this time. The current follow-up represents one of only two further follow-ups undertaken after this point (the other was in 2001). Consequently the data were insufficient in some cases to accurate estimate seizure frequency beyond ten years and this was therefore not attempted.

In those classified as having definite epilepsy who had long term follow-up, 48 (19.1\%) had seizures in the last five years of whom 45 (93.7\%) were on AEDs. The index seizure was the first seizure in $17(35.6 \%)$. Of the 48, the aetiological classification (at 6 months) was idiopathic/cryptogenic in 37 (77.8\%); acute symptomatic seizures in three, remote symptomatic seizures in three and five had congenital deficits. Similarly for those classified as having possible epilepsy, $16(13.4 \%)$ had seizures in the last five years of whom all but one remained on AEDs. The index seizure was the first seizure in eight $(50 \%)$.

Seizure outcome in people for whom the index seizure was the first seizure is shown in Table 17. 


\begin{tabular}{llll}
\hline & $\begin{array}{l}\text { Complete Follow- } \\
\text { up (\%) }\end{array}$ & $\begin{array}{l}\text { Incomplete } \\
\text { Follow-up }\end{array}$ & Died \\
\hline Definite Epilepsy & $114(43.3 \%)$ & $35(13.3 \%)$ & $114(43.3 \%)$ \\
(N=263) & & & \\
Single Seizure & $43(37.7 \%)$ & $12(34.3 \%)$ & $65(57.0 \%)$ \\
Further Seizures & $71(62.3 \%)$ & $23(65.7 \%)$ & $49(43 / 0 \%)$ \\
Mean follow-up & $24.1(0.91)$ & $13.2(3.3)$ & $6.3(6.5)$ \\
(years) (SD) & & & \\
Possible Epilepsy & $60(53.1 \%)$ & $13(11.5 \%)$ & $40(35.4 \%)$ \\
(N=113) & & & \\
Single Seizure & $33(55 \%)$ & $9(69.2 \%)$ & $28(70 \%)$ \\
Further Seizures & $27(45 \%)$ & $4(30.8 \%)$ & $12(30 \%)$ \\
Mean follow-up & $24.1(0.97)$ & $13.2(2.4)$ & $6.0(5.8)$ \\
(years) (SD) & & & \\
\hline
\end{tabular}

Table 17: Seizure outcomes in people in whom the index seizure was the first seizure

*These results are discussed in conjunction with a previous published report (Hart et al., 1990) on page 211. 


\subsubsection{Survival analysis of time to first seizure and Cox regression model}

The probabilities of relapse after the index seizure for those with definite, possible and febrile seizures are shown in Figure 5.

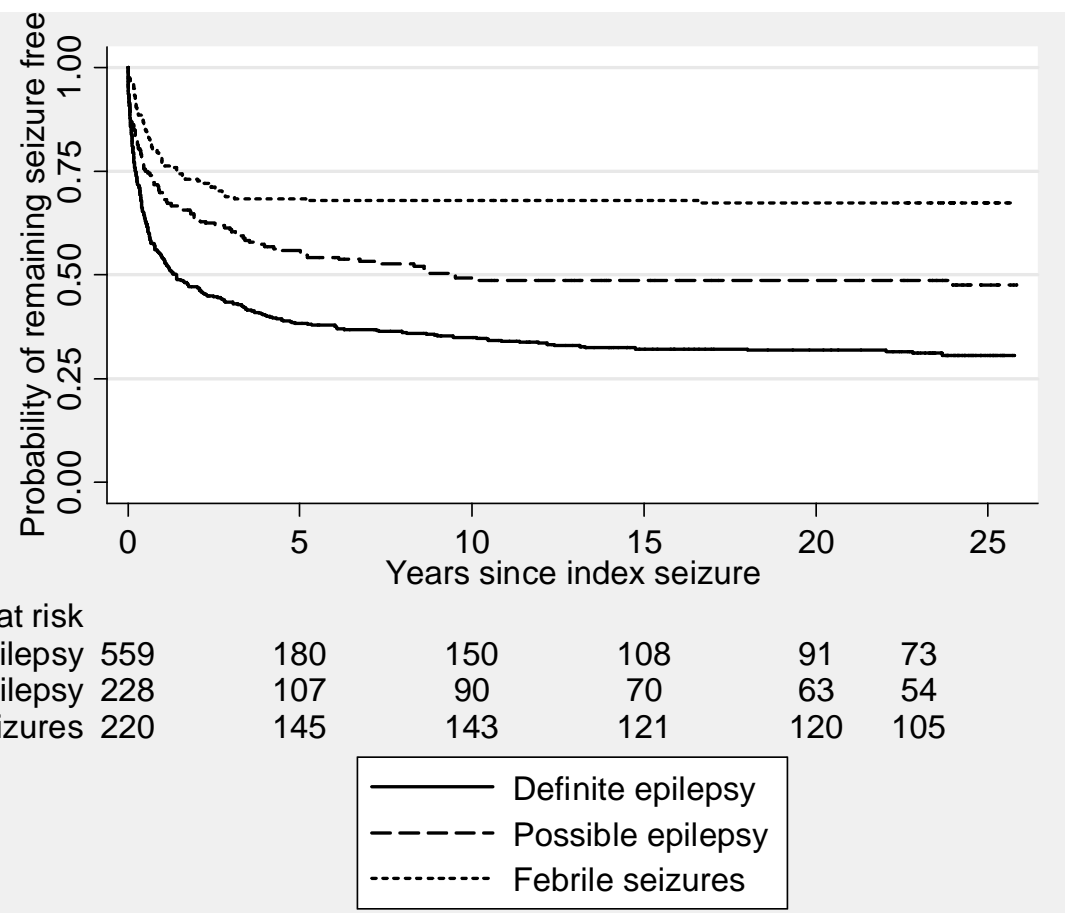

Figure 5: Kaplan-Meier analysis of probability of remaining seizure free after index seizure for those with definite, possible and febrile seizures

The risk of relapse is highest in the first five years with the probability of remaining seizure free appearing relatively stable 10 to 15 years after the index seizure for all seizure groups

The log-rank test was used to check the difference in the probability of relapse in those with definite and possible epilepsy $\left(\chi^{2}=19.3, \mathrm{df}=1, \mathrm{p}<0.001\right)$. People classified as having possible epilepsy were less likely to relapse than those with definite epilepsy (HR 0.63, 95\% CI 0.51, 0.78). 
In people with definite and possible epilepsy the only factor found to be associated with an increased risk of seizure recurrence was having seizures prior to the index seizure in the Cox regression model. For those in whom the index seizure was the first seizure, the risk of seizure recurrence was significantly lower than those who had seizures before the index seizure $(\mathrm{HR}=0.65,95 \%$ CI $0.54,0.78, \mathrm{p}<0.001)$. Neither age, gender nor seizure type significantly affected the probability of seizure recurrence.

The proportions of those with definite and possible epilepsy who attained five year seizure freedom in the first ten years with subsequent relapse rates are shown in Table 18. 


\begin{tabular}{|c|c|c|c|c|}
\hline & Complete & Incomplete & Died & Total (\%) \\
\hline & FU & FU & & \\
\hline Definite Epilepsy & $\mathrm{N}=\mathbf{2 5 2}$ & $\mathrm{N}=82$ & $\mathbf{N}=\mathbf{2 3 0}$ & $N=564$ \\
\hline SF 0-5 yrs then & $8(5$ in $\mathrm{TR}, 1$ & 5 & $4(3$ in $T R, 1$ & $14(8.1 \%)$ \\
\hline \multirow[t]{2}{*}{ relapse $5-10$ yrs } & $\mathrm{SF})$ & & $\mathrm{SF})$ & \\
\hline & $(\mathrm{N}=96)$ & $(\mathrm{N}=26)$ & $(\mathrm{N}=54)$ & $(\mathrm{N}=176)$ \\
\hline SF $0-10$ yrs then & 11 (5 in TR, & 0 & $2(1 \mathrm{SF})$ & $13(9.0 \%)$ \\
\hline \multirow[t]{2}{*}{ relapse thereafter } & $3 \mathrm{SF})$ & & & \\
\hline & $(\mathrm{N}=88)^{1}$ & $(\mathrm{~N}=21)$ & $(\mathrm{N}=35)^{3}$ & $(\mathrm{~N}=144)$ \\
\hline SF 5-10 yrs then & 11 (3 in TR, & 1 & $4(2 \mathrm{SF})$ & $16(9.5 \%)$ \\
\hline \multirow[t]{2}{*}{ relapse thereafter ${ }^{2}$} & $4 \mathrm{SF})$ & & & \\
\hline & $(\mathrm{N}=84)$ & $(\mathrm{N}=22)$ & $(\mathrm{N}=63)$ & $(\mathrm{N}=169)$ \\
\hline Possible Epilepsy & $N=119$ & $\mathbf{N}=\mathbf{3 3}$ & $N=76$ & $\mathrm{~N}=\mathbf{2 2 8}$ \\
\hline SF 0-5 yrs then & 9 (8 in TR $)$ & 2 (TR) & $2(1 \mathrm{SF})$ & $12(11.7 \%)$ \\
\hline relapse $5-10$ yrs & $(\mathrm{N}=68)$ & $(\mathrm{N}=18)$ & $(\mathrm{N}=18)$ & $(\mathrm{N}=104)$ \\
\hline \multirow[t]{2}{*}{ SF $0-10$ yrs } & $2(1 \mathrm{SF})$ & 0 & 0 & $2(2.2 \%)$ \\
\hline & $(\mathrm{N}=59)$ & $(\mathrm{N}=16)$ & $(\mathrm{N}=16)$ & $(\mathrm{N}=91)$ \\
\hline SF 5-10 yrs then & $2(1 \mathrm{TR})$ & 0 & $1(\mathrm{SF})^{4}$ & $2(7.1 \%)$ \\
\hline relapse thereafter & $(\mathrm{N}=28)$ & $(\mathrm{N}=8)$ & $(\mathrm{N}=5)$ & $(\mathrm{N}=41)$ \\
\hline
\end{tabular}

Table 18: Proportion of people with definite and possible epilepsy who attain at least five year seizure freedom in the first ten years of follow-up

Legend: $\mathrm{FU}=$ Follow-up, $\mathrm{TR}=$ Terminal Remission (five or more years seizure free up to the end of follow-up), $\mathrm{SF}=$ Seizure Freedom (one or more years seizure free but less than five years seizure freedom at last follow-up) 
${ }^{1}$ Calculated from the 96 people SF in the $1^{\text {st }}$ five years minus the eight people who had a recurrence in the next five years

${ }^{2}$ Refers to people not seizure free in the first five years but who were seizure free in the second five years of follow-up

${ }^{3} 15$ of those who were seizure free at $0-5$ years died before ten years of follow-up.

${ }^{4}$ Died within five years of last seizure

Only a small proportion of those who attained at least five years of continuous seizure freedom in the first ten years subsequently relapsed during follow-up (30/252 (11.9\%) of those with definite epilepsy; 13/119 (10.9\%) of those with possible epilepsy) of whom $13(43.3 \%)$ of those with definite and nine (69.2\%) with possible epilepsy had become seizure-free again and were in terminal remission (seizure-free for at least five years on or off AEDs) at last follow-up.

Seventy seven (30.6\%) of those with definite epilepsy and 57 (47.9\%) with possible epilepsy entered and remained in terminal remission after the index seizure while a further $73(29.0 \%)$ of those with definite and $26(21.8 \%)$ with possible epilepsy entered five year terminal remission within the first five years of the index seizure. Another 38 $(15.0 \%)$ of those with definite and $11(7.2 \%)$ with possible epilepsy entered terminal remission for the first time after more than ten years of follow-up. 
4.2.4.2 The impact of aetiology on prognosis in definite epilepsy

In those classified as having definite epilepsy, presumed aetiology affects the probability of seizure recurrence $(\mathrm{p}<0.001)$ (Table 19). Compared with those with idiopathic/cryptogenic epilepsy, people with remote symptomatic epilepsy had no difference in the probability of seizure relapse (HR 1.16, 95\% CI 0.89, 1.51). In contrast people with acute symptomatic epilepsy were less likely to relapse (HR 0.430 ; $95 \%$ CI $0.30,0.64)$ while those with epilepsy associated with a congenital deficit were more likely to relapse (HR 2.07, 95\% CI 1.21, 3.56) (Figure 6). 
134

\begin{tabular}{|c|c|c|c|c|}
\hline & $\begin{array}{l}\text { Idiopathic/Cryptogenic } \\
(\%)(\mathrm{N}=346)\end{array}$ & $\begin{array}{l}\text { Remote } \\
\text { Symptomatic } \\
(\%)(\mathrm{N}=119)\end{array}$ & $\begin{array}{l}\text { Acute } \\
\text { Symptomatic } \\
(\%)(\mathrm{N}=83)\end{array}$ & $\begin{array}{l}\text { Congenital } \\
\text { Deficit } \\
(\%)(\mathrm{N}=16)\end{array}$ \\
\hline Alive & $262(75.7 \%)$ & $21(17.6 \%)$ & $40(48.2 \%)$ & $11(68.8 \%)$ \\
\hline Complete & $193(73.7 \%)$ & $14(66.7 \%)$ & $34(85 \%)$ & $11(100 \%)$ \\
\hline \multicolumn{5}{|l|}{ Follow-up } \\
\hline Seizures in & $36(18.7 \%), 35(97.2 \%)$ & $3(21.4 \%)$, all & $3(8.8 \%)$, all & $5(45.5 \%), 4$ \\
\hline last 5 yrs & on $\mathrm{AEDs}^{1}$ & on $\mathrm{AEDs}^{2}$ & on AEDs & on AEDs \\
\hline $\mathrm{SF}<5 \mathrm{yrs}$ & $14(38.9 \%), 13$ on AEDs & 2 & 1 & 1 \\
\hline SF in last 5 & $47(24.4 \%)$ & $5(35.7 \%)$ & $4(11.7 \%)$ & 1 \\
\hline \multicolumn{5}{|l|}{ yrs, on } \\
\hline \multicolumn{5}{|l|}{ AEDs } \\
\hline SF in last 5 & $110(57.0 \%)$ & $6(42.9 \%)$ & $27(79.4 \%)$ & $5(45.5 \%)$ \\
\hline \multicolumn{5}{|l|}{ yrs, off } \\
\hline AEDs & & & & \\
\hline
\end{tabular}

Table 19: Seizure outcome in those classified as having definite epilepsy by aetiology

${ }^{1}$ Date of last seizure unknown in 12

${ }^{2}$ Date of last seizure unknown in one 


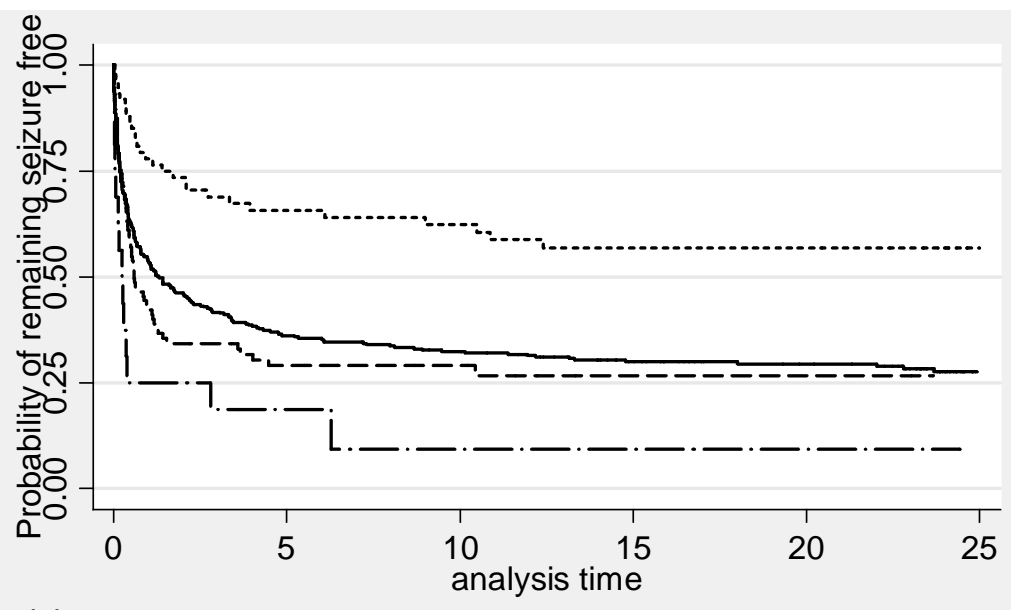

Number at risk Idiopathic/cryptogenic 344 Remote symptomatic 118 Acute symptomatic 81 Congenital 16

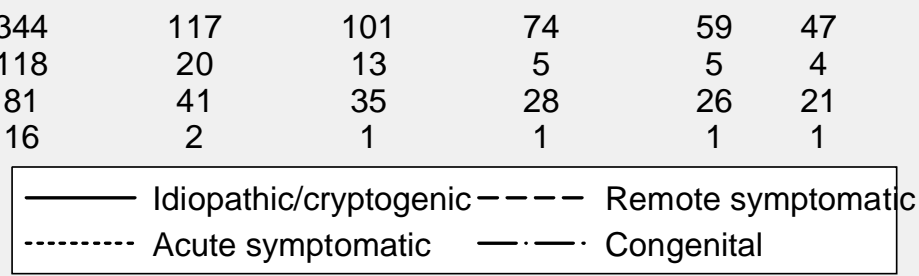

Figure 6: Kaplan-Meier analysis of probability of remaining seizure free after index seizure in those with definite epilepsy stratified by aetiological grouping 
In people with idiopathic/cryptogenic epilepsy, 47 were seizure free in the last five years but remained on AEDs. Of these eight had not had any further seizures since the index seizure (although all had seizures prior to the index seizure), $18(38.3 \%)$ had been seizure free for over 20 years and eight were seizure free for at least 15 years with all but two of the remainder seizure free more than ten years.

In those with remote symptomatic epilepsy, five were seizure free, on AEDs, in the last five years. Of these two had had no further seizures since the index seizure (both had seizures prior to the index seizure), while another two were seizure free more than 20 years. Four people with acute symptomatic seizures were seizure free on AEDs. Of these one only ever had one seizure (index seizure) while the remaining three were seizure free for more than twenty years at last follow-up.

One person with seizures as a result of a congenital deficit remained on AEDs despite being seizure free more than twenty four years. Of the 16 people with possible epilepsy with seizures in the last five years, at least three were seizure free for at least one year at the end of follow-up. 


\section{Chapter 5: The Study of Seizure Patterns in people with chronic epilepsy (Study 3)}

\subsection{Methods}

\subsubsection{Participants:}

Participants, who all gave informed consent, were recruited sequentially from two specialist epilepsy clinics at the National Hospital for Neurology and Neurosurgery between March 2008 and March 2010. Those eligible for inclusion had a history of established refractory epilepsy, defined as active epilepsy (seizures in the past two years) at least five years after onset and had been treated with at least two AEDs during that time. People in whom an alternative diagnosis or a diagnosis of non-epileptic seizures was considered, or who had had epilepsy surgery, were excluded. Data were collected in all cases by the completion of a short questionnaire by the participants, including a one-to-one interview to identify periods of remission (if any) as well as to ascertain seizure frequency in the previous year, and by a review of medical notes.

\subsubsection{Seizure patterns:}

Seizure patterns were determined. These were adapted from those previously described in a primary care study (Goodridge and Shorvon, 1983). Remission was defined as the presence of a period of at least two years during which no seizures occurred. All participants had active epilepsy at the time of inclusion in the study, and could be categorised into two groups according to seizure pattern: 
- continuous seizure pattern - no period of remission had occurred since the onset of the epilepsy

- an intermittent pattern - at least one period of remission had occurred since the onset of the epilepsy

In addition we identified a number of people who were in late terminal remission (two or more years seizure free at the time of enrolment) but who had had a previous continuous pattern.

Factors (age, age of onset, gender, seizure type, epilepsy syndrome, current and total number of AEDs, seizure frequency in the past year, aetiology, history of SE, Vagus Nerve Stimulator implantation) which were potential determinants of the different seizure patterns in people with refractory epilepsy, were investigated.

\subsubsection{Seizure and syndrome classification:}

The age of onset of seizures, the time since the last seizure (in days) and the predominant seizure type experienced by the individual were recorded. Seizures were classified according to the 1981 seizure classification (ILAE, 1981) (simple partial seizures, complex partial seizures or primary generalised and secondary generalised seizures) although a new classification for epileptic seizures and syndromes has recently been proposed (Berg et al., 2010). The epilepsies were classified as cryptogenic partial epilepsy, symptomatic partial epilepsy, idiopathic generalised epilepsy (IGE) or unclassified whether partial or generalised. Seizure frequency was classified into three categories: no seizures, 12 seizures or fewer, or more than 12 seizures in the previous year.

The information was entered into a secure anonymised database. 


\subsubsection{Ethical approval:}

The study was approved by the Joint Research Ethics Committee of the National Hospital for Neurology and Neurosurgery and the UCL Institute of Neurology. (REC reference $07 / \mathrm{H} 0716 / 99$ )

\subsubsection{Statistical analysis:}

Analysis was carried using STATA version 10 and SPSS v17. Categorical data was compared using the Chi-Squared test or, where appropriate, the Fisher's Exact Test. The number of current AEDs taken and total AEDs ever taken were grouped for analysis. Normally distributed data were analysed using Student's t-tests, while nonnormally distributed data were analysed using the Mann-Whitney test. All p-values were 2-sided. 


\subsection{Results}

We included 290 people, of whom 169 (58\%) were female in the study. The mean age was 41.0 (SD 12.3) years, with a mean age of onset of seizures of 14.9 (SD 10.3, median 13) years.

Seventy percent (204) of the participants had had a continuous pattern of seizures over the time of follow up (i.e. had had no periods of remission of 2-years or more) and 86 $(30 \%)$ had an intermittent pattern (i.e. with at least one previous period of remission). In these two groups, the demographics and clinical features (Table 20), the seizures types (Table 21) and seizure frequencies (Table 22) are shown. 


\section{Continuous Pattern}

$(\mathrm{N}=204)$

Mean 8.1, SD 4.1,

median 8 (range 2-21)

Mean 2.4, SD 1.0,

median 2 (range 0-5)

$120(58.8 \%)$

Mean 40.5, SD 12.4

Median 39.5 (range 16-75)

Age of first seizure (years)

Duration of epilepsy (years)

Normal Imaging

History of SE

Learning Disability

VNS

Hippocampal Sclerosis

Cortical dysplasia

CVD

Multifocal pattern on EEG
Mean 15.6, SD 11.0

Median 14 (range 0.5-68)

Mean 24.9, SD 13.4,

Median 23.5,

(range 5-65)

$\begin{array}{ll}127(62.6 \%) & 56(65.9 \%) \\ 45(22.1 \%) & 16(18.6 \%) \\ 10(4.9 \%) & 2(2.3 \%) \\ 13(6.4 \%) & 1(1.2 \%) \\ 25(12.3 \%) & 13(15.1 \%) \\ 13(6.4 \%) & 3(3.5 \%) \\ 26(12.7 \%) & 7(8.1 \%) \\ 30(14.7 \%) & 9(10.5 \%)\end{array}$

Mean 6.6, SD 3.3,

median 6 (range 2-16)

Mean 2.2, SD 0.8, median 2 (range 1-4)

$49(57.0 \%)$

Mean 42.2, SD 12.2

Median 39 (range 24-69)

Mean 13.3, SD 8.3

Median 13 (range 0.5-39)

Mean 28.9, SD 13.6

Median 28.5 (range 5-60)

Table 20: Basic demographics and epilepsy features

Legend: Vagus Nerve Stimulation (VNS), Status Epilepticus (SE), Cerebrovascular Disease (CVD). 
Continuous Pattern Intermittent Pattern

$(\mathrm{N}=\mathbf{2 0 4}) \quad(\mathrm{N}=\mathbf{8 6})$

\begin{tabular}{lll}
\hline Seizure type & & \\
\hline Simple partial seizures & $19(9.3 \%)$ & $3(3.5 \%)$ \\
Complex partial seizures & $112(54.9 \%)$ & $43(50 \%)$ \\
Secondary generalised seizures & $38(18.6 \%)$ & $19(22.1 \%)$ \\
Generalised seizures* & $35(17.2 \%)$ & $21(24.4 \%)$ \\
\hline Syndrome & & \\
\hline IGE & $32(15.7 \%)$ & $18(20.9 \%)$ \\
CPE & $92(45.1 \%)$ & $39(45.3 \%)$ \\
SPE & $74(36.3 \%)$ & $23(26.7 \%)$ \\
Unclassified & $6(2.9 \%)$ & $6(7.0 \%)$ \\
\hline
\end{tabular}

Table 21: Frequency of the predominant seizure type and epileptic syndromes in seizure patterns

*Primary generalised seizures or generalised not determined if focal or generalised in onset

Legend: Idiopathic Generalised Epilepsy (IGE) Cryptogenic Partial Epilepsy (CPE), Symptomatic Partial Epilepsy (SPE) 


\begin{tabular}{llll} 
Seizure Pattern & No seizures in last & $\leq \mathbf{1 2}$ seizures in & $>12$ seizures in \\
& year (\%) & last year $(\%)$ & last year (\%) \\
\hline Continuous & $5(2.5 \%)$ & $45(22.1 \%)$ & $154(75.5 \%)$ \\
Intermittent & $6(7 \%)$ & $37(43.0 \%)$ & $43(50.0 \%)$ \\
\hline
\end{tabular}

Table 22: Seizure frequency in the previous 12 months in people with continuous and intermittent seizures patterns

Neither current age $(p=0.26)$ nor age of onset $(p=0.22)$ was significantly different between those with continuous and intermittent seizure patterns. The total number of AEDs ever taken was significantly different between the two groups, being higher in the continuous group than in the intermittent (chi squared $=11.2, \mathrm{df}=3, \mathrm{p}=0.01$ ). (Duration of epilepsy differed between the two groups (see table 1), but as this was not an independent variable, it was not included in this analysis). People with a continuous pattern also had more seizures in the past year than those in the intermittent group (Chi squared $=18.5, \mathrm{df}=2, \mathrm{p}<0.001)($ Table 21$)$.

In those who had a continuous pattern of seizures, $81 \%$ (166) had never experienced even a six-month period of seizure freedom.

In the intermittent pattern group, the majority $(81.4 \%)$ had had only one period of remission. For 29 people (33.7\%) in the intermittent group, the first (or only) remission started within the first five years after seizure onset (early remission). Comparing those with early and late remissions (starting after 5 years duration), there was no difference in seizure severity, total or current number of AEDs, age or age of onset. 
A further 32 people who were in late terminal remission (who had a history of continuous seizures for at least five years and who then entered terminal remission (seizure free for two or more years at the time of the study)) were identified. These people did not meet the inclusion criteria and were excluded from the comparative analysis. The median number of AEDs tried was 6 (IQR 4.5, 8; range 2 to 11) and the median number of current AEDs was 2 (range 0 to 3). 


\section{Chapter 6: The Study of the Impact of Medication change in People with Chronic Epilepsy (Study 4)}

\subsection{Methods}

The methodology of the study of the study has been previously described (Luciano and Shorvon, 2007). People were retrospectively identified who had chronic uncontrolled epilepsy and who were commenced on a new AED (265 drug changes in 155 people) during a 24-month period (01/01/2002 - 31/12/2003). All had to fulfil the following criteria: (1) have active epilepsy (defined as at least one seizure a month in the previous three months) (2) a history of epilepsy for at least five years (3) age 16 years or older at the time of inclusion (4) a minimum follow-up in clinic of 12 months after the drug change. (5) a response to treatment that was easily quantifiable. They were excluded if there was a suspicion of non-epileptic seizures, a history of poor drug adherence or poor outpatient attendance.

The seizure frequency in the three months prior to starting on the first new AED was recorded and was used as the baseline seizure frequency for comparison throughout follow-up. Follow-up in this cohort was previously reported up to the end of June 2005. Follow-up is now extended follow up to the end of December 2009 or time of death. Those who subsequently underwent epilepsy surgery (excluding Vagus Nerve Stimulators (VNS) implantation) were followed up to the time of surgery.

The primary endpoint was the response to the addition of the new AED compared with the baseline seizure frequency recorded. Response was categorised into three groups: 1) Seizure free (for one year or more at last follow-up 2) 50-99\% reduction in seizure 
frequency compared with baseline seizure frequency and 3) less than $50 \%$ reduction in seizure frequency.

In the previous analysis, three factors (duration of epilepsy, number of previous AEDs tried and epilepsy syndrome) were found to be predictive of subsequent seizure freedom. This analysis was repeated using the duration of epilepsy, the syndrome and the number of previous AEDs at the time of the first drug change to predict seizure outcome at the end of follow-up. Other factors such as age, age of onset, seizure type, sex or number of concomitant AEDs were also analysed to determine if they influenced seizure prognosis. In contrast, the drug added was previously not found to influence seizure outcome.

Analysis was carried out using STATA version 10 and SPSS version 17. Categorical data was compared using the Chi-Squared test or, where appropriate, the Fisher's Exact Test. Seizure outcomes at the end of the initial and later follow-up periods were compared using the Stuart-Maxwell test. Kaplan-Meier analysis was used to estimate time to seizure recurrence in those who became seizure free (for one year or more). Time to event was defined as the duration from the date of first seizure freedom to the date of seizure relapse or to the date of the last clinic visit if seizure relapse had not occurred. 


\subsection{Results}

Of the original 155 registered in the study, further follow-up data was available on 139 (90\%), of whom 67 (48\%) had a greater than 50\% reduction in seizure frequency at follow-up in 2009.

Two people (1.4\%) died and five people (3.6\%) underwent epilepsy surgery during the follow-period. Complete follow-up to $31^{\text {st }}$ December 2009 was achieved in $118(85 \%)$. The mean follow up was 6.7 (SD 1.1, median 6.9) years. In total 448 drugs changes (Table 23) were made (468 in the original cohort of 155) of which $228(50.9 \%)$ were drug switches. In addition, 12 people had a VNS implanted. 


\begin{tabular}{|c|c|}
\hline Drug & Number of changes $(n=448)$ \\
\hline Levetiracetam & 106 \\
\hline Clobazam & 51 \\
\hline Pregabalin & 50 \\
\hline Topiramate & 41 \\
\hline Zonisamide & 38 \\
\hline Lamotrigine & 23 \\
\hline Sodium Valproate & 22 \\
\hline Carbamazepine & 19 \\
\hline Oxcarbazepine & 19 \\
\hline Phenytoin & 19 \\
\hline Lacosamide & 17 \\
\hline Tiagabine & 15 \\
\hline Gabapentin & 10 \\
\hline Acetazolamide & 7 \\
\hline Clonazepam & 3 \\
\hline Phenobarbital & 2 \\
\hline Primidone & 2 \\
\hline Lorazepam & 1 \\
\hline Midazolam & 1 \\
\hline Rufinamide & 1 \\
\hline Vigabatrin & 1 \\
\hline
\end{tabular}

Table 23: List of drug changes (AEDs started) in the 139 people with extended followup 
The drug with the highest proportion of positive responses was Carbamazepine (47\%) followed by Levetiracetam (45\%) but there were no statistically differences in response rates.

The treatment responses are summarised in Table 24.

\begin{tabular}{ll}
\hline Seizure Outcome & Total n $(\%)(\mathrm{N}=448)$ \\
\hline Less than 50\% Reduction in Seizure & $336(75 \%)$ \\
Frequency & \\
$50-99 \%$ Reduction in Seizure Frequency & $74(17 \%)$ \\
Seizure Freedom & $37(8 \%)(\text { AED changes in } 33 \text { people })^{2}$
\end{tabular}

Table 24: Seizure outcome following individual medication changes in the 139 people with prolonged follow-up.

${ }^{1}$ Seizure outcome unknown in one person following one additional medication change. ${ }^{2}$ Four people who had at least one year seizure freedom on one AED (one person having separate periods of seizure freedom), attained a further period of seizure freedom on addition of a further AED (the original AED being discontinued because of relapse or side-effects)

Seizure outcome at the end of the original follow-up was strongly predictive of outcome at the end of the later follow-up (Stuart-Maxwell $\mathrm{p}=0.03$ ). In particular, $64 \%$ of those who were seizure-free at the end of the original follow-up were seizure-free at last follow-up. Similarly $69 \%$ of those with less than $50 \%$ improvement remained so at last follow-up. Outcomes are summarised in Table 25. 


\begin{tabular}{lll}
\hline \multicolumn{1}{c}{ Outcome in 2004 } & \multicolumn{1}{c}{ Outcome in 2009 } & N \\
\hline Seizure free $(\mathrm{N}=25)$ & Seizure free & 16 \\
& $>50 \%$ improvement & 8 \\
& $<50 \%$ improvement & 1 \\
$>50 \%$ improvement $(\mathrm{N}=26)$ & Seizure free & 3 \\
& $>50 \%$ improvement & 13 \\
& $<50 \%$ improvement & 10 \\
$<50 \%$ improvement $(\mathrm{N}=88)$ & Seizure free & 7 \\
& $>50 \%$ improvement & 20 \\
& $<50 \%$ improvement & 61 \\
\hline
\end{tabular}

Table 25: Seizure outcomes in 2004 and 2009 in the cohort of 139 people

In contrast, the epilepsy syndrome (Chi squared $=5.69, \mathrm{df}=4, \mathrm{p}=0.2)$ and the duration of epilepsy ( $<$ or $\geq 10$ years) (chi squared $=0.05, \mathrm{df}=2, \mathrm{p}=0.97$ ) were no longer predictive of seizure outcome while the number of previous AEDs tried at study entry ( $<$ or $\geq 5$ AEDs) was of borderline statistical significance $($ chi squared $=5.9, \mathrm{df}=2$, $\mathrm{p}=0.052)$.

Seven of those seizure-free at last follow-up had five or more previous drug failures, and two of these had tried more than six previous AEDs (the number of previous AED trials was unknown in one).

As in the previous analysis age, age of onset, gender or seizure type, were not associated with seizure outcome. In contrast the number of concomitant AEDs $(0,1$ or 2 or more concomitant AEDs) was associated with seizure prognosis (Chi-squared $=10.5$, 
$\mathrm{df}=4, \mathrm{p}=0.033$ ), with those taking two or more AEDs less likely to attain seizure freedom.

VNS was implanted in 12 people with severe refractory epilepsy as evidenced by a mean duration of epilepsy of 30.7 (SD 13.0, median 30.5) years, a median of 13.5 AEDs tried (range 10 to 16) and two concomitant AEDs (range 1 to 4). VNS implantation resulted in a greater than $50 \%$ reduction in seizure frequency in one while another person reported some symptomatic improvement (seizures less severe and shorter in duration) without a significant reduction in seizure frequency.

Two people died during the study; one had a greater than $50 \%$ reduction in seizures up to the time of death. The other person continued to have severe refractory epilepsy without improvement up to the time of death, despite an initial improvement in seizure control. Twenty five people were seizure free at the end of the previous follow-up in 2004. Of these, $16(64 \%)$ were seizure free at the end of the current follow-up; 11 $(69 \%)$ of these remained seizure free throughout, with the remaining five people relapsing and subsequently re-entered remission. (One person had three separate periods of at least one year of seizure freedom).

In addition to the 35 people seizure free at the start or the end of the current follow-up, there were an additional 13 people (9.4\%) who had a significant period of seizure freedom of at least six months (range 6 to 10 months - three people) or one or more years (range 1 to 3.75 years - ten people) during the follow-up period but who were not seizure free at either end-point.. Consequently 45 (32\%) people in the cohort had at least one year of seizure freedom during follow-up; 33 (73.3\%) occurred in the context of a clear medication change. Two of this group (plus one other person without any 
significant period of seizure freedom) had complete cessation of their generalised seizures as a result of a medication change but had a recurrence of myoclonus. In total 20 had remained seizure free up to the end of follow-up after entering remission with a mean period of seizure freedom of 4.9 (SD 2.09) years.

Time to relapse in those who had a period of seizure-freedom is shown in Figure 7.

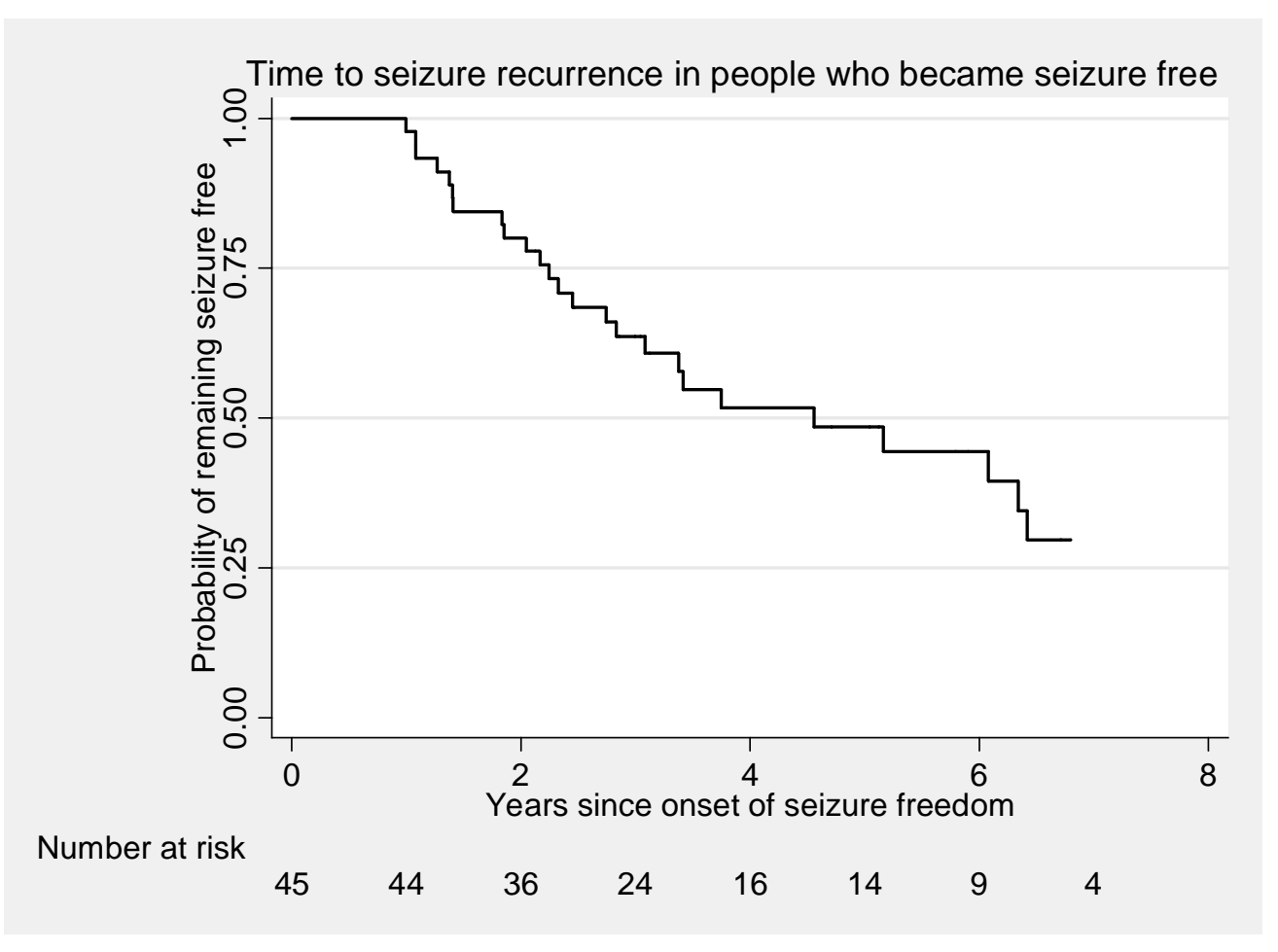

Figure 7: Kaplan-Meier analysis of the probability of remaining seizure free

Of the 45 people who achieved at least one year of seizure freedom during the course of follow-up, 25 (55.6\%) subsequently relapsed. Comparing those who remained seizure free $(n=20)$ with those who eventually relapsed $(n=25)$ neither epilepsy syndrome $(\mathrm{p}=0.33)$ nor the number of previous AEDs $(<$ or $\geq 5$ AEDs, $\mathrm{p}=0.37)$ were predictive of subsequent relapse. In contrast, those who had a duration of epilepsy of $\geq 10$ years and 
became seizure free were less likely to relapse (43.3\%) than those with an epilepsy duration of $<10$ years $(83.3 \%)(\mathrm{p}=0.02)($ Figure 8$)$.

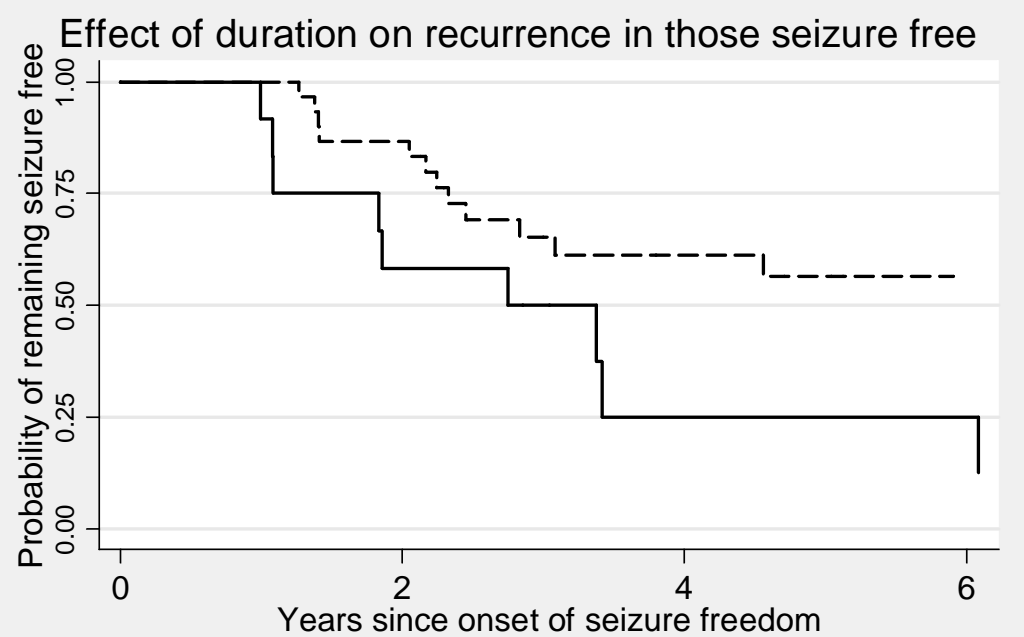

Number at risk

Duration <ten years 12

Duration $>=$ ten years 30

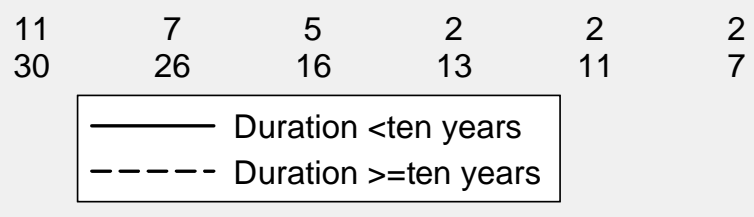

Figure 8: Kaplan-Meier analysis of the probability of remaining seizure free comparing the effect of epilepsy duration 


\section{Chapter 7: The Systematic Review of the Prognosis of Convulsive Status Epilepticus (Study 5)}

\subsection{Methods: search criteria}

To carry out this review, I searched Medline and PubMed for all papers published from January $1^{\text {st }} 1990$ up to December $31^{\text {st }} 2009$ with the terms "status epilepticus" combined with the terms "outcome", "mortality", "morbidity", "fatality", "prognosis", “coma”, "death" and “incidence", "prevalence" and "epidemiology". Only papers written in English and French were reviewed. From an initial 3,043 articles identified, all but 529 articles were excluded on the basis of the title and abstract.

A further search of Medline and PubMed was carried out for all papers published from January $1^{\text {st }} 1990$ up to December $31^{\text {st }} 2009$ with the terms "status epilepticus" combined with the terms “mortality”, “death", “duration" "EEG”, “outcome”, “morbidity”, "prognosis”, “coma", "cognition ".and "intellectual decline". Only papers written in English and French were reviewed. From an initial 3,311 articles identified, all but 437 articles were excluded on the basis of the title and abstract.

Exclusion criteria included case reports, review articles, commentaries, treatment protocols and studies with fewer than 30 people. Papers specifically concerned with non-convulsive SE were excluded but papers where figures for convulsive and non-convulsive SE were given were considered. Inevitably a distinction between convulsive and non-convulsive SE was not 
always made in some series and these papers are included. Both prospective and retrospective population- and hospital-based series were included. The search identified seven populationbased studies with the remainder of the studies cited being hospital-based. The reference lists of the identified articles were then examined for additional relevant studies. Common causes of SE were taken to be causes listed in at least one of the population-based studies of SE which had an incidence of greater than one percent.. The major studies of convulsive SE in the developing world are also reviewed in order to identify the predominant aetiologies of convulsive SE, although no attempt is made to analyse prognosis by aetiology, as is done for studies from the developed world.

Status epilepticus was defined according to the ILAE guidelines for epidemiologic studies on epilepsy as a single seizure of at least 30 minutes duration or a series of epileptic seizures during which function is not regained between ictal events in a period of at least 30 minutes (Commission on Epidemiology and Prognosis ILAE, 1993). This definition was adapted by the vast majority of studies reviewed, when a definition was given. 


\subsection{Major aetiologies in status epilepticus}

In examining the seven population-based studies of SE (Table 26, Page 157), 11 major causes (plus cryptogenic/idiopathic) were identified, with the relative frequencies for each cause shown in Table 25.

The comparative figures for each cause, taken from several studies looking at the incidence, aetiology and prognosis of SE from Africa, Trinidad, India and more recently China, are shown in table 26 . All of these studies were case series, not epidemiologically based, and although the range of aetiologies was very different from those in Western Europe and the US, it was not possible to derive any figures of frequency. Thus, although it seems likely that frequency is higher in the developing world primarily because of the high rate of infection, there are no figures to confirm this.

The prognosis of SE, in relation to each specific aetiology or, where appropriate, two aetiologies together, is reviewed here. 


\begin{tabular}{|c|c|c|c|c|c|c|c|}
\hline & Richmond $^{2}$ & Rochester & Switzerland $^{3}$ & Hessen & California & Bologna & London $^{8}$ \\
\hline Febrile & - & $8 \%$ & $14.9 \%{ }^{5}$ & $0 \%$ & $2.5 \%$ & - & $32 \%$ \\
\hline CVA & $10 \%(\mathrm{P}) / 22 \%(\mathrm{~A})$ & $19.1 \%$ & $30.5 \%{ }^{6}$ & $66.7 \%{ }^{1}$ & $12.4 \%$ & $41 \%$ & $0.5 \%$ \\
\hline Trauma & $3 \%(\mathrm{~A})$ & $4.5 \%$ & - & $7.3 \%$ & $0.4 \%$ & $10 \%$ & $1.5 \%$ \\
\hline CNS Tumours & $7 \%(\mathrm{~A})$ & - & - & $12 \%$ & $1.8 \%$ & $5 \%$ & - \\
\hline Metabolic disturbance & $5 \%(\mathrm{P}) / 15 \%(\mathrm{~A})$ & $3.5 \%$ & - & $8.7 \%$ & $8.7 \%$ & $24 \%{ }^{7}$ & $3 \%$ \\
\hline Medication induced/overdose & $2 \%(\mathrm{P}) / 3 \%(\mathrm{~A})$ & $2 \%$ & - & $10.7 \%$ & - & - & $1 \%$ \\
\hline \multirow[t]{2}{*}{ Anoxia/Hypoxia } & $5 \%(\mathrm{~A})$ (anoxia) & $10 \%$ & Excluded & - & $8 \%$ & $9.1 \%$ & $0.5 \%$ \\
\hline & $5 \%(\mathrm{P}) / 13 \%$ (A) (hypoxia) & & & & & & \\
\hline Cryptogenic & $5 \%(\mathrm{P}) / 3 \%(\mathrm{~A})$ & $13.5 \%$ & $8.7 \%$ & $8.7 \%$ & - & $11 \%$ & $7 \%$ \\
\hline
\end{tabular}


Table 26: Major Aetiologies of Status Epilepticus ${ }^{1}$

$\mathrm{A}=$ adults $; \quad \mathrm{P}=$ paediatric

${ }^{1}$ Some studies only give percentages for some aetiologies

${ }^{2}$ In the Richmond study, aetiologies were given separately for the paediatric and adult populations

${ }^{3}$ In the EPISTAR study, aetiologies were given separately for people with and without a history of epilepsy

${ }^{4}$ Percentage of the total cohort. (Low AEDs was the cause of SE in $18.9 \%$ of people with epilepsy)

${ }^{5}$ People with prior history of epilepsy only

${ }^{6}$ People with no prior history of epilepsy only

${ }^{7}$ Combination of systematic metabolic disorders and postanoxic encephalopathy

${ }^{8}$ Paediatric population 


\begin{tabular}{|c|c|c|c|c|c|c|c|c|c|c|c|c|}
\hline & Nigeria & Trinidad & Brazil & Tunisia & India & Senegal & Thailand & Iran & Congo & Kenya & Ethiopia & China \\
\hline Study design & $\mathrm{R}$ & $\mathrm{R}$ & $\bar{P}$ & $\mathrm{R}$ & $\bar{P}$ & $\bar{R}$ & $\mathrm{R}$ & $\mathrm{R}$ & $\mathrm{R}$ & $\mathrm{R}$ & $\overline{\mathrm{R}}$ & $\mathrm{R} / \mathrm{P}$ \\
\hline \multirow[t]{2}{*}{ Duration } & 10 & 18 & 1989- & 1990- & 1994- & 1988- & 1981- & 1999- & 1998- & $2002-$ & 1997- & 1996- \\
\hline & years & months & 1993 & 1997 & 1996 & 1998 & 2000 & 2004 & 2003 & 2003 & 2007 & 2007 \\
\hline Number & 41 & 41 & 111 & 139 & 85 & 697 & 32 & 135 & 607 & $388^{3}$ & 119 & 220 \\
\hline History of & - & - & $59.4 \%$ & $10 \%$ & $20 \%$ & $9.9 \%$ & $75 \%$ & $37 \%$ & $14.5 \%$ & $30.8 \%$ & $38.7 \%$ & $50 \%$ \\
\hline seizures & & & & & & & & & & & & \\
\hline \multicolumn{13}{|l|}{ Aetiology } \\
\hline Febrile seizures & - & $5 \%$ & $5.4 \%$ & $41 \%$ & - & $8.6 \%$ & $0 \%$ & $51.1 \%$ & & & - & \\
\hline AED non & & - & $18.9 \%$ & $10 \%$ & $20 \%$ & $9.9 \%$ & $9.4 \%$ & $28.2 \%$ & $3.8 \%$ & $5.9 \%$ & $17.6 \%$ & $15.5 \%$ \\
\hline compliance & & & $(31.8 \%)$ & & & & & & & & & \\
\hline CNS Infections & $41.5 \%$ & $15 \%^{2}$ & $10.8 \%$ & $18.7 \%$ & $28 \%$ & $67 \%$ & $65.6 \%$ & $7.4 \%$ & $74.5 \%^{1}$ & $8.0 \%^{4}$ & $36.1 \%$ & $32.7 \%$ \\
\hline Metabolic & $34.1 \%$ & & $10.8 \%$ & $10.1 \%$ & $11 \%$ & $4.3 \%$ & $0 \%$ & $2.9 \%$ & - & & $13.4 \%$ & $11.4 \%^{6}$ \\
\hline Stroke & $14.6 \%$ & & $9.9 \%$ & $5.8 \%$ & $15 \%$ & $8 \%$ & $0 \%$ & $0 \%$ & - & & $12.6 \%$ & $10.90 \%$ \\
\hline Progressive & $9.8 \%$ & - & $2 . \%$ & $7 \%$ & - & $1.2 \%$ & $3.1 \%$ & $0 \%$ & - & $=$ & $10.9 \%$ & - \\
\hline \multicolumn{13}{|l|}{ Symptomatic } \\
\hline Remote & - & $51 \%$ & - & $6 \%$ & $7 \%$ & - & $0 \%$ & $0 \%$ & - & - & - & - \\
\hline \multicolumn{13}{|l|}{ Symptomatic } \\
\hline Idiopathic & - & $29 \%$ & $25.3 \%$ & $6 \%$ & $19 \%$ & - & $18.8 \%$ & $8.9 \%$ & - & & $16.8 \%$ & $7.7 \% \%^{5}$ \\
\hline
\end{tabular}




\begin{tabular}{|c|c|c|c|c|c|c|c|c|c|c|c|c|}
\hline Mortality & $100 \%$ & $0 \%$ & $19.8 \%$ & $15.8 \%$ & $10.50 \%$ & $24.8 \%$ & $6.3 \%$ & $12.6 \%$ & $26.5 \%$ & $15.2 \%$ & $20.2 \%$ & $15.9 \%$ \\
\hline Morbidity & - & $4.9 \%$ & - & $36 \%$ & & $13.6 \%$ & $\begin{array}{c}56.3 \% \\
(66.7 \% \\
\text { severe } \\
\text { n.d. })\end{array}$ & $27.3 \%$ & $2.9 \%$ & $11.9 \%$ & $3.4 \%$ & N/A \\
\hline $\begin{array}{l}\text { Inclusion } \\
\text { Criteria }\end{array}$ & $\begin{array}{c}\text { All } \\
\text { ages } \\
\text { (post- } \\
\text { Mortem } \\
\text { Study) }\end{array}$ & $\begin{array}{c}\text { All ages } \\
(51 \% \\
\text { less than } \\
10)\end{array}$ & $\begin{array}{l}\text { All } \\
\text { ages }\end{array}$ & $\begin{array}{l}\text { Study } \\
\text { in } \\
\text { infants } \\
\text { (mean } \\
\text { age } 11 \\
\text { months) }\end{array}$ & $\begin{array}{c}\text { All } \\
\text { ages } \\
\text { (mean } \\
\text { age 33) }\end{array}$ & $\begin{array}{l}\text { All } \\
\text { ages } \\
(61 \% \\
\text { aged } \\
\text { less } \\
\text { than } \\
10)\end{array}$ & $\begin{array}{c}\text { Paed } \\
\text { study } \\
\text { (mean } \\
\text { age } \\
6.5 \mathrm{yrs} \text { ) }\end{array}$ & $\begin{array}{l}\text { Paed. } \\
\text { Study }\end{array}$ & $\begin{array}{l}\text { Paed. } \\
\text { study }\end{array}$ & $\begin{array}{l}\text { Paed. } \\
\text { Study }\end{array}$ & $\begin{array}{l}\text { Children } \\
\text { aged } \\
<13 \\
\text { years } \\
\text { excluded }\end{array}$ & $\begin{array}{c}\text { All } \\
\text { ages } \\
(12 \% \\
\text { aged } \\
<16)\end{array}$ \\
\hline
\end{tabular}

$\mathrm{R}=$ Retrospective, $\mathrm{P}=$ Prospective. Paed.=Paediatric, $\mathrm{n} . \mathrm{d}=$ neurological deficit

${ }^{1}=$ In this study, 568 cases $(93.6 \%)$ were associated with fever, the common identified cause was malaria (68.5\%) (Cerebral or otherwise). Sepsis was responsible for $9.5 \%$ of cases.

${ }^{2}=15 \%$ of causes listed as acute symptomatic

${ }^{3}=$ Confirmed (155) or probable cases (233) of $\mathrm{CSE}^{4}=$ The primary causes of CSE in the study were infectious: malaria without febrile convulsions (FC) $29.4 \%$, malaria with FC $23.7 \%$, FC secondary to other infection $3.6 \%$ and acute bacterial meningitis $8 \%$

$5=$ The number of CNS tumours and developmental malformations combined

${ }^{6}=$ The number of toxic, hypoxia and metabolic cases combined

Table 27: Studies of Status Epilepticus in the developing world 


\subsection{Frequency and prognosis of different aetiologies}

\subsubsection{Stroke and status epilepticus}

In epidemiological studies stroke has been shown to be a significant cause of SE in the elderly particularly in those with no prior history of seizures (table 26). In the California study (Wu et al., 2002), acute stroke accounted for only $1.6 \%$ of cases but was associated with the second highest mortality rate (25.6\%). In a review of 193 people with refractory SE, stroke was the underlying cause in $20 \%$ (Claassen et al., 2002).

In a population-based study from Germany (Knake et al., 2006), the long-term mortality rate in people with a first episode of cerebrovascular-related SE was 57\% compared with $48 \%$ in people with acute stroke without SE. Multivariate analysis showed that, after six months, people with SE had twice the risk of death compared with people with stroke but without SE (hazard ratio of $2.12,95 \%$ CI 1.04, 4.32, $\mathrm{p}=0.039$ ).

In a comprehensive study of the frequency of SE and stroke, 3,205 people with first time strokes over an 8-year period were reviewed (Rumbach et al., 2000). Of these, 159 had first time post-stroke seizures and SE was diagnosed in 31 people, with SE being the presenting symptom of epilepsy in 17 (SE occurring within 14 days of the stroke in seven). In four people stroke began with SE, and in the remaining ten people SE developed after one or more seizures. After a follow-up of 47 months, 15 people had died, with five deaths directly related to SE. Additional seizures occurred in eight of the initial SE cases and in all 14 people in whom SE was not the first post-stroke manifestation of epilepsy.. The study concluded that SE in stroke has a poor prognosis 
but that initial SE as a first epileptic symptom of epilepsy was not predictive of developing subsequent seizures (Rumbach et al., 2000).

A smaller study looked at stroke as a remote symptomatic cause of SE in a cohort of people with post-stroke first time seizures. Of 1,174 people with stroke, 180 had seizures. Of these 180, 17 (9\%) developed SE. The risk of developing SE was higher in those with greater disability (Rankin scale $>3$, odds ratio 4.36). Recurrent SE occurred in five people, all of whom had a first episode of SE within seven days post-stroke. Early onset SE was associated with a higher risk of recurrence of SE $(p=0.003)$ and higher mortality ( $\mathrm{p}=0.04)$ (Velioglu et al., 2001).

Examination of the US Nationwide Inpatient Sample over an eight year period identified 718,531 hospitalisations of people with acute ischaemic stroke (AIS) of whom generalised convulsive SE (GCSE) developed in 1,415 (0.2\%) (Bateman et al., 2007). Of 102,763 who were admitted with intra-cranial haemorrhage (ICH), GCSE developed in $266(0.3 \%)$. In-hospital mortality was significantly higher in those with GCSE as well as AIS or ICH, particularly if analysis was restricted to people with length of stay greater than one day. (AIS: $28.4 \%$ vs $9.2 \%$, p $<0.01$; ICH: $30.1 \%$ vs $24.3 \%, \mathrm{p}=0.03$ ). Other markers of morbidity such as pneumonia, the need for mechanical ventilation, tracheotomy and length of stay greater than seven days were all statistically associated with concomitant SE. In this study, no attempt at defining SE was made. In the Northern Manhattan Stroke Study (NOMASS), of 904 people with stroke, 37 (4.1\%) patients developed early seizures. SE occurred in ten people, $1.1 \%$ of the entire cohort of stroke patients and $27 \%$ of all patients with post stroke epileptic seizures (Labovitz et al., 2001). 
A cohort of 83 people with SE and stroke (44 with acute and 39 with remote stroke) and 159 people with acute stroke only (controls) were prospectively followed (Waterhouse et al., 1998). People with an acute stroke and SE had a mortality of 39\%, almost threetimes as high as in those with acute stroke only (14\%) or those with SE and remote stroke $(5 \%),(p<0.001)$. In addition there was almost an 8 -fold difference in mortality between the acute stroke and SE group and the remote stroke and SE group. This difference was not accounted for by age, sex or radiographic lesion size. Logistic regression analysis demonstrated a statistically significant synergistic effect of combined injuries of cerebral vascular ischaemia and SE (Waterhouse et al., 1998).

SE occurring after stroke is associated with a higher functional disability, and poor functional disability was the only identified predictor of SE in one study (Velioglu et al., 2001). This study found that early-onset SE (within the first seven days after stroke) was associated with a higher risk of further episodes of SE and higher mortality rate than those with late onset SE (Velioglu et al., 2001). Stroke severity and mortality were also found to be higher in people with early-onset SE in other studies (Rumbach et al., 2000; Afsar et al., 2003).

In a series from Turkey (Afsar et al., 2003), 121 people admitted to a single centre with SE over a three-year period were identified. The aetiology of the SE was stroke in 30 (24.8\%). Most people (22 (73\%)) had non-convulsive SE. Two-thirds were defined as early-onset (occurring with two weeks) and only ischaemic stroke was associated with late-onset SE. Overall mortality was $43.3 \%$, and disability (as defined by NIHSS scores) in the early-onset group was significantly associated with increased mortality $(p=0.02)$. This mortality figure is similar to that found for stroke-related SE $(26.3 \%)$ by others (Towne et al., 1994). In a retrospective study of 107 episodes of SE in China, 
cerebrovascular disease was the most common aetiology (27\%) and was the strongest predictor of poor outcome following SE with an odds ratio of 5.57 (95\% CI 1.45, 21.41, $p=0.001)$ (Hui et al., 2003).

In a large study on mortality rates in SE, based on hospital coding and without clear definitions made, cerebrovascular disease was a predictor of in-hospital mortality with an odds ratio of $2.08(95 \%$ CI $1.13,3.82)$ and a mortality rate of $22 \%(p<0.0001)$ and was also a predictor for the need for mechanical ventilation $(\mathrm{p}<0.0001$; Koubeissi and Alshekhlee, 2007).

\subsubsection{Alcohol, substance abuse and drug-induced status epilepticus}

Alcohol abuse (intoxication or withdrawal) has been found to be a common cause of SE in many population-based and hospital-based studies, with a reported range of 8.1 to $25 \%$ of SE caused alcohol, although alcohol was not reported to be a major cause in some studies (such as the Minnesota (Hesdorffer et al., 1998a) and EPISTAR (Coeytaux et al., 2000) studies. Alcohol-related SE is generally associated with a favourable outcome with most studies reporting a mortality rate of 0 to $10 \%(9.6 \%$ in the California study (Wu et al., 2002).

In an examination of the records of 249 adults with GCSE admitted to a single centre over a 12 year period, 27 people (10.8\%) in whom alcohol abuse was the only identifiable precipitating cause were identified (Alldredge and Lowenstein, 1993). In 12 (44\%), SE was the first presentation of alcohol-related seizures. At the time of discharge, $22(81.5 \%)$ had returned to baseline although time to gross recovery of mental status was more than 12 hours in 24 of the 27 . Four people $(14.8 \%)$ had new neurological deficits at the time of discharge; significant gait instability (one), moderate 
short-term memory deficits (one), and more diffuse cognitive dysfunction (two). The only death occurred in a 60 year old in whom the SE continued despite four hours of treatment (Alldredge and Lowenstein, 1993).

Drug toxicity or abuse is generally a more common cause of SE in hospital-based studies than in population-based studies, and the rate also varies markedly in different locations, with reported rates of 2 to $14 \%$ of cases. Cocaine (43\%) and theophylline (21\%) were the most commonly implicated drugs in the San Francisco study. Ten (71\%) of those with drug toxicity as a cause for SE had a favourable outcome. The mean duration of SE however, was markedly different in the four (29\%) with a poor outcome (severe neurological deficit or death; 1.9 hours) compared with people with favourable outcome (32.5 hours; Lowenstein and Alldredge, 1993). In a study of medically ill patients in ICU who developed SE approximately one month after admission, theophylline toxicity was found to be a primary or contributing cause in $25 \%$ and was associated with an overall mortality rate of $61 \%$ (Delanty et al., 2001). In another study drug toxicity-related cases of SE had a case fatality of $16.8 \%$ (Towne et al., 1994).

\subsubsection{Status epilepticus and antiepileptic drug reduction or withdrawal or low antiepileptic drug levels}

In people with a prior diagnosis of epilepsy, non-compliance with AEDs is often cited as the most common cause of SE (DeLorenzo et al., 1996). This may be the case in adults, but is not so in children. In the NLSTEPSS (Chin et al., 2006), only one case of convulsive SE was attributable to low antiepileptic drug concentrations, although low serum levels of AEDs were the cause in $21 \%$ of SE in children in the Richmond study (DeLorenzo et al., 1996). 
In older hospital-based series, 'non-therapeutic' anticonvulsant blood levels at the time of presentation was reported as the cause of SE in 3.9 to $34 \%$ of episodes of SE but with a low associated mortality (Shorvon, 1994).

The San Francisco Hospital Study found that 25\% of episodes of SE were related to withdrawal of AEDs, with $90 \%$ of people having a good outcome (defined as unchanged from baseline, or mild neurologic deficits that allowed independent living) such that the authors conclude "... that patients with a history of epilepsy who develop SE because of anti-convulsant drug withdrawal or break-through seizures can be expected to respond well to acute anticonvulsants" (Lowenstein and Alldredge, 1993). This is a conclusion which has been widely confirmed.

Several studies have looked at the different aetiologies of SE and the subsequent risk of developing SE refractory to standard first line treatments. In a study of 83 episodes of SE, low levels of AEDs were the primary cause of the SE in $27.7 \%$ of the nonrefractory cases but no refractory cases ( $\mathrm{p}<0.001$ ), allowing the authors to conclude that "SE caused by insufficient levels of AEDs is usually not refractory" (Holtkamp et al., 2005). Similarly low AED levels or a recent change in medication was found in $31 \%$ of people presenting in SE in $78 \%$ of whom the SE was non-refractory (Mayer et al., 2002). In a study looking at the use of propofol in 31 episodes of treatment resistant SE in 27 people, five people had SE secondary to AED withdrawal, all of whom had a good outcome (Rossetti et al., 2004). Low levels are often taken as a surrogate measure of poor compliance or drug reduction, but this is not always the case. 


\subsubsection{Severe acute cerebral anoxia/hypoxia and status epilepticus}

Anoxia, usually after cardiac arrest in adults, can result in deep coma with myoclonic jerking, and this is assumed by some authorities, but not all, to be a form of 'SE' (Shorvon, 1994). This dichotomy of opinion is evident by the fact that post-anoxic SE cases were excluded from the EPISTAR study (Coeytaux et al., 2000). In the population-based studies, anoxia is the cause in 8 to $13 \%$ of people with SE, with an associated mortality typically in the range of $60-80 \%$ although a mortality of $100 \%$ was reported in the Bologna study (four people) (Vignatelli et al., 2003). In this study the 30 -day case fatality rates were calculated with (39\%) and without (33\%) the post-anoxic patients.

In a study of 166 post-anoxic survivors of cardiac arrest treated with hypothermia, postanoxic SE was present in $24 \%$ with a mortality rate of $80 \%$ compared with the overall mortality rate of $71 \%(\mathrm{p}<0.001)$. Post-anoxic SE was associated with a higher mortality regardless of the type of acute cardiac rhythm or hypothermia treatment (Rossetti et al., 2007).

In a large study of mortality in convulsive SE hypoxia-ischaemic brain injury-associated SE was the strongest predictor of mortality with an odds ratio of 9.85 (95\% CI 6.63, 14.6) and a mortality rate of $69 \%$ and was a significant risk factor for the need for mechanical ventilation $(\mathrm{p}<0.0001)$ (Koubeissi and Alshekhlee, 2007). In an earlier study anoxia induced SE was responsible for $11.9 \%$ of all deaths with a mortality rate of $60 \%$ and was associated with the highest odds ratio for mortality (OR 8.2, $\mathrm{p}<0.0001)$ (Towne et al., 1994). 
In a study examining the outcomes of 114 people following cardiopulmonary resuscitation 50 (44\%) had seizures or myoclonus. SE occurred in $36(32 \%)$, of whom $10(9 \%)$ had SE other than Myoclonic (MSE), only one of whom was alive at six months. Nineteen people (17\%) had MSE, three (16\%) of whom were alive at discharge and none alive at six months. Seven $(6 \%)$ had status myoclonus other than MSE, none of whom were alive at discharge. When the subgroups with SE were considered individually, only MSE was associated with a significantly poorer survival $(\mathrm{p}<0.01)$ (Krumholz et al., 1988).

It is clear that people with MSE following cardiopulmonary resuscitation have a poorer prognosis than those without myoclonus. In a series of 50 people with myoclonus following resuscitation $45(90 \%)$ died within two weeks while the remainder remained in a permanent vegetative state (Thomke et al., 2005). Similarly in a series of 107 consecutive comatose patients after cardiac arrest, 40 had MSE. All patients with MSE died whereas 20 of the 67 people without MSE regained consciousness (Wijdicks and Young, 1994).

In the AAN practice parameter of the prediction of outcome of comatose patients following cardiopulmonary resuscitation, MSE within the first 24 hours following primary circulatory arrest was recognised as a predictor of poor outcome (Wijdicks et al. 2006).

\subsubsection{CNS infections, encephalitis and status epilepticus}

Acute CNS infections and encephalitis are important causes of SE particularly in children, typically accounting for about $1-12 \%$ of all episodes of SE in various series from the developed world, and are a more common cause of SE in children than in adults. In the California study, acute CNS infection was the cause of SE in $0.6 \%$ of 
cases with a median age of 42 years and a mortality rate of $32.6 \%$ (Wu et al., 2002). In the NLSTEPSS (Chin et al., 2006) 11 children (6\%) with SE had acute bacterial meningitis and seven (4\%) a viral CNS infection. Moreover three of the seven children who died had acute bacterial meningitis. The mortality rate for CNS infections in a large in-hospital study of SE was three percent (Koubeissi et al., 2007).

In a retrospective study of all admissions to a paediatric ICU in Montréal over a tenyear period, there were 147 admissions with SE of which $20(13.6 \%)$ were due to bacterial meningitis and $20(13.6 \%)$ due to encephalitis; both were associated with high morbidity and mortality (Lacroix et al., 1994). CNS infections were the cause of SE in $10.6 \%$ of cases in another study and were associated with a $71.4 \%$ mortality rate $(\mathrm{OR}=13.88, \mathrm{p}=0.014)$ (Sagduyu et al., 1998). CNS infections causing SE were found to be predictive of a poor outcome in a study from Hong Kong (adjusted OR 30.27, $\mathrm{p}=0.003$ ) (Hui et al., 2003).

The refractory nature of the SE induced by encephalitis and its rather poor outcome has been demonstrated in several hospital-based studies. In a review of 22 episodes of treatment resistant SE in children, presumed or agent-identified encephalitis was the underlying aetiology in ten children, four of whom died, five developed seizures and one returned to baseline (Sahin et al., 2001). Encephalitis was the cause of $22 \%$ of episodes of treatment resistant SE compared with only $4.3 \%$ of non treatment resistant SE $(p<0.18)$ (Holtkamp et al., 2005). In a case series of 17 episodes of treatment resistant SE in children, treated with a continuous midazolam, four had viral encephalitis, one of whom died, two developed moderate to severe encephalopathy and one made a full recovery (Yaffe and Lowenstein, 1993). 
In a review of all episodes of SE presumed to be caused by encephalitis in the paediatric intensive care unit over a four year period, 46 children with SE and encephalitis were identified. Of these $20(43.4 \%)$ developed treatment resistant SE with a 30\% mortality rate. Of the survivors, all but one developed subsequent epilepsy and/or neurological deficits, and none returned to baseline. For those with treatment responsive SE, four died, 16 developed epilepsy or had a neurological deficit and only six returned to baseline (Lin et al., 2008).

Population-based studies tend to show a rather more favourable outcome, with a lower frequency of treatment resistant SE. For instance, in the California Encephalitis Project (CEP) which is an ongoing project aimed at determining the cause of encephalitis, people identified with encephalitis were subdivided into three categories: refractory SE (defined as SE requiring anaesthetic coma for management (Group I); Non-refractory SE (Group II); and people without seizures (Group III). Four percent had refractory SE, $40 \%$ non-refractory SE and 56\% no seizures. People with refractory SE associated with encephalitis tended to be younger (median age 10 years) and had a poorer outcome with $28 \%$ dying within two years and $56 \%$ neurologically impaired or undergoing rehabilitation (Glaser et al., 2008).

In a study of the clinical characteristics and factors of post-encephalic epilepsy in 44 children from Taiwan, SE occurring as the first seizure $(\mathrm{p}<0.05)$ and herpes simplex encephalitis $(\mathrm{p}<0.01)$ were associated with a poor prognosis and an increased risk of developing intractable epilepsy (Chen et al., 2007). It should also be noted that there are two reports in the literature of people presenting with acute encephalitis associated with refractory, repetitive partial seizures (Saito et al., 2007). 


\subsubsection{Cerebral Tumour and status epilepticus}

Brain tumours are an uncommon cause of SE, representing two to five percent of episodes of SE in most studies although they were responsible for $12 \%$ of episodes of SE in the Hessen study (Knake et al., 2001). The associated mortality rates are 0 to $36 \%$.

One study has specifically looked at the clinical implications of SE in people with neoplasms (Cavaliere et al., 2006). Thirty five people were retrospectively identified with cerebral neoplasms and SE. Of these, $25(71.4 \%)$ had a primary brain tumour, the most common of which was a high-grade glioma (11) followed by low-grade glioma (6). Ten people had systemic tumours with presumed or identified cerebral metastases. Fifteen (43\%) had a prior history of seizures and 20 (57\%) were on AEDs at the time of SE. SE occurred at the time of diagnosis in ten (29\%), during tumour progression (23\%) or while the tumour was stable (23\%). Only one episode of SE occurred in a person during a confirmed period of tumour regression. At thirty days eight (23\%) people had died with four dying during the hospitalisation for SE. The only statistically significant factor in predicting 30-day mortality was tumour histology (three people [27\%] with primary brain tumours died compared with five people (50\%) with systemic tumours $(p=0.01)$. All episodes of SE were controlled. In further follow-up, age was associated with higher mortality (five people (71\%) older than 70 years and 15 people $(54 \%)$ less than 70 years died at a median of 44 and 154 days respectively, $p=0.049$ ) (Cavaliere et al., 2006).

\subsubsection{Trauma and status epilepticus}

Trauma is also an uncommon cause of SE, typically accounting for between 0 and $10 \%$ of episodes of SE in the major studies with an associated mortality of up to $20 \%$. SE as 
a result of head trauma was associated with $0 \%$ mortality in one large in-hospital study (Koiubeissi et al., 2007). In a series of 94 people with moderate to severe traumatic brain injury who underwent continuous EEG, seizures were detected in $21(22 \%)$, manifesting in six as focal motor twitching or atypical myoclonus and as nonconvulsive SE in two. All people with SE died, two as a result of sepsis following control of SE, three died as a result of progressive neurological deterioration and the final person died as a result of respiratory arrest following discharge (Vespa et al., 1999).

In a study to compare the risk of mortality and subsequent unprovoked seizures, 262 people with acute symptomatic seizures and 148 people with a first unprovoked seizure (all due to a static brain injury) were identified in Rochester. Traumatic brain injury (TBI) was the cause of provoked seizure in 91 (34.7\%) with 91 (34.7\%) being due to acute stroke and $80(30.5 \%)$ due to acute CNS infection. Of the 148 people with a first unprovoked seizure, 37 (25\%) had a previous history of TBI, $101(69.2 \%)$ had a previous stroke and $10(6.8 \%)$ had a previous CNS infection. Ten (11\%) people with acute TBI had SE which was significantly less than that seen with acute stroke (36.3\%) and acute CNS infections (58.8\%). Similarly those with a history of TBI with a first unprovoked seizure were less likely to present with SE (16.2\%) than those with a history of stroke (22.8\%) or CNS infection (90\%). The 30 day mortality was $11 \%$ in those with acute symptomatic seizure due to TBI while the ten year mortality was similar in those with acute symptomatic and unprovoked seizures with a history of TBI (Hesdorffer et al., 2009).

\subsubsection{Metabolic disorders and status epilepticus}

Metabolic disorders are the cause of SE in two to $15 \%$ of episodes of SE in reported series with an associated mortality rate of up to $31 \%$ (Towne et al., 1994). Acute 
metabolic disturbance (electrolyte imbalance, hypoglycaemia, hypocalcaemia or hypomagnesaemia) was the aetiology of SE in approximately three percent of children in NLSTEPSS (Chin et al., 2006). In the San Francisco Study, four percent of episodes of SE were due to metabolic causes, half of which failed to respond to first-line treatments. Metabolic causes were associated with poor outcome (severe neurologic deficit requiring full supportive care or death) in 65\% (Lowenstein and Alldredge, 1993).

Acute metabolic causes were the aetiology of SE in $11.5 \%$ with a mortality rate of $31 \%$ in one early study (Towne et al., 1994). Acute metabolic causes, although relatively rare as a cause of SE were responsible for a high proportion of treatment resistant SE with reported rates of $20 \%$ (Claassen et al., 2002), 22\% (Mayer, 2002) and 26\% in various series and a corresponding mortality rate of $25 \%$ in one study (Rossetti, et al. 2004). Indeed people with metabolic disorders presenting with SE were significantly more likely to require mechanical ventilation $(\mathrm{p}<0.0001)$ than people with $\mathrm{SE}$ due to other causes with a three-fold increase in mortality for those requiring mechanical ventilation compared with those who did not $(7.43 \%$ vs $2.22 \%$, odds ratio 2.79$)$ (Koubeissi et al., 2007).

\subsubsection{Cryptogenic status epilepticus}

Despite investigations, the aetiology of SE remains undetermined in many cases. In the Richmond study (DeLorenzo et al., 1996), approximately five percent of episodes of SE were classified as idiopathic with a mortality rate of $22 \%$. In the Rochester study (Hesdorffer et al., 1998) 17.5\% of episodes of SE were classified as idiopathic/cryptogenic, while the aetiology of SE was unknown in $13(8.7 \%)$ one of whom died in the Hessen study (Knake et al., 2001). In the NLSTEPSS (Chin et al., 2006), 21 (11.9\%) were classified as idiopathic or cryptogenic with $0 \%$ mortality, while 
in the San Francisco study (Lowenstein and Alldredge, 1993) the cause of SE was unknown in eight (5.2\%); five (62.5\%) had a good outcome. In a Dutch paediatric study the aetiology of SE was unknown in $110(31.8 \%), 100$ (91\%) of whom had a good outcome, seven (6.3\%) had associated morbidity and four died (Scholtes et al., 1994). In another study $14.2 \%$ of episodes of SE were classified as idiopathic with an associated mortality rate of $19.4 \%$ (Towne et al., 1994). The cause of SE was unknown in seven (8.4\%) episodes of SE in a German study none of whom developed treatment resistant SE $(p=0.017)$ (Holtkamp et al., 2005).

In a retrospective review of all people with idiopathic/cryptogenic seizures in Rochester over a 30 year period, 291 people with a first brief unprovoked seizure and 16 people with SE were identified. There were five deaths (all aged greater than 65) in those with SE. Compared with people with seizures, the adjusted relative risk for death in those with SE was 2.4 over ten years. This risk was more marked among those aged greater than 65 years $(\mathrm{RR}=5.1,95 \% \mathrm{CI} 1.6,15.7)$ and for those with SE who later developed epilepsy $(5 / 16,31.3 \%)(\mathrm{RR}=6.3,95 \%$ CI 1.5, 26.0). The SMR for SE was $2.6(95 \% \mathrm{CI}$ 0.8, 5.3) (Logroscino et al., 2008).

Auto-antibodies (VGKC-complex Abs,LGi1 and CASPR2-Abs, anti-NMDA Receptor Abs, GABA $\mathrm{A}$ Receptor Abs and Glycine Receptors Abs among others) have been increasingly recognised in the past few years as a potential cause of seizures and in particular encephalitis (Granerod et al., 2010; Dalmau et al., 2011; Vincent et al., 2011a; Vincent et al., 2011b). Autoimmune mediated encephalitis is more commonly associated with seizures than other causes of encephalitis (Granerod et al., 2010) with anti-NMDA receptor encephalitis (Dalmau et al., 2008; Irani et al., 2010) and LGi1 antibody limbic encephalitis (Irani et al., 2011) particularly associated with seizures. Autoimmune-mediated status epilepticus is, however, rare (Tan et al., 2010) even in the 
context of confirmed autoimmune mediated encephalitis with seizures. In a series of 100 people with anti-NMDA receptor encephalitis, $78 \%$ developed seizures during the course of the condition while eight people developed status epilepticus (which was refractory in six) (Dalmau et al., 2008). Nevertheless when status epilepticus does develop with autoimmune-mediated encephalitis, it is likely to be refractory to standard AED therapy and general anaesthesia. The possibility of an autoimmune-mediated aetiology of status epilepticus is important to consider, particularly in the setting of super-refractory status epilepticus (status epilepticus that continues or recurs 24 hours or more after the onset of anaesthetic therapy) (Shorvon and Ferlisi, 2011) as treatment with immunosuppression can sometimes result in a good outcome (Neligan et al., 2011c). 


\subsection{Other prognostic factors in SE}

Other factors such as seizure duration, EEG findings, patients' level of consciousness and age, are thought to influence prognosis in SE independent of aetiology. The evidence for the impact of these four factors on the prognosis of SE is now discussed.

\subsubsection{The duration of status epilepticus and its relation to outcome:}

It has been recognised since the 1970s that the longer the SE continues, the more difficult it is to control and the worse the outcome (Aicardi and Chervie, 1970). Any consideration of the impact of SE duration on prognosis needs to take into account the timing of initiation of treatment. It is well recognised that the longer the delay in initiating treatment inevitably the longer the duration of SE is likely to be. In a comparison of a group with prolonged seizures lasting 10 to 29 minutes versus a group with SE (defined as seizures for more than 30 minutes), $42 \%$ of seizures in the prolonged seizure group stopped spontaneously with $0 \%$ mortality while $58 \%$ of seizures were treated, with a $5.8 \%$ mortality rate $(\mathrm{P}<0.01)$. In contrast, only seven percent of episodes of SE stopped without treatment with no differences in mortality in the treated (19\%) and untreated groups (18\%) (DeLorenzo et al., 1999). Experimental studies have shown that as SE progresses, there are rapid changes to GABAergic receptor function, excessive NMDA receptor mediated transmission, mitochondrial changes and receptor trafficking (Chen and Wasterlain, 2006; Kapur and MacDonald, 1997; Shorvon et al., 2007) and these changes may be part of the reason for the increasingly resistant nature of the SE. The recognition of this point has lead to earlier and more aggressive treatment (and indeed a proposed change to the definition of SE) with an operational definition of SE of seizures lasting for more than five minutes being proposed (Lowenstein 1999). Such an aggressive approach to treatment is reflected in the two large clinical trails in SE. In the Veterans Affairs Cooperative Trial (Treiman et 
al., 1998) SE was defined as continuous seizure activity or two or more generalised seizures, without full recovery of consciousness between seizures of more than ten minutes duration. Similarly in the San Francisco study comparing out of hospital treatment of SE with lorazepam, diazepam and placebo, SE was defined as continuous or repeated seizure activity for more than five minutes without recovery of consciousness (Alldredge et al., 2001).

A representative early study (Towne et al.,1994) reported that a seizure duration of more than one hour was associated with a mortality rate of $32 \%$ compared with a mortality rate of $2.7 \%$ for seizure duration of less than one hour, a finding which remained statistically significant $(\mathrm{p}=0.0033)$ on multivariate analysis. Similarly a SE duration of more than one hour was associated with increased mortality $(39.4 \%$ vs $3 \%$, OR 2.41, $\mathrm{p}<0.0003$, univariate analysis) (Sagduyu et al., 1998). Others have made similar observations (Gulati et al., 2005). Treatment delay, defined as initiation of AED treatment 30 minutes after the onset of SE (and therefore seizure duration of one hour or more) is associated with poorer outcome on multivariate analysis (OR 3.51, $\mathrm{p}=0.04)$ (Hui et al., 2003).

In a prospective study of 645 people with SE comparing mortality in people with continuous and intermittent SE, adults but not children, with continuous SE had a significantly higher mortality than those with intermittent SE $(31.4 \%$ vs $19.6, \mathrm{p}<0.001)$ with a relative risk of mortality of $1.79(\mathrm{p}<0.04)$. After examining the role of ictal time versus SE duration and adjusting for age, aetiology and SE duration on multivariate analysis, however, ictal time was no longer predictive of mortality $(\mathrm{P}<0.60)$. In contrast, SE duration remained a highly significant predictor of mortality $(\mathrm{P}<0.001)$ indicating that it is SE duration rather than the duration that the person is actively seizing that is the primary determinant of mortality (Waterhouse et al., 1999).

The impact of SE duration and prognosis is summarised in Table 28. 


\begin{tabular}{|c|c|c|c|c|}
\hline Authors & Country & Number of cases & $\begin{array}{l}\text { Duration (for poor } \\
\text { outcome) }\end{array}$ & Study Design \\
\hline $\begin{array}{l}\text { Towne et al. } \\
\text { (1994) }\end{array}$ & $\overline{\mathrm{USA}}$ & 253 & $\begin{array}{l}>1 \text { hour } \\
32 \% \text { mortality } \\
<1 \text { hour } \\
2.7 \% \text { mortality } \\
\text { OR } 17.9\end{array}$ & $\begin{array}{l}\text { Retrospective } \\
\text { All aged }>16 \\
\text { years }\end{array}$ \\
\hline $\begin{array}{l}\text { Eriksson and } \\
\text { Koivikko (1997) }\end{array}$ & Finland & 65 & $\begin{array}{l}>2 \text { hours } \\
68.8 \% \text { morbidity }^{2} \\
<2 \text { hours } \\
32.7 \% \text { morbidity } \\
\mathrm{P}<0.025\end{array}$ & $\begin{array}{l}\text { Retrospective } \\
\text { Paediatric } \\
\text { study }\end{array}$ \\
\hline $\begin{array}{l}\text { Sagduyu et al } \\
\text { (1998) }\end{array}$ & Turkey & 66 & $\begin{array}{l}>1 \text { hour } \\
39.4 \% \text { mortality } \\
<1 \text { hour } \\
3 \% \text { mortality } \\
\text { OR } 2.41 \\
\mathrm{P}<0.003\end{array}$ & $\begin{array}{l}\text { Retrospective } \\
\text { All ages } \\
\text { (range 6-77 } \\
\text { years) }\end{array}$ \\
\hline $\begin{array}{l}\text { Gulati et al } \\
(2005)\end{array}$ & India & 30 & $\begin{array}{l}>45 \text { minutes } \\
100 \% \text { mortality } \\
<45 \text { minutes } \\
9.5 \% \text { mortality } \\
\mathrm{P}<0.001\end{array}$ & $\begin{array}{l}\text { Retrospective } \\
\text { Paediatric } \\
\text { Study }\end{array}$ \\
\hline $\begin{array}{l}\text { Dislane et al. } \\
\text { (2009) }\end{array}$ & USA & 119 & $\begin{array}{l}>10 \text { hours } \\
\text { OR } 4.3(95 \% \mathrm{CI} \\
1.21,15.3) \\
\mathrm{P}=0.0242 \\
>24 \text { hours } \\
\text { OR } 1.8(95 \% \mathrm{CI} \\
0.74,4.36) \\
\mathrm{P}=0.19(\mathrm{NS})\end{array}$ & $\begin{array}{l}\text { Retrospective } \\
\text { Adult } \\
\text { population } \\
\text { (range 24-96 } \\
\text { years) }\end{array}$ \\
\hline
\end{tabular}

Table 28: Duration and Status Epilepticus

${ }^{1}$ Poor outcome: morbidity and mortality. ${ }^{2}$ No reported deaths 
As was suggested by the classic French study of febrile SE in children in 1970 (Aicardi and Chervie, 1970), very long duration of SE is also associated with the development of neurological deficits, perhaps largely due to brain swelling, vascular effects or the underlying aetiology. More recent studies have shown that where new neurological signs develop due to SE, these appear after four hours of persistent SE (Drislane et al, 2009). In a review of 119 people with SE with a median duration of 48 hours, a duration of less than ten hours was associated with a better outcome (Proportion of people with a good outcome was $69 \%$ compared with $31 \%$ in people with a longer duration, $\mathrm{p}<0.05$ ) Once the aetiology of epilepsy, coma at presentation and generalised SE were corrected for on multivariate analysis, however, duration was no longer a significant predictor of outcome (Drislane et al., 2009). 


\subsubsection{EEG and prognosis of SE:}

While the EEG is an important tool in the diagnosis and management of SE, its role in terms of predicting prognosis is less established. Most attention has been paid to the presence of periodic epileptiform discharges (PEDs; or periodic lateralised epileptiform discharges - PLEDs). The association between PLED/PEDs and outcome however, does not seem to be strong, and furthermore PLEDs and PEDs are more likely to occur in certain aetiologies with poor prognosis (not least acute anoxic cerebral damage). One study found that if PEDs were present at any time during or after SE, outcome tended to be worse (44\% of the 18 patients with PEDs on at least one EEG vs $19 \%$ of the 32 without PEDs had a poor outcome) (Nei et al., 1999). There were no substantial differences in aetiologies between those with and without PEDs and structural lesions were not more associated with PEDs. The PEDs occurred at any time during and after SE and timing did not correlate with outcome. No other EEG characteristics (lateralised vs bilateral symmetric ictal EEG, discrete vs continuous ictal activity and post-ictal focal slowing) correlated with outcome and no predictable EEG sequence changes were found during SE. Another group found that the presence of burst suppression, ASIDs (After SE Ictal Discharges) and, less so, PLEDs were significantly associated with morbidity and mortality after controlling for aetiology on multivariate analysis (Jaitly et al., 1997). Others however have found that the outcome of SE was more related to age and aetiology than to any specific ictal EEG pattern (Garzon et al., 2001). In another study the presence of PLEDs was significantly associated with refractory SE compared with non-refractory SE on multivariate analysis $(42 \%$ vs $10 \%, \mathrm{p}=0.004)$ and the authors concluded that this reflected the longer duration of SE in these people (Mayer et al., 2002). In a study of 70 people prospectively followed, ictal discharges on EEG one 
hour post termination of SE (found in 15 of the 38 with seizure recurrence) were predictive of seizure recurrence within 24 hours (Kalita et al., 2006).

\begin{tabular}{|c|c|c|c|c|}
\hline Author & Number of Cases & $\begin{array}{l}\text { EEG features } \\
\text { during/after SE }\end{array}$ & Study Design & Prognosis \\
\hline \multirow{19}{*}{$\begin{array}{l}\text { Jaitly et al } \\
\text { (1997) }\end{array}$} & $\mathrm{N}=180$ & Burst & Prospective & \\
\hline & & suppression & Study & $78 \%$ Poor \\
\hline & & & & Outcome $^{1}$ \\
\hline & & & & (59\% mortality) \\
\hline & & & & OR 7.56 \\
\hline & & & & $\mathrm{P}<0.00005$ \\
\hline & & ASIDs & & $53 \%$ Poor \\
\hline & & & & Outcome \\
\hline & & & & (41\% mortality) \\
\hline & & & & OR 2.41 \\
\hline & & & & $\mathrm{P}<0.022$ \\
\hline & & PLEDs & & $53 \%$ Poor \\
\hline & & & & Outcome \\
\hline & & & & ( $40 \%$ mortality) \\
\hline & & & & OR 2.14 \\
\hline & & & & $\mathrm{P}<0.045$ \\
\hline & & Normal & & $0 \%$ Poor \\
\hline & & & & Outcome \\
\hline & & & & $\mathrm{P}<0.0008$ \\
\hline \multirow{7}{*}{$\begin{array}{l}\text { Nei et al. } \\
\text { (1999) }\end{array}$} & $\mathrm{N}=50$ & PEDs & Retrospective & $44 \%$ Poor \\
\hline & & & Study & Outcome in those \\
\hline & & & & with PEDs \\
\hline & & & & $19 \%$ Poor \\
\hline & & & & Outcome in those \\
\hline & & & & without PEDs \\
\hline & & & & $\mathrm{P}=0.053$ \\
\hline
\end{tabular}




\begin{tabular}{|c|c|c|c|c|}
\hline $\begin{array}{l}\text { Garzon et al. } \\
\text { (2001) }\end{array}$ & $\begin{array}{l}\mathrm{N}=55 \\
\text { (62 episodes of } \\
\mathrm{SE} \text { ) }\end{array}$ & $\begin{array}{l}\text { IES } \\
\text { MES } \\
\text { CID } \\
\text { PEDs (PLEDs } \\
\text { and PBEDs) }\end{array}$ & $\begin{array}{l}\text { Prospective } \\
\text { Study }\end{array}$ & $\begin{array}{l}\text { No pattern } \\
\text { independent of } \\
\text { aetiology was } \\
\text { predictive of } \\
\text { poor outcome }\end{array}$ \\
\hline $\begin{array}{l}\text { Mayer et al. } \\
(2002)\end{array}$ & $\begin{array}{l}\mathrm{N}=74 \\
\text { (83 episodes of } \\
\mathrm{SE})\end{array}$ & PLEDs & $\begin{array}{l}\text { Retrospective } \\
\text { Cohort Study }\end{array}$ & $\begin{array}{l}42 \% \text { developed } \\
\text { RSE compared } \\
\text { with } 10 \% \text { of } \\
\text { those without } \\
\text { PEDs } \\
\mathrm{P}<0.004\end{array}$ \\
\hline $\begin{array}{l}\text { Kalita et al. } \\
(2006)\end{array}$ & $\mathrm{N}=70$ & $\begin{array}{l}\text { 1-hour EEG } \\
\text { abnormality }\end{array}$ & $\begin{array}{l}\text { Prospective } \\
\text { Study }\end{array}$ & $\begin{array}{l}\text { Predictive of } \\
\text { seizure } \\
\text { recurrence within } \\
24 \text { hours }\end{array}$ \\
\hline $\begin{array}{l}\text { Lambrechtsen } \\
\text { and Buchhalter } \\
(2008)\end{array}$ & $\mathrm{N}=154$ & $\begin{array}{l}\text { Electrographic } \\
\text { seizures without } \\
\text { clinical } \\
\text { manifestations } \\
\text { Focal discharges }\end{array}$ & $\begin{array}{l}\text { Retrospective } \\
\text { Paediatric Study }\end{array}$ & $\begin{array}{l}31.7 \% \text { in those } \\
\text { with RSE vs } \\
13.8 \% \text { with ASE } \\
\mathrm{P}<0.027 \\
38.3 \% \text { in those } \\
\text { with RSE vs } \\
17 \% \text { with ASE } \\
\mathrm{P}<0.003\end{array}$ \\
\hline
\end{tabular}

Table 29: The EEG and the prognosis of Status Epilepticus

Glossary: ASE, aborted SE; ASIDs, after SE ictal discharges; CID, continuous ictal discharges; MES, merging EEG seizures; PEDs, periodic epileptiform discharges; PBEDs, periodic bilateral epileptiform discharges; PLEDs, periodic lateralised epileptiform discharges; RSE, refractory SE

${ }^{1}$ Poor Outcome: death or dependency at 30 days post SE on multivariate analysis controlling for aetiology. 


\subsubsection{GCS on admission:}

It is perhaps not surprising that a lower level of consciousness associated with SE at admission is a risk factor for poor prognosis. This reflects the severity of the SE and also of damage due to the underlying cause. This factor has been the subject of a number of investigations. One study found that when a patient presented with stupor or coma, there was higher mortality, more SE refractory to therapy, longer median hospital and a lower chance of return to baseline (Rossetti et al., 2006). Similarly another study demonstrated that the median Glasgow Coma Scale (GCS) score of people with treatment resistant SE was 6 compared with a median GCS of 7 for people with nonrefractory SE $(p=0.02)$, and that refractory SE was associated with reduced GCS at discharge compared with non-refractory SE ( $54 \%$ vs $28 \%, \mathrm{p}=0.02)$, increased median ICU stay ( 7.5 days vs 1 day, $\mathrm{p}<0.001)$, increased median hospital stay (32.5 vs 11 days, $\mathrm{p}<0.001)$ and increased mortality ( $23 \%$ vs $14 \%$; but this was not significant) on multivariate analysis (Mayer et al., 2002). The need for ventilation in SE (a marker of a decreased level of consciousness) has been shown to be associated with a higher mortality rate by others $(44.4 \%$ vs $12.5 \%, \mathrm{OR}=1.575, \mathrm{p}=0.004$ (Towne et al., 1994); Koubeissi and Alshekhlee, 2007).

Studies of level of consciousness and SE are shown in Table 30. 


\begin{tabular}{|c|c|c|c|c|}
\hline Reference & Number & $\begin{array}{l}\text { Marker of } \\
\text { Consciousness }\end{array}$ & Mortality & P-value \\
\hline $\begin{array}{l}\text { Sagduyu et al. } \\
1998\end{array}$ & 66 & $\begin{array}{l}\text { Need for ventilation } \\
(\mathrm{N}=18)\end{array}$ & $44.4 \%(\mathrm{~N}=8)$ & $\begin{array}{l}\text { OR } 1.58 \\
(95 \% \text { CI } 1.03, \\
2.41)\end{array}$ \\
\hline \multirow[t]{2}{*}{$\begin{array}{l}\text { Mayer et al. } \\
2002^{*}\end{array}$} & $\begin{array}{l}74(83 \\
\text { episodes) }\end{array}$ & $\begin{array}{l}\text { Median GCS score } \\
\text { on admission }\end{array}$ & & $\mathrm{P}=0.02$ \\
\hline & & $\begin{array}{l}6 \operatorname{RSE}(\mathrm{N}=26) \\
7 \text { Non-RSE }(\mathrm{N}=57)\end{array}$ & $\begin{array}{l}23 \%(\mathrm{~N}=6) \\
14 \%(\mathrm{~N}=8)\end{array}$ & NS \\
\hline \multirow[t]{5}{*}{$\begin{array}{l}\text { Rossetti et al. } \\
2006\end{array}$} & 96 & Alert $(\mathrm{N}=4)$ & $0 \%$ & $\begin{array}{l}\text { OR } 3.03 \\
(95 \% \text { CI } 1.05,\end{array}$ \\
\hline & & $\begin{array}{l}\text { Somnolent/Confused } \\
(\mathrm{N}=20)\end{array}$ & $5 \%(\mathrm{~N}=1)$ & 11.3) \\
\hline & & Stuporous $(\mathrm{N}=28)$ & $14.3 \%(\mathrm{~N}=4)$ & $\mathrm{P}=0.04 * *$ \\
\hline & & & $22.7 \%(\mathrm{~N}=10)$ & \\
\hline & & Comatose $(\mathrm{N}=44)$ & & \\
\hline $\begin{array}{l}\text { Koubeissi and } \\
\text { Alshekhlee }\end{array}$ & 11,580 & $\begin{array}{l}\text { Respiratory failure } \\
\text { necessitating }\end{array}$ & $7.4 \%(\mathrm{~N}=202)$ & $\begin{array}{l}\text { OR } 2.79 \\
(95 \% \mathrm{CI}\end{array}$ \\
\hline \multirow[t]{2}{*}{2007} & & $\begin{array}{l}\text { intubation } \\
(\mathrm{N}=2,784)\end{array}$ & & $2.18,3.59)$ \\
\hline & & & & $\mathrm{P}<0.0001$ \\
\hline
\end{tabular}

Table 30: Level of consciousness and Status Epilepticus

*Refractory SE (RSE) was defined as SE lasting $>1$ hour and failure of 2 AEDs. Lower GCS score was significantly associated with RSE but not increased mortality

**Increased mortality associated with decreased level of consciousness 


\subsubsection{Age:}

\subsubsection{Status epilepticus in children:}

The range of aetiologies of status varies considerably with age, and perhaps largely for this reason age is a major determinant of prognosis. The extent to which age per se renders an individual more or less susceptible to adverse outcomes is less clear, but it is frequently stated that SE in very young children has less risk than SE in older children or adults. For instance, in an old study of 193 children with SE (mean age five years), causes were classified as idiopathic in 46 , febrile in 46 , acute symptomatic in 45 and progressive neurologic in 11. All seven deaths occurred in the progressive neurologic group and of the 186 survivors, new neurologic deficits were found in $17(9.1 \%)$. Fifteen of the 17 sequelae occurred in children with acute or progressive neurologic deficits. Of the 125 surviving children with no previous history of seizures, $30 \%$ subsequently had a further unprovoked seizure (Maytal et al., 1989).

Febrile illness, with or without infection, is a predominant cause of SE in young children, accounting for 32\% of all episodes of SE in the NLSTEPSS (Chin et al., 2006).

A review of the published literature over the last 15 years found that febrile SE was noted to have a low mortality (14 out of 876 people with febrile SE; $1.6 \%$ ) and this has fallen significantly from the early 1970 s probably due to early and aggressive treatment of status epilepticus (Chungath and Shorvon, 2008). A cohort of 180 children aged one to ten years with febrile SE followed prospectively over ten years, were compared with 244 children with their first febrile seizure. There were no deaths and no cases of cognitive impairment (Shinnar et al., 2001). 
In population studies, the occurrence of SE in children does not seem to be associated with poorer overall mortality or prognosis. In the Dutch study of 494 children with newly diagnosed epilepsy, followed prospectively for five years, SE occurred in 47 $(9.5 \%)$ but neither terminal remission nor mortality was significantly worse in the children with SE (Stroink et al., 2007). Similarly in a cohort of 150 children followed over 33 to 36 years, $41(27 \%)$ had an episode of SE of whom $22(56 \%)$ had two or more episodes. The occurrence of SE did not alter the mortality rates and had only a modest impact on the probability of attaining remission (Eriksson and Koivikko 1997). In a study of 65 children from Finland permanent neurological sequelae secondary to SE occurred in $15 \%$ and were associated with SE duration of greater than two hours. There were no SE related deaths (Sillanpaa and Shinnar 2002).

Other studies from around the world have given varying mortality figures of SE in children: Japan (234 children; 19.2\% poor outcome and 4\% mortality (Maegaki et al., 2005)); Iran (135 children, mortality 12.6\%, morbidity 27.3\% (Asadi-Pooya and Poordast, 2005)); Korea (189 children, mortality 3\%, morbidity 33\% (Kang et al., 2005)); India (30 people, mortality 30\% (Gulati et al., 2005)); Turkey (83 children, mortality 7.2\% (Karasalihoglu et al., 2003)); Thailand (32 children, mortality 6.3\%, morbidity 56.3\% (Visudtibhan et al., 2006)); Kenya (388 children, 15.2\% mortality, 11.9\% morbidity (Sadarangani et al., 2008)) and Hong Kong (37 children, mortality $11 \%$, morbidity $27 \%$ (Kwong et al., 2004)).

Other factors also have an impact on the relationship between age and outcome, and these were explored in a retrospective study of SE in 154 children (Lambrechtsen and Buchhalter, 2008). Sixty (39\%) had treatment resistant SE which was significantly associated with: a family history of seizures, higher seizure frequency, higher number of maintenance AEDs, non-convulsive SE and focal or electrographic seizures on initial 
EEG. Predictors for poor outcome in both children with treatment-responsive and treatment -resistant SE were long seizure duration $(\mathrm{p}<0.001)$, acute symptomatic aetiology $(\mathrm{p}=0.04)$ (which included causes such as acute metabolic disorders $(32.5 \%)$, encephalitis (25\%), acute brain trauma (12.5\%), stroke (12.5\%), hypoxia-ischaemic encephalopathy (10\%)and AED non-compliance $(9 \%)$ ), non-convulsive $\mathrm{SE}(\mathrm{p}=0.001)$ and age at admission $<5$ years at admission $(\mathrm{p}=0.05)$ (Lambrechtsen and Buchhalter, 2008).

Other studies (Maytal et al., 1989) have suggested that if SE fails to respond to initial therapy, the prognosis is poor with high mortality and morbidity. In a study of the outcome of treatment resistant SE in 22 children, the mortality rate was $32 \%$ and subsequent seizures occurred in 53\% of survivors. Prognosis was largely related to aetiology, with the worst prognosis in the acute symptomatic group where out of eight children, three died and five developed a neurological deficit or seizures with none returning to baseline (all presumed or confirmed encephalitis). Other causes associated with a poor outcome were CNS lymphoproliferative disease, brainstem neoplasm, Alpers disease, MCAD disease and previous haemorrhage (Sahin et al., 2001).

\subsubsection{Status epilepticus in adults}

The outcome of SE in adults is poorer than in children and is particularly bad in those over 60 years of age as demonstrated in the Richmond study where children had a mean mortality rate of three percent, young adults (aged 15-59) a 13\% mortality rate and people aged over 60 years a 38\% mortality rate (DeLorenzo et al., 1996). The major reason seems again to be the underlying aetiology, with most episodes of convulsive SE in the elderly due to acute stroke or anoxic brain damage. This has been confirmed in various studies. The mortality rate of SE in the elderly was found to be $30 \%$ (342 patients) (Claassen et al., 2002) and 29\% with an odds ratio of death of 5.41 at age over 
65 years compared with people aged less than 65 years (Rossetti et al., 2006). Age over 45 years at the time of SE was significantly associated with mortality $(77.7 \%$ vs $12.2 \%$; $\mathrm{OR}=2.88, \mathrm{p}<0.011)$ (Sagduya et al., 1998). In an older study the mortality rate was found to increase with age with a mortality rate of $12.5 \%$ in people aged 16 to 30 years, $15.2 \%$ in people aged 30 to 60 years and a mortality rate of $32.3 \%$ in people aged over 60 years with SE (Towne et al., 1994).

In a multiple logistic regression analysis of mortality in SE in a study of 83 people with $\mathrm{SE}$, increased age was found to be predictive of death in SE (mean age of people who died was 78 years vs 59 years with a good functional outcome; Odds Ratio 1.1, p=0.02) (Claassen et al., 2002). Similarly mortality rates in SE were shown to increase with increasing age in a large in-hospital series with mortality rates of $0.7 \%$ for those aged ten years or younger, $1.3 \%$ (ages 11-20), 1.2\% (ages $21-30), 2.1 \%$ (ages $31-40), 2.6 \%$ ( ages $41-50$ ), $5.1 \%$ (ages $51-60$ ), 6.8\% (ages $61-70$ ), $8.1 \%$ (ages $71-80$ ) and $10.2 \%$ for those aged over 80 years (Koubeissi and Alshekhlee, 2007).

Studies of age and status epilepticus are shown in Table 31. 


\begin{tabular}{|c|c|c|c|c|}
\hline Reference & Country & Number of Cases & Mortality/Morbidity & Study Design \\
\hline $\begin{array}{l}\text { Maytal et al. } \\
1989\end{array}$ & USA & 193 & $\begin{array}{l}3.6 \%(\mathrm{~N}=7) \text { mortality } \\
8.8 \%(\mathrm{~N}=17) \text { morbidity }\end{array}$ & $\begin{array}{l}\text { Paediatric } \\
\text { Prospective/ } \\
\text { Retrospective }\end{array}$ \\
\hline $\begin{array}{l}\text { Scholtes et al. } \\
1994\end{array}$ & Holland & 346 & $\begin{array}{l}11.0 \%(\mathrm{~N}=38) \\
\text { mortality } \\
13.0 \%(\mathrm{~N}=45) \\
\text { morbidity }\end{array}$ & $\begin{array}{l}\text { All aged } \geq 15 \\
\text { Retrospective }\end{array}$ \\
\hline $\begin{array}{l}\text { Towne et al. } \\
\text { 1994* }\end{array}$ & USA & 253 & $\begin{array}{l}23.3 \%(\mathrm{~N}=60) \\
12.5 \% 16-30 \text { years } \\
15.2 \% 30-60 \text { years } \\
32.3 \%>60 \text { years }\end{array}$ & $\begin{array}{l}\text { All aged } \geq 16 \\
\text { Retrospective }\end{array}$ \\
\hline $\begin{array}{l}\text { DeLorenzo et al. } \\
1996\end{array}$ & USA & 166 & $\begin{array}{l}22 \% \text { mortality overall } \\
3 \%(\text { children }) \\
38 \%(\text { age }>60)\end{array}$ & $\begin{array}{l}\text { All aged }>1 \\
\text { month } \\
\text { Prospective }\end{array}$ \\
\hline $\begin{array}{l}\text { Eriksson \& } \\
\text { Koivikko } \\
1997\end{array}$ & Finland & 65 & $\begin{array}{l}0 \% \text { mortality } \\
15 \% \text { (neurological } \\
\text { morbidity) } \\
23 \% \text { (de novo epilepsy) }\end{array}$ & $\begin{array}{l}\text { Paediatric } \\
\text { Retrospective } \\
\text { Mean follow-up } \\
3.6 \text { years }\end{array}$ \\
\hline $\begin{array}{l}\text { Sillanpaa \& } \\
\text { Shinnar } 2002 * *\end{array}$ & Finland & 41 & $17 \%(\mathrm{~N}=7)$ & $\begin{array}{l}\text { Paediatric } \\
\text { Prospective study } \\
\text { of } 150 \text { children } \\
\text { with epilepsy over } \\
23-26 \text { years }\end{array}$ \\
\hline $\begin{array}{l}\text { Rossetti et al. } \\
2006\end{array}$ & USA & 96 & $\begin{array}{l}15.6 \%(\mathrm{~N}=15) \\
\text { Mortality } \\
\text { Age } \geq 65 \\
\text { OR } 5.41(1.3,25.5) \\
\mathrm{P}=0.02\end{array}$ & $\begin{array}{l}\text { Retrospective } \\
\text { All aged } \geq 16 \\
1^{\text {st }} \text { episode of SE }\end{array}$ \\
\hline $\begin{array}{l}\text { Stroink et al. } \\
2007\end{array}$ & Holland & 47 & $4.3 \%(\mathrm{~N}=2)$ & $\begin{array}{l}\text { Paediatric } \\
\text { Prospective } 5 \\
\text { follow-up }\end{array}$ \\
\hline $\begin{array}{l}\text { Koubeissi \& } \\
\text { Alshekhlee } 2007\end{array}$ & USA & 11,580 & $\begin{array}{l}3.45 \%(\mathrm{~N}=399) \\
0.67 \% \text { Age } \leq 10 \\
10.15 \% \text { Age }>80\end{array}$ & $\begin{array}{l}\text { All ages } \\
\text { Retrospective }\end{array}$ \\
\hline
\end{tabular}

Table 31: Age and Status Epilepticus 
*Age significant predictor of mortality on multivariate analysis $(\mathrm{P}=0.0155)$

**No significant difference in mortality in children with $(\mathrm{N}=41)$ and without SE $(\mathrm{N}=109)$ 


\subsection{Outcome measures in status epilepticus}

In discussing the prognosis of $\mathrm{SE}$, the outcome measures which are usually considered are mortality and three measures of morbidity (functional deficit, neuro-cognitive deficit and the risk of ongoing epilepsy). Each of these outcomes following SE, based on the literature, are now discussed.

\subsubsection{Mortality and status epilepticus}

The studies mentioned earlier were concerned largely with mortality. Formal measures of mortality after SE can take several forms. The most commonly employed is the case fatality (CF), the proportion of people in the cohort dying, and the 30 day mortality rate is taken as standard (Logroscino et al., 2005). CF was 19\% in the Minnesota study (Hesdorffer et al., 1998) and 22\% in the Richmond study (DeLorenzo et al., 1996), while the CF figure for the EPISTAR study (Coeytaux et al., 2000) was 7.6\% (with the exclusion of post-anoxic patients) and 9.3\% in the Hessen study (Knake et al., 2001). By contrast the 30 day CF for the Bologna study was 33\% (Vignatelli et al., 2003) (excluding post-anoxic patients) while the 30 day CF in another study from a different area in Italy (Lugo di Romagna)(Vignatelli et al., 2005) was only seven percent. The authors emphasised the impact of treatment and different regional treatment protocols in the acute management of SE for the disparity in the CF rates between the two studies (Vignatelli et al., 2008). As noted, the outcome and mortality rates for paediatric SE are better than for adults. In a recent review of the outcome of paediatric convulsive SE the short-term mortality was between 2.7 and 5.5\% (Raspall-Chaure et al., 2006). While the 30-day CF overall in the California study (Wu et al., 2002) was 10.7\%, the childhood CF associated with GCSE was only $1.4 \%$, the lowest reported to date. 
One group looked at the short-term mortality (1997) and long-term mortality (2002) in a cohort of 201 people with a first episode of afebrile SE identified between 1965 and 1984 in Rochester. The CF within the first 30 days was 19\% (38 deaths) with 34 (89\%) deaths occurring in people with acute symptomatic SE (anoxic encephalopathy $38 \%$, cerebrovascular disease $21 \%$ ) while four (11\%) occurred in people with unprovoked SE. Age over 65 years was associated with a relative risk of short-term mortality of 18.4 (Logroscino et al., 1997). At ten years, the cumulative mortality among 30-day survivors was $43 \%$ with an SMR at ten years of 2.8 (95\% CI, 2.1, 3.5). The SMR however, was not increased for those with cryptogenic/idiopathic SE (SMR=1.1; 95\% CI 0.5, 2.3). The long-term risk for mortality was increased for SE at least 24 hours in duration vs $\mathrm{SE}$ of less than two hours ( $\mathrm{RR}=2.3 ; 95 \% \mathrm{CI} 1.1,5.1)$, acute symptomatic aetiology vs idiopathic/cryptogenic $(\mathrm{RR}=2.2,95 \% \mathrm{CI} 1.0,5.1)$ and myoclonic vs generalised convulsive $\mathrm{SE}(\mathrm{RR}=4.0 ; 95 \% \mathrm{CI} 1.3,13)$. Based on these findings it was suggested that SE alone does not modify long-term mortality (Logroscino et al., 2002).

\subsubsection{Morbidity - neurological deficit and status epilepticus}

In the study from San Francisco of 154 people with SE (Lowenstein and Alldredge, 1993), 15 (9.7\%) had severe neurological deficits requiring long-term hospitalisation or full supportive care. In the Dutch study of 346 children, 45 (13\%) had resultant morbidity (15 (33.3\%) had cognitive deterioration, $13(28.9 \%)$ had paresis, three had impairment of consciousness, two had aphasia and one had ataxia). The sequelae however, were felt to be directly attributable to the SE in only $27 \%$ (the deficits were due to the underlying cause in $53 \%$ of children, with the main contributing factor undetermined in the remaining 20\%) (Scholtes et al., 1994). 
In children morbidity following SE remains low in the absence of acute symptomatic or progressive cause with less than $15 \%$ developing new neurological deficits as a result of SE (Raspall-Chaure et al., 2006). In an older study neurological deficits occurred in 17 (9.1\%) of survivors of SE of whom 15 had acute or progressive insults and only two of the 137 with other causes sustained any new deficits $(\mathrm{p}<0.001)$ (Maytal et al., 1989). Others have reported morbidity rates following SE of 15\% (Eriksson and Koivikko, 1997) and 15.4\% (Maegaki et al., 2005). One study, in contrast to the overall trend, found significant morbidity in children three months after an episode of SE with $79 \%$ of children being abnormal neurologically and 34\% demonstrating neuro-developmental deterioration at follow-up with predictors being aetiology (non-idiopathic or nonfebrile), SE at twelve months or younger and abnormal neuro-imaging (Barnard and Wirrell, 1999).

Morbidity rates were retrospectively examined in the Rochester cohort (Cascino et al., 1998). In the 146 people surviving 30 days (20.6\% mortality) (Logroscino et al., 1997) morbidity attributable to SE was noted in five people (3.4\%) including three with hemiparesis and encephalopathy, one with LD and one with aphasia. All morbidity occurred in people with either acute symptomatic (four) or remote symptomatic (one) aetiologies. The authors concluded that morbidity related to SE was uncommon and related to the underlying cause (Cascino et al., 1998).

Morbidity and mortality rates appear to be higher in people who develop treatment resistant SE. People with non treatment resistant SE and morbidity were more likely to return to baseline (50\%) compared with people with treatment resistant SE and resultant morbidity $(31 \%)(p=0.04)$. Over half $(54 \%)$ of people with treatment resistant SE had functional deterioration compared with $28 \%$ of people with treatment responsive SE 
$(p=0.02)$ (Rossetti et al., 2005). In children treatment resistant SE is similarly associated with higher morbidity. None of the seven children with morbidity in a series of 22 children with treatment resistant SE returned to normal (Sahin et al., 2001) while in another study the number of children who returned to baseline was significantly less in children with treatment resistant SE than in children with treatment responsive SE (66.6\% vs $82.9 \% ; \mathrm{p}=0.05)$ and they had a higher risk of developing a new deficit (71.4\% vs 55.1\%; $\mathrm{p}=0.0001)$ (Lambrechtsen and Buchhalter, 2008).

\subsubsection{Morbidity - neuro-cognitive sequelae of status epilepticus}

The question as to whether or not seizures and in particular SE are risk factors for cognitive dysfunction has been debated for many years. In an early review of the literature of studies of cognitive impairment in relationship to SE, nineteen studies were analysed and the following conclusions were drawn: most studies involved children, did not use any type of formal psychological assessment and were retrospective. The older studies tended to report greater losses in cognitive abilities after SE than more recent studies. The authors concluded that almost every study attributed at least some negative effects to SE per se (Dodrill and Wilensky, 1990). Certainly it is common clinical experience to see that memory and other cognitive functions are impaired immediately after an episode of severe SE, but these may often improve over ensuing months.

Formal studies, in the literature are sparse. Most have found no evidence of persistent cognitive deficits (Adachi et al., 2005; Dodrill and Wilensky, 1990; Dunn, 1988; Ellenberg et al., 1986; Ellenberg and Nelson, 1978).

In the UK national cohort study 37 children with a history of prolonged seizures were prospectively followed up (19 had lengthy febrile seizures and 18 had SE). of the 35 survivors, 33 underwent intellectual evaluations, which were normal in 23 and abnormal 
in ten. Eight of these children, however, had preceding developmental or neurological abnormality, implying that the cognitive outcome is more determined by the underlying cause (Verity et al., 1993). There is an anecdotal report in the literature of a young woman with progressive cognitive and neurological deficits during a parietal lobe SE, who had complete normalisation of cognitive and neurological function one year after seizure remission (Van Paesschen et al., 2007).

\subsubsection{Morbidity - the risk of epilepsy after status epilepticus}

There is a great deal of unequivocal experimental evidence in animal models of SE showing that an episode of SE will lead to continuing epilepsy, and it seems likely that a similar risk occurs in humans. As in other aspects of prognosis, however, it is difficult to differentiate the risk of ongoing epilepsy following SE itself from that of the underlying condition.

In the review of outcome of paediatric SE, the risk of subsequent epilepsy following an episode of SE ranged from 13 to 74\% (Raspall-Chaure et al., 2006). In a British cohort study 14 (82\%) children developed afebrile seizures after SE compared with only four (21\%) children with prolonged febrile seizures. Over one third (36\%) of children with no history of seizures developed epilepsy following SE and 25\% developed refractory epilepsy with 50\% having recurrent SE (Barnard and Wirrell 1990). During a mean follow-up of 3.6 years, $23 \%$ of children developed epilepsy following SE in a Finnish study (Eriksson and Koivikko 1997) while 30\% (37) had subsequent unprovoked seizures in an earlier paediatric study (Maytal et al., 1989). In a prospective study of 95 children followed up for a mean of 29 months from the time of a first episode of SE, the overall risk of recurrence of SE was $17 \%$ (16 children), with SE recurring primarily in neurologically abnormal children $(88 \%(14), \mathrm{p}<0.001)$ and in all five children with 
multiple episodes $(\mathrm{p}<0.001)$ (Shinnar et al., 1992). The risk of developing epilepsy following a single seizure was 44\% (Shinnar et al., 1996) implying that SE by itself does not significantly add to the risk of subsequent epilepsy in children.

One study investigated the impact of SE in the development of subsequent epilepsy. At ten years follow-up, the risk of unprovoked seizure was $41 \%$ for people with acute symptomatic SE compared with $13 \%$ for people with a single acute symptomatic seizure without SE. After controlling for cause of acute symptomatic seizure, age and sex, acute symptomatic SE was associated with a 3.3-fold increase in risk for a first unprovoked seizure compared with those with acute symptomatic seizure only. Moreover the risk of a subsequent unprovoked seizure was increased 18.8-fold following SE with anoxic encephalopathy, 7.1-fold for structural causes of SE and 3.6fold with metabolic SE. This study provides support to the belief that SE in adults is a significant factor in the development of subsequent epilepsy (Hesdorffer et al., 1998b).

The risk of epilepsy following SE in children and adults seems also determined by the severity of the episode of SE. In one study, symptomatic epilepsy developed more often in those with treatment resistant SE (87.5\%) than in people with treatment responsive SE $(22.2 \% ; \mathrm{p}<0.05)($ Holtkamp et al., 2005). This trend is also seen in children where in one study of treatment resistant SE all surviving children developed intractable epilepsy (Sahin et al., 2001) while in another study the risk of subsequent epilepsy was significantly higher for those with treatment resistant SE (30.6\% vs $16 \%$; $=0.004)$ (Lambrechtsen and Buchhalter 2008). 


\section{Chapter 8: Discussion}

In the discussion several of the studies are discussed together, where applicable, in relation to different prognostic themes: epilepsy and mortality (studies 1 and 2), seizure prognosis of people with chronic epilepsy (studies 3 and 4) and a prognostic model of epilepsy (studies 2-4). The prognosis of febrile seizures (study 2) and the prognosis of status epilepticus (study 5) are discussed separately.

\subsection{Epilepsy and mortality (Studies 1\&2)}

An attempt was made to address two issues with regard to the risk of premature mortality in people with epilepsy 1) how SMRs change during the course of the condition, with particular reference to the long-term mortality data from the NGPSE, and 2) how reported mortality rates have changed over time.

\subsubsection{SMRs (Study 1)}

SMRs compare the mortality rates in people with epilepsy with that of the population from which they derive and hence give some indication of the premature mortality experienced by them. As previously discussed (Section 1.6.1, Page 55), direct comparison of SMRs derived from different populations is problematic, particularly between populations of different age groups with many studies having an overrepresentation of young people. This results in proportionally higher SMRs due to the lower expected death rate in children and young adults (Camfield et al., 2002). Indeed it has been suggested that these potential differences may even invalidate direct 
comparison of SMRs derived from distinct populations (Logroscino and Hesdorffer, 2005).

In examining reported SMRs in prevalent cohorts, it needs to be emphasised that prevalence studies generally under-represent people with milder forms of epilepsy whilst simultaneously missing some people who may have died previously for whatever reason. It is clear, therefore, that results from incident cohorts provide a more accurate picture. Indeed, so great are the potential inaccuracies of prevalence studies that these cannot be considered useful and they are not considered further. Currently, there are few incident cohorts available for comparison. 


\subsubsection{The long-term risk of premature mortality - evidence from the NGPSE} (Study 2)

There have been few population-based studies of mortality in people with epilepsy with more than ten years follow-up. This analysis extends follow-up of the NGPSE considerably, increasing mean follow-up to over 18 years and the person-years by twothirds, from the last analysis (Lhatoo et al., 2001). Overall there were no major differences from the previous analyses; extra follow-up has, however, increased the precision of the estimates and narrowed the CIs.

This analysis is in line with previous reports arising from the NGPSE cohort using information available at the time of the initial ( 6 month) classification by a review panel. The designation of some cases as possible/probable epilepsy had the advantage at the time of allowing the inclusion in the study of some cases which would otherwise have been excluded due to a lack of a definitive diagnosis of epilepsy. It was also in line with the original study design which was intended to be over-inclusive to allow GPs to refer people who they considered might have a diagnosis of possible epilepsy.

A pragmatic decision was taken at the time that changing diagnostic categories according to information acquired later in the follow-up period was not a sound approach to the analysis of longitudinal data. This is standard epidemiological practice in line with other longitudinal prospective cohort studies. No attempt has been made to re-assess the aetiology of epilepsy in those classified as having idiopathic/cryptogenic epilepsy or to re-evaluate the original diagnostic categories, in part because such reclassification would be incomplete (in people with a short course of active epilepsy in whom no further investigations would have been carried out or in people who died early 
in the course of their epilepsy) and therefore subject to bias. Similarly no analysis with regard to co-morbidities was possible due to incomplete data, in particular as specific inquiry into co-morbidities was not permitted in later follow-up due to recently introduced legal restrictions. Consequently all people remain in the same categories in which they were classified at six months after the index seizure. Because of the diagnostic uncertainty in those categorised as having possible/probable epilepsy, we have continued to present separate SMRs for those with definite and probable/possible epilepsy. The overall SMR for those with possible/probable epilepsy was significantly elevated but less so than in those with definite epilepsy.

Mortality was elevated overall for all aetiological subgroups of people classified as having definite epilepsy. It is well recognised that people with remote symptomatic epilepsy or epilepsy associated with a congenital deficit have higher SMRs and indeed remote symptomatic aetiology is the major determinant of mortality in children (Camfield et al., 2002). Some studies, however, have not reported an increased SMR in people with idiopathic or cryptogenic epilepsy (Lindsten et al., 2000; Olafsson et al., 1998) although one study did (Hauser et al., 1980). The SMR of those with idiopathic /cryptogenic epilepsy in our cohort was significantly elevated for the last ten years of follow-up, which was not seen previously (Cockerell et al., 1994; Lhatoo et al., 2001).

Mortality was most marked in the younger age groups (aged less than 60 years) for those with definite and possible epilepsy as seen previously (Lhatoo et al., 2001). The cohort included 83 people with acute symptomatic seizures with an overall SMR of 3.2 (95\% CI 2.4, 4.3) which has been relatively constant throughout follow-up. A recent study reported that people with acute symptomatic seizures were nine times more likely to die within the first thirty days than those with a first unprovoked seizure. In contrast 
there was no difference in mortality at ten years in those who survived 30 days when adjusted for age and aetiology (Hesdorffer et al., 2009). In our study, the SMR remained significantly elevated $(2.5,95 \%$ CI $1.7,3.5)$ in those who survived the first year.

The study records of all 83 people originally classified as having acute symptomatic seizures were re-examined and re-classified according to the new definition of acute symptomatic seizures (a seizure occurring usually within seven days of an acute CNS insult (Beghi et al., 2010)). Sixty people remained classified as having acute symptomatic seizures. It was found, however, that changing the timing of the seizure from within three months to within one week of the precipitating cause in our classification did not make any difference to the overall mortality with the reported SMRs being virtually identical (Table 11, Page 111).

The overall SMRs for those with definite and possible seizures remained significantly raised throughout follow-up. There is a weak suggestion that there is a late increase (20-25 years) in the SMR. While this late increase is not significant in the NGPSE, premature mortality in the cohort does not appear to be decreasing. To investigate this possible late increase in mortality, the study records of the people who died in the last 10 years of follow-up were examined. Of these $71 \%$ people were seizure free for the previous five years at last follow-up and 39\% were off AEDs. The fact that the majority of those who died remained on AEDs raises the possibility that some died as a result of AED non-compliance which has been suggested to increase risk of premature mortality (Faught et al., 2008) in people with previously well controlled seizures. This is, however, unlikely to be the explanation in the majority of our cases as the trend of an increasing proportion of the cohort being in terminal remission has continued with longer follow-up (Cockerell et al., 1997; Neligan et al., 2010b). 
It has been suggested that AEDs are possibly associated with an increased risk of cancer (Olsen et al., 1989; Nilsson et al., 1997; Lamminpaa et al., 2002; Singh et al., 2007) although others have not found any definitive evidence for such an association (White et al., 1979; Adelow et al., 2006). We found, however, that cancer-related deaths were highest in the first five years (and therefore possibly the cause of seizures) and again in the final years of follow-up. The reason for the modest increase in later years is unclear, but the possibilities include the prolonged use of AEDs or common genetic predispositions; these hypotheses have not yet been tested.

Another possible explanation for the higher mortality rates in people with epilepsy is the interplay between epilepsy and socio-economic status. It has been shown that the incidence of epilepsy appears to increase with socio-economic deprivation (Heaney et al., 2002). Similarly it has been suggested that socio-economic deprivation is associated with a lower life-expectancy even in developed countries with universal access to health care. (De Vogli et al., 2005). Lower economic status may lead to poor treatment access and disparities in health care as a result of rural isolation, ethnicity and lack of public and physician knowledge of modern approaches to epilepsy care and this may lead to an increased risk of premature mortality (Theodore et al., 2006).

At this stage, any explanation of why there is a possible late increase in mortality in people with a history of seizures (or why there is no decrease in mortality despite an increasing proportion of people who are seizure free) can only be speculative. It does, however, raise the possibility that a predisposition to seizures (with or without clearly identified aetiology) may be but one manifestation of a polygenic phenotype with other associated conditions which may also predispose to premature mortality. This may, in 
part, explain why we did not find a significant difference in the mortality in people with acute symptomatic compared with those with remote symptomatic seizures.

The suggestion of a persistently elevated risk of premature mortality throughout followup raises a dilemma when counselling people with epilepsy as to what they should be told of an increased risk of premature death irrespective of seizure control.

In the NGPSE, the causes of death were based on those listed on the death certificate which also included other conditions or co-morbidities which were believed to have contributed to, but which were not the primary cause of, death. No other clinical or para-clinical information about the circumstances in which the death occurred was used. Only in a small number of cases where there was doubt about the primary cause or circumstances of the death was a post-mortem performed which was at the discretion of the local coroner. Despite the well documented problems of the use of death certificates in people with epilepsy (Bell et al., 2004), death certificates form the basis for mortality data in population studies and were the source of cause-specific mortality in the two other long-term population-based studies in people with epilepsy (Hauser et al., 1980; Rafnsson et al., 2001).

One might expect a decrease in SMR with prolonged follow-up in a cohort of people largely seizure free, yet we and others have not seen this (Hauser et al., 1980). We thus looked at specific causes of death to identify possible reasons for this. The most common causes of death for those with definite or possible epilepsy, as recorded in the death certificates, were cancers (including lung cancer), cerebrovascular disease, pneumonia and ischaemic heart disease. Pneumonia was common with a significantly increased SMR in the group with definite and possible epilepsy at all points of followup (Table 13, Page 116) and appearing to increase at 15-20 and 20-25 years follow-up. 
Pneumonia has long been recognised as a common cause of premature mortality in people with epilepsy (Munson, 1910) and this has been replicated in other, more recent, studies (Hauser et al., 1980; Lindsten et al., 2000; Nilsson et al., 1997); the reason for this increase is currently unclear. Intuitive suggestions that this may be associated with aspiration as a result of seizures have not been tested and this would not, in any case, apply to people in remission. In some cases, pneumonia with fever and administration of antibiotic therapy could have precipitated a seizure contributing to mortality with only pneumonia recorded on the death certificate. Similarly it has been shown that generalised tonic-clonic seizures can lead to neurogenic pulmonary oedema with symptoms resembling pneumonia (Baumann et al., 2007) with the result that the primary cause of death could be mistakenly recorded as pneumonia instead of epilepsy. Another possible explanation for the high prevalence of pneumonia is the recently postulated theory that inflammatory mechanisms are involved in the pathogenesis of epilepsy with the result that chronic epilepsy may lead to relative suppression of the immune system with a subsequent increased risk of infections such as pneumonia (Vezzani and Granata, 2005; Najjar et al., 2008; Bauer et al., 2009). None of the above possible theories, however, explains why pneumonia continues to feature so predominantly in the later stages of follow-up when the majority of people have entered terminal remission.

The SMR for cerebrovascular disease was significantly elevated for the first fifteen years of follow-up but not thereafter. As expected, the SMRs for all cancers and for cancers excluding brain tumours were significantly elevated in the first five years, decreasing thereafter but were both again significantly elevated at 20-25 years followup. The SMR for lung cancer was significantly elevated only during the first five years although numbers were inevitably smaller. The SMR was significantly elevated for 
ischaemic heart disease (SMR 3.34, 95\% CI 1.6, 7.0) for the first time at 20-25 years, although the confidence intervals are wide. This was also associated with an increased SMR in a hospital-based study (Nilsson et al., 1997) and in population-based study but only for those aged $<65$ years old (Annegers et al., 1984).

\subsubsection{Temporal trends in mortality (Study 1)}

As we have confirmed, the risk of premature mortality is significantly higher than that of the general population in the initial one to two years following seizure onset and this risk decreases thereafter with the SMR often falling to rates comparable with those of the general population (Cockerell et al., 1994; Hauser et al., 1980; Loiseau et al., 1999; Neligan et al., 2010a). Much of this initial risk is related to the underlying aetiology and seizure type as seen in the Gironde study (Loiseau et al., 1999) and the Canadian paediatric study (Camfield et al., 2002).

There is a suggestion, however, from several studies and possibly in the current investigation of the NGPSE that there is a subsequent increase in mortality ratio after more prolonged follow-up: 10 years (Camfield et al., 2002; Lhatoo et al., 2001;

Lindsten et al., 2000) or later (Hauser et al., 1980). In contrast, no such late increase in mortality was observed in the Icelandic study (Olafsson et al., 1998). This temporal mortality trend primarily appears to be significant only among people with symptomatic epilepsy although, as shown above, the SMR for people with idiopathic/cryptogenic epilepsy in the NGPSE was significantly elevated for the first time during the last ten years of follow-up (Section 4.2.4; Neligan et al., 2011a).

If such a late increase in mortality risk does occur, it raises the question as to why this should occur. The persistent increase in observed SMR noted in the NGPSE cohort occurs despite the fact that $71 \%$ of those who died were in terminal remission at time of 
death (see above), similar to that found in another study (Annegers et al., 1979). This is further strengthened by the finding that, at the most recent follow-up conducted, over $80 \%$ of people in the cohort were in 5-year terminal remission, of whom $60 \%$ were off AEDs for five or more years (Section 4.2.4; Neligan et al., 2010b). In a previous timedependent analysis of the cohort, seizure recurrence and antiepileptic drug treatment did not seem to affect mortality (Lhatoo et al., 2001).

Whether or not the risk of premature mortality is preventable ultimately depends on what is driving this higher mortality rate. If, as has been suggested by some (but not all (Camfield et al., 2002)) of the childhood epilepsy studies (Sillanpaa et al., 2010), ongoing seizures (and therefore epilepsy-related causes) are the primary cause of death, then rendering people with severe epilepsy seizure-free either by surgery (Bell et al., 2010; Sperling et al., 1999) or by medication in people with remote symptomatic epilepsy (Strauss et al., 2003) will reduce the mortality. Indeed, it has been suggested in one study that people rendered seizure free do not have an increased mortality rate (Mohanraj et al., 2006).

Patient education regarding the risk of drowning, the importance of AED compliance (AED non-compliance may be associated with increased mortality (Fraught et al., 2008)) and a discussion on the possibility of SUDEP (Morton et al., 2006) may further modify the mortality risk. If, on the other hand, the long-term mortality is primarily due to non-epilepsy related causes as suggested by our recent results and others (Lhatoo and Sander, 2005), then modifying the long-term mortality risk may not be possible until a better understanding of the patho-physiology of mortality in epilepsy is obtained. Highlighting to health-care professionals that people with a history of epilepsy are at higher risk of dying from unrelated causes such as pneumonia, may help modify this risk. 


\subsubsection{Changing mortality rates over time (Study 1)}

Mortality rates are usually age standardised and are typically expressed as the number of deaths/100,000/year. They enable us to give an indication of how death in people with epilepsy varies over time.

Population-wide studies are largely based on death certificate data (O'Callaghan et al., 2000; Pritchard et al., 2004; Massey et al., 1985; Goldacre et al., 2010; Goldacre et al., 2003; Bruce et al., 2004). It is well recognised that mortality statistics based on death certificates significantly underestimate mortality rates in people with epilepsy (Bell et al., 2004; Bruce et al., 2004), but such deficiencies should not bias interpretation of secular trends unless there is evidence that the degree of under-reporting varies over time (O'Callaghan et al., 2000).

The study comparing epilepsy mortality rates (per million patient-years) in the United States and England and Wales demonstrates a significant birth cohort effect with progressively lower mortality rates in children and young people, which is likely to be as a result of improved perinatal care (O'Callaghan et al., 2000). This is supported by evidence from population studies, which suggest that the incidence of epilepsy is decreasing in children (Sander, 1993; Kurtz et al., 1998; Sillanpaa et al., 2006). The apparent decrease in mortality rates shown in the study comparing rates in 33 countries about 20 years apart (Massey and Schoenberg, 1985) may represent an age-specific effect, with the decreased mortality rates reflecting the decreasing mortality rate in children mirrored by the falling incidence of epilepsy in children. There are also methodological differences between the two studies. In contrast there is no evidence that the mortality rates from epilepsy have decreased since the 1970s (Bruce et al., 
2004; Goldacre et al., 2010; Hauser et al., 1980; O'Callaghan et al., 2000; Pritchard et al., 2004).

Most of the population-based studies provide mortality rates in people with epilepsy up to the 1990s and so do not include many people taking the new AEDs. One study (Goldacre et al., 2010) does, however, examine mortality in England between 1996 and 2006 with no significant change in mortality rates in people with epilepsy during this time. The impact of the new AEDs in reducing mortality is, therefore, unclear and further studies are needed. Over the last decade, certifying physicians have been encouraged to mention epilepsy and, where appropriate, SUDEP on death certificates, following the finding that previously epilepsy was frequently not included even in death certificates of people who had active epilepsy (Hanna et al., 2002). If this encouragement was heeded one would expect that the number of deaths attributed to epilepsy would have increased. This does not appear to be the case (Goldacre et al., 2010), suggesting the possibility that the actual number of deaths may have fallen although no firm data are available to support this idea.

Further prospective incident cohort studies with long-term follow-up are urgently needed to confirm that people with epilepsy have a persistently elevated risk of premature death despite good seizure control, and more importantly to try to explain such a finding in order that this risk can be modified if possible.

Similarly more general population studies examining mortality rates attributable over the past twenty years are needed to determine if the large increase in the number of treatment options has impacted on mortality rates. 


\subsection{The long-term prognosis of people with epilepsy (Study 2)}

Long-term follow-up studies are important in determining the course and prognosis for people with epilepsy. The significance and inference that can be drawn from the findings from such studies are influenced by the cohort size, study population (paediatric or adult), setting (hospital- or population-based) and methodology (retrospective or prospective).

The NGPSE is the largest prospective long-term study of people with epilepsy in existence with a mean duration of follow-up of 15.9 and 16.9 years for those with definite and possible epilepsy respectively. Few other studies have examined the prognosis for people with epilepsy with a follow-up period greater than ten years and the majority of these studies have been in paediatric cohorts (Annegers et al., 1979; Okuma et al., 1981; Brorson and Wranne 1987; Oka et al., 1989; Wakamoto et al., 2000; Lindsten et al., 2001; Sillanpaa and Schmidt 2006; Geerts et al., 2010). Findings of other long-term studies are shown in Table 6 (Page 44).

Overall the reported rates of people in terminal remission in these studies range from approximately $60-80 \%$ with the lower ranges generally in studies with shorter follow-up $(<15$ years) and all were in paediatric populations. The figures from the NGPSE represent the highest proportion of people in terminal remission reported to date $(80.6 \%$ in those with definite epilepsy and $86.6 \%$ in those with possible epilepsy), in part influenced by the long period of follow-up attained in this study. In examining the two other population-based cohorts with prolonged follow-up from Rochester and Finland, it is evident that the number of people entering terminal remission continues to increase with longer follow-up. In the Rochester cohort $61 \%$ of the cohort was in TR at 10 years and $70 \%$ at 20 years (Annegers et al., 1979). In the Finnish study the proportion in TR increased with longer follow-up: 15\% in TR at five years, $48 \%$ at ten years, $52 \%$ at 15 years, $56 \%$ at 20 years, $60 \%$ at 30 years, $62 \%$ at 35 
years and $70 \%$ at 40 years follow-up. Overall $81 \%$ of this cohort had achieved a seizure free period of at least five years with or without subsequent relapse (Sillanpaa et al., 1998; Sillanpaa 2000; Sillanpaa and Schmidt, 2006). Such findings are similar to those in the NGPSE. In the previous analysis $96 \%$ of those with definite and possible seizures had achieved a one year remission, $93 \%$ a three year remission and $71 \%(67 \%$ from the $1^{\text {st }}$ seizure) by nine years follow-up (Cockerell et al., 1997). Many (82.3\%) were in TR (64\% in TR and off AEDs) after a further 15 years of follow-up (median follow-up 24.1 years). Indeed this trend of a higher proportion attaining TR appears to be continuing, as one third (21/63) of those with seizures in the last five years had experienced at least one year of seizure freedom at the end of follow-up. It is therefore probable that a proportion of these will remain seizure free with further follow-up.

Overall 164 people (44.2\%) in the cohort (96 classified as having definite epilepsy and 68 with possible epilepsy) with long-term follow-up entered early remission (five years or more seizure freedom from the index seizure) with $30(18.3 \%)$ subsequently relapsing. A further 161 people (43.4\%) (122 with definite and 39 with possible . epilepsy) became seizure free for five years or more after having had further seizures (late remission), with $13(8.1 \%)$ subsequently relapsing, giving a total of 43 people who attained five years or more seizure freedom and subsequently relapsed (Table 18, Page 130). In comparison in the Finnish paediatric cohort, 45 (31\%) entered 5-year TR within the first year with $22(48.9 \%)$ subsequently relapsing while $26(36.1 \%)$ of the 72 who attained 5-year TR late subsequently relapsed (Sillanpaa et al., 2006).

The lower rates of relapse in those with early and late remission and proportions with a relapse-remitting pattern of seizures in the NGPSE cohort $(11.6 \%$ with $48 \%$ re-entering TR) compared with the Finnish cohort (33\% with 58\% re-entering TR) may, in part be explained by the different age profile of the cohorts. In the Rochester study, which like 
the NGPSE cohort included paediatric and adult populations, approximately $75 \%$ of the cohort had attained five year of seizure freedom with 70\% in TR at the end of followup.

The higher rates of TR seen in the NGPSE compared with the other cohorts may, in part be explained by the inclusion of people with single seizures (unlike the Finnish and Rochester cohorts) who would be expected to have a lower risk of seizure recurrence than people with two or more seizures (Berg et al., 1991; Hauser et al., 1998). Similarly the inclusion of people with acute symptomatic seizures is likely to have favourably influenced the overall outcome. In a study comparing outcomes in people with a single unprovoked seizure and a first acute symptomatic seizure (stratified by aetiology), the risk of a subsequent unprovoked seizure was significantly higher at ten years follow-up in people in the first unprovoked seizure group (64.8\%; 95\% CI: 55.1, 74.4) compared with people in the acute symptomatic seizure group (18.7\%; 95\% CI: 13.7, 25.4, $\mathrm{p}<0.001$ ) (Hesdorffer et al., 2009).

For people with an initial single seizure, $37.7 \%(95 \%$ CI 95\% 29, 47) with a definite epileptic seizure had no further seizures at a median follow-up of 24 years. In a previous report from the NGPSE cohort, the recurrence rate after a seizure for all people with definite epilepsy $(n=564)$ was $78 \%(95 \%$ CI: 74,81$)$ at three years. This figure was lower, however, (recurrence rate $56 \%(95 \% \mathrm{CI}: 51,60))$ at three years when the analysis was confined to people in whom the index seizure was also the first seizure $(n=252)$ (Hart et al., 1990); these people are those designated as presenting with a single seizure in the current analysis. In most cases where the index seizure was not the first seizure, the previous seizures were focal. Consequently the figure of $37.7 \%$ of people prospectively followed after a single seizure, who remain seizure free at 24 years, indicates that only an additional six percent of people had a first relapse (recurrence rate 
$62 \%$ compared with $56 \%$ at three years) in the 21 intervening years of follow-up. This emphasises the finding that the risk of relapse following a single seizure is highest in the initial two to three years after the seizure with relatively few relapsing thereafter.

A meta-analysis of the recurrence rate after a single seizure in prospective studies found a median recurrence rate of $40 \%$, but all of these studies had a follow-up of less than five years (Berg and Shinnar, 1991). Retrospective cohort studies of people with single seizures typically report higher recurrence rates. The Rochester cohort reported a recurrence rate of $48 \%$ at three years and $56 \%$ by five years (Annegers et al., 1986). In a prospective study from Sweden of 107 people aged $\geq 17$ years with a single unprovoked seizure, the recurrence rate was $58 \%$ at ten years with nobody having a reported first recurrence after 750 days (Lindsten et al., 2001b). As with the Swedish study results from the NGPSE suggest that the risk of recurrence after a single seizure plateaus after 10 to 15 years follow-up.

In this analysis the only factors found to influence the long-term risk of seizure recurrence were aetiology and seizures before the index seizure. A higher frequency of seizures, before or after the initiation of treatment, appears to be predictive of long-term seizure outcome (MacDonald et al., 2000; Sillanpaa and Schmidt, 2009). Similarly aetiology appears to be an important determinant of long-term prognosis, a finding in keeping with other studies (Annegers et al., 1979; Brorson and Wranne, 1987; Sillanpaa and Schmidt, 2009; Geerts et al., 2010). In particular children with seizures associated with a congenital deficit had the worst prognosis even though over half were in TR at last follow-up. In contrast, people classified as having acute symptomatic seizures have the best prognosis with less than one in ten still having seizures at the end of follow-up. Interestingly this good prognosis with regard to seizure control was not seen in the mortality analysis with people with acute symptomatic seizures having a comparable 
SMR to those with remote symptomatic seizures even with the exclusion of those who died within the first year of follow-up (See Section 4.1). People classified as having idiopathic/cryptogenic epilepsy had a slightly better prognosis than people classified as having remote symptomatic epilepsy in the early years of follow-up (Cockerell et al., 1997) but this difference seems to have disappeared with longer follow-up.

One of the major limitations of the NGPSE is the aetiological classification used in people with definite epilepsy. Such a classification was based purely on the presumed temporal cause of the seizures. In particular no distinction was made between people with IGE and cryptogenic partial epilepsy. In saying that, it must be recognised that the NGPSE started several years before the ILAE published the classification of epilepsy syndromes (ILAE, 1989). Only $33.6 \%$ of people in the NGPSE cohort could be confidently classified into specific epilepsy syndromic categories when subsequently analysed (Manford et al., 1992). Consequently the latest findings from the NGPSE, while indicating an excellent prognosis for the majority of people with epilepsy and especially for people with an initial provoked seizure, can provide little information on the prognosis for people with specific epileptic syndromes.

The finding that an increasing proportion of people become seizure free with longer follow-up is encouraging. This study suggests that approximately $80 \%$ of people with epilepsy will achieve TR challenges the often cited statistic that $30-40 \%$ of people with epilepsy will ultimately have refractory epilepsy (Kwan and Brodie, 2000). Despite its limitations, the long-term follow-up data from the NGPSE cohort provides important information necessary for counselling people about the prognosis of epilepsy. 
In summary further long-term prospective general population-based studies are necessary to replicate this finding. Future studies need to be rigorous in stratifying people both by aetiology and epilepsy syndrome. This in turn may allow clinicians to give people with specific aetiological and syndromic diagnoses a more accurate longterm prognosis specific to their particular form of epilepsy. 


\subsection{The long-term prognosis of people with febrile seizures (Study 2)}

It is well recognised that children with febrile seizures are at increased risk of developing epilepsy. In prospective community based studies the reported risk is from two to seven percent depending on the duration of follow-up (and can be compared with the general population risk of $1.2 \%$ by age 24 (Hauser et al., 1993)). Children with CFSs are at higher risk than those with SFSs (Chungath and Shorvon, 2008). The main problem with the majority of such prospective cohort studies is the relatively short follow-up period (NCCP -seven years; Oakland Study - five years) therefore missing people who develop epilepsy later. In addition, short-term studies cannot provide information about the risk of developing epilepsy compared with that in the agematched population.

At 20 years after the index febrile seizure, the estimated percentage of people who had developed epilepsy was $6.7 \%(95 \%$ CI $4,11 \%)$ with three additional people having a single afebrile seizure, a rate which is comparable to that of other long-term studies (Annegers et al., 1987; Vestergaard et al., 2008). In the previous reported analysis, 12 children (6\%) had developed epilepsy after a median follow-up of 11.2 years while two had a single epileptic event (one person had two seizures within a 24 hour period) (MacDonald et al., 1999). In this follow-up, two additional people had developed epilepsy while one further person had a single epileptic seizure. Of these one who developed epilepsy and the person with a single seizure did so within the follow-up period of the last analysis (within a median follow-up of 11.2 years) but further followup information was not available at the time (MacDonald et al., 1999). Consequently only one further person with a history of febrile seizures developed epilepsy during an additional ten years of follow-up. In the last analysis the only factor associated with a 
significantly increased risk of developing subsequent epilepsy was a history of four or more febrile seizures (OR $9.4(95 \%$ CI 1.5, 5.7), $\mathrm{p}<0.015)$.

In the Danish study the risk of developing epilepsy increased with increasing number of hospital admissions for febrile seizures $(19.7 ; 95 \%$ CI 17.9, 21.7) compared with children without a history of febrile seizures (Vestergaard et al., 2008). In this study the risk of newly developing epilepsy decreased the longer the length of time since the febrile seizure. The rate of epilepsy was increased 26-fold in the first three months following a febrile seizure, decreasing to a three-fold increase after eight years but remaining significantly elevated throughout the 23 years of follow-up (Vestergaard et al., 2008).

In contrast, in the NGPSE we found that the incidence of epilepsy was no longer significantly elevated in the $15-20$ age group, which corresponds to 10 to 20 years of follow-up; numbers are small, however, with only one person with febrile seizures developing epilepsy in this age group and contributing to the SIR. Such a finding suggests that the risk of developing epilepsy after a febrile seizure may be comparable to that of the general population after 20 years.

Others have suggested three possible reasons for the association between febrile seizures and later epilepsy (Vestergaard, 2008): that febrile seizures are the first manifestation of epilepsy; that they may be an age-specific marker of susceptibility to seizures; and that prolonged febrile seizures may damage the brain with the consequent potential for the development of epilepsy. Our data and that of others suggest that most febrile seizures are not the first manifestation of epilepsy. Our view would tend towards the second and third proposed reasons - that people with febrile seizures may have an inherent susceptibility to seizures or that prolonged febrile seizures may damage the 
brain in some. We suggest that other factors also affecting seizure susceptibility may influence or indeed determine whether or not epilepsy develops. The current concept of epileptogenesis suggests that it is a progressive dynamic process of molecular and cellular changes that can occur after an acute insult (febrile seizure, head injury, acute stroke) which alters neuronal excitability with, in some people, a resultant latency period (an operational term referring to the period of time between an acute insult and the first spontaneous epileptic seizure) (Pitkanen and Lukasiuk, 2011; Pitkanen, 2010). In experimental models of epilepsy the latency period and the proportion of animals which develop epilepsy vary depending on the epileptogenic insult (Pitkanen et al., 2007). Our data suggest that febrile seizures (and other insults) may induce a timedependent process where, if spontaneous seizures do occur, they will do so within a predictable time frame as in experimental models, but that, on their own, febrile seizures are insufficient to result in epilepsy. This view of a multi-factorial epileptogenic process is supported by the finding in some studies that people with a positive family history of epilepsy are at higher risk of developing spontaneous seizures after febrile seizures (Vestergaard et al., 2008) or a head injury (Christensen et al., 2009).

In summary it is clear that this finding needs to be confirmed by further large long-term prospective incident cohort studies of children with febrile seizures, with clinical information on seizure numbers, duration and seizure type. Such large cohorts will clearly need to be stratified by ethnic groups, particularly as children from certain ethnic backgrounds (Asian) and regions (Guam) appear to be at higher risk of febrile seizures which may be genetically pre-determined. It may therefore be true that children of different ethnicities have similarly different rates of people developing epilepsy longterm. If the findings in this study are confirmed by other long-term studies, this would 
be of significant value in counselling anxious parents of the long-term risk of their child developing epilepsy in the aftermath of a febrile seizure. 


\subsection{The prognosis of chronic epilepsy (Studies $3 \& 4$ )}

\subsubsection{Seizure patterns in chronic epilepsy}

Whilst it is fully accepted that the majority of people with epilepsy who attain seizure remission do so early in the course of treatment, the related proposition that, if early treatment is unsuccessful, then the individual is likely to develop chronic epilepsy resistant to any treatment is more contentious (Kwan and Brodie, 2002). Based on this proposition, the ILAE Commission on Therapeutic Strategies has proposed that drug resistant epilepsy be defined as failure of adequate trials of two tolerated, appropriately chosen AED schedules (Kwan et al., 2010). There is, however, evidence that many people enter remission after several years of continuous seizure activity and after trials of more than two drugs (Annegers et al, 1979, Berg et al., 2006; Sillanpaa and Schmidt, 2006; Callaghan et al, 2007; Luciano and Shorvon, 2007; Del Felice et al., 2010), but how these data fit into the view of prognosis has not been established. Research on prognosis has largely focused on early predictors of subsequent intractability and identified factors have included: failure to respond to the first two appropriate AEDs tried (Kwan and Brodie, 2000), high seizure density prior to commencing treatment (MacDonald et al., 2000), epilepsy syndrome and seizure type and aetiology (Semah et al., 1998).

We identified patterns of remission and relapse during the course of their epilepsy in people with chronic epilepsy (defined here as those with active epilepsy at least five years after diagnosis). All participants had, by definition, drug resistant or refractory epilepsy at the time of recruitment and yet we found that a period of remission (seizure freedom for two years or more) had occurred in $30 \%$ (i.e. these people had the 
‘intermittent pattern' of epilepsy). The clinical and demographic features of this group were similar to those who had a continuous pattern of epilepsy, except that those with the intermittent pattern had less frequent seizures (as judged by the number in the previous year) and had taken a smaller total number of antiepileptic drugs - although this may simply reflect the shorter time period of active epilepsy.

We did not include people in early or late terminal remission in the analyses as the majority of such individuals are unlikely to be attending a tertiary epilepsy clinic. An estimate of the numbers of these people can only be gathered from population-based cohorts of people followed from the onset of their condition. Several such studies have been carried out; in one population-based study of 6,000 people, 93 people with a history of epilepsy were identified, of whom 25 (27\%) had a continuous pattern of seizures, $13(14 \%)$ an intermittent seizure pattern and $55(59 \%)$ were in remission (Goodridge and Shorvon, 1983). Similarly in a prospective cohort of 144 children with epilepsy followed for a median of 40 years, 97 (67\%) achieved terminal remission and $27(19 \%)$ had a continuous pattern of seizures (Sillanpaa and Schmidt, 2006).

In the current study neuro-imaging findings were confined to three principal pathologies (hippocampal sclerosis, cortical dysplasia and cerebro-vascular disease), which collectively accounted for $83 \%$ of abnormalities identified in people with a continuous seizure pattern and $77 \%$ in those with an intermittent seizure pattern (Table 20, Page 140). We found no difference in the proportion of people with these findings or with normal imaging between the two seizure pattern groups.

In conclusion, there seems to be considerable heterogeneity in seizure patterns in people who do not enter long-term remission in the early years after diagnosis. About one third will attain significant periods of seizure freedom, and these are people with less severe 
epilepsy (measured by seizure frequency in the year before remission and who had previously taken fewer AEDs). Entering early remission does not, however, guarantee long-term seizure freedom; in this study, each person had refractory epilepsy, yet one third of the intermittent group had had an initial remission in the first years after diagnosis. Similarly in a prospective study of early seizure pattern, $40 \%$ of people with early remission (of at least one year) had relapsed after five years with $25.3 \%$ ultimately developing drug-resistant epilepsy (Schiller, 2009). 


\subsubsection{The impact of medication change in chronic epilepsy}

In the study of medication changes, of the 139 people on whom prolonged follow-up was available, 67 (48 \%) had a greater than 50\% reduction in seizure frequency compared with $51(36.7 \%)$ at the previously published follow-up. This underlies the importance of persevering with treatment as eventually almost half will have a sustained period of significant improvement in seizure control. We found, however, that a significant proportion (approximately 50\%) of people who achieve remission will subsequently relapse on extended follow-up, which is similar to what others have found (Callaghan et al., 2007; Choi et al., 2008; Berg et al., 2009). In one of these studies, $10.7 \%$ attained at least one year seizure remission with $25 \%$ of those subsequently relapsing within 3.8 years. In an extended follow-up, $60 \%(15 / 25)(13 \%$ of total) who had one or more years seizure freedom subsequently relapsed with an $81 \%$ probability of seizure relapse after five years. No factors were found to be predictive of subsequent relapse (Choi et al., 2011). On the other hand in the extended follow-up period in the current study there were some people who became seizure free who had not been seizure free in the previous study (Luciano and Shorvon, 2007). Indeed six $(25 \%)$ of those who relapsed after becoming seizure free during the study were in remission again at the end of follow-up. In addition two people had two periods of seizure freedom (of at least one year) with a further relapse, while two people with JME had a recurrence of their myoclonus but no generalised tonic-clonic seizures. Similarly two other people, both of whom had significant periods of seizure freedom (one with a seizure-free period of six months and the other with two separate periods of one year and eighteen months of seizure freedom) but were not seizure free at either end point, had a complete cessation of generalised seizures, suggesting that even in those who subsequently relapse, their seizure control can be significantly better as a result of medication changes. 
Of the 16 people in whom no further follow-up information was available, five were seizure-free, five had a greater than 50\% improvement while the remainder (six) had no significant change in seizure frequency at the time of the last analysis (2004). It is possible that some of this group are no longer attending a tertiary referral centre because their seizures remain under complete control or significantly improved.

In the original analysis three factors (epilepsy duration, number of previous AEDs and epileptic syndrome) were found to be predictive of a better outcome. In this analysis, the only factor associated with seizure outcome at the end of follow-up was seizure outcome at the end of the earlier follow-up, suggesting that even in refractory epilepsy, the earlier that seizure remission is attained the higher the probability that seizure freedom will be maintained. The previous number of AEDs ( $<$ or $\geq 5$ AEDs) tried approached statistical significance with a lower number of concomitant AEDs statistically significant in predicting a better long-term seizure prognosis, underlying the importance of the number of previous drug failures in predicting seizure prognosis, a finding replicated in some (Callaghan et al., 2007; Schiller and Najjar, 2008) but not by others (Choi et al., 2008; Berg et al., 2009). In one of these studies, $42 \%$ of 478 people with newly treated epilepsy responded to the first AED, $17 \%$ to the next two to five AEDs, while no one became seizure free after six or more AEDs (Schiller and Najjar 2008), leading to the suggestion that absolute drug-resistance occurs after failure of five AEDs. In this study $31 \%$ (8) of those seizure-free at last follow-up had six or more previous drug failures although only three had tried more than six previous AEDs. Moreover one third of people seizure-free at last follow-up had relapsed after attaining initial seizure remission before becoming seizure free again. Similarly we identified 32 individuals in the seizure patterns study (study 3) with previously chronic epilepsy who 
had entered late terminal remission, and in this group the median number of AEDs was 6 (IQR $4.5,8)$ suggesting that there is always a chance of becoming seizure-free irrespective of the number of AEDs tried previously.

Neither epilepsy duration nor the epilepsy syndrome was predictive of long-term seizure outcome. Similarly no factors were identified to predict subsequent relapse once a person had become seizure free. (The finding of a higher proportion of people with a longer duration of epilepsy in the seizure free group is likely to be due to the small numbers).

The number of previous AED failures is central to the recently proposed definition of drug-resistant epilepsy by the ILAE (Kwan et al., 2010). It is recommended that epilepsy be classified as treatment resistant when a person continues to have seizures despite trials of at least two appropriately selected AEDs, assessed for a sufficient length of time to allow an accurate evaluation of seizure frequency. AEDs which are discontinued because of side-effects are not considered drug failures. Moreover treatment success is defined as a subsequent seizure freedom of one year or a period of seizure freedom three times the duration of the previous inter-seizure interval, whichever is longer (Kwan et al., 2010). One of the problems with this proposal is that for an individual with infrequent seizures a classification of drug resistant epilepsy may take several years to be established.

The principal rationale for identifying early predictors of potentially refractory epilepsy is so that the evaluation of alternative means of treatment (e.g. surgery) can be initiated earlier in the course of epilepsy. One possible means of predicting treatment failure is through the use of trans-cranial magnetic stimulation to measure cortical excitability before and after initiation of drug treatment in people with active epilepsy. Failure of 
normalisation of cortical excitability four to 16 weeks after starting the AED was predictive of pharmaco-resistance (Badawy et al., 2010). This is based on experimental data and is therefore speculative, with its potential application to the clinical management of people with pharmaco-resistant epilepsy yet to be established. Moreover this is likely to be available only in a small number of tertiary referral centres. This does, however, raise the interesting possibility of identifying people with treatment resistant epilepsy by means other than clinical response to sequential AED trials in the future.

Only a small proportion of people with treatment resistant epilepsy are suitable candidates for epilepsy surgery (Lhatoo et al., 2003). In this cohort less than four percent underwent epilepsy surgery despite all people with appropriate epileptic syndromes being evaluated for the possibility of epilepsy surgery. Consequently further medication changes offer the only real prospect of seizure freedom for the majority of people with treatment resistant epilepsy. This type of study demonstrates the heterogeneity of chronic epilepsy but more importantly that persistence with medication changes in the face of apparent intractability will result in nearly half having a significant reduction in seizure frequency while approximately one third will become seizure free for one or more years. Given the complexity of their epilepsy such individuals are best managed in dedicated specialist epilepsy clinics. Long-term prospective cohort studies of people with chronic epilepsy are needed to determine prognosis and the effect of treatment. Despite this, it is clear that even if a significant proportion of people with chronic epilepsy who become seizure-free will subsequently relapse, long-periods of seizure freedom or significantly improved seizure control in the absence of complete seizure control are of great value and worth striving for. 


\subsubsection{A prognostic model of epilepsy (Studies 2-4)}

The main objective of these studies is to contribute to an understanding of the temporal patterns of the prognosis of epilepsy. A fairly consistent picture is emerging. The findings from these studies that the ratio of intermittent: continuous patterns in chronic epilepsy is approximately 1:2 and that about a quarter of people with apparent refractory epilepsy will achieve late long-term remission, can be added to those of others allowing a synoptic model of prognosis to be developed (Figure 9).

Figure 9: Proposed prognostic model of epilepsy. 


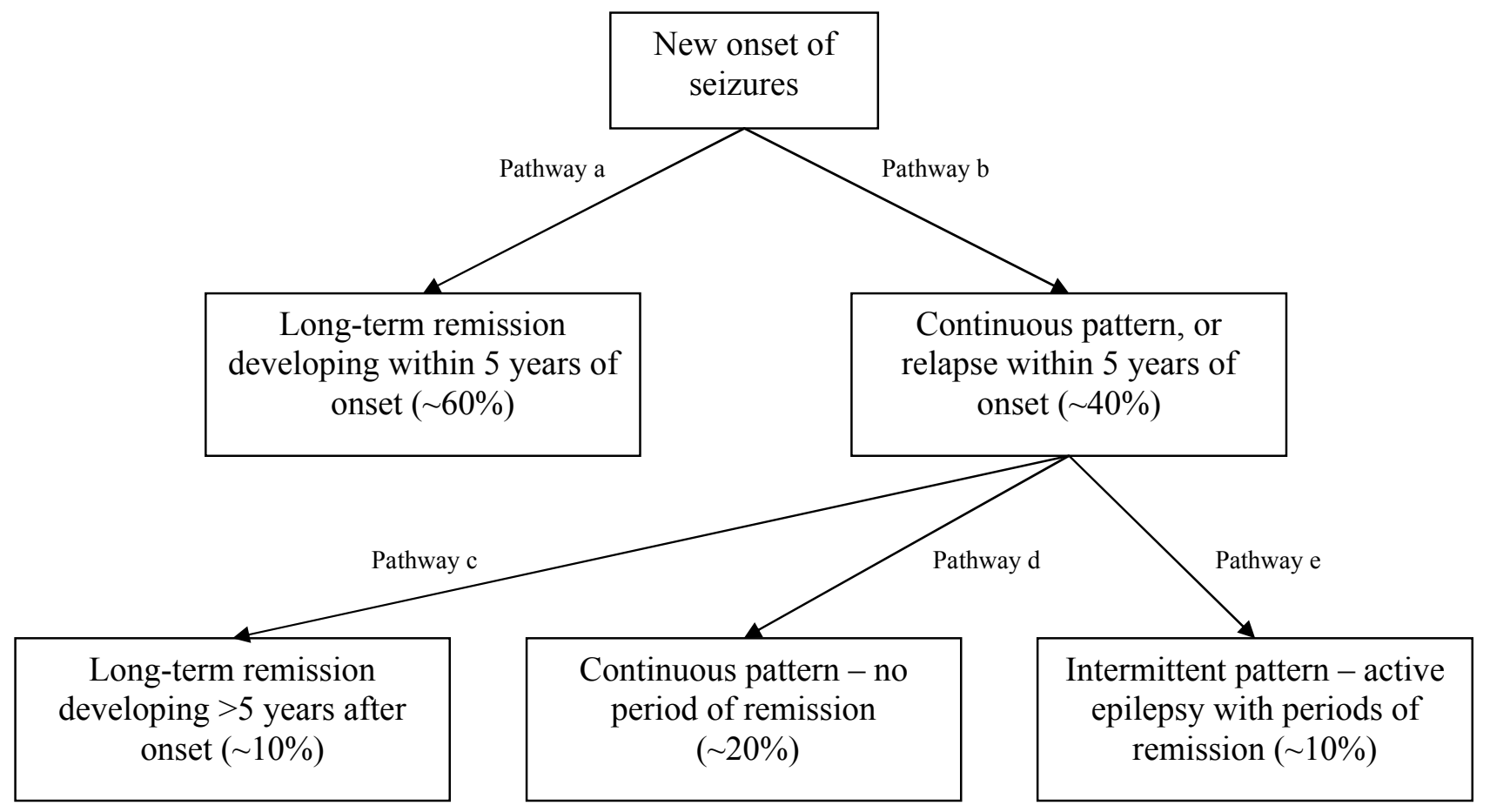


In this model, about $60 \%$ of people developing epilepsy will go into long-term (probably permanent) remission within five years of diagnosis (pathway a). This has been long suggested by epidemiological and prospective clinic based studies (Annegers et al., 1979; Goodridge and Shorvon, 1983; Sander, 1993; Cockerell et al., 1997; Kwan and Brodie, 2000; Berg et al., 2001; Lindsten et al., 2001, Kwan and Sander, 2004). To what extent this good prognosis simply represents the natural history of benign epilepsy or is a treatment effect is not known - but it is probable that both play a part. Studies from resource-poor settings suggest that at least a third of people with epilepsy will enter spontaneous remission without remission (Sander, 1993; Placencia et al., 1994; Nicoletti et al., 2009). It could therefore be postulated that treatment directly induces seizure freedom in only about half of those with early remission, although direct comparisons of different populations are not possible.

Other people (thus approximately $40 \%$ of the total) still have active epilepsy at five years after onset (i.e. are classified as having 'chronic' epilepsy; pathway b). A proportion of these people will enter subsequent long-term remission (pathway c). Exactly how large this group is not entirely clear, but interpreting data from the epidemiologically-based studies it can be suggested that this may apply to about ten percent of all people with epilepsy (Annegers et al, 1979; Sillanpaa and Schmidt, 2006). Included in this group are people with some of the age-specific epilepsy syndromes.

Other people have continuous epilepsy, with no periods of remission (pathway d), or epilepsy with an intermittent pattern (pathway e). Our study suggests that the proportion of people following these two pathways is about 2:1 (Neligan et al., 2011b). On this basis, one can estimate that about ten percent of people with epilepsy overall follow an 
intermittent pattern (pathway e) and about $20 \%$ a pattern of continuous epilepsy (pathway d).

The influence of treatment on long-term prognosis is not known, in the absence of comparative studies with untreated groups at each of these pathways. Clinical experience, however, suggests that therapy can have a significant effect in people with chronic epilepsy, as in those newly diagnosed. Of course, it needs to be emphasised that seizure freedom is not the only good outcome, and therapy will often reduce the severity, duration or frequency of seizures in beneficial ways which are not represented in this model. 


\subsection{The prognosis of status epilepticus (Study 5)}

The over-arching theme of this thesis is concerned with various aspects of the long-term prognosis of epilepsy yet in considering the prognosis of status epilepticus, outcome is primarily defined as short-term mortality (30-day case fatality rates in most studies). Indeed there is only study that examines long-term prognosis (ten-year mortality) following an episode of SE, suggesting that status epilepticus alone does not influence long-term mortality (Logroscino et al., 2002). Consequently any examination of longterm prognosis following an episode of SE is therefore better defined in terms of morbidity (for example resultant neurological or cognitive deficits or the development of resultant epilepsy) in survivors (Neligan and Shorvon, 2011).

The primary aim of carrying out a systematic review of the prognosis of SE was to examine the impact of the underlying cause on outcome. We found that the underlying aetiology is the primary determinant of prognosis of SE (Neligan and Shorvon, 2010).

SE associated with certain aetiologies such as low AED levels and alcohol abuse have a relatively good prognosis with a reported mortality in the case series of $<10 \%$, while other aetiologies such as metabolic disorders, cerebrovascular disease and particularly anoxia/hypoxia are associated with much poorer outcomes. The relative frequencies and associated mortality rates are summarised in Table 32 . 


\begin{tabular}{lll}
\hline Aetiology & Proportion of cases of SE & $\begin{array}{l}\text { Associated acute mortality } \\
\text { in people with SE }\end{array}$ \\
\hline Drug reduction/withdrawal, & $10-20 \%$ & $0-10 \%$ \\
poor compliance or low AED & & \\
levels & & \\
Cerebrovascular disease & $10-40 \%$ & $20-60 \%$ \\
Metabolic disorders & $5-15 \%$ & $10-35 \%$ \\
Acute CNS infections & $0-10 \%$ & $0-30 \%$ \\
Anoxia & $5-10 \%$ & $60-100 \%$ \\
Alcohol & $5-15 \%$ & $0-10 \%$ \\
Head trauma & $0-10 \%$ & $0-25 \%$ \\
Drug overdose/toxicity & $0-10 \%$ & $10-25 \%$ \\
Brain tumours & $0-10 \%$ & $0-20 \%$ \\
Cryptogenic/idiopathic* & $5-15 \%$ & $5-20 \%$ \\
\hline
\end{tabular}

Table 32: Approximate frequency and mortality of status epilepticus in different aetiologies in representative Western populations (Amalgamated figures from papers cited with an emphasis on representative studies and those with large sample sizes)

*The immune mediated encephalopathies with associated status epilepticus are likely to be responsible for a small number of these cases $(<1 \%$ of total) although exact figures for their prevalence do not exist.

It is also clear that when status epilepticus occurs in the setting of an acute neurological insult, the prognosis is worse than in people without an acute neurological insult, 
particularly in cases of stroke (Knake et al., 2006; Waterhouse et al., 1998), meningitis (Zoons et al., 2008) and post-anoxic SE (Thomke et al., 2005; Wijdicks et al., 1994). Of course, this may not be a direct causal relationship as the occurrence of status may itself also reflect the severity of the underlying pathology.

The relationship of factors other than aetiology (duration of SE, EEG findings, level of consciousness and age) to the prognosis of SE is less clear, in part because of the influence of the underlying aetiology on the other factors. It does however appear that the longer the seizure persists (typically more than an hour), the more likely is the seizure to be unresponsive to antiepileptic drug therapy, the higher the mortality and the worse the prognosis in survivors.

In contrast the clinical significance to the prognosis of PLEDs (PEDs) or persistent evidence of electrographic seizures post SE on EEG remains controversial. Their prognostic predictive value may, however, be more due to that of the underlying aetiology than to any independent influence. A lower level of consciousness with SE is clearly and consistently associated with a poorer prognosis, although this probably reflects the underlying aetiology, in particular anoxic damage.

The outcome and mortality of SE in children and in adults varies, with the best outcomes in young children and the worst in the elderly. The reason is partly related to the different underlying aetiologies at different ages, for example febrile status epilepticus in children and post-anoxic brain injury and degenerative brain disease in the elderly, which have different prognoses. Despite this, children and young adults appear to have consistently better outcomes (morbidity and mortality) following an episode of SE when analysed by individual aetiologies compared with older adults and 
the elderly (DeLorenzo et al., 1996; Chin et al., 2006) (Table 31, Page 188). It is likely therefore (although not definitively demonstrated in published studies) that age is an important prognostic factor in SE, independent of aetiology. 


\subsection{Problems facing epidemiological research in the UK: lessons from the NGPSE}

The experience of the NGPSE research team offers a window on the obstacles that epidemiologists currently face in the UK, in particular with regard to multi-centre research. British epidemiology has a proud pedigree with the pioneering work of Sir Richard Doll in the 1950s (Doll and Hill, 1954) among others, yet the UK of today appears to be a particularly hostile environment for prospective epidemiologists as a result of the bureaucratic quagmire of multi-level approvals by different health authorities which are necessary before any research can be undertaken.

The European Data Protection Act 1998 (UK Parliament Acts 1998) and the Health and Social Care Act 2001 (British Parliament 2001) irrevocably altered the research environment in the UK, increasing the time, cost and effort required before all the necessary approvals can be obtained (Lawlor and Stone 2001). Such a changing environment is also reflected in the dwindling number of UK patients recruited into clinical trials, decreasing from six percent of global numbers in 2000 to two percent in 2006 (Lancet, 2011).

In 2007 it seemed appropriate to send a further follow-up to GPs about survivors of the 792 people with definite or possible epilepsy and the 220 people with febrile seizures in the NGPSE. When the NGPSE was set up in 1984, full ethics approval was obtained from the local ethics committee but, as was usual at the time for purely observational studies, consent was not required from each individual recruited. Following receipt of ethics approval for the study in November 2007, application was made to the Patient 
Information Advisory Group for Section 60 exemption of the Health and Social Care Act 2001 for the study as it was felt that obtaining full informed consent from all people in the study would be profoundly detrimental to case ascertainment. This was subsequently conclusively demonstrated by our experience in Scotland where Section 60 exemption was not applicable.

PIAG is a body created by Section 60 of the Health and Social Care Act 2001 to advise the Secretary of State on cases where it may be justifiable to allow the release of patient identifiable information for research or NHS activities to proceed without patient consent (Higgins, 2003). The application for Section 60 exemption is necessarily detailed, taking several months to complete (Coleman et al., 2003). Overall it took approximately eight months from original submission to written confirmation of Section 60 exemption, a timescale comparable to, or more favourable than, the experience of others (in part influenced by the fact that the committee now meets bi-monthly as opposed to quarterly as previously) (Malfroy et al., 2004; Metcalfe et al., 2008). Approval of PIAG in granting Section 60 to the study was contingent on a significant reduction in the amount of clinical information that could be requested (see Appendices 1 and 2) in addition to the requirement for consent in certain cases. It must nevertheless be acknowledged that Section 60 exemption was critical for this stage of the study without which we would have struggled to get information from adequate numbers of individuals, to the point of rendering any conclusions drawn from the study meaningless.

Without Section 60 exemption, consent would have been required from all people included in the study; a situation we anticipated would be the case in Scotland (where the Health and Social Care Act 2001 and therefore the provision of Section 60 
exemption did not apply, as this law was enacted after Scottish devolution). We did not, however, anticipate the decision that without Section 60 exemption (or a comparable provision), we would not be allowed access to individual's GP's details. Consequently, in Scotland, all correspondence was sent to the GPs via an intermediary service with no direct contact with the GPs. We were advised that for us to have access to an individual's GP's details would be a breach of patient confidentiality. It is, however, difficult to understand how such knowledge in any way poses a threat to confidentiality. In the end this decision sounded the death knell for the study in Scotland, for without the means of personally promoting the study to GPs with the need for consent from all individuals, the response rate was inevitably extremely poor (26\%).

One of the reasons given by PIAG for not granting Section 60 exemption following the original submission was the contention that a valid alternative to Section 60 exemption was to ask GPs to obtain consent, as stated in their original decision letter. “...the Group noted that you are intending to contact patient's GPs and this is an ideal opportunity to ask them to publicise the study and to attempt to obtain consent" Without Section 60 exemption, we would not have been allowed access to individual GP's details in England and Wales (as was the case in Scotland) which raises the question of how we could ask the GPs to obtain consent or indeed promote the study to the relevant GPs.

The PIAG's original contention that GPs can reasonably be expected to obtain consent from individuals for their continued participation in a study in existence for over twenty years is certainly debatable. Moreover the blanket requirement of consent for all people in the study raises the risk of selection bias with people with ongoing seizures more likely to consent to inclusion in a study that is directly relevant to them (as opposed to 
people in long-term remission who may even be offended by their inclusion in such a study) (Al-Shahi et al., 2005).

The study was adopted onto the NIHR CRN and the PCRN in England (which almost certainly would not have happened without Section 60 approval). As a result the study was eligible for Service Support Costs. It was agreed that GP practices would be paid if the questionnaire was returned completed and with consent where required. In certain cases, when we contacted GP practices by telephone after failure to return the questionnaire (or returning it incomplete), we discovered that many had simply forwarded the information onto the individual patient without explanation or support for the study where consent was required to complete the study (and in many cases where consent was not required). Failure of patients to return questionnaires/consent forms or reply to invitations to participate in studies is assumed by regulatory authorities to be the same as refusal to give consent yet surveys of the UK public on the use of patient identifiable information does not support this interpretation. Only 2-3\% of people typically refuse outright involvement (Barrett et al., 2006; Jackson et al., 2008) with up to $20 \%$ wishing to be informed prior to the use of their information in national databases or registries (Campbell et al., 2007). In the NGPSE we are aware of only four people who explicitly refused consent although the true figure is almost certainly higher. Nevertheless this is significantly less than the $50-60 \%$ who typically fail to respond to invitations to participate in research (Tu et al., 2004; Al-Shahi et al 2005) with higher rates achieved when the person requesting consent is directly involved in the research rather than an intermediary (Tu et al., 2004). The exclusive reliance on GPs to obtain consent in longitudinal studies is problematic with many GPs not wishing to broach the sometimes problematic issue of consent for research even when there is a financial incentive to do so. In addition, a proportion of people invited to participate in 
research will not respond because of an incomplete explanation of the study. Of note, every person who contacted the NGPSE research team for further information on the study gave consent.

Following receipt of ethical approval of the final necessary amendment to the study, application was made to all PCT R\&D departments where it was assumed that people in the study currently resided. Application was also made to the Information Centre (IC) NHS (formerly the ONS). Despite the fact that the study had been granted Section 60 exemption more than five months previously, this decision was not communicated to the IC NHS. Consequently the study had to be reviewed by the ethics committee of IC, resulting in a minor change to the patient consent form (see appendix). Similarly final release of GP details from the Data Protection Managers was subject to review by the local Caldicott Guardian in several instances. For multi-centre epidemiological project like the NGPSE, approval is required from all PCT R\&D departments, a very timeconsuming process varying from several weeks to many months depending on the PCT. This involves a process of checks and validations duplicated by other Trusts as well as ethical committees (Ethics, IC, PIAG). In some instances it appeared that the primary motivation of the R\&D department was to protect the Trust from future possible legal action rather than evaluating the validity and clinical need for the research project. In one instance an $R \& D$ department refused approval for the study, a decision that was only reversed on appeal when it was demonstrated that patient consent was unlikely to be required from the people in the study in that PCT.

In total it took almost two years from the time of submission of the ethics application to when the first questionnaires were sent out, a delay largely accounted for by the time needed to obtain all the required approvals to proceed with the study. If this was the 
norm for other epidemiological multi-centre studies, it would have disastrous consequences for case ascertainment not to mention the probability of obtaining research funding for such projects which would be seen as prohibitively expensive due to administrative delays. In the end while a further follow-up of the cohort has been completed, permission to allow this to happen was contingent on the permanent closure of the study upon completion of this follow-up and the destruction of the study records (both paper and electronic). While such a decision safe-guards individual patient data, we would strongly contend that it is clearly not in the best interests for people with epilepsy in general.

Fortunately change appears to be at hand with the recognition of the need to reduce the duplication and bureaucracy in the regulation of clinical research in the UK with the recent publication of a white paper (Liberating the NHS: report of the arm's-length bodies review) by the Academy of Medical Sciences. This paper proposes the creation of a new Health Research Agency which will incorporate the current National Research Ethics Service as well as other specialist research ethic approvals (PIAG?). In so doing, it is proposed that this will reduce the need for local checks by the PCTs other than local feasibility checks (Rawlins, 2011). It is hoped that such changes will reduce the bureaucracy and administrative delays that plagues multi-centre clinical research, thus safeguarding it for future generations. 


\section{Chapter 9: Conclusions and future directions}

The principal aims of this thesis were to study different aspects of the prognosis of people with epilepsy, in particular the long-term prognosis with regard to the risk of premature mortality, seizure prognosis and changing seizure patterns over time and the response to medication in people with established refractory epilepsy. In addition the prognosis (both short and long-term) of status epilepticus, the "maximal expression of epilepsy", is reviewed with emphasis on determining which factors determine prognosis.

Based on the data presented in this thesis there are several issues with regard to the prognosis of epilepsy that need to be addressed.

1) Further population-based studies are needed to examine the temporal changes in the number of deaths attributable to epilepsy, in particular since 1990, to gauge the impact on mortality rates of the increasing number of treatment options

2) Prospective incident studies such as the NGPSE suggest that the long-term risk of premature mortality remains significantly elevated despite the fact that the majority of people achieve terminal remission. Further prospective incident cohort studies are urgently needed to verify such a finding and to provide clues as to why people with a history of epilepsy have a persistently higher risk of premature mortality despite good control. More importantly, better understanding of the mechanisms of mortality will help determine if this higher risk can be modified. 
3) There is a paucity of long-term follow-up data in particular in adults with new onset epilepsy. It is often stated that one third of people with epilepsy will have treatment resistant epilepsy, often as justification for the need for further AEDs or treatment modalities, yet the latest data from the NGPSE suggest that such estimates may be overly pessimistic. Moreover it is possible that a third of those with supposedly refractory epilepsy will in fact have significant periods of seizure freedom, probably as a result of medication changes. Further long-term prospective incident cohort studies with both paediatric and adult populations examining prognosis in people with new onset epilepsy are needed. Such studies should be carried out with particular reference paid to aetiology and epileptic syndrome, changing seizure patterns and prognosis in refractory epilepsy in response to further AED changes.

4) Such questions may best be addressed by the creation of a national database (with patient consent) of all people with newly diagnosed or suspected epileptic seizures. The logistics and feasibility of such a project would need to be worked out but would clearly be in the best interests of all people with epilepsy, for whom much uncertainty regarding prognosis remains. 


\section{Reference List}

Abib CR, Mendoza-Sassi RA, Bech-Nappi J et al (2007) Prevalence of seizures and associated factors in children under five living in a deprived municipality of southern Brazil. Arq Neuropsiquiatr 65: 581-586.

Adachi N, Kanemoto K, Muramatsu R et al (2005). Intellectual prognosis of status epilepticus in adult epilepsy patients: analysis with Wechsler Adult Intelligence Scalerevised. Epilepsia 46: 1502-1509.

Adelöw C, Ahlbom A, Feychting M et al (2006). Epilepsy as a risk factor for cancer. J Neurol Neurosurg Psychiatry 77: 784-786.

Adelow C, Andell E, Amark P et al (2009). Newly diagnosed single unprovoked seizures and epilepsy in Stockholm, Sweden: First report from the Stockholm Incidence Registry of Epilepsy (SIRE). Epilepsia 50: 1094-1101.

Afsar N, Kaya D, Aktan S et al (2003). Stroke and status epilepticus: stroke type, type of status epilepticus, and prognosis. Seizure 12: 23-7.

Aicardi J and Chevrie JJ (1970). Convulsive status epilepticus in infants and children. A study of 239 cases. Epilepsia 11: 187-197.

Airaksinen EM, Matilainen R, Mononen T et al (2000). A population-based study on epilepsy in mentally retarded children. Epilepsia 41: 1214-1220.

Alldredge BK, Gelb AM, Isaacs SM et al (2001). A comparison of lorazepam, diazepam, and placebo for the treatment of out-of-hospital status epilepticus. N Engl J Med 345: 631-637. 
Alldredge BK and Lowenstein DH (1993). Status epilepticus related to alcohol abuse. Epilepsia 34: 1033-1037.

Almu S, Tadesse Z, Cooper P et al (2006). The prevalence of epilepsy in the Zay Society, Ethiopia--an area of high prevalence. Seizure 15: 211-213.

Al-Shahi R, Vousden C and Warlow C (2005). Bias from requiring explicit consent from all participants in observational research: prospective, population based study. BMJ 331:942.

Alstrom CH (1942). Mortality in mental hospitals with special regard to tuberculosis. Appelsbergs Boktryckeriaktieborg 297-304.

Amare A, Zenebe G, Hammack J et al (2008). Status epilepticus: clinical presentation, cause, outcome, and predictors of death in 119 Ethiopian patients. Epilepsia 49: 600607.

Amatniek JC, Hauser WA, DelCastillo-Castaneda C et al (2006).Incidence and predictors of seizures in patients with Alzheimer's disease. Epilepsia 47: 867-872.

Andersen GL, Irgens LM, Haagaas I et al (2008). Cerebral palsy in Norway: prevalence, subtypes and severity. Eur J Paediatr Neurol 12: 4-13.

Andersohn F, Schade R, Willich SN et al (2010). Use of antiepileptic drugs in epilepsy and the risk of self-harm or suicidal behavior. Neurology 75: 335-340.

Annegers JF, Dubinsky S, Coan SP et al (1999). The incidence of epilepsy and unprovoked seizures in multiethnic, urban health maintenance organizations. Epilepsia 40: 502-506. 
Annegers JF, Grabow JD, Groover RV et al (1980). Seizures after head trauma: a population study. Neurology 30: 683-689.

Annegers JF, Hauser WA and Elveback LR (1979a). Remission of seizures and relapse in patients with epilepsy. Epilepsia 20: 729-737.

Annegers JF, Hauser WA, Elveback LR et al (1979b). The risk of epilepsy following febrile convulsions. Neurology 29: 297-303.

Annegers JF, Hauser WA and Shirts SB. (1984) Heart disease mortality and morbidity in patients with epilepsy. Epilepsia 25:699-704.

Annegers JF, Hauser WA, Shirts SB et al (1987).Factors prognostic of unprovoked seizures after febrile convulsions. N Engl J Med 316: 493-498.

Annegers JF, Hauser WA, Beghi E et al (1988). The risk of unprovoked seizures after encephalitis and meningitis. Neurology 38: 1407-1410.

Annegers JF, Hauser WA, Coan SP et al (1998). A population-based study of seizures after traumatic brain injuries. N Engl J Med 338: 20-24.

Annegers JF, Hauser WA, Lee JR et al (1995). Incidence of acute symptomatic seizures in Rochester, Minnesota, 1935-1984. Epilepsia 36: 327-333.

Annegers JF, Shirts SB, Hauser WA et al (1986). Risk of Recurrence After an Initial Unprovoked Seizure. Epilepsia 27: 43-50.

Anonymous (2011). Untangling health research governance in the UK. Lancet 377: 179. Asadi-Pooya AA and Poordast A (2005). Etiologies and outcomes of status epilepticus in children. Epilepsy Behav 7: 502-505. 
Aziz H, Guvener A, Akhtar SW et al (1997). Comparative epidemiology of epilepsy in Pakistan and Turkey: population-based studies using identical protocols. Epilepsia 38: 716-722.

Badawy RA, Macdonell RA, Berkovic SF et al (2010). Predicting seizure control: cortical excitability and antiepileptic medication. Ann Neurol 67: 64-73.

Barnard C and Wirrell E (1999). Does status epilepticus in children cause developmental deterioration and exacerbation of epilepsy? J Child Neurol 14: 787-794.

Barrett G, Cassell JA, Peacock JL et al (2006). National survey of British public's views on use of identifiable medical data by the National Cancer Registry. BMJ 332: 10681072.

Bateman BT, Claassen J, Willey JZ et al (2007). Convulsive status epilepticus after ischemic stroke and intracerebral hemorrhage: frequency, predictors, and impact on outcome in a large administrative dataset. Neurocrit Care 7: 187-193.

Bauer S, Cepok S, Todorova-Rudolph A et al (2009). Etiology and site of temporal lobe epilepsy influence postictal cytokine release. Epilepsy Res 86: 82-8.

Baumann A, Audibert G, McDonnell J et al (2007). Neurogenic pulmonary edema. Acta Anaesthesiol Scand 51: 447-455.

Beghi E, Carpio A, Forsgren L et al (2010). Recommendation for a definition of acute symptomatic seizure. Epilepsia. 51: 671-675.

Beilmann A, Napa A, Hämarik M et al (1999).Incidence of childhood epilepsy in Estonia. Brain Dev 21: 166-174. 
Bell GS, Gaitatzis A, Bell CL et al (2008). Drowning in people with epilepsy: how great is the risk? Neurology 71: 578-582.

Bell GS, Gaitatzis A, Bell CL et al (2009a). Suicide in people with epilepsy: how great is the risk? Epilepsia 50: 1933-1942.

Bell GS, Gaitatzis A, Johnson AL et al (2004). Predictive value of death certification in the case ascertainment of epilepsy. J Neurol Neurosurg Psychiatry 75: 1756-1758.

Bell GS, Mula M and Sander JW (2009b). Suicidality in People Taking Antiepileptic Drugs: What is the Evidence? CNS Drugs 23: 281-292.

Bell GS, Sinha S, Tisi J et al. (2010). Premature mortality in refractory partial epilepsy: does surgical treatment make a difference? J Neurol Neurosurg Psychiatry 81: 716-718.

Benn EK, Hauser WA, Shih T et al (2008). Estimating the incidence of first unprovoked seizure and newly diagnosed epilepsy in the low-income urban community of Northern Manhattan, New York City. Epilepsia 49: 1431-1439.

Beran RG, Michelazzi J, Hall L et al (1985). False-negative response rate in epidemiologic studies to define prevalence ratios of epilepsy. Neuroepidemiology 4: 8285.

Berg AT and Shinnar S (1991). The risk of seizure recurrence following a first unprovoked seizure: a quantitative review. Neurology 41: 965-972.

Berg AT and Shinnar S (1994). The contributions of epidemiology to the understanding of childhood seizures and epilepsy. J Child Neurol 9 Suppl 2: 19-26.

Berg AT, Levy SR, Novotny EJ et al (1996).Predictors of intractable epilepsy in childhood: a case-control study. Epilepsia 37: 24-30. 
Berg AT, Shinnar S, Darefsky AS et al (1997). Predictors of recurrent febrile seizures. A prospective cohort study. Arch Pediatr Adolesc Med 151: 371-378.

Berg AT, Shinnar S, Levy SR et al (1999). Newly diagnosed epilepsy in children: presentation at diagnosis. Epilepsia 40: 445-452.

Berg AT, Shinnar S, Levy SR et al (2001). Two-year remission and subsequent relapse in children with newly diagnosed epilepsy. Epilepsia 42: 1553-1562.

Berg AT, Lin J, Ebrahimi N et al (2004). Modeling remission and relapse in pediatric epilepsy: application of a Markov process. Epilepsy Res. 60:31-40.

Berg AT and Kelly MM. (2006a) Defining intractability: comparisons among published definitions. Epilepsia: 431-436.

Berg AT, Vickrey BG, Testa FM et al (2006b) How long does it take for epilepsy to become intractable? A prospective investigation. Ann Neurol 60: 73-79.

Berg AT, Levy SR, Testa FM et al (2009). Remission of epilepsy after two drug failures in children: a prospective study. Ann Neurol; 65: 510-519.

Berg AT, Berkovic SF, Brodie MJ et al (2010). Revised terminology and concepts for organization of seizures and epilepsies: report of the ILAE Commission on Classification and Terminology, 2005-2009. Epilepsia; 51: 676-685.

Bladin CF, Alexandrov AV, Bellavance A et al (2000).Seizures after stroke: a prospective multicenter study. Arch Neurol 57: 1617-1622.

Bleck TP (1991). Convulsive disorders: status epilepticus. Clin Neuropharmacol 14: 191-198. 
Bonnett LJ, Shukralla A, Tudur-Smith C et al (2011). Seizure recurrence after antiepileptic drug withdrawal and the implications for driving: further results from the MRC Antiepileptic Drug Withdrawal Study and a systematic review. J Neurol Neurosurg Psychiatry doi:10.1136/jnnp.2010.222885

British Parliament. The Health and Social Care Bill. http://parliament.the-stationeryoffice.co.uk/pa/cm200001/cmbills/009/200/009.htm

Brodtkorb E and Sjaastad O (2008). Epilepsy prevalence by individual interview in a Norwegian community. Seizure 17: 646-650.

Brorson LO and Wranne L (1987). Long-term prognosis in childhood epilepsy: survival and seizure prognosis. Epilepsia 28: 324-330.

Bruce M, Griffiths C, Brock A et al (2004). Trends in mortality and hospital admissions asscoiated with epilepsy in England and Wales during the 1990s. Health Statistics Quaterly 21: 23-29.

Burn J, Dennis M, Bamford J et al (1997).Epileptic seizures after a first stroke: the Oxfordshire Community Stroke Project. BMJ 315: 1582-1587.

Burneo JG, Tellez-Zenteno J and Wiebe S (2005). Understanding the burden of epilepsy in Latin America: a systematic review of its prevalence and incidence. Epilepsy Res 66: $63-74$.

Callaghan BC, Anand K, Hesdorffer D et al (2007). Likelihood of seizure remission in an adult population with refractory epilepsy. Ann Neurol 62: 382-389.

Campbell B, Thomson H, Slater J et al (2007). Extracting information from hospital records: what patients think about consent. Qual Saf Health Care 16: 404-408. 
Camfield CS, Camfield PR and Veugelers PJ (2002). Death in children with epilepsy: a population-based study. Lancet 359: 1891-1895.

Carpio A, Bharucha NE, Jallon P et al (2005). Mortality of epilepsy in developing countries. Epilepsia 46 Suppl 11: 28-32.

Cascino GD, Hesdorffer D, Logroscino G et al (1998). Morbidity of nonfebrile status epilepticus in Rochester, Minnesota, 1965-1984. Epilepsia 39: 829-832.

Cavaliere R, Farace E and Schiff D (2006). Clinical implications of status epilepticus in patients with neoplasms. Arch Neurol 63: 1746-1749.

Chen JW and Wasterlain CG (2006). Status epilepticus: pathophysiology and management in adults. Lancet Neurol 5: 246-256.

Chen L, Zhou B, Li JM et al (2009). Clinical features of convulsive status epilepticus: a study of 220 cases in western China. Eur J Neurol 16: 444-449.

Chen YJ, Fang PC and Chow JC (2006). Clinical characteristics and prognostic factors of postencephalitic epilepsy in children. J Child Neurol 21: 1047-1051.

Chin RF, Neville BG, Peckham C et al (2006).Incidence, cause, and short-term outcome of convulsive status epilepticus in childhood: prospective population-based study. Lancet 368: 222-229.

Choi H, Heiman GA, Clary HM et al (2011). Seizure remission in adults with longstanding intractable epilepsy: An extended follow-up. Epilepsy Res 93:115-119

Choi H, Heiman G, Pandis D et al (2008). Seizure remission and relapse in adults with intractable epilepsy: a cohort study. Epilepsia 49: 1440-1445. 
Christensen J, Pedersen MG, Pedersen CB et al (2009). Long-term risk of epilepsy after traumatic brain injury in children and young adults: a population-based cohort study. Lancet 373: 1105-1110.

Christensen J, Vestergaard M, Mortensen PB et al (2007a). Epilepsy and risk of suicide: a population-based case-control study. Lancet Neurol 6: 693-698.

Christensen J, Vestergaard M, Pedersen MG et al (2007b). Incidence and prevalence of epilepsy in Denmark. Epilepsy Res 76: 60-65.

Chungath M and Shorvon S (2008). The mortality and morbidity of febrile seizures. Nat Clin Pract Neurol 4: 610-621.

Claassen J, Hirsch LJ, Emerson RG et al (2002). Treatment of refractory status epilepticus with pentobarbital, propofol, or midazolam: a systematic review. Epilepsia 43: 146-153.

Claassen J, Peery S, Kreiter KT et al (2003).Predictors and clinical impact of epilepsy after subarachnoid hemorrhage. Neurology 60: 208-214.

Clark LP and Prout TP (1903/4). Status epilepticus: a clinical and pathological study in epilepsy. Amer J Insanity 60 \& 61:291-306, 645-670, 81-108.

Cockerell OC, Eckle I, Goodridge DM et al (1995). Epilepsy in a population of 6000 reexamined: secular trends in first attendance rates, prevalence, and prognosis. J Neurol Neurosurg Psychiatry 58: 570-576.

Cockerell OC, Johnson AL, Sander JW et al (1994). Mortality from epilepsy: results from a prospective population-based study. Lancet 344: 918-921. 
Cockerell OC, Johnson AL, Sander JW et al (1997). Remission of epilepsy: results from the National General Practice Study of Epilepsy. Lancet 346: 140-144.

Cockerell OC, Walker MC, Sander JW et al (1994). Complex partial status epilepticus: a recurrent problem. J Neurol Neurosurg Psychiatry 57: 835-837.

Coeytaux A, Jallon P, Galobardes B et al (2000). Incidence of status epilepticus in French-speaking Switzerland: (EPISTAR). Neurology 55: 693-697.

Coleman MP, Evans BG and Barrett G (2003). Confidentiality and the public interest in medical research--will we ever get it right? Clin Med 3: 219-228.

Collaborative Group for the Study of Epilepsy (1992). Prognosis of Epilepsy in Newly Referred Patients: A Multicenter Prospective Study of the Effects of Monotherapy on the Long-Term Course of Epilepsy. Epilepsia 33: 45-51.

Commission on Classification and Terminology of the International League Against Epilepsy (1981). Proposal for revised clinical and electroencephalographic classification of epileptic seizures. Epilepsia 22: 489-501.

Commission on Classification and Terminology of the International League Against Epilepsy (1989). Proposal for revised classification and epilepsies and epileptic syndromes. Epilepsia 30: 389-399.

Commission on Epidemiology and Prognosis ILAE (1993). Guidelines for Epidemiologic Studies on Epilepsy. Epilepsia 34: 592-596.

Commission on Epidemiology and Prognosis ILAE (1997). The epidemiology of the epilepsies: future directions. Epilepsia 38: 614-618. 
Commission on Tropical Diseases of the International League Against Epilepsy (1994). Relationship between epilepsy and tropical diseases. Epilepsia 35: 89-93.

Dalmau J, Lancaster E, Martinez-Herandez E et al (2011). Clinical experience and laboratory investigations in patients with anti-NMDAR encephalitis. Lancet Neurol 10: 63-74.

Dalmau J, Gleichman AJ, Hughes EG et al (2008). Anti-NMDA-receptor encephalitis: case series and analysis of the effects of antibodies. Lancet Neurol 7: 1091-1098.

De La Court, Breteler MM, Meinardi H et al (1996). Prevalence of epilepsy in the elderly: the Rotterdam Study. Epilepsia 37: 141-147.

Delanty N, French JA, Labar DR et al (2001) Status epilepticus arising de novo in hospitalized patients: an analysis of 41 patients. Seizure 10: 116-119.

Del Felice A, Beghi E, Boero G et al (2010). Early versus late remission in a cohort of patients with newly diagnosed epilepsy. Epilepsia; 51: 37-42.

DeLorenzo RJ, Garnett LK, Towne AR et al (1999). Comparison of status epilepticus with prolonged seizure episodes lasting from 10 to 29 minutes. Epilepsia; 40: 164-169.

DeLorenzo RJ, Hauser WA, Towne AR et al (1996).A prospective, population-based epidemiologic study of status epilepticus in Richmond, Virginia. Neurology 46: 10291035.

Dent W, Helbok R, Matuja WB et al (2005). Prevalence of active epilepsy in a rural area in South Tanzania: a door-to-door survey. Epilepsia 46: 1963-1969.

Department of Health, Liberating the NHS: report of the arm's-length bodies review Available at: 
http://www.dh.gov.uk/en/Publicationsandstatistics/Publications/PublicationsPolicyAnd Guidance/DH_117691 Accessed the 30 $0^{\text {th }}$ January 2011.

De Vogli R, Mistry R, Gnesotto R et al (2005). Has the relation between income inequality and life expectancy disappeared? Evidence from Italy and top industrialised countries. J Epidemiol Community Health 59: 158-162.

Ding D, Wang W, Wu J et al (2006). Premature mortality in people with epilepsy in rural China: a prospective study. Lancet Neurol 5: 823-827.

Dodrill CB and Wilensky AJ (1990). Intellectual impairment as an outcome of status epilepticus. Neurology 40: 23-27.

Dogui M, Jallon P, Tamallah JB et al (2003).Episousse: incidence of newly presenting seizures in children in the Region of Sousse, Tunisia. Epilepsia 44: 1441-1444.

Doll R and Hill AB (1954). The mortality of doctors in relation to their smoking habits; a preliminary report. Br Med J 1:1451-1455.

Drislane FW, Blum AS, Lopez MR et al (2009). Duration of refractory status epilepticus and outcome: loss of prognostic utility after several hours. Epilepsia 50: $1566-1571$.

Dunn DW (1988). Status epilepticus in children: etiology, clinical features, and outcome. J Child Neurol 3: 167-173.

Edwards T, Scott AG, Munyoki G et al (2008). Active convulsive epilepsy in a rural district of Kenya: a study of prevalence and possible risk factors. Lancet Neurol 7: 5056. 
Ellenberg JH, Hirtz DG and Nelson KB (1986). Do seizures in children cause intellectual deterioration? N Engl J Med 314: 1085-1088.

Ellenberg JH.and Nelson KB (1978). Febrile seizures and later intellectual performance. Arch Neurol 35: 17-21.

Elwes RD, Marshall J, Beattie A et al (1991). Epilepsy and employment. A community based survey in an area of high unemployment. J Neurol Neurosurg Psychiatry 54: 200203.

Eriksson KJ and Koivikko MJ (1997). Status epilepticus in children: aetiology, treatment, and outcome. Dev Med Child Neurol 39: 652-658.

Faught E, Duh MS, Weiner JR et al (2008). Nonadherence to antiepileptic drugs and increased mortality: findings from the RANSOM Study. Neurology 71: 1572-1578.

Feksi AT, Kaamugisha J, Sander JW et al (1991). Comprehensive primary health care antiepileptic drug treatment programme in rural and semi-urban Kenya. Lancet.; 337: 406-409.

Ficker DM, So EL, Shen WK et al (1998). Population-based study of the incidence of sudden unexplained death in epilepsy. Neurology 51: 1270-1274.

Forsgren L (1990).Prospective incidence study and clinical characterization of seizures in newly referred adults. Epilepsia 31: 292-301.

Forsgren L (1992). Prevalence of epilepsy in adults in northern Sweden. Epilepsia 33: 450-458.

Forsgren L, Beghi E, Oun A et al (2005a). The epidemiology of epilepsy in Europe - a systematic review. Eur J Neurol 12: 245-253. 
Forsgren L, Bucht G, Eriksson S et al (1996a). Incidence and clinical characterization of unprovoked seizures in adults: a prospective population-based study. Epilepsia 37: 224229.

Forsgren L, Edvinsson SO, Nystrom L et al (1996b). Influence of epilepsy on mortality in mental retardation: an epidemiologic study. Epilepsia 37: 956-963.

Forsgren L, Hauser WA, Olafsson E et al (2005b). Mortality of epilepsy in developed countries: a review. Epilepsia 46 Suppl 11: 18-27.

Gaitatzis A, Carroll K, Majeed A et al (2004b). The epidemiology of the comorbidity of epilepsy in the general population. Epilepsia 45: 1613-1622.

Gaitatzis A, Johnson AL, Chadwick DW et al (2004a). Life expectancy in people with newly diagnosed epilepsy. Brain 127: 2427-2432.

Gallitto G, Serra S, La SP et al (2005). Prevalence and characteristics of epilepsy in the Aeolian islands. Epilepsia 46: 1828-1835.

Garzon E, Fernandes RM and Sakamoto AC (2001). Serial EEG during human status epilepticus: evidence for PLED as an ictal pattern. Neurology 57: 1175-1183.

Garzon E, Fernandes RM and Sakamoto AC (2003). Analysis of clinical characteristics and risk factors for mortality in human status epilepticus. Seizure 12: 337-345.

Gastaut H, Roger J, Lob,H (1967). Les États de Mal Épileptiques. Masson, Paris.

Geerts A, Arts WF, Stroink H et al (2010). Course and outcome of childhood epilepsy: a 15-year follow-up of the Dutch Study of Epilepsy in Childhood. Epilepsia 51: 11891197. 
Glaser CA, Gilliam S, Honarmand S et al (2008). Refractory status epilepticus in suspect encephalitis. Neurocrit Care 9: 74-82.

Goldacre MJ, Duncan ME, Cook-Mozaffari P et al (2003). Trends in mortality rates comparing underlying-cause and multiple-cause coding in an English population 19791998. J Public Health Med; 25: 249-253.

Goldacre MJ, Duncan M, Griffith M et al (2010). Trends in death certification for multiple sclerosis, motor neuron disease, Parkinson's disease and epilepsy in English populations 1979-2006. J Neurol 257; 706-715.

Goldberg ID and Kurland LT (1962). Mortality in 33 countries from diseases of the nervous system. World Neurol 3: 444-465.

Gomes Md MM, Zeitoune RG, Kropf LA et al (2002). A house-to-house survey of epileptic seizures in an urban community of Rio de Janeiro, Brazil. Arq Neuropsiquiatr 60: 708-711.

Goulden KJ, Shinnar S, Koller H et al (1991). Epilepsy in children with mental retardation: a cohort study. Epilepsia 32: 690-697.

Gowers,W.R.G., 1881. Epilepsy and other chronic diseases, 1st Ed. Churchill, London.

Gracia FJ, Bayard V, Triana E et al (1988). [Prevalence of neurologic diseases in Belisario Porras municipality, District of San Miguelito, Panama, 1986]. Rev Med Panama 13: 40-45.

Gracia F, de Lao SL, Castillo L et al (1990). Epidemiology of epilepsy in Guaymi Indians from Bocas del Toro Province, Republic of Panama. Epilepsia 31: 718-723. 
Granerod J, Ambrose HE, Davies NWS et al (2010). Causes of encephalitis and differences in their clinical presentations in England: a multicentre, population-based prospective study. Lancet Infect Dis 10:835-844

Gulati S, Kalra V and Sridhar MR (2005). Status epilepticus in Indian children in a tertiary care center. Indian J Pediatr 72: 105-108.

Hallstrom B, Norrving B and Lindgren A (2002). Stroke in Lund-Orup, Sweden: improved long-term survival among elderly stroke patients. Stroke 33: 1624-1629.

Hanna NJ, Black M, Sander JW et al. (2002). The national sentinel clinical audit of epilepsy-related death: epilepsy - death in the shadows. The Stationery Office, London.

Harris EC and Barraclough B (1997). Suicide as an outcome for mental disorders. A meta-analysis. Br J Psychiatry 170: 205-228.

Hart YM, Sander JW and Shorvon SD (1989) National General Practice Study of Epilepsy and Epileptic Seizures: objectives and study methodology of the largest reported prospective cohort study of epilepsy. National General Practice of Epilepsy and Epileptic Seizures (NGPSE). Neuroepidemiology 8:221-227.

Hart YM, Sander JW, Johnson AL et al (1990). National General Practice Study of Epilepsy: recurrence after a first seizure. Lancet 336: 1271-1274.

Harvey AS, Nolan T and Carlin JB (1993). Community-based study of mortality in children with epilepsy. Epilepsia 34: 597-603.

Hauser WA (1990). Status epilepticus: epidemiologic considerations. Neurology 40 Suppl 2: 9-13. 
Hauser WA (1994). The prevalence and incidence of convulsive disorders in children. Epilepsia 35 Suppl 2: 1-6.

Hauser WA, Annegers JF and Elveback LR (1980). Mortality in patients with epilepsy. Epilepsia 21: 399-412.

Hauser WA, Annegers JF and Kurland LT (1991). Prevalence of epilepsy in Rochester, Minnesota: 1940-1980. Epilepsia 32: 429-445.

Hauser WA, Annegers JF and Kurland LT (1993). Incidence of epilepsy and unprovoked seizures in Rochester, Minnesota: 1935-1984. Epilepsia 34: 453-468.

Hauser WA and Beghi E (2008). First seizure definitions and worldwide incidence and mortality. Epilepsia 49 Suppl 1: 8-12.

Hauser WA and Kurland LT (1975).The epidemiology of epilepsy in Rochester, Minnesota, 1935 through 1967. Epilepsia 16: 1-66.

Hauser WA, Rich SS, Annegers JF et al (1990). Seizure recurrence after a 1st unprovoked seizure: an extended follow-up. Neurology 40: 1163-1170.

Hauser WA, Rich SS, Lee JR et al (1998). Risk of recurrent seizures after two unprovoked seizures. N Engl J Med 338: 429-434.

Haynes CL, Cook GA and Jones MA (2007). Legal and ethical considerations in processing patient-identifiable data without patient consent: lessons learnt from developing a disease register. J Med Ethics 33: 302-307.

Heaney DC, MacDonald BK, Everitt A et al (2002). Socioeconomic variation in incidence of epilepsy: prospective community based study in south east England. BMJ 325: 1013-1016. 
Hennessy MJ, Langan Y, Elwes RD et al (1999). A study of mortality after temporal lobe epilepsy surgery. Neurology 53: 1276-1283.

Henriksson PB, Juul-Jensen P and Lund M (1967). The mortality of epileptics. Epilepsy and insurance. Social studies on epilepsy, No 5. Ed. International Bureau for Epilepsy, London, pp. 5-12.

Herman ST (2002). Epilepsy after brain insult: targeting epileptogenesis. Neurology 59: S21-S26.

Hesdorffer DC, Benn EKT, Cascino GD et al (2009). Is a first acute symptomatic seizure epilepsy? Mortality and risk for recurrent seizure. Epilepsia 50: 1102-1108.

Hesdorffer DC and D'Amelio M (2005). Mortality in the first 30 days following incident acute symptomatic seizures. Epilepsia 46 Supp1 11: 43-45.

Hesdorffer DC, Hauser WA, Annegers JF et al (1996). Dementia and adult-onset unprovoked seizures. Neurology 46: 727-730.

Hesdorffer DC, Logroscino G, Cascino G et al (1998a). Incidence of status epilepticus in Rochester, Minnesota, 1965-1984. Neurology 50: 735-741.

Hesdorffer DC, Logroscino G, Cascino G et al (1998b). Risk of unprovoked seizure after acute symptomatic seizure: effect of status epilepticus. Ann Neurol 44: 908-912.

Hesdorffer DC and Kanner AM (2009). The FDA alert on suicidality and antiepileptic drugs: Fire or false alarm? Epilepsia 50: 978-986.

Hesdorffer DC, Tian H, Anand K et al (2005). Socioeconomic status is a risk factor for epilepsy in Icelandic adults but not in children. Epilepsia 46: 1297-1303. 
Higgins J (2003). The Patient Information Advisory Group and the use of patientidentifiable data. J Health Serv Res Policy 8 Suppl 1: S1:8-11.

Holtkamp M, Othman J, Buchheim K et al (2005). Predictors and prognosis of refractory status epilepticus treated in a neurological intensive care unit. J Neurol Neurosurg Psychiatry 76: 534-539.

Hopkins A, Garman A and Clarke C (1988). The first seizure in adult life. Value of clinical features, electroencephalography, and computerised tomographic scanning in prediction of seizure recurrence. Lancet 1: 721-726.

Hopkins A and Scambler G (1977). How doctors deal with epilepsy. Lancet 1: 183-186.

Huang M, Hong Z, Zeng J et al (2002). The prevalence of epilepsy in rural Jinshan in Shanghai. Zhonghua Yi Xue Za Zhi 23: 345-346.

Hui AC, Joynt GM, Li H et al (2003). Status epilepticus in Hong Kong Chinese: aetiology, outcome and predictors of death and morbidity. Seizure 12: 478-482.

Huttenlocher PR and Hapke RJ (1990). A follow-up study of intractable seizures in childhood. Ann Neurol 28: 699-705.

Idro R, Gwer S, Kahindi M et al (2008). The incidence, aetiology and outcome of acute seizures in children admitted to a rural Kenyan district hospital. BMC Pediatr 8:5.

Irani SR, Bera K, Waters P et al (2010). N-methyl-D-aspartate antibody encephalitis: temporal progression of clinical and paraclinical observations in a predominantly nonparaneoplastic disorder of both sexes. Brain 133: 1655-1667.

Irani SR, Michell AW, Lang B et al (2011). Faciobrachial Dystonic Seizures Precede Lgil Antibody Limbic Encephalitis. Ann Neurol 69: 892-900. 
Jackson C, Crossland L, Dennis M et al (2008). Assessing the impact of the requirement for explicit consent in a hospital-based stroke study. QJM 101: 281-289.

Jaitly R, Sgro JA, Towne AR et al (1997). Prognostic value of EEG monitoring after status epilepticus: a prospective adult study. J Clin Neurophysiol 14: 326-334.

Jallon P (2002). Epilepsy and epileptic disorders, an epidemiological marker? Contribution of descriptive epidemiology. Epileptic Disord 4: 1-13.

Jallon P, Goumaz M, Haenggeli C et al (1997). Incidence of first epileptic seizures in the canton of Geneva, Switzerland. Epilepsia 38: 547-552.

Jallon P, Loiseau P and Loiseau J (2001). Newly diagnosed unprovoked epileptic seizures: presentation at diagnosis in CAROLE study. Coordination Active du Reseau Observatoire Longitudinal de 1' Epilepsie. Epilepsia 42: 464-475.

Jallon P, Smadja D, Cabre P et al (1999). EPIMART: prospective incidence study of epileptic seizures in newly referred patients in a French Carribean island (Martinique). Epilepsia 40: 1103-1109.

Juul-Jensen P and Foldspang A (1983). Natural history of epileptic seizures. Epilepsia 24: 297-312.

Kaiser C, Asaba G, Leichsenring M et al (1998). High incidence of epilepsy related to onchocerciasis in West Uganda. Epilepsy Res 30: 247-251.

Kalita J, Misra UK and Patel R (2006). Initial EEG in status epilepticus is helpful in predicting seizure recurrence. Electromyogr Clin Neurophysiol 46: 139-144.

Kang DC, Lee YM, Lee J et al (2005). Prognostic factors of status epilepticus in children. Yonsei Med J 46: 27-33. 
Kapur J and MacDonald RL (1997). Rapid seizure-induced reduction of benzodiazepine and $\mathrm{Zn} 2+$ sensitivity of hippocampal dentate granule cell GABA A receptors. J

Neurosci 17: 7532-7540.

Karasalihoglu S, Oner N, Celtik,C et al (2003).Risk factors of status epilepticus in children. Pediatr Int 45: 429-434.

Kelvin EA, Hesdorffer DC, Bagiella E et al (2007). Prevalence of self-reported epilepsy in a multiracial and multiethnic community in New York City. Epilepsy Res 77: 141150.

Kho LK, Lawn ND, Dunne JW et al (2006). First seizure presentation: do multiple seizures within 24 hours predict recurrence? Neurology 67: 1047-1049.

Kim LG, Johnson TL, Marson AG et al (2006). Prediction of risk of seizure recurrence after a single seizure and early epilepsy: further results from the MESS trial. Lancet Neurol 5: 317-322.

Kirby S and Sadler RM (1995). Injury and death as a result of seizures. Epilepsia 36: $25-28$.

Klenerman P, Sander JW and Shorvon SD (1993). Mortality in patients with epilepsy: a study of patients in long term residential care. J Neurol Neurosurg Psychiatry 56: 149152.

Knake S, Rochon J, Fleischer S et al (2006). Status epilepticus after stroke is associated with increased long-term case fatality. Epilepsia 47: 2020-2026.

Knake S, Rosenow F, Vescovi M et al; Status Epilepticus Study Group Hessen (SESGH) (2001). Incidence of status epilepticus in adults in Germany: a prospective, population-based study. Epilepsia 42: 714-718. 
Kotsopoulos IA, van MT, Kessels FG et al (2002). Systematic review and meta-analysis of incidence studies of epilepsy and unprovoked seizures. Epilepsia 43: 1402-1409.

Koubeissi M and Alshekhlee A (2007). In-hospital mortality of generalized convulsive status epilepticus: a large US sample. Neurology 69: 886-893.

Krumholz A, Stern BJ and Weiss HD (1988). Outcome from coma after cardiopulmonary resuscitation: relation to seizures and myoclonus. Neurology 38: 401405.

Kurtz Z, Tookey P and Ross E (1998). Epilepsy in young people: 23 year follow up of the British national child development study. BMJ 316: 339-342.

Kwan P, Arzimanoglou A, Berg AT et al (2010). Definition of drug resistant epilepsy: consensus proposal by the ad hoc Task Force of the ILAE Commission on Therapeutic Strategies. Epilepsia; 51: 1069-1077.

Kwan P and Brodie MJ (2000). Early identification of refractory epilepsy. N Engl J Med 342: 314-319.

Kwan P and Brodie MJ (2002). Refractory epilepsy: a progressive, intractable but preventable condition? Seizure 11: 77-84.

Kwan P and Sander JW (2004). The natural history of epilepsy: an epidemiological view. J Neurol Neurosurg Psychiatry 75: 1376-1381.

Kwong KL, Chang K and Lam SY (2004). Features predicting adverse outcomes of status epilepticus in childhood. Hong Kong Med J 10: 156-159.

Labovitz DL, Hauser WA and Sacco RL (2001). Prevalence and predictors of early seizure and status epilepticus after first stroke. Neurology 57: 200-206. 
Lacroix J, Deal C, Gauthier M et al (1994). Admissions to a pediatric intensive care unit for status epilepticus: a 10-year experience. Crit Care Med 22: 827-832.

Lambrechtsen FA and Buchhalter JR (2008). Aborted and refractory status epilepticus in children: a comparative analysis. Epilepsia 49: 615-625.

Lamminpaa A, Pukkala E, Teppo L et al (2002).Cancer incidence among patients using antiepileptic drugs: a long-term follow-up of 28,000 patients. Eur J Clin Pharmacol 58: $137-141$.

Langan Y, Nashef L, Sander JW (2005). Case-control study of SUDEP. Neurology; 64: 1131-1133.

Lavados J, Germain L, Morales A et al (1992). A descriptive study of epilepsy in the district of El Salvador, Chile, 1984-1988. Acta Neurol Scand 85: 249-256.

Lawlor DA and Stone T (2001). Public health and data protection: an inevitable collision or potential for a meeting of minds? Int J Epidemiol 30: 1221-1225.

Lawn ND, Bamlet WR, Radhakrishnan K et al (2004). Injuries due to seizures in persons with epilepsy: a population-based study. Neurology 63: 1565-1570.

Leach JP, Lauder R, Nicolson A et al (2005). Epilepsy in the UK: misdiagnosis, mistreatment, and undertreatment? The Wrexham area epilepsy project. Seizure 14: 514-520.

Leone MA, Solari A and Beghi E (2006). Treatment of the first tonic-clonic seizure does not affect long-term remission of epilepsy. Neurology 67: 2227-2229.

Lesser RP (1996). Psychogenic seizures. Neurology 46: 1499-507. 
Lhatoo SD, Langan Y, MacDonald BK et al (1999). Sudden unexpected death: a rare event in a large community based prospective cohort with newly diagnosed epilepsy and high remission rates. J Neurol Neurosurg Psychiatry 66:692-693.

Lhatoo SD, Johnson AL, Goodridge DM et al (2001). Mortality in epilepsy in the first 11 to 14 years after diagnosis: multivariate analysis of a long-term, prospective, population-based cohort. Ann Neurol 49: 336-344.

Lhatoo SD and Sander JW (2001). The epidemiology of epilepsy and learning disability. Epilepsia 42 Suppl 1: 6-9.

Lhatoo SD and Sander JW (2005). Cause-specific mortality in epilepsy. Epilepsia 46 Suppl 11:36-39.

Lhatoo SD, Solomon JK, McEvoy AW et al (2003). A prospective study of the requirement for and the provision of epilepsy surgery in the United Kingdom. Epilepsia 44: 673-676.

Li SC, Schoenberg BS, Wang CC et al (1985). Epidemiology of epilepsy in urban areas of the People's Republic of China. Epilepsia 26: 391-394.

Lin JJ, Lin KL, Wang HS et al (2008). Analysis of status epilepticus related presumed encephalitis in children. Eur J Paediatr Neurol 12: 32-37.

Lindsten H, Nystrom L and Forsgren L (2000). Mortality risk in an adult cohort with a newly diagnosed unprovoked epileptic seizure: a population-based study. Epilepsia 41: 1469-1473. 
Lindsten H, Stenlund H and Forsgren L (2001a). Remission of seizures in a populationbased adult cohort with a newly diagnosed unprovoked epileptic seizure. Epilepsia 42: 1025-1030.

Lindsten H, Stenlund H and Forsgren L (2001b). Seizure recurrence in adults after a newly diagnosed unprovoked epileptic seizure. Acta Neurol Scand 104: 202-207.

Linehan C, Kerr MP, Walsh PN et al (2010). Examining the prevalence of epilepsy and delivery of epilepsy care in Ireland. Epilepsia 51: 845-852.

Logroscino G and Hesdorffer DC (2005). Methodologic issues in studies of mortality following epilepsy: measures, types of studies, sources of cases, cohort effects, and competing risks. Epilepsia 46 Suppl 11: 3-7.

Logroscino G, Hesdorffer DC, Cascino G et al (1997). Short-term mortality after a first episode of status epilepticus. Epilepsia 38: 1344-1349.

Logroscino G, Hesdorffer DC, Cascino GD et al (2002). Long-term mortality after a first episode of status epilepticus. Neurology 58: 537-541.

Logroscino G, Hesdorffer DC, Cascino G et al (2005). Mortality after a first episode of status epilepticus in the United States and Europe. Epilepsia 46 Suppl 11: 46-48.

Logroscino G, Hesdorffer DC, Cascino G et al (2008). Status epilepticus without an underlying cause and risk of death: a population-based study. Arch Neurol 65: 221-224.

Loiseau J, Loiseau P, Guyot M et al (1990). Survey of seizure disorders in the French southwest. I. Incidence of epileptic syndromes. Epilepsia 31: 391-396.

Loiseau J, Picot MC and Loiseau P (1999). Short-term mortality after a first epileptic seizure: a population-based study. Epilepsia 40: 1388-1392. 
Lossius MI, Hessen E, Mowinckel P et al (2008). Consequences of antiepileptic drug withdrawal: a randomized, double-blind study (Akershus Study). Epilepsia 49: 455-463.

Lossius MI, Stavem K and Gjerstad L (1999). Predictors for recurrence of epileptic seizures in a general epilepsy population. Seizure 8: 476-479.

Lowenstein DH (1999). Status epilepticus: an overview of the clinical problem. Epilepsia 40 Suppl 1: 3-8.

Lowenstein DH and Alldredge BK (1993). Status epilepticus at an urban public hospital in the 1980s. Neurology 43: 483-488.

Luciano AL and Shorvon SD (2007). Results of treatment changes in patients with apparently drug-resistant chronic epilepsy. Ann Neurol 62: 375-381.

Mac TL, Tran DS, Quet F et al (2007). Epidemiology, aetiology, and clinical management of epilepsy in Asia: a systematic review. Lancet Neurol 6: 533-543.

MacDonald BK, Cockerell OC, Sander JW et al (2000). The incidence and lifetime prevalence of neurological disorders in a prospective community-based study in the UK. Brain 123: 665-676.

MacDonald BK, Johnson AL, Goodridge DM et al (2000). Factors predicting prognosis of epilepsy after presentation with seizures. Ann Neurol 48: 833-841.

MacDonald BK, Johnson AL, Sander JW et al (1999). Febrile convulsions in 220 children--neurological sequelae at 12 years follow-up. Eur Neurol 41: 179-186.

Maegaki Y, Kurozawa Y, Hanaki K et al (2005). Risk factors for fatality and neurological sequelae after status epilepticus in children. Neuropediatrics 36: 186-192. 
Maharaj M, Henry D, Ali K et al (1992) Status epilepticus. Recent experience at the Port-of-Spain General Hospital, Trinidad. West Indian Med J 41: 19-22.

Malfroy M, Llewelyn CA, Johnson T et al (2004). Using patient-identifiable data for epidemiological research. Transfus Med 14: 275-279.

Malzberg,B., 1934. Mortality among patients with mental disease. State Hospital Press, Utica, NY.

Manford M, Hart YM, Sander JW et al (1992). The National General Practice Study of Epilepsy. The syndromic classification of the International League Against Epilepsy applied to epilepsy in a general population. Arch Neurol 49: 801-808.

Mani KS, Rangan G, Srinivas HV et al (1998). The Yelandur study: a community-based approach to epilepsy in rural South India--epidemiological aspects. Seizure 7: 281-288.

Marson A, Jacoby A, Johnson A et al (2005). Immediate versus deferred antiepileptic drug treatment for early epilepsy and single seizures: a randomised controlled trial. Lancet 365: 2007-2013.

Massey EW and Schoenberg BS (1985). Mortality from epilepsy. International patterns and changes over time. Neuroepidemiology 4: 65-70.

Mayer SA, Claassen J, Lokin J et al (2002). Refractory status epilepticus: frequency, risk factors, and impact on outcome. Arch Neurol 59: 205-210.

Maytal J, Shinnar S, Moshe SL et al (1989). Low morbidity and mortality of status epilepticus in children. Pediatrics 83: 323-331.

Mbika Cardorelle A, Okoko AR, Yoka-Mamate AD et al (2005). L'état de mal convulsif en réanimation pédiatrique à Brazzaville. Arch Pédiatr 12(9):1400-1401. 
Mbodj I, Ndiaye M, Sene F et al (2000) Prise en charge de l'état de mal épileptique dans les conditions de pays en développement. Neurophysiol Clin 30: 165-169.

Mbuba CK, Ngugi AK, Newton CR et al (2008). The epilepsy treatment gap in developing countries: a systematic review of the magnitude, causes, and intervention strategies. Epilepsia 49: 1491-1503.

McDermott S, Moran R, Platt T et al (2005). Prevalence of epilepsy in adults with mental retardation and related disabilities in primary care. Am J Ment Retard 110: 4856.

Medina MT, Duron RM, Martinez L et al (2005). Prevalence, incidence, and etiology of epilepsies in rural Honduras: the Salama Study. Epilepsia 46: 124-131.

Meinardi H, Scott RA, Reis R et al (2001). The treatment gap in epilepsy: the current situation and ways forward. Epilepsia 42: 136-149.

Menon B and Shorvon SD (2009). Ischaemic stroke in adults and epilepsy. Epilepsy Res 87: 1-11.

Metcalfe C, Martin RM, Noble S et al (2008). Low risk research using routinely collected identifiable health information without informed consent: encounters with the Patient Information Advisory Group. J Med Ethics 34: 37-40.

Mignard C, Tchalla E, Marin B et al (2009).Incidence of newly diagnosed epileptic seizures in a French South Indian Ocean Island, La Réunion (EPIREUN). Epilepsia 50: $2207-2212$.

Mohanraj R, Norrie J, Stephen LJ et al (2006). Mortality in adults with newly diagnosed and chronic epilepsy: a retrospective comparative study. Lancet Neurol 5: 481-487. 
Montano SM, Villaran MV, Ylquimiche L et al (2005).Neurocysticercosis: association between seizures, serology, and brain CT in rural Peru. Neurology 65: 229-33.

Morgan CL, Ahmed Z and Kerr MP (2000). Social deprivation and prevalence of epilepsy and associated health usage. J Neurol Neurosurg Psychiatry 69: 13-17.

Morton B, Richardson A and Duncan S (2006). Sudden unexpected death in epilepsy (SUDEP): don't ask, don’t tell? J Neurol Neurosurg Psychiatry 77: 199-202.

MRC AED Withdrawal Study Group (1991). Randomised study of antiepilpetic drug withdrawal in patients in remission. Lancet 337: 1175-1180.

Mung'ala-Odera V, White S, Meehan R et al (2008).Prevalence, incidence and risk factors of epilepsy in older children in rural Kenya. Seizure 17: 396-404.

Munson JF (1910). Death in Epilepsy. Med Record 77: 58-62.

Muntner P, Garrett E, Klag MJ et al (2002). Trends in stroke prevalence between 1973 and 1991 in the US population 25 to 74 years of age. Stroke 33: 1209-1213.

Murthy JM, Jayalaxmi SS and Kanikannan MA (2007). Convulsive status epilepticus: clinical profile in a developing country. Epilepsia 48: 2217-2223.

Najjar S, Bernbaum M, Lai G et al (2008). Immunology and epilepsy. Rev Neurol Dis 5: 109-116.

Nashef L, Fish DR, Garner S et al (1995). Sudden death in epilepsy: a study of incidence in a young cohort with epilepsy and learning difficulty. Epilepsia 36: 11871194. 
Ndoye NF, Sow AD, Diop AG et al (2005). Prevalence of epilepsy its treatment gap and knowledge, attitude and practice of its population in sub-urban Senegal an ILAE/IBE/WHO study. Seizure 14: 106-111.

Nei M, Lee JM, Shanker VL et al (1999).The EEG and prognosis in status epilepticus. Epilepsia 40: 157-163.

Neligan A, Bell GS, Johnson AL et al (2010b). Long-Term Prognosis in Patients with Epilepsy. Epilepsia 51 Suppl 4: 69-70.

Neligan A, Bell GS, Johnson AL et al (2011a). The long-term risk of premature mortality in people with epilepsy. Brain 134: 388-395.

Neligan A, Bell GS, Sander JW et al (2011b). How refractory is refractory epilepsy? Patterns of relapse and remission in people with refractory epilepsy. Epilepsy Res $96: 225-230$.

Neligan A, Bell GS, Shorvon SD et al (2010a). Temporal trends in the mortality of people with epilepsy: A review. Epilepsia 51: 2241-2246.

Neligan A, Oomer S, Ziso B et al (2011c). A case of prolonged status epilepticus with a good outcome: The importance of aetiology in determining prognosis. Epilepsia 52 (Suppl 8): 83.

Neligan A and Shorvon SD (2011). Prognostic factors, morbidity and mortality in tonicclonic status epilepticus: a review. Epilepsy Res 93: 1-10.

Neligan A and Shorvon SD (2010). Frequency and prognosis of convulsive status epilepticus of different causes: a systematic review. Arch Neurol 67: 931-940. 
Neligan A and Shorvon SD (2008). Refractory versus non-refractory status epilepticus: frequency, differentiating clinical features, and outcome. Kahane,P., Berg,A.T., Loscher,W., Perucca,E. (Eds.) Drug-Resistant Epilepsies. John Libbey EUROTEXT, pp. 29-46.

Nelson KB and Ellenberg JH (1978). Prognosis in children with febrile seizures. Pediatrics 61: 720-727.

Ngoungou EB, Dulac O, Poudiougou B et al (2006a). Epilepsy as a consequence of cerebral malaria in area in which malaria is endemic in Mali, West Africa. Epilepsia 47: 873-879.

Ngoungou EB, Koko J, Druet-Cabanac M et al (2006b). Cerebral malaria and sequelar epilepsy: first matched case-control study in Gabon. Epilepsia 47: 2147-2153.

Ngugi AK, Bottomley C, Kleinschmidt I et al (2010). Estimation of the burden of active and life-time epilepsy: a meta-analytic approach. Epilepsia 51: 883-890.

Nicoletti A, Bartoloni A, Reggio A et al (2002).Epilepsy, cysticercosis, and toxocariasis: a population-based case-control study in rural Bolivia. Neurology 58: $1256-1261$.

Nicoletti A, Reggio A, Bartoloni A et al (1999). Prevalence of epilepsy in rural Bolivia: a door-to-door survey. Neurology 53: 2064-2069.

Nicoletti A, Sofia V, Mantella A et al (2008).Epilepsy and toxocariasis: a case-control study in Italy. Epilepsia 49: 594-599.

Nicoletti,A, Sofia,V., Vitale,G.et al (2009). Natural history and mortality of chronic epilepsy in an untreated population of rural Bolivia: a follow-up after 10 years. Epilepsia 50: 2199-2206. 
Nilsson L, Tomson T, Farahmand BY et al (1997). Cause-specific mortality in epilepsy: a cohort study of more than 9,000 patients once hospitalized for epilepsy. Epilepsia 38: 1062-1068.

Nkwi PN and Ndonko FT (1989). The epileptic among the Bamileke of Maham in the Nde Division, West Province of Cameroon. Cult Med Psychiatry 13: 437-448.

O'Callaghan FJ, Osmond C and Martyn CN (2000). Trends in epilepsy mortality in England and Wales and the United States, 1950-1994. Am J Epidemiol 151: 182-189.

O'Donoghue MF and Sander JW (1997). A historical perspective on the mortality associated with chronic epilepsy. Acta Neurol Scand 96: 138-141.

Ogunniyi A, Ogunniyi JO, Bademosi O et al (1992) Aetiology of status epilepticus in Ibadan: a neuropathologic study. West Afr J Med 11: 263-267.

Oka E, Ohtsuka Y, Yoshinaga H et al (2006). Prevalence of childhood epilepsy and distribution of epileptic syndromes: a population-based survey in Okayama, Japan. Epilepsia 47: 626-630.

Olafsson E, Gudmundsson G and Hauser WA (2000). Risk of epilepsy in long-term survivors of surgery for aneurysmal subarachnoid hemorrhage: a population-based study in Iceland. Epilepsia 41: 1201-1205.

Olafsson E and Hauser WA (1999). Prevalence of epilepsy in rural Iceland: a population-based study. Epilepsia 40: 1529-1534.

Olafsson E, Hauser WA and Gudmundsson G (1998). Long-term survival of people with unprovoked seizures: a population-based study. Epilepsia 39: 89-92. 
Olafsson E, Hauser WA, Ludvigsson P et al (1996). Incidence of epilepsy in rural Iceland: a population-based study. Epilepsia 37: 951-955.

Olafsson E, Ludvigsson P, Gudmundsson G et al (2005). Incidence of unprovoked seizures and epilepsy in Iceland and assessment of the epilepsy syndrome classification: a prospective study. Lancet Neurol 4: 627-634.

Oka E, Yamatogi Y, Ohtsuka Y et al (1989). Clinical course and prognosis of childhood epilepsy. Acta Paediatr Jpn 31: 259-266.

Okuma T and Kumashiro H (1981). Natural history and prognosis of epilepsy: report of a multi-institutional study in Japan. The group for the study of prognosis of epilepsy in Japan. Epilepsia 22: 35-53.

Olsen JH, Boice JD Jr, Jensen JP et al (1989). Cancer among epileptic patients exposed to anticonvulsant drugs. J Natl Cancer Inst 81: 803-808.

Oun A, Haldre S and Magi M (2003). Incidence of adult epilepsy in Estonia. Acta Neurol Scand 108: 245-251.

Pitkänen A (2010). Therapeutic approaches to epileptogenesis--hope on the horizon. Epilepsia 51 Suppl 3:2-17.

Pitkänen A and Lukasiuk K (2011). Mechanisms of epileptogenesis and potential treatment targets. Lancet Neurol 10:173-186.

Pitkänen A, Kharatishvili I, Karhunen H et al (2007). Epileptogenesis in experimental models. Epilepsia 48 Suppl 2:13-20. 
Placencia M, Sander JW, Roman M et al (1994). The characteristics of epilepsy in a largely untreated population in rural Ecuador. J Neurol Neurosurg Psychiatry 57: 320325.

Placencia M, Shorvon SD, Paredes V et al (1992). Epileptic seizures in an Andean region of Ecuador. Incidence and prevalence and regional variation. Brain 115 ( Pt 3): 771-782.

Preux PM and Druet-Cabanac M (2005). Epidemiology and aetiology of epilepsy in sub-Saharan Africa. Lancet Neurol 4: 21-31.

Pritchard C, Baldwin D and Mayers A (2004). Changing patterns of adult (45-74 years) neurological deaths in the major Western world countries 1979-1997. Public Health 118: $268-283$.

Proceedings of the Innsbruck Colloquium on Status Epilepticus. Innsbruck, Austria. April 2-5, 2009. Epilepsia 50 Suppl 12: 1-80.

Provisional Committee on Quality Improvemnets SoFS (1996). Practice Parameter: The Neurodiagnostic Evaluation of the Child With a First Simple Febrile Seizure. Pediatrics 97: 769-772.

Rafnsson V, Olafsson E, Hauser WA et al (2001). Cause-specific mortality in adults with unprovoked seizures. A population-based incidence cohort study. Neuroepidemiology 20: 232-236.

Rajshekhar V, Raghava MV, Prabhakaran V et al (2006). Active epilepsy as an index of burden of neurocysticercosis in Vellore district, India. Neurology 67: 2135-2139. 
Rao SC, Dove G, Cascino GD et al (2009). Recurrent seizures in patients with dementia: frequency, seizure types, and treatment outcome. Epilepsy Behav 14: 118120.

Raspall-Chaure M, Chin RF, Neville BG et al (2006). Outcome of paediatric convulsive status epilepticus: a systematic review. Lancet Neurol 5: 769-779.

Rocca WA, Savettieri G, Anderson DW et al (2001). Door-to-door prevalence survey of epilepsy in three Sicilian municipalities. Neuroepidemiology 20: 237-241.

Rodin,E.A., 1968. The prognosis of patients with epilepsy. Charles C Thomas, Springfield, IL.

Rossetti AO, Hurwitz S, Logroscino G. et al (2006). Prognosis of status epilepticus: role of aetiology, age, and consciousness impairment at presentation. J Neurol Neurosurg Psychiatry 77: 611-615.

Rossetti AO, Logroscino G and Bromfield EB (2005). Refractory status epilepticus: effect of treatment aggressiveness on prognosis. Arch Neurol 62: 1698-1702.

Rossetti AO, Logroscino G, Liaudet L et al (2007). Status epilepticus: an independent outcome predictor after cerebral anoxia. Neurology 69: 255-260.

Rossetti AO, Reichhart MD, Schaller MD et al (2004). Propofol treatment of refractory status epilepticus: a study of 31 episodes. Epilepsia 45: 757-763.

Rugg-Gunn FJ, Harrison NA and Duncan JS (2001). Evaluation of the accuracy of seizure descriptions by the relatives of patients with epilepsy. Epilepsy Res 43: 193199. 
Rumbach L, Sablot D, Berger E et al (2000). Status epilepticus in stroke: report on a hospital-based stroke cohort. Neurology 54: 350-354.

Rwiza HT, Kilonzo GP, Haule J et al (1992). Prevalence and incidence of epilepsy in Ulanga, a rural Tanzanian district: a community-based study. Epilepsia 33: 1051-1056.

Sadarangani M, Seaton,C, Scott JA et al (2008). Incidence and outcome of convulsive status epilepticus in Kenyan children: a cohort study. Lancet Neurol 7: 145-150.

Sagduyu A, Tarlaci S and Sirin H (1998).Generalized tonic-clonic status epilepticus: causes, treatment, complications and predictors of case fatality. J Neurol 245: 640-646.

Sahin M, Menache CC, Holmes GL et al (2001).Outcome of severe refractory status epilepticus in children. Epilepsia 42: 1461-1467.

Saito Y, Maegaki Y, Okamoto R et al (2007). Acute encephalitis with refractory, repetitive partial seizures: case reports of this unusual post-encephalitic epilepsy. Brain Dev 29: 147-156.

Sander JW (1993). Some aspects of prognosis in the epilepsies: a review. Epilepsia 34: $1007-1016$.

Sander JW (2003). The epidemiology of epilepsy revisited. Curr Opin Neurol 16: 165170.

Sander JW, Hart YM, Johnson AL et al (1990). National General Practice Study of Epilepsy: newly diagnosed epileptic seizures in a general population. Lancet 336: 1267 1271. 
Sander JW and Perucca E (2003). Epilepsy and comorbidity: infections and antimicrobials usage in relation to epilepsy management. Acta Neurol Scand Suppl 180: 16-22.

Sander JW and Shorvon SD (1987). Remission periods in epilepsy and their relation to long-term prognosis. In: Wolf P, Dam M, Janz D, editors. Advances in Epileptology. New York: Raven Press; 353-356.

Sander JW and Shorvon SD (1987). Incidence and prevalence studies in epilepsy and their methodological problems: a review. J Neurol Neurosurg Psychiatry 50: 829-839.

Sander JW and Shorvon SD (1996). Epidemiology of the epilepsies. J Neurol Neurosurg Psychiatry 61: 433-443.

Sawhney IM, Singh A, Kaur P et al (1999). A case control study and one year follow-up of registered epilepsy cases in a resettlement colony of North India, a developing tropical country. J Neurol Sci. 165: 31-35.

Schiller Y (2009). Seizure relapse and development of drug resistance following longterm seizure remission. Arch Neurol; 66: 1233-1239.

Schiller Y and Najjar Y (2008). Quantifying the response to antiepileptic drugs: effect of past treatment history. Neurology; 70: 54-65.

Schmidt D and Loscher W (2005). Uncontrolled epilepsy following discontinuation of antiepileptic drugs in seizure-free patients: a review of current clinical experience. Acta Neurol Scand 111: 291-300.

Schmidt D and Stavem K (2009). Long-term seizure outcome of surgery versus no surgery for drug-resistant partial epilepsy: A review of controlled studies. Epilepsia 50: 1301-1309. 
Scholtes FB, Renier WO and Meinardi H (1994). Generalized convulsive status

epilepticus: causes, therapy, and outcome in 346 patients. Epilepsia 35: 1104-1112.

Semah F, Picot MC, Adam C et al (1998). Is the underlying cause of epilepsy a major prognostic factor for recurrence? Neurology 51: 1256-1262.

Shackleton DP, Westendorp RG, Kasteleijn-Nolst Trenite DG et al (2002). Survival of patients with epilepsy: an estimate of the mortality risk. Epilepsia 43: 445-450.

Shackleton DP, Westendorp RG, Trenite DG et al (1999). Mortality in patients with epilepsy: 40 years of follow up in a Dutch cohort study. J Neurol Neurosurg Psychiatry 66: $636-640$.

Shafer SQ, Hauser WA, Annegers JF et al (1988). EEG and other early predictors of epilepsy remission: a community study. Epilepsia 29: 590-600.

Shamansky SL and Glaser GH (1979). Socioeconomic characteristics of childhood seizure disorders in the New Haven area: an epidemiologic study. Epilepsia 20: 457474.

Shinnar S, Berg AT, Moshe SL et al (1996).The risk of seizure recurrence after a first unprovoked afebrile seizure in childhood: an extended follow-up. Pediatrics 98: 216225.

Shinnar S, Berg AT, O'Dell C et al (2000). Predictors of multiple seizures in a cohort of children prospectively followed from the time of their first unprovoked seizure. Ann Neurol 48: 140-147.

Shinnar S, Maytal J, Krasnoff L et al (1992).Recurrent status epilepticus in children. Ann Neurol 31: 598-604. 
Shinnar S, O'Dell C and Berg AT (1999). Distribution of epilepsy syndromes in a cohort of children prospectively monitored from the time of their first unprovoked seizure. Epilepsia 40: 1378-1383.

Shinnar S, O'Dell C and Berg AT (2005). Mortality following a first unprovoked seizure in children: a prospective study. Neurology 64: 880-882.

Shinnar S, Pellock JM, Berg AT et al (2001). Short-term outcomes of children with febrile status epilepticus. Epilepsia 42: 47-53.

Shorvon SD (1984). The temporal aspects of prognosis in epilepsy J Neurol Neurosurg Psychiatry; 47:1157-1165

Shorvon,SD (1994). Status Epilepticus: its clinical features and treatment in children and adults. Cambridge University Press, Cambridge.

Shorvon SD and Ferlisi M (2011). The treatment of super-refractory status epilepticus: a critical review of available therapies and a clinical treatment protocol. Brain 134: 28022818.

Shorvon SD and Sander JW (1986). Temporal patterns of remission and relapse in patients with epilepsy. Schmidt D, Morceli P. (Eds.) Intractable Epilepsy: experimental and clinical aspects. Raven Press, New York, pp. 13-23

Shorvon SD, Trinka E and Walker MC (2007). The proceedings of the First London Colloquium on Status Epilepticus--University College London, April 12-15, 2007. Introduction. Epilepsia 48 Suppl 8: 1-3.

Sidenvall R, Forsgren L, Blomquist HK et al (1993). A community-based prospective incidence study of epileptic seizures in children. Acta Paediatr 82: 60-65. 
Sidenvall R, Forsgren L and Heijbel J (1996). Prevalence and characteristics of epilepsy in children in northern Sweden. Seizure 5: 139-146.

SillanpaaM (1993).Remission of seizures and predictors of intractability in long-term follow-up. Epilepsia 34:930-936.

Sillanpaa M (2000). Long-term outcome of epilepsy. Epileptic Disord 2: 79-88.

Sillanpaa M, Camfield P, Camfield C et al (2008). Incidence of febrile seizures in Finland: prospective population-based study. Pediatr Neurol 38: 391-394.

Sillanpaa M, Jalava M, Kaleva O et al (1998). Long-term prognosis of seizures with onset in childhood. N Engl J Med 338: 1715-1722.

Sillanpaa M, Kalviainen R, Klaukka T et al (2006). Temporal changes in the incidence of epilepsy in Finland: nationwide study. Epilepsy Res 71: 206-215.

Sillanpaa M and Schmidt D (2006a). Natural history of treated childhood-onset epilepsy: prospective, long-term population-based study. Brain 129: 617-624.

Sillanpaa M and Schmidt D (2006b). Prognosis of seizure recurrence after stopping antiepileptic drugs in seizure-free patients: A long-term population-based study of childhood-onset epilepsy. Epilepsy Behav 8: 713-719.

Sillanpaa M and Schmidt D (2008). Seizure clustering during drug treatment affects seizure outcome and mortality of childhood-onset epilepsy. Brain 131: 938-944.

Sillanpaa M and Schmidt D (2009). Early seizure frequency and aetiology predict longterm medical outcome in childhood-onset epilepsy. Brain 132; 989-998

Sillanpaa M and Shinnar S (2002). Status epilepticus in a population-based cohort with childhood-onset epilepsy in Finland. Ann Neurol 52: 303-310. 
Sillanpaa M and Shinnar S (2010). Long-Term Mortality in Childhood-Onset

Epilepsy.N Engl J Med; 363: 2522-2529

Singh G, Fletcher O, Bell GS et al (2009). Cancer mortality amongst people with epilepsy: a study of two cohorts with severe and presumed milder epilepsy. Epilepsy Res 83: 190-197.

Smith D, Defalla BA and Chadwick DW (1999). The misdiagnosis of epilepsy and the management of refractory epilepsy in a specialist clinic. QJM 92: 15-23.

So EL, Annegers JF, Hauser WA et al (1996).Population-based study of seizure disorders after cerebral infarction. Neurology 46: 350-355.

Sokic DV, Jankovic SM, Vojvodic NM et al (2009). Aetiology of a short-term mortality in the group of 750 patients with 920 episodes of status epilepticus within a period of 10 years (1988-1997). Seizure 18: 215-219.

Sperling MR, Feldman H, Kinman J et al (1999). Seizure control and mortality in epilepsy. Ann Neurol 46: 45-50.

Sperling MR, Harris A, Nei M et al (2005). Mortality after epilepsy surgery. Epilepsia 46 Suppl 11: 49-53.

Sridharan R and Murthy BN (1999). Prevalence and pattern of epilepsy in India. Epilepsia 40: 631-636.

Stephen LJ, Kwan P and Brodie MJ (2001). Does the cause of localisation-related epilepsy influence the response to antiepileptic drug treatment? Epilepsia 42: 357-362.

Strauss DJ, Day SM, Shavelle RM et al (2003). Remote symptomatic epilepsy: does seizure severity increase mortality? Neurology 60: 395-399. 
Stroink H, Brouwer OF, Arts WF et al (1998). The first unprovoked, untreated seizure in childhood: a hospital based study of the accuracy of the diagnosis, rate of recurrence, and long term outcome after recurrence. Dutch study of epilepsy in childhood. J Neurol Neurosurg Psychiatry 64: 595-600.

Stroink H, Geerts AT, van Donselaar CA et al (2007). Status epilepticus in children with epilepsy: Dutch study of epilepsy in childhood. Epilepsia 48: 1708-1715.

Stroink H, van Donselaar CA, Geerts AT et al (2004). Interrater agreement of the diagnosis and classification of a first seizure in childhood. The Dutch Study of Epilepsy in Childhood. J Neurol Neurosurg Psychiatry 75: 241-245.

Szaflarski JP, Rackley AY, Kleindorfer DO et al (2008).Incidence of seizures in the acute phase of stroke: a population-based study. Epilepsia 49: 974-981.

Tabarki B, Yacoub M, Selmi H et al (2001). Infantile status epilepticus in Tunisia. Clinical, etiological and prognostic aspects. Seizure 10: 365-369.

Tan RYL, Neligan A and Shorvon SD (2010). The uncommon causes of status epilepticus: A Systemic Review. Epilepsy Res 91: 111-122.

Tekle-Haimanot R, Forsgren L and Ekstedt J (1997). Incidence of epilepsy in rural central Ethiopia. Epilepsia 38: 541-546.

Téllez-Zenteno JF, Matijevic S and Wiebe S (2005). Somatic comorbidity of epilepsy in the general population in Canada. Epilepsia 46: 1955-1962.

Theodore WH, Porter RJ, Albert P et al (1994). The secondarily generalized tonicclonic seizure: a videotape analysis. Neurology 44: 1403-1407. 
Theodore WH, Spencer SS, Wiebe S et al (2006). Epilepsy in North America: a report prepared under the auspices of the global campaign against epilepsy, the International Bureau for Epilepsy, the International League Against Epilepsy, and the World Health Organization. Epilepsia 47: 1700-1722.

Thömke F, Marx JJ, Sauer O et al (2005). Observations on comatose survivors of cardiopulmonary resuscitation with generalized myoclonus. BMC Neurol 5:14.

Tomson T (2000). Mortality in epilepsy. J Neurol 247:15-21.

Tomson T, Walczak T, Sillanpaa M et al (2005). Sudden unexpected death in epilepsy: a review of incidence and risk factors. Epilepsia 46 Suppl 11: 54-61.

Towne AR, Pellock JM, Ko D et al (1994).Determinants of mortality in status epilepticus. Epilepsia 35: 27-34.

Treiman DM, Meyers PD, Walton NY et al (1998). A comparison of four treatments for generalized convulsive status epilepticus. Veterans Affairs Status Epilepticus Cooperative Study Group. N Engl J Med 339: 792-798.

Tsuboi T (1984). Epidemiology of febrile and afebrile convulsions in children in Japan. Neurology 34: 175-181.

Tu JV, Willison DJ, Silver FL et al (2004). Impracticability of informed consent in the Registry of the Canadian Stroke Network. N Engl J Med 350: 1414-1421.

Tuan NA, Cuong 1Q, Allebeck P et al (2008). The prevalence of epilepsy in a rural district of Vietnam: a population-based study from the EPIBAVI project. Epilepsia 49: 1634-1637. 
UK Parliament Acts. European Data Protection Act 1998. Available at:

http://www.hmso.gov.uk/acts1998/19980029.htm. First Accessed $18^{\text {th }}$ October 2007.

Van der Berg BJ and Yerushalmy J (1969). Studies on convulsive disorders in young children. I. Incidence of febrile and nonfebrile convulsions by age and other factors.

Pediatr Res 3: 298-304.

Van Donselaar CA, Geerts AT, Meulstee J et al (1989). Reliability of the diagnosis of a first seizure. Neurology 39: 267-271.

Van Paesschen W, Porke K, Fannes K et al (2007). Cognitive deficits during status epilepticus and time course of recovery: a case report. Epilepsia 48: 1979-1983.

Velez A and Eslava-Cobos J (2006). Epilepsy in Colombia: epidemiologic profile and classification of epileptic seizures and syndromes. Epilepsia 47: 193-201.

Velioğlu SK, Ozmenoğlu M, Boz C et al (2001). Status epilepticus after stroke. Stroke 32: 1169-1172.

Verburgh ME, Bruijnzeels MA, van der Wouden JC et al (1992). Incidence of febrile seizures in The Netherlands. Neuroepidemiology 11: 169-172.

Verity CM and Golding J (1991). Risk of epilepsy after febrile convulsions: a national cohort study. BMJ 303: 1373-1376.

Verity CM, Ross EM and Golding J (1993). Outcome of childhood status epilepticus and lengthy febrile convulsions: findings of national cohort study. BMJ 307: 225-228.

Vespa PM, Nuwer MR, Nenov V et al (1999). Increased incidence and impact of nonconvulsive and convulsive seizures after traumatic brain injury as detected by continuous electroencephalographic monitoring. J Neurosurg 91: 750-760. 
Vestergaard M, Pedersen CB, Sidenius P et al (2007). The long-term risk of epilepsy after febrile seizures in susceptible subgroups. Am J Epidemiol 165: 911-918.

Vestergaard M, Pedersen MG, Ostergaard JR et al (2008). Death in children with febrile seizures: a population-based cohort study. Lancet 372: 457-463.

Vezzani A and Granata T (2005).Brain inflammation in epilepsy: experimental and clinical evidence. Epilepsia 46: 1724-1743.

Vignatelli L, Rinaldi R, Baldin E et al (2008). Impact of treatment on the short-term prognosis of status epilepticus in two population-based cohorts. J Neurol 255 : 197-204.

Vignatelli,L, Rinaldi R, Galeotti M et al (2005). Epidemiology of status epilepticus in a rural area of northern Italy: a 2-year population-based study. Eur J Neurol 12: 897-902.

Vignatelli L, Tonon C and D'Alessandro R (2003). Incidence and short-term prognosis of status epilepticus in adults in Bologna, Italy. Epilepsia 44: 964-968.

Vincent A, Bien CG, Irani SR et al (2011a). Autoantibodies associated with diseases of the CNS : new developments and future challenges. Lancet Neurol 10: 759-772.

Vincent A, Irani SR and Lang B (2011b). Potentially pathogenic autoantibodies associated with epilepsy and encephalitis in children and adults. Epilepsia 52 (Suppl 8): $8-11$.

Visudtibhan A, Limhirun J, Chiemchanya S et al (2006). Convulsive status epilepticus in Thai children at Ramathibodi Hospital. J Med Assoc Thai 89: 803-808.

Wakamoto H, Nagao H, Hayashi M et al (2000). Long-term medical, educational, and social prognoses of childhood-onset epilepsy: a population-based study in a rural district of Japan. Brain Dev 22: 246-255. 
Wang W, Wu J, Wang D et al (2002). Epidemiological survey on epilepsy among rural populations in five provinces in China. Zhonghua Yi Xue Za Zhi 82: 449-452.

Wang WZ, Wu JZ, Wang DS et al (2003). The prevalence and treatment gap in epilepsy in China: an ILAE/IBE/WHO study. Neurology 60: 1544-1545.

Waterhouse EJ, Garnett LK, Towne AR et al (1999). Prospective population-based study of intermittent and continuous convulsive status epilepticus in Richmond, Virginia. Epilepsia 40: 752-758.

Waterhouse EJ, Vaughan JK, Barnes TY et al (1998). Synergistic effect of status epilepticus and ischemic brain injury on mortality. Epilepsy Res 29: 175-183.

Watts AE (1992). The natural history of untreated epilepsy in a rural community in Africa. Epilepsia 33: 464-468.

White SJ, McLean AE and Howland C (1979). Anticonvulsant drugs and cancer. A cohort study in patients with severe epilepsy. Lancet 2: 458-461.

Wijdicks EF, Hijdra A, Young GB et al (2006). Practice parameter: prediction of outcome in comatose survivors after cardiopulmonary resuscitation (an evidence-based review): report of the Quality Standards Subcommittee of the American Academy of Neurology. Neurology 67: 203-210.

Wijdicks EF and Young GB (1994). Myoclonus status in comatose patients after cardiac arrest. Lancet 343:1642-1643.

Wright J, Pickard N, Whitfield A et al (2000). A population-based study of the prevalence, clinical characteristics and effect of ethnicity in epilepsy. Seizure 9: 309313. 
Wu YW, Shek DW, Garcia PA et al (2002). Incidence and mortality of generalized convulsive status epilepticus in California. Neurology 58: 1070-1076.

Yaffe K and Lowenstein DH (1993). Prognostic factors of pentobarbital therapy for refractory generalized status epilepticus. Neurology 43: 895-900.

Zarrelli MM, Beghi E, Rocca WA et al (1999). Incidence of epileptic syndromes in Rochester, Minnesota: 1980-1984. Epilepsia 40: 1708-1714.

Zhao Y, Zhang Q, Tsering T et al (2008). Prevalence of convulsive epilepsy and healthrelated quality of life of the population with convulsive epilepsy in rural areas of Tibet Autonomous Region in China: an initial survey. Epilepsy Behav 12: 373-381.

Zielinski, JJ. (1974a) Epidemiology and Medicosocial Problems of Epilepsy in Warsaw. Final Report on Research Programno. 19-P-58325-F-01. Warsaw, Psychoneurological Institute.

Zielinski JJ (1974b). Epilepsy and mortality rate and cause of death. Epilepsia 15: 191201.

Zoons E, Weisfelt M, de Gans J et al (2008). Seizures in adults with bacterial meningitis. Neurology 70: 2109-2115. 


\section{Appendix 1}

Version $0.3 / 17 / 10 / 2007$

National General Practice Study of Epilepsy and Epileptic Seizures (NGPSE)

- Form: 20 Year Follow UP -

This is a follow up about a person who was registered by their then General Practitioner with the National General Practice Study of Epilepsy (NGPSE). This study was originally set up in 1983-84 with people registered in the study by their GPs between 1984 and 1987.

PLEASE RETURN THE COMPLETED FORM TO:

Professor J. W.Sander, FREEPOST, National Society for Epilepsy, Chalfont St Peter, Bucks., SL9 0RJ.

1. General Practitioner 2. Patient

Date of last recorded seizure: (filled in by the research team)

Seizure type: (filled in by the research team)

Has this person had an epileptic seizure in the last five years?

$$
\text { Yes No }
$$

Is this person currently taking anti-epileptic medication?

Yes No


If off medication, when was the medication withdrawn (year) .............

Have they had any seizures since last follow-up (1997)? Please give details (approximate dates and frequency)

If seizures did occur, were these seizures similar to previous seizures or different? (Has the diagnosis changed?)

Please give details

Please give details of current anti-epileptic medications, indicating doses 
Since 1997, have other medications been tried? - if so, please give details (type of medication and dates)

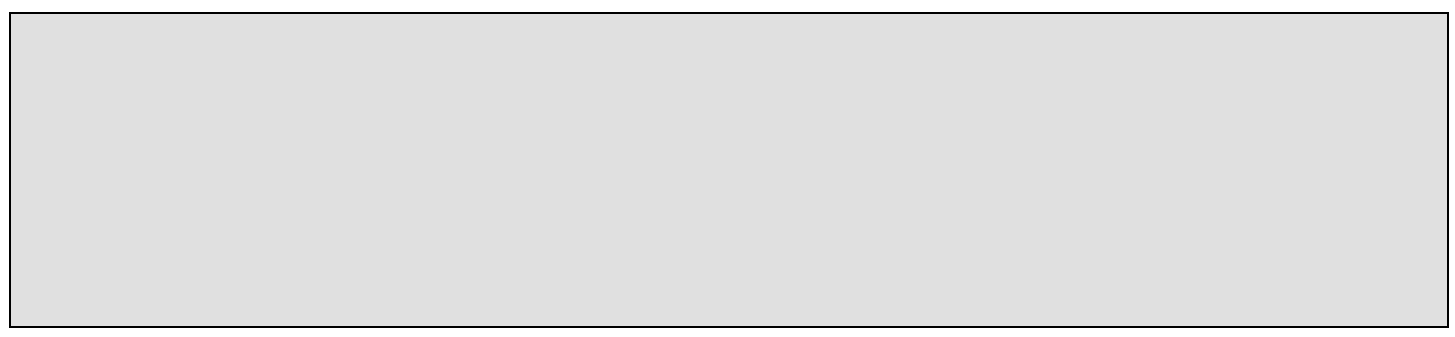

Is this person still attending a hospital outpatient clinic for seizures?

Yes No

Thank you for your cooperation 


\section{Appendix 2:}

Version 5: 16/01/2009

\section{National General Practice Study of Epilepsy and Epileptic Seizures (NGPSE)}

- Form: 20 Year Follow Up -

This is a follow up about a person who was registered by their then General Practitioner with the National General Practice Study of Epilepsy (NGPSE). This study was originally set up in 1983-84 with people registered in the study by their GPs between 1984 and 1987.

PLEASE RETURN THE COMPLETED FORM TO:

Professor J. W.Sander,

FREEPOST,

National Society for Epilepsy,

Chalfont St Peter,

Bucks., SL9 0RJ.

1. General Practitioner 2. Patient

Date of last recorded seizure: (completed by the research team)

Seizure type: (completed by the research team)

1) Has this person had an epileptic seizure since October 2004?

No

2) Has this person taken anti-epileptic medication since October 2004?

Yes

No

- If currently off medication, when was the medication withdrawn (year) 
If the answer to either of the above questions is YES we would like your assistance to seek and obtain the consent of the individual to obtain more information about them (see enclosed patient information sheet and consent form).

If the answer to both of the above two questions is NO, then we have approval under Section 251 of the NHS Act 2006 (formerly Section 60 of the Health and Social Care Act) to permit you to disclose the following information.

Has this person had any seizures since last follow-up (between 1997 \& 2004 inclusive)?

Please give details (approximate dates and frequency)

If seizures did occur, were these seizures similar to previous seizures or different? (Has the diagnosis changed?)

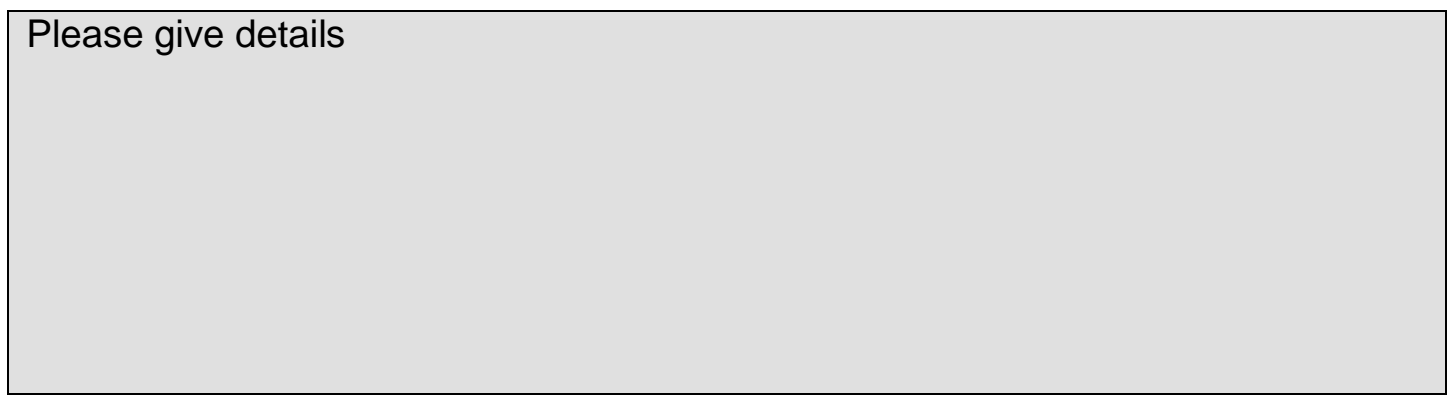


Please provide information of all anti-epileptic medications taken between 1997 and 2003

with dates.

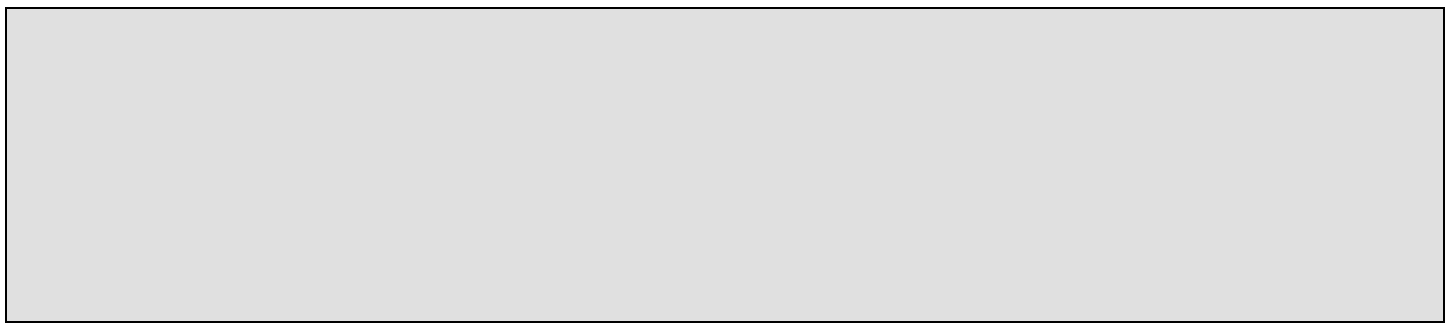

Please provide details of any known attendances at hospital outpatient clinics for seizures between 1997 and 2003

\section{Thank you for your cooperation}

